\title{
Composing security protocols: from confidentiality to privacy
}

\author{
Myrto Arapinis \\ School of Informatics \\ University of Edinburgh, UK
}

\author{
Vincent Cheval \\ LORIA, CNRS \\ Inria Nancy - Grand Est, France
}

\author{
Stéphanie Delaune \\ LSV, ENS Cachan \& CNRS \\ Inria Saclay Île-de-France, France
}

\begin{abstract}
Security protocols are used in many of our daily-life applications, and our privacy largely depends on their design. Formal verification techniques have proved their usefulness to analyse these protocols, but they become so complex that modular techniques have to be developed. We propose several results to safely compose security protocols. We consider arbitrary primitives modeled using an equational theory, and a rich process algebra close to the applied pi calculus.

Relying on these composition results, we are able to derive some security properties on a protocol from the security analysis performed on each sub-protocol individually. We consider parallel composition and the case of key-exchange protocols. Our results apply to deal with confidentiality but also privacy-type properties (e.g. anonymity, unlinkability) expressed using a notion of equivalence. We illustrate the usefulness of our composition results on protocols from the $3 \mathrm{G}$ phone application.
\end{abstract}

\section{INTRODUCTION}

Privacy means that one can control when, where, and how information about oneself is used and by whom, and it is actually an important issue in many modern applications. For instance, nowadays, it is possible to wave a building access card, a government-issued ID, or even a smartphone in front of a reader to go through a gate, or to pay for some purchase. Unfortunately, this technology also makes it possible for anyone to capture some of our personal information [1]. To secure the applications mentioned above and to protect our privacy, some specific cryptographic protocols are deployed. For instance, the $3 \mathrm{G}$ telecommunication application allows one to send SMS encrypted with a key that is established with the $A K A$ protocol [2]. The aim of this design is to provide some security guarantees: e.g. the SMS exchanged between phones should remain confidential from third parties.

Because security protocols are notoriously difficult to design and analyse, formal verification techniques are important. They have become mature and have known several successes. For instance, a flaw has been discovered in the Single-Sign-On protocol used by Google Apps [3], and several verification tools are available (e.g. ProVerif [4], the AVANTSSAR platform [5]). These tools perform well in practice, at least for standard security properties (e.g. secrecy, authentication). Regarding privacy properties, the techniques and tools are more recent. Most of the verification techniques are only able to analyse a bounded number of sessions and consider a quite restrictive class of protocols (e.g. fixed set of primitives and/or

ProVerif code are available at http://www.loria.fr/ chevalvi/other/phones/ no conditional branching [6], [7]). A slightly different approach consists in analysing a stronger notion of equivalence, namely diff-equivalence. In particular, ProVerif implements a semi-decision procedure for checking diff-equivalence [4].

Security protocols used in practice are more and more complex and it is difficult to analyse them entirely. For example, the UMTS standard [2] specifies tens of sub-protocols running in parallel in $3 \mathrm{G}$ phone systems. One may hope to verify each protocol in isolation. This is however unrealistic to expect that the whole application will be checked relying on a unique automatic tool. First, existing tools have their own specificities that prevent them to be used in some cases. Moreover, most of the techniques do not scale up well on large systems, and sometimes the ultimate solution is to rely on a manual proof. It is therefore important that the protocol under study is as small as possible.

Related work: There are many results studying the composition of security protocols in the symbolic model [8], [9], [10], as well as in the computational model [11], [12] in which the so-called UC (universal composability) framework has been first developed before to be adapted in the symbolic setting [13]. This result belongs to the first approach.

Most of the existing composition results concern trace-based security properties, and even secrecy (stated as a reachability property), e.g. [8], [9], [10], [14]. They are quite restricted in term of the class of protocols that can be composed, e.g. a fixed set of cryptographic primitives and/or no else branch. Lastly, they often only consider parallel composition. Some notable exceptions are the results presented in [15], [14], [10]. This paper is clearly inspired from the approach developed in [10].

Regarding privacy-type properties, very few composition results exist. In a previous work [16], we consider parallel composition only. More precisely, we identify sufficient conditions under which protocols can "safely" be executed in parallel as long as they have been proved secure in isolation. This composition theorem is quite general from the point of view of the cryptographic primitives. In particular, we consider arbitrary primitives that can be modelled by a set of equations, and protocols may share some standard primitives provided they are tagged differently. This is needed to ensure "disjointness" and to avoid damaging interactions between the sub-protocols. We choose to reuse this quite general setting in this work. 
Our contributions: Our main goal is to analyse privacytype properties in a modular way. These security properties are usually expressed as equivalences between processes. Roughly, two processes $P$ and $Q$ are equivalent $(P \approx Q)$ if, however they behave, the messages observed by the attacker are indistinguishable. Actually, it is well-known that if $P_{1} \approx P_{2}$ and $Q_{1} \approx Q_{2}$ then $P_{1}\left|P_{2} \approx Q_{1}\right| Q_{2}$. However, this parallel composition result works because processes that are composed are disjoint (e.g. they share no key). Moreover, in this work, we want to go beyond parallel composition. In particular, we want to capture the case where a protocol uses a sub-protocol to establish some keys.

To achieve our goal, we first enrich the applied pi calculus with an assignment construction. This will allow us to share some data (e.g. session keys) between sub-protocols. We present the calculus that is used in this paper to model security protocols in Section II, and we explain in Section III the difficulties that arise when composing security protocols. We propose several results to safely compose security protocols (see Section IV). In particular, we consider parallel composition and the case of key-exchange protocols. Our results apply to confidentiality but also privacy-type properties (e.g. anonymity, unlinkability). All these results are actually derive from a generic composition result presented in Appendix C. This result allows one to map a trace of the composed protocol into a trace of a disjoint case (protocol where the sub-protocols do not share any data), and conversely. It can be seen as an extension of the result presented in [10] where only a mapping from the shared case to the disjoint case is provided (and not the converse). Moreover, we consider a richer process algebra than the one used in [10]. In particular, we are able to deal with protocols with else branches and to compose protocols that both rely on asymmetric primitives (i.e. asymmetric encryption and signature). In Section V, we illustrate the usefulness of our composition results on protocols from the $3 \mathrm{G}$ phone application.

\section{MODELS FOR SECURITY PROTOCOLS}

In this section, we introduce the calculus that is used for describing protocols. It is close to the applied pi calculus as defined in [17] even if we use a slightly different syntax and a non-compositional semantics. In particular, we consider an assignment operation to make explicit the data that are shared among different processes.

\section{A. Messages}

As usual in this kind of models, messages are modelled using an abstract term algebra. We assume an infinite set of names $\mathcal{N}$ of base type (which are used for representing keys, nonces, ...) and a set $\mathcal{C} h$ of names of channel type (which are used to name communication channels). We also consider a set of variables $\mathcal{X}$, and a signature $\Sigma$ consisting of a finite set of function symbols. We rely on a sort system for terms. The details of the sort system are unimportant, as long as the base type differs from the channel type. As in the applied pi calculus, we suppose that function symbols only operate on and return terms of base type.

Terms are defined as names, variables, and function symbols applied to other terms. Let $\mathrm{N} \subseteq \mathcal{N} \cup \mathcal{C} h$ and $\mathrm{X} \subseteq \mathcal{X}$, the set of terms built from $N$, and $X$ by applying function symbols in $\Sigma$ is denoted by $\mathcal{T}(\Sigma, N \cup \mathrm{X})$. Of course function symbol application must respect sorts and arities. We write $f v(u)$ (resp. $f n(u)$ ) for the set of variables (resp. names) occurring in a term $u$. A term is ground if it does not contain any variable.

To model algebraic properties of cryptographic primitives, we define an equational theory by a finite set $\mathrm{E}$ of equations $u=v$ with $u, v \in \mathcal{T}(\Sigma, \mathcal{X})$, i.e. $u, v$ do not contain names. We define ${ }_{E}$ to be the smallest equivalence relation on terms, that contains $E$ and that is closed under application of function symbols and substitutions of terms for variables.

Example 1: Consider the following signature $\Sigma_{\mathrm{DH}}$ :

$$
\{\text { aenc, adec, pk, g, f }\} \text {. }
$$

The function symbols adec, aenc of arity 2 represent asymmetric decryption and encryption. We denote by $\mathrm{pk}(s k)$ the public key associated to the private key $s k$. We consider two additional function symbols $f$ of arity 2 , and $g$ of arity 1 that will be used to model the Diffie-Hellman primitives. The equational theory $\mathrm{E}_{\mathrm{DH}}$ is defined relying on two equations:

$$
\operatorname{adec}(\operatorname{aenc}(x, \operatorname{pk}(y)), y)=x \text { and } \mathrm{f}(\mathrm{g}(x), y)=\mathrm{f}(\mathrm{g}(y), x) \text {. }
$$

Let $u_{1}=\operatorname{aenc}\left(\mathrm{f}\left(\mathrm{g}\left(r_{A}\right), r_{B}\right), \operatorname{pk}\left(s k_{B}\right)\right)$. We have that

$$
\operatorname{adec}\left(u_{1}, s k_{B}\right)={ }_{\mathrm{E}_{\mathrm{DH}}} \mathrm{f}\left(\mathrm{g}\left(r_{A}\right), r_{B}\right)={ }_{\mathrm{E}_{\mathrm{DH}}} \mathrm{f}\left(\mathrm{g}\left(r_{B}\right), r_{A}\right) .
$$

\section{B. Processes}

As in the applied pi calculus, we consider plain processes as well as extended processes in order to represent processes that have already evolved by e.g. disclosing some terms to the environment. Plain processes are defined by the following grammar:

$$
\begin{aligned}
& P, Q:=0 \quad \text { null } \\
& P \mid Q \quad \text { parallel } \\
& \text { new n.P restriction } \\
& ! P \quad \text { replication } \\
& {[x:=v] . P \quad \text { assignment }} \\
& \text { if } \varphi \text { then } P \text { else } Q \text { conditional } \\
& \text { in }(c, x) . P \quad \text { input } \\
& \text { out }(c, v) \cdot Q \quad \text { output }
\end{aligned}
$$

where $c$ is a name of channel type, $\varphi$ is a conjunction of tests of the form $u_{1}=u_{2}$ where $u_{1}, u_{2}$ are terms of base type, $x$ is a variable of base type, $v$ is a term of base type, and $n$ is a name of any type. Note that the terms that occur in $\varphi$ and $v$ may contain variables. The grammar is quite similar to the one of the applied pi calculus. We consider in addition an assignment operation that instantiates $x$ with a term $v$. We consider private channels but we do not allow channel passing.

Names and variables have scopes, which are delimited by restrictions, inputs, and assignment operations. We write $f v(P), b v(P), f n(P)$ and $b n(P)$ for the sets of free and bound variables, and free and bound names of a plain process $P$. 


$$
\begin{array}{rlrrr}
\left(\mathcal{E} ;\left\{\text { if } \varphi \text { then } Q_{1} \text { else } Q_{2}\right\} \uplus \mathcal{P} ; \Phi ; \sigma\right) & \stackrel{\tau}{\rightarrow} & \left(\mathcal{E} ; Q_{1} \uplus \mathcal{P} ; \Phi ; \sigma\right) & \text { if } u \sigma=_{\mathrm{E}} v \sigma \text { for each } u=v \in \varphi & \text { (THEN) } \\
\left(\mathcal{E} ;\left\{\text { if } \varphi \text { then } Q_{1} \text { else } Q_{2}\right\} \uplus \mathcal{P} ; \Phi ; \sigma\right) & \stackrel{\tau}{\rightarrow} & \left(\mathcal{E} ; Q_{2} \uplus \mathcal{P} ; \Phi ; \sigma\right) & \text { if } u \sigma \neq \mathrm{E} v \sigma \text { for some } u=v \in \varphi & \text { (ELSE) } \\
\left(\mathcal{E} ;\left\{\text { out }(c, u) . Q_{1} ; \text { in }(c, x) \cdot Q_{2}\right\} \uplus \mathcal{P} ; \Phi ; \sigma\right) & \stackrel{\tau}{\rightarrow} & \left(\mathcal{E} ; Q_{1} \uplus Q_{2} \uplus \mathcal{P} ; \Phi ; \sigma \cup\{x \mapsto u \sigma\}\right) & \text { (COMM) } \\
(\mathcal{E} ;\{[x:=v] . Q\} \uplus \mathcal{P} ; \Phi ; \sigma) & \stackrel{\tau}{\rightarrow} & (\mathcal{E} ; Q \uplus \mathcal{P} ; \Phi ; \sigma \cup\{x \mapsto v \sigma\}) & \text { (ASSGN) } \\
(\mathcal{E} ;\{\text { in }(c, z) . Q\} \uplus \mathcal{P} ; \Phi ; \sigma) & \stackrel{\text { in }(c, M)}{\longrightarrow} & (\mathcal{E} ; Q \uplus \mathcal{P} ; \Phi ; \sigma \cup\{z \mapsto u\}) & \text { if } c \notin \mathcal{E}, M \Phi=u, f v(M) \subseteq \operatorname{dom}(\Phi) \text { and } f n(M) \cap \mathcal{E}=\emptyset \\
& & \text { (IN) } \\
(\mathcal{E} ;\{\text { out }(c, u) . Q\} \uplus \mathcal{P} ; \Phi ; \sigma) & \stackrel{\nu w_{n} \text { out }\left(c, w_{n}\right)}{\longrightarrow} & \left(\mathcal{E} ; Q \uplus \mathcal{P} ; \Phi \cup\left\{w_{n} \triangleright u \sigma\right\} ; \sigma\right) & \text { (OUT-T) }
\end{array}
$$

if $c \notin \mathcal{E}, u$ is a term of base type, and $w_{n}$ is a variable such that $n=|\Phi|+1$

$$
\begin{array}{rll}
(\mathcal{E} ;\{\text { new } n . Q\} \uplus \mathcal{P} ; \Phi ; \sigma) & \stackrel{\tau}{\rightarrow} & (\mathcal{E} \cup\{n\} ; Q \uplus \mathcal{P} ; \Phi ; \sigma) \\
(\mathcal{E} ;\{! Q\} \uplus \mathcal{P} ; \Phi ; \sigma) & \stackrel{\tau}{\rightarrow} & (\mathcal{E} ;\{! Q ; Q \rho\} \uplus \mathcal{P} ; \Phi ; \sigma)
\end{array}
$$

where $\rho$ is used to rename variables in $b v(Q)$ (resp. names in $b n(Q)$ ) with fresh variables (resp. names)

$$
\left(\mathcal{E} ;\left\{P_{1} \mid P_{2}\right\} \uplus \mathcal{P} ; \Phi ; \sigma\right) \stackrel{\sim}{\rightarrow}\left(\mathcal{E} ;\left\{P_{1}, P_{2}\right\} \uplus \mathcal{P} ; \Phi ; \sigma\right)
$$

where $n$ is a name, $c$ is a name of channel type, $u, v$ are terms of base type, and $x, z$ are variables of base type.

Fig. 1. Semantics of extended processes

Example 2: Let $P_{\mathrm{DH}}=$ new $s k_{A}$.new $s k_{B} \cdot\left(P_{A} \mid P_{B}\right)$ a process that models the Diffie-Hellman key exchange protocol where processes $P_{A}$ and $P_{B}$ are as follows:

- $P_{A} \stackrel{\text { def }}{=}$ new $r_{A}$.out $\left(c, \operatorname{aenc}\left(\mathrm{g}\left(r_{A}\right), \operatorname{pk}\left(s k_{B}\right)\right)\right)$.in $\left(c, y_{A}\right)$. $\left[x_{A}:=\mathrm{f}\left(\operatorname{adec}\left(y_{A}, s k_{A}\right), r_{A}\right)\right] .0$

- $P_{B} \stackrel{\text { def }}{=}$ new $r_{B}$.out $\left(c, \operatorname{aenc}\left(\mathrm{g}\left(r_{B}\right), \operatorname{pk}\left(s k_{A}\right)\right)\right)$. in $\left(c, y_{B}\right)$. $\left[x_{B}:=\mathrm{f}\left(\operatorname{adec}\left(y_{B}, s k_{B}\right), r_{B}\right)\right] .0$.

The process $P_{A}$ generates a fresh random number $r_{A}$, sends the message aenc $\left(\mathrm{g}\left(r_{A}\right), \operatorname{pk}\left(s k_{B}\right)\right)$ on the public channel $c$, and waits for a message in order to compute his own view of the key that will be stored in $x_{A}$. The process $P_{B}$ does exactly the same. The computed value is stored in $x_{B}$.

Extended processes add a set of restricted names $\mathcal{E}$ (the names that are a priori unknown by the attacker), a sequence of messages $\Phi$ (corresponding to the messages that have been sent) and a substitution $\sigma$ which is used to store the messages that have been received as well as those that have been stored in assignment variables.

Definition 3: An extended process is $(\mathcal{E} ; \mathcal{P} ; \Phi ; \sigma)$ with:

- $\mathcal{E}$ a set of names that represents the names that are restricted in $\mathcal{P}, \Phi$ and $\sigma$

- $\mathcal{P}$ a multiset of plain processes with $f v(\mathcal{P}) \subseteq \operatorname{dom}(\sigma)$;

- $\Phi=\left\{w_{1} \triangleright u_{1}, \ldots, w_{n} \triangleright u_{n}\right\}$ where $u_{1}, \ldots, u_{n}$ are ground terms, and $w_{1}, \ldots, w_{n}$ are variables; and

- $\sigma=\left\{x_{1} \mapsto v_{1}, \ldots, x_{m} \mapsto v_{m}\right\}$ a substitution where $v_{1}, \ldots, v_{m}$ are ground terms, $x_{1}, \ldots, x_{m}$ are variables.

We write $b n(A)$ (resp. $f n(A)$ ) for the set of bound (resp. free) names of an extended process $A$. Given an extended process $A=(\mathcal{E} ; \mathcal{P} ; \Phi ; \sigma)$, we have that $b n(A)=b n(\mathcal{P}) \cup \mathcal{E}$ and $f n(A)=f n(\mathcal{P}, \Phi, \sigma) \backslash \mathcal{E}$. For sake of simplicity, we assume that extended processes are name and variable distinct: i.e. $b n(A) \cap f n(A)=b v(\mathcal{P}) \cap f v(\mathcal{P})=\emptyset$, any name and variable is at most bound once in $\mathcal{P}$, and $\mathcal{E} \cap b n(\mathcal{P})=\emptyset$.

For sake of clarity, we often omit brackets and the null process. For instance, we write $k_{1}$, and out $(c, u)$ instead of $\left\{k_{1}\right\}$ and $\{$ out $(c, u) .0\}$. When there is no "else", it means "else 0 ". Moreover, we often write $P$ instead of $(\emptyset ; P ; \emptyset ; \emptyset)$, or $(\mathcal{E} ; P ; \Phi)$ instead of $(\mathcal{E} ; P ; \Phi ; \emptyset)$.

The semantics is given by a set of labelled rules that allows one to reason about processes that interact with their environment (see Figure 1). This defines the relation $\stackrel{\ell}{\longrightarrow}$ where $\ell$ is either an input, an output, or a silent action $\tau$. The relation $\stackrel{\mathrm{tr}}{\longrightarrow}$ where $\mathrm{tr}$ denotes a sequence of labels is defined in the usual way whereas the relation $\stackrel{\operatorname{tr}^{\prime}}{\Longrightarrow}$ on processes is defined by: $A \stackrel{\mathrm{tr}^{\prime}}{\Longrightarrow} B$ if, and only if, there exists a sequence $\operatorname{tr}$ such that $A \stackrel{\text { tr }}{\longrightarrow} B$ and $\operatorname{tr}^{\prime}$ is obtained by erasing all occurrences of the silent action $\tau$ in tr.

Example 4: Let $\Phi_{\mathrm{DH}} \stackrel{\text { def }}{=}\left\{w_{1} \triangleright \mathrm{pk}\left(s k_{A}\right), w_{2} \triangleright \mathrm{pk}\left(s k_{B}\right)\right\}$, and $A_{\mathrm{DH}} \stackrel{\text { def }}{=}\left(\left\{s k_{A}, s k_{B}\right\} ; P_{A} \mid P_{B} ; \Phi_{\mathrm{DH}}\right)$ where $P_{A}$ and $P_{B}$ are as defined in Example 2. We have that:

$$
\begin{aligned}
& A_{\mathrm{DH}} \quad \stackrel{\tau}{\rightarrow} \stackrel{\tau}{\rightarrow} \stackrel{\tau}{\rightarrow} \\
& \stackrel{\nu w_{3} \text {.out }\left(c, w_{3}\right)}{\longrightarrow} \stackrel{\nu w_{4} \text {.out }\left(c, w_{4}\right)}{\longrightarrow}\left(\mathcal{E} ;\left\{Q_{A}, Q_{B}\right\} ; \Phi_{\mathrm{DH}} \uplus \Phi ; \emptyset\right) \\
& \stackrel{\text { in }\left(c, w_{3}\right)}{\longrightarrow} \stackrel{\text { in }\left(c, w_{4}\right)}{\longrightarrow} \stackrel{\tau}{\rightarrow} \stackrel{\tau}{\rightarrow}\left(\mathcal{E} ; 0 ; \Phi_{\mathrm{DH}} \uplus \Phi ; \sigma \cup \sigma^{\prime}\right) \stackrel{\text { def }}{=} A^{\prime}
\end{aligned}
$$

where:

- $\mathcal{E}=\left\{s k_{A}, s k_{B}, r_{A}, r_{B}\right\}$

- $\Phi=\left\{w_{3} \triangleright \operatorname{aenc}\left(\mathrm{g}\left(r_{A}\right), p k_{B}\right), w_{4} \triangleright \operatorname{aenc}\left(\mathrm{g}\left(r_{B}\right), p k_{A}\right)\right\}$;

- $Q_{A}=\operatorname{in}\left(c, y_{A}\right) \cdot\left[x_{A}:=\mathrm{f}\left(\operatorname{adec}\left(y_{A}, s k_{A}\right), r_{A}\right)\right] .0$;

- $Q_{B}=\operatorname{in}\left(c, y_{B}\right) \cdot\left[x_{B}:=\mathrm{f}\left(\operatorname{adec}\left(y_{B}, s k_{B}\right), r_{B}\right)\right] .0$;

- $\sigma=\left\{y_{A} \mapsto \operatorname{aenc}\left(\mathrm{g}\left(r_{B}\right), p k_{A}\right), y_{B} \mapsto \operatorname{aenc}\left(\mathrm{g}\left(r_{A}\right), p k_{B}\right)\right\}$;

- $\sigma^{\prime}=\mathrm{E}_{\mathrm{DH}}\left\{x_{A} \mapsto \mathrm{f}\left(\mathrm{g}\left(r_{B}\right), r_{A}\right), x_{B} \mapsto \mathrm{f}\left(\mathrm{g}\left(r_{A}\right), r_{B}\right)\right\}$.

The three first steps are performed using the rules NEW and PAR. Then, OUT-T, IN, and ASSGN are used in order to reach the extended process $A^{\prime}$. We use $p k_{A}$ (resp. $p k_{B}$ ) as a shortcut for $\mathrm{pk}\left(s k_{A}\right)$ (resp. $\mathrm{pk}\left(s k_{B}\right)$ ).

\section{Equivalence-based security properties}

We are particularly interested in privacy-type properties such as those studied in [18], [19], [20], and that can be 
expressed using a notion of equivalence. For instance, the notion of strong unlinkability as defined in [19] is satisfied if an observer cannot tell if the users can execute multiple times or at most once the protocol under study. This can be formalized using an equivalence between two situations: one where each user can execute the protocol multiple times, and one where each user can execute the protocol at most once.

We consider here the notion of trace equivalence. Intuitively, two protocols $P$ and $Q$ are in trace equivalence, denoted $P \approx Q$, if whatever the messages they received (built upon previously sent messages), the resulting sequences of messages are indistinguishable from the point of view of an outsider. Given an extended process $A$, we define its set of traces as follows:

$\operatorname{trace}(A)=\{(\operatorname{tr}$, new $\mathcal{E} . \Phi) \mid A \stackrel{\operatorname{tr}}{\Longrightarrow}(\mathcal{E} ; \mathcal{P} ; \Phi ; \sigma)$

for some process $(\mathcal{E} ; \mathcal{P} ; \Phi ; \sigma)\}$.

The sequence of messages $\Phi$ together with the set of restricted names $\mathcal{E}$ (those unknown to the attacker) is called a frame. We say that a term $u$ is deducible (modulo E) from a frame $\phi=$ new $\mathcal{E} . \Phi$, denoted new $\mathcal{E} . \Phi \vdash u$, when there exists a term $M$ (called a recipe) such that $f n(M) \cap \mathcal{E}=\emptyset$, $f v(M) \subseteq \operatorname{dom}(\Phi)$, and $M \Phi={ }_{\mathrm{E}} u$.

Two frames are indistinguishable when the attacker cannot detect the difference between the two situations they represent, that is, his ability to distinguish whether two recipes $M$ and $N$ produce the same term does not depend on the frame.

Definition 5: Two frames $\phi_{1}$ and $\phi_{2}$ with $\phi_{i}=$ new $\mathcal{E} . \Phi_{i}$ $(i \in\{1,2\})$ are statically equivalent, denoted by $\phi_{1} \sim \phi_{2}$, when $\operatorname{dom}\left(\Phi_{1}\right)=\operatorname{dom}\left(\Phi_{2}\right)$, and for all terms $M, N$ (called recipes) such that $f n(M, N) \cap \mathcal{E}=\emptyset$ and $f v(M, N) \subseteq$ $\operatorname{dom}\left(\Phi_{1}\right)$, we have that:

$$
M \Phi_{1}={ }_{\mathrm{E}} N \Phi_{1} \text {, if and only if, } M \Phi_{2}={ }_{\mathrm{E}} N \Phi_{2} .
$$

In the definition above, the names $\mathcal{E}$ are bound in $\phi_{i}$ and can be renamed. Moreover names that do not appear in $\Phi_{i}$ can be added or removed from $\mathcal{E}$. Assuming that two frames share the same set of restricted names is not a real limitation.

Example 6: Consider for instance the following frames:

$$
\begin{aligned}
& \Phi_{1}=\left\{w_{1} \triangleright \mathrm{g}\left(r_{A}\right), w_{2} \triangleright \mathrm{g}\left(r_{B}\right), w_{3} \triangleright \mathrm{f}\left(\mathrm{g}\left(r_{A}\right), r_{B}\right)\right\} \\
& \Phi_{2}=\left\{w_{1} \triangleright \mathrm{g}\left(r_{A}\right), w_{2} \triangleright \mathrm{g}\left(r_{B}\right), w_{3} \triangleright k\right\}
\end{aligned}
$$

Let $\mathcal{E}=\left\{r_{A}, r_{B}, k\right\}$. We have that new $\mathcal{E} . \Phi_{1} \sim$ new $\mathcal{E} . \Phi_{2}$ (considering the equational theory $\mathrm{E}_{\mathrm{DH}}$ ). This equivalence shows that the term $\mathrm{f}\left(\mathrm{g}\left(r_{A}\right), r_{B}\right)$ (the Diffie-Hellman key) is indistinguishable from a random key. This indistinguishability property holds even if the messages $\mathrm{g}\left(r_{A}\right)$ and $\mathrm{g}\left(r_{B}\right)$ that are exchanged by the two parties to build this key are known to the attacker.

Two processes are trace equivalent if, whatever the messages they sent and received, their frames are in static equivalence.

Definition 7: Let $A$ and $B$ be two extended processes, $A \sqsubseteq B$ if for every $(\operatorname{tr}, \phi) \in \operatorname{trace}(A)$, there exists $\left(\operatorname{tr}^{\prime}, \phi^{\prime}\right) \in$ $\operatorname{trace}(B)$ such that $\operatorname{tr}=\operatorname{tr}^{\prime}$ and $\phi \sim \phi^{\prime}$. We say that $A$ and $B$ are trace equivalent, denoted by $A \approx B$, if $A \sqsubseteq B$ and $B \sqsubseteq A$.
This notion of equivalence allows us to express many interesting privacy-type properties such as vote-privacy, strong versions of anonymity and/or unlinkability, etc.

\section{THE CONDITIONS TO SAFELY REASON IN A MODULAR WAY}

It is well-known that even if two protocols are secure in isolation, it is not possible to compose them in arbitrary ways still preserving their security. This has already been observed for different kinds of compositions (e.g. parallel [8], sequential [10]) and when studying standard security properties [9] (e.g. secrecy, authentication) and privacy-type properties [16].

\section{A. Some well-known difficulties}

Sharing primitives. The interactions between protocols arise from the fact that a protocol may reveal a shared key while the security of the other protocol relies on the secrecy of this key. Even without revealing shared keys, a protocol can be used as an oracle by another protocol to decrypt a message, and then compromise the security of the whole application. To avoid this kind of interactions, most of the composition results assume that protocols do not share any primitive or allow a list of standard primitives (e.g. signature, encryption) to be shared as long as they are tagged in different ways. In this paper, we consider the fixed common signature $\Sigma_{0}=$ $\left\{\right.$ sdec, senc, adec, aenc, pk, \langle\rangle$, \operatorname{proj}_{1}, \operatorname{proj}_{2}$, sign, check, vk, h\} equipped with the equational theory $E_{0}$, defined by the following equations:

$$
\begin{gathered}
\operatorname{sdec}(\operatorname{senc}(x, y), y)=x, \\
\operatorname{adec}(\operatorname{aenc}(x, \operatorname{pk}(y)), y)=x, \\
\operatorname{check}(\operatorname{sign}(x, y), \operatorname{vk}(y))=x \\
\left.\operatorname{proj}_{i}\left(\left\langle x_{1}, x_{2}\right\rangle\right)=x_{i} \text { with } i \in\{1,2\}\right)
\end{gathered}
$$

This allows us to model symmetric/asymmetric encryption, concatenation, signatures, and hash functions. We denote by $\mathrm{pk}(s k)$ (resp. vk $(s k)$ ) the public key (resp. verification key) associated to the private key $s k$. We allow protocols to both rely on $\Sigma_{0}$ provided that each application of aenc, senc, sign, and $h$ is tagged (using disjoint sets of tags for the two protocols), and adequate tests are performed when receiving a message to ensure that the tags are correct. Actually, we consider the same tagging mechanism as the one introduced in [16] (see Appendix A for more details). Here, we simply illustrate this tagging mechanism on our running example.

Example 8: In order to compose the protocol $P_{\mathrm{DH}}=$ new $s k_{A}$.new $s k_{B} \cdot\left(P_{A} \mid P_{B}\right)$ introduced in Example 2 with another one that also relies on the primitive aenc, we may consider a tagged version of this protocol. For this, we introduce two function symbols tag ${ }_{1}$ and untag $_{1}$, and the equation $\operatorname{untag}_{1}\left(\operatorname{tag}_{1}(x)\right)=x$ to model the interaction between these two symbols. The tagged version (using tag ${ }_{1}$ ) of $P_{A}$ is given below (with $u=\operatorname{adec}\left(y_{A}, s k_{A}\right)$ ):

$$
\left\{\begin{aligned}
\operatorname{new} r_{A} \cdot & \text { out }\left(c, \operatorname{aenc}\left(\operatorname{tag}_{1}\left(\mathrm{~g}\left(r_{A}\right)\right), \operatorname{pk}\left(s k_{B}\right)\right)\right) \\
& \text { in }\left(c, y_{A}\right) . \\
& \text { if } \operatorname{tag}\left(\operatorname{untag}_{1}(u)\right)=u \\
& \operatorname{then}\left[x_{A}:=\mathrm{f}\left(\operatorname{untag}_{1}(u), r_{A}\right)\right] .0
\end{aligned}\right.
$$


The tagged version (using $\operatorname{tag}_{1}$ ) of $P_{B}$ can be obtained in a similar way, and thus we easily derive a tagged version of $P_{\mathrm{DH}}$ (by putting the two processes in parallel).

Revealing shared key. Consider two protocols, one whose security relies on the secrecy of a shared key whereas the other protocol decides to reveal it. Such a situation will compromise the security of the whole application. It is therefore important to ensure that shared keys are not revealed. To formalise this hypothesis, and to express the sharing of long-term keys, we introduce the notion of composition context. A composition context $C$ is defined by the grammar:

$$
C:=\_\mid \text {new } n . C \mid ! C
$$

where $n$ is a name of base type.

Definition 9: Let $C$ be a composition context, $A$ be an extended process of the form $(\mathcal{E} ; C[P] ; \Phi)$, key $\in$ $\{n, \operatorname{pk}(n), \operatorname{vk}(n) \mid n$ occurs in $C\}$, and $c, s$ two fresh names. We say that $A$ reveals key when $(\mathcal{E} \cup\{s\} ; C[P \mid$ in $(c, x)$. if $x=k e y$ then out $(c, s)] ; \Phi) \stackrel{\operatorname{tr}}{\Longrightarrow}\left(\mathcal{E}^{\prime} ; \mathcal{P}^{\prime} ; \Phi^{\prime}\right)$ for some $\mathcal{E}^{\prime}, \mathcal{P}^{\prime}$, and $\Phi^{\prime}$ such that new $\mathcal{E}^{\prime} . \Phi^{\prime} \vdash s$.

We can now formalize the requirements discussed so far and needed to compose protocols.

Definition 10: Let $C$ be a composition context and $\mathcal{E}_{0}$ be a finite set of names of base type. Let $P$ and $Q$ be two plain processes together with their frames $\Phi$ and $\Psi$. We say that $P / \Phi$ and $Q / \Psi$ are composable under $\mathcal{E}_{0}$ and $C$ when $f v(P)=$ $f v(Q)=\emptyset, \operatorname{dom}(\Phi) \cap \operatorname{dom}(\Psi)=\emptyset$, and

1) $P$ (resp. $Q$ ) is built over $\Sigma_{\alpha} \cup \Sigma_{0}$ (resp. $\Sigma_{\beta} \cup \Sigma_{0}$ ), whereas $\Phi$ (resp. $\Psi$ ) is built over $\Sigma_{\alpha} \cup\{\mathrm{pk}, \mathrm{vk}\}$ (resp. $\left.\Sigma_{\beta} \cup\{\mathrm{pk}, \mathrm{vk}\}\right), \Sigma_{\alpha} \cap \Sigma_{\beta}=\emptyset$, and $P$ (resp. $Q$ ) is tagged;

2) $\mathcal{E}_{0} \cap(f n(C[P]) \cup f n(\Phi)) \cap(f n(C[Q]) \cup f n(\Psi))=\emptyset$; and

3) $\left(\mathcal{E}_{0} ; C[P] ; \Phi\right)$ (resp. $\left.\left(\mathcal{E}_{0} ; C[Q] ; \Psi\right)\right)$ does not reveal any key in

$\{n, \operatorname{pk}(n), \operatorname{vk}(n) \mid n$ occurs in $f n(P) \cap f n(Q) \cap b n(C)\}$.

Condition 1 is about sharing primitives, whereas Conditions 2 and 3 ensure that keys are shared via the composition context $C$ only (not via $\mathcal{E}_{0}$ ), and are not revealed by each protocol individually.

\section{B. Controlling data shared via assignment}

Our goal is to go beyond parallel composition, and we want to consider the particular case of key-exchange protocols. Assume that $P=$ new $\tilde{n} \cdot\left(P_{1} \mid P_{2}\right)$ is a protocol that establishes a key between two participants. The goal of $P$ is to establish a fresh shared session key between $P_{1}$ and $P_{2}$. Assume that $P_{1}$ will store the key in the variable $x_{1}$, while $P_{2}$ will store it in the variable $x_{2}$, and then consider a protocol $Q$ that will use the values stored in $x_{1} / x_{2}$ as a fresh key to secure communications. In this setting, sharing between $P$ and $Q$ is achieved through the composition context and also through assignment variables $x_{1}$ and $x_{2}$. The idea is to abstract these values with fresh names when we analyse $Q$ in isolation. However, in order to abstract them in the right way, we need to know their values (or at least whether they are equal or not). This is the purpose of the property stated below.
Definition 11: Let $C$ be a composition context and $\mathcal{E}_{0}$ be a finite set of names. Let $P_{1}\left[\_\right]$(resp. $P_{2}\left[\_\right.$) be a plain process with an hole in the scope of an assignment of the form $\left[x_{1}:=t_{1}\right]$ (resp. $\left[x_{2}:=t_{2}\right]$ ), and $\Phi$ be a frame. We say that $P_{1} / P_{2} / \Phi$ is a good key-exchange protocol under $\mathcal{E}_{0}$ and $C$ when $\left(\mathcal{E}_{0} ; P_{\text {good }} ; \Phi\right)$ does not reveal bad where $P_{\text {good }}$ is defined as follows:

$$
\begin{aligned}
& P_{\text {good }}=\text { new bad, } d .( \\
& C\left[\text { new } i d .\left(P_{1}\left[\operatorname{out}\left(d,\left\langle x_{1}, i d\right\rangle\right)\right] \mid P_{2}\left[\operatorname{out}\left(d,\left\langle x_{2}, i d\right\rangle\right)\right]\right)\right] \\
& \mid \text { in }(d, x) . \operatorname{in}(d, y) . \\
& \quad \text { if } \operatorname{proj}_{1}(x)=\operatorname{proj}_{1}(y) \wedge \operatorname{proj}_{2}(x) \neq \operatorname{proj}_{2}(y) \\
& \quad \text { then out }(c, b a d) \\
& \mid \text { in }(d, x) . \text { in }(d, y) . \\
& \quad \text { if } \operatorname{proj}{ }_{1}(x) \neq \operatorname{proj}_{1}(y) \wedge \operatorname{proj}_{2}(x)=\operatorname{proj}_{2}(y) \\
& \quad \text { then out }(c, b a d) \\
& \mid \text { in }(d, x) . \text { in }(c, z) . \\
& \quad \text { if } z \in\left\{\operatorname{proj}_{1}(x), \operatorname{pk}\left(\operatorname{proj}_{1}(x)\right), \operatorname{vk}\left(\operatorname{proj}_{1}(x)\right)\right\} \\
& \quad \text { then out }(c, b a d))
\end{aligned}
$$

where bad is a fresh name of base type, and $c, d$ are fresh names of channel type.

The expressions $u \neq v$ and $u \in\left\{v_{1}, \ldots, v_{n}\right\}$ used above are convenient notations that can be rigorously expressed using nested conditionals as expected. Roughly, the property expresses that two assignment variables are assigned to the same value if, and only if, they are the assignment variables of two associated instances of $P_{1}$ and $P_{2}$ (i.e. that share the same $i d$ ). In particular, two instances of the role $P_{1}$ (resp. $P_{2}$ ) cannot assign their variable with the same value (i.e. a fresh key is established at each session). We also need to ensure that the data shared through $x_{1} / x_{2}$ are not revealed. This is the purpose of the last line of process $P_{\text {good }}$.

When analysing $Q$ in isolation, the values stored in the assignment variables $x_{1} / x_{2}$ are abstracted by fresh names. Since $P$ and $Q$ share the common signature $\Sigma_{0}$, we need an additional hypothesis to ensure that in any execution, the values assigned to the different $x_{1} / x_{2}$ are not of the form $\left\langle u_{1}, u_{2}\right\rangle, \operatorname{pk}(u)$, or $\operatorname{vk}(u)$. These symbols are those of the common signature that are not tagged, thus abstracting them by a fresh name in $Q$ would not be safe (e.g. $Q$ can easily check whether a term is a pair or not).

Definition 12: An extended process $A$ satisfies the $a b$ stractability property if for any $(\mathcal{E} ; \mathcal{P} ; \Phi ; \sigma)$ such that $A \stackrel{\text { tr }}{\Longrightarrow}(\mathcal{E} ; \mathcal{P} ; \Phi ; \sigma)$, for any $x \in \operatorname{dom}(\sigma)$ which corresponds to an assignment variable, for any $u_{1}, u_{2}$, we have that $x \sigma \neq \mathrm{E}\left\langle u_{1}, u_{2}\right\rangle, x \sigma \neq_{\mathrm{E}} \mathrm{pk}\left(u_{1}\right), x \sigma \neq_{\mathrm{E}} \mathrm{vk}\left(u_{1}\right)$.

\section{Some extra issues with trace equivalence}

In this work, we would like to consider sequential composition w.r.t. privacy-type properties. In the particular case where $f v(P[0])=f v(Q)=f v\left(P^{\prime}[0]\right)=f v\left(Q^{\prime}\right)=\emptyset$, we would like to state a result like:

$$
\begin{gathered}
C[P[0]] \approx C^{\prime}\left[P^{\prime}[0]\right] \wedge C[Q] \approx C^{\prime}\left[Q^{\prime}\right] \\
\Longrightarrow \\
C[P[Q]] \approx C^{\prime}\left[P^{\prime}\left[Q^{\prime}\right]\right]
\end{gathered}
$$


where $C, C^{\prime}$ are two composition contexts. This roughly means that assuming that the equivalence property holds for the protocol $P$ and $Q$ individually, then we can consider the situation where $Q$ is executed "after" $P$ to be safe. However, even in a quite simple setting (the shared keys are not revealed, protocols do not share any primitives), such a sequential composition result does not hold.

Example 13: Let $C=C^{\prime}=$ new $k$ ! new $k_{1}$. ! new $k_{2}$. be a composition context, $Q=Q_{0}$ (yes, no), and $Q^{\prime}=$ $Q_{0}$ (no, yes) where:

$$
\begin{aligned}
& Q_{0}\left(z_{1}, z_{2}\right)=\operatorname{out}\left(c_{Q}, u\right) \text {.in }(x) \text {.if } x=u \text { then } 0 \\
& \quad \text { else if } \operatorname{proj}_{1}(\operatorname{sdec}(x, k))=k_{1} \text { then out }\left(c_{Q}, z_{1}\right) \\
& \quad \text { else out }\left(c_{Q}, z_{2}\right)
\end{aligned}
$$

and

$$
\begin{aligned}
& P\left[\_\right]=P^{\prime}\left[\_\right]=\operatorname{out}\left(c_{P}, u\right) \cdot\left(\_\mid \text {in }\left(c_{P}, x\right) \text {.if } x=u \text { then } 0\right. \\
& \text { else if } \operatorname{proj}(\operatorname{sdec}(x, k))=k_{1} \operatorname{then} \operatorname{out}\left(c_{P}, \text { ok }\right) \\
& \text { else out } \left.\left(c_{P}, \mathrm{ko}\right)\right)
\end{aligned}
$$

where $u=\operatorname{senc}\left(\left\langle k_{1}, k_{2}\right\rangle, k\right)$, and yes/no, ok/ko are public constants. We have $C[P[0]] \approx C^{\prime}\left[P^{\prime}[0]\right]$ and also that $C[Q] \approx C\left[Q^{\prime}\right]$. This latter equivalence is a non-trivial one. Intuitively, when $C[Q]$ will unfold its outermost !, then the process $C^{\prime}\left[Q^{\prime}\right]$ will mimic this step by unfolding its innermost !. This will allow it to react in the same way as $C[Q]$ in case encrypted messages are used to fill some input actions. However, we have that $C[P[Q]] \not \approx C^{\prime}\left[P^{\prime}\left[Q^{\prime}\right]\right]$. Even, if $P=P^{\prime}$, the presence of these processes give to the attacker some additional distinguishing power. In particular, through the outputs ok/ko outputted by $P / P^{\prime}$, the attacker will learn which ! has been unfolded. This result holds even if we rename function symbols so that protocols $P$ and $Q$ will not share any primitives.

In the example above, the problem is that the two equivalences we want to compose hold for different reasons, i.e. by unfolding the replications in a different and incompatible way. Thus, when the composed process $C[P[Q]]$ reached a point where $Q$ can be executed, on the other side, the process $Q^{\prime}$ is ready to be executed but the instance that is available is not the one that was used when establishing the equivalence $C[Q] \approx C^{\prime}\left[Q^{\prime}\right]$. Therefore, in order to establish equivalencebased properties in a modular way, we rely on a stronger notion of equivalence, namely diff-equivalence, that will ensure that the two "small" equivalences will be satisfied in a compatible way. Note that this problem does not arise when considering reachability properties and/or parallel composition.

Biprocesses and diff-equivalence. We consider pairs of processes, called biprocesses, that have the same structure and differ only in the terms and tests that they contain. Following the approach of [4], we introduce a special symbol diff of arity 2 in our signature. The idea will be to use this diff operator to indicate when the terms manipulated by the processes are different. Given a biprocess $B$, we define two processes $\mathrm{fst}(B)$ and $\operatorname{snd}(B)$ as follows: $\mathrm{fst}(B)$ is obtained by replacing each occurrence of $\operatorname{diff}\left(M, M^{\prime}\right)\left(\operatorname{resp} . \operatorname{diff}\left(\varphi, \varphi^{\prime}\right)\right)$ with $M$ (resp. $\varphi$ ), and similarly snd $(B)$ is obtained by replacing each occurrence of $\operatorname{diff}\left(M, M^{\prime}\right)$ (resp. $\operatorname{diff}\left(\varphi, \varphi^{\prime}\right)$ ) with $M^{\prime}$ (resp. $\left.\varphi^{\prime}\right)$.

The semantics of biprocesses is defined as expected via a relation $\stackrel{\ell}{\rightarrow}_{\text {bi }}$ that expresses when and how a biprocess may evolve. A biprocess reduces if, and only if, both sides of the biprocess reduce in the same way: a communication succeeds on both sides, a conditional has to be evaluated in the same way in both sides too. When the two sides of the biprocess reduce in different ways, the biprocess blocks. For instance, the else rule is as follows:

$$
\begin{aligned}
&\left(\mathcal{E} ;\left\{\text { if } \operatorname{diff}\left(\varphi_{L}, \varphi_{R}\right) \text { then } Q_{1} \text { else } Q_{2}\right\} \uplus \mathcal{P} ; \Phi ; \sigma\right) \\
& \stackrel{\tau}{\text { bi }}\left(\mathcal{E} ; Q_{2} \uplus \mathcal{P} ; \Phi ; \sigma\right)
\end{aligned}
$$

if $u_{L} \sigma \neq_{\mathrm{E}} v_{L} \sigma$ for some $u_{L}=v_{L} \in \varphi_{L}$, and $u_{R} \sigma \neq_{\mathrm{E}} v_{R} \sigma$ for some $u_{R}=v_{R} \in \varphi_{R}$.

The relation $\stackrel{\text { tr }}{\Longrightarrow}_{\text {bi }}$ on biprocesses is defined as for processes. This leads us to the notion of diff-equivalence which is stronger than the usual notion of trace equivalence.

Definition 14: An extended biprocess $B_{0}$ satisfies diffequivalence if for every $B=(\mathcal{E} ; \mathcal{P} ; \Phi ; \sigma)$ such that $B_{0} \stackrel{\text { tr }}{\Longrightarrow}$ bi $B$ for some trace $\operatorname{tr}$, we have that (i) new $\mathcal{E}$.fst $(\Phi) \sim$ new $\mathcal{E}$.snd $(\Phi)$ (ii) if fst $(B) \stackrel{\ell}{\rightarrow} A_{L}$ then there exists $B^{\prime}$ such that $B \stackrel{\ell}{\rightarrow}_{\mathrm{bi}} B^{\prime}$ and fst $\left(B^{\prime}\right)=A_{L}$ (and similarly for snd).

Note that, considering diff-equivalence instead of trace equivalence, Example 13 is not a counterexample anymore. Indeed, considering the biprocess $B=Q_{0}(\operatorname{diff}($ yes, no) $) \operatorname{diff}($ no, yes $))$, we have that $B$ does not satisfy diff-equivalence. The notions introduced so far on processes are extended as expected on biprocesses: the property has to hold on both fst $(B)$ and $\operatorname{snd}(B)$. Sometimes, we also say that $B$ is in trace equivalence instead of writing $\operatorname{fst}(B) \approx \operatorname{snd}(B)$.

\section{COMPOSITION RESUlTS}

We now present several composition results. They are actually shown relying on a generic composition theorem that works on traces which is technical and only presented in Appendix. In particular, we consider the case of key-exchange protocols under arbitrary composition contexts. We obtain the first composition result that is able to deal with privacy-type properties for this kind of sequential composition. We are also able to retrieve and generalise several existing composition results that have been obtained in past few years for parallel composition and/or secrecy property (e.g. [16], [10]).

\section{A. The case of parallel composition}

We first consider the case of parallel composition in presence of equivalence-based properties. This result is in the spirit of the one established in [16]. However, in order to be able to combine this composition result with the one stated in Theorem 17, we also adapt it to diff-equivalence.

Theorem 15: Let $C$ be a composition context and $\mathcal{E}_{0}$ be a finite set of names of base type. Let $P$ and $Q$ be two plain 
biprocesses together with their frames $\Phi$ and $\Psi$, and assume that $P / \Phi$ and $Q / \Psi$ are composable under $\mathcal{E}_{0}$ and $C$.

If $\left(\mathcal{E}_{0} ; C[P] ; \Phi\right)$ and $\left(\mathcal{E}_{0} ; C[Q] ; \Psi\right)$ satisfy diff-equivalence (resp. trace equivalence) then the biprocess $\left(\mathcal{E}_{0} ; C[P \mid Q] ; \Phi \uplus\right.$ $\Psi)$ satisfies diff-equivalence (resp. trace equivalence).

From the proof of the previous result, we retrieve the following well-known composition result w.r.t. secrecy property. This result is actually a generalisation of the result proved in [10]. We allow more primitives, and a richer process algebra (e.g. else branches, private channels).

Corollary 16: Under the same hypotheses as Theorem 15 with processes instead of biprocesses, and considering a name $s$ that occurs in $C$. If $\left(\mathcal{E}_{0} ; C[P] ; \Phi\right)$ and $\left(\mathcal{E}_{0} ; C[Q] ; \Psi\right)$ do not reveal $s$ then $\left(\mathcal{E}_{0} ; C[P \mid Q] ; \Phi \cup \Psi\right)$ does not reveal $s$.

\section{B. The case of key-exchange protocols}

We now consider the particular case of key-exchange protocols with composition under arbitrary composition contexts.

Theorem 17: Let $C$ be a composition context and $\mathcal{E}_{0}$ be a finite set of names of base type. Let $P_{1}\left[\right.$ _] (resp. $P_{2}\left[\_\right]$) be a plain biprocess without replication and with an hole in the scope of an assignment of the form $\left[x_{1}:=t_{1}\right]$ (resp. $\left[x_{2}:=t_{2}\right]$ ). Let $Q_{1}$ (resp. $Q_{2}$ ) be a plain biprocess such that $f v\left(Q_{1}\right) \subseteq\left\{x_{1}\right\}$ (resp. $f v\left(Q_{2}\right) \subseteq\left\{x_{2}\right\}$ ), and $\Phi$ and $\Psi$ be two frames. Let $P=P_{1}[0] \mid P_{2}[0]$ and $Q=$ new $k .\left[x_{1}:=\right.$ $k] .\left[x_{2}:=k\right] .\left(Q_{1} \mid Q_{2}\right)$ for some fresh name $k$, and assume that:

1) $P / \Phi$ and $Q / \Psi$ are composable under $\mathcal{E}_{0}$ and $C$;

2) $\left(\mathcal{E}_{0} ; C[Q] ; \Psi\right)$ does not reveal $k, \operatorname{pk}(k), \operatorname{vk}(k)$;

3) $\left(\mathcal{E}_{0} ; C[P] ; \Phi\right)$ satisfies the abstractability property; and

4) $P_{1} / P_{2} / \Phi$ is a good key-exchange protocol under $\mathcal{E}_{0}$ and $C$.

If $\left(\mathcal{E}_{0} ; C[P] ; \Phi\right)$ and $\left(\mathcal{E}_{0} ; C[Q] ; \Psi\right)$ satisfy diff-equivalence, then $\left(\mathcal{E}_{0} ; C\left[P_{1}\left[Q_{1}\right] \mid P_{2}\left[Q_{2}\right]\right] ; \Phi \uplus \Psi\right)$ satisfies diff-equivalence.

We also retrieve the following result which is actually a generalization of two theorems established in [10]. We consider arbitrary composition contexts, more primitives, and a richer process algebra.

Corollary 18: Under the same hypotheses as Theorem 17 with processes instead of biprocesses, and considering a name $s$ that occurs in $C$. If $\left(\mathcal{E}_{0} ; C[P] ; \Phi\right)$ and $\left(\mathcal{E}_{0} ; C[Q] ; \Psi\right)$ do not reveal $s$ then $\left(\mathcal{E}_{0} ; C\left[P_{1}\left[Q_{1}\right] \mid P_{2}\left[Q_{2}\right]\right] ; \Phi \uplus \Psi\right)$ does not reveal $s$.

\section{CAse Study: 3G MOBile Phones}

We look at confidentiality and privacy guarantees provided by the $A K A$ protocol and the Submit SMS procedure ( $S S M S$ ) when run in composition as specified by the $3 \mathrm{GPP}$ consortium in [2].

\section{A. Protocols description}

The $s S M S$ protocol allows a mobile station (MS) to send an SMS to another MS through a serving network (SN). The confidentiality of the sent SMS relies on a session key $C K$ established through the execution of the $A K A$ protocol between the MS and the $\mathrm{SN}$.

The $A K A$ protocol achieves mutual authentication between a MS and a SN, and allows them to establish a shared session key $C K$. The $A K A$ protocol consists in the exchange of two messages: the authentication request and the authentication response. The $A K A$ protocol as deployed in real $3 \mathrm{G}$ telecommunication systems presents a linkability attack [21], and thus we consider here its fixed version as described in [21]. At the end of a successful execution of this protocol, both parties should agree on a fresh ciphering key $C K$. This situation can be modelled in our calculus as follows:

new $s k_{S N}$. !new $I M S I$. new $k_{I M S I}$. !new sqn. new sms.

$$
\left(A K A^{S N}\left[s S M S^{S N}\right] \mid A K A^{M S}\left[s S M S^{M S}\right]\right)
$$

where $s k_{S N}$ represents the private key of the network; while $I M S I$ and $k_{I M S I}$ represent respectively the long-term identity and the symmetric key of the MS. The name sqn models the sequence number on which SN and MS are synchronised. The two subprocesses $A K A^{M S}$ and $s S M S^{M S}$ (resp. $A K A^{S N}$, and $s S M S^{S N}$ ) model one session of the MS's (resp. SN's) side of the $A K A$, and $s S M S$ protocols respectively. Each MS, identified by its identity $I M S I$ and its key $k_{I M S I}$, can run multiple times the $A K A$ protocol followed by the $s S M S$ protocol.

\section{B. Security Analysis}

We explain below how some confidentiality properties (weak and strong secrecy) and an unlinkability property of the $A K A$ protocol and the $S S M S$ procedure can be derived relying on Corollary 18 and Theorem 17 . We do not need to tag the protocols under study to perform our analysis since they do not share any primitive but the pairing operator. Note that the $A K A$ protocol can not be modelled in the calculus given in [10] due to the need of non-tivial else branches.

Strong unlinkability. This property requires that an observer does not see the difference between the two following scenarios: (i) a same mobile phone sends several SMSs; or (ii) multiple mobile phones send at most one SMS each. To model this requirement, we consider the composition context ${ }^{1}$ :

$$
\begin{aligned}
C_{U}\left[\_\right] \stackrel{\text { def }}{=} & \text { !new } I M S I_{1} \text {. new } k_{I M S I_{1}} . \\
& \text { !new } I M S I_{2} \text {. new } k_{I M S I_{2}} . \\
& \text { let } I M S I=\operatorname{diff}\left[I M S I_{1}, I M S I_{2}\right] \text { in } \\
& \text { let } k_{I M S I}=\operatorname{diff}\left[k_{I M S I 1}, k_{I M S I_{2}}\right] \text { in } \\
& \text { new } s q n . \text { new sms. }
\end{aligned}
$$

To check if the considered $3 \mathrm{G}$ protocols satisfy strong unlinkability, one needs to check if the following biprocess satisfies diff-equivalence:

$$
\left(s k_{S N} ; C_{U}\left[A K A^{S N}\left[s S M S^{S N}\right] \mid A K A^{M S}\left[s S M S^{M S}\right]\right] ; \Phi_{0}\right)
$$

where $\Phi_{0}=\left\{w_{1} \triangleright \operatorname{pk}\left(s k_{S N}\right)\right\}$.

Hypotheses (1-4) stated in Theorem 17 are satisfied, and thus this equivalence can be derived from the following two "smaller" diff-equivalences:

\footnotetext{
${ }^{1}$ We use let $x=M$ in $P$ to denote the process $P\{M / x\}$.
} 


$$
\begin{gathered}
\left(s k_{S N} ; C_{U}\left[A K A^{S N}[0] \mid A K A^{M S}[0]\right] ; \Phi_{0}\right), \text { and } \\
\left(s k_{S N} ; C_{U}^{\prime}\left[s S M S^{S N} \mid s S M S^{M S}\right] ; \emptyset\right) .
\end{gathered}
$$

where

$$
\begin{gathered}
C_{U}^{\prime}\left[\_\right] \stackrel{\text { def }}{=} C_{U}\left[\text { new } c k . \text { let } x c k_{S N}=c k\right. \text { in } \\
\text { let } \left.x c k_{M S}=c k \text { in } \_\right]
\end{gathered}
$$

Weak secrecy. This property requires that the sent/received SMS is not deducible by an outsider, and can be modelled using the context

$$
C_{W S}\left[{ }_{-}\right] \stackrel{\text { def }}{=} \text { !new } I M S I \text {. new } k_{I M S I} \text {. !new sqn.new sms._ }
$$

Note that the composition context $C_{W S}$ is the same as fst $\left(C_{U}\right)$ (up to some renaming), thus Hypotheses (1-4) of Corollary 18 also hold and we derive the weak secrecy property by simply analysing this property on $A K A$ and $S S M S$ in isolation.

Strong secrecy. This property means that an outsider should not be able to distinguish the situation where $s m s_{1}$ is sen$\mathrm{t} /$ received, from the situation where $s m s_{2}$ is sent /received, although he might know the content of $s m s_{1}$ and $s m s_{2}$. This can be modelled using the following composition context:

$$
\begin{aligned}
C_{S S}\left[\_\right] \stackrel{\text { def }}{=} \text { !new } I M S I . \text { new } k_{I M S I} . \text { !new sqn. } \\
\text { let } s m s=\operatorname{diff}\left[s m s_{1}, s m s_{2}\right] \text { in } \_
\end{aligned}
$$

where $s m s_{1}$ and $s m s_{2}$ are two free names known to the attacker. Again, our Theorem 17 allows us to reason about this property in a modular way.

\section{CONCLUSION}

We investigate composition results for reachability properties as well as privacy-type properties expressed using a notion of equivalence. Relying on a generic composition result $^{2}$ that allows one to strongly relate any trace of the composed protocol to a trace of the so-called disjoint case, we derive parallel composition results, as well as a sequential composition results (the case of key-exchange protocols under various composition contexts).

All these results work in a quite general setting, e.g. processes may have non trivial else branches, we consider arbitrary primitives expressed using an equational theory, and processes may even share some standard primitives as long as they are tagged in different ways. We illustrate the usefulness of our results through the mobile phone application.

We think that our generic result could be used to derive some other composition results. We may want for instance to relax the notion of being a good protocol at the price of studying a less ideal scenario when analysing the protocol $Q$ in isolation. We may also want to consider situations where subprotocols sharing some data are arbitrarily interleaved. Moreover, even if we consider arbitrary primitives, sub-protocols can only share some standard primitives provided that they are tagged. It would be nice to relax these conditions. This would allow one to compose protocols (and not their tagged

\footnotetext{
${ }^{2}$ Due to lack of space, this generic composition result that is a bit technical is only stated in Appendix C.
}

versions) or to compose protocols that both rely on primitives for which no tagging scheme actually exists (e.g. exclusiveor).

Acknowledgement: The research leading to these results has received funding from the European Research Council under the European Union's Seventh Framework Programme (FP7/2007-2013) / ERC grant agreement $n^{\circ}$ 258865, project ProSecure, as well as the ANR project JCJC VIP $\mathrm{n}^{\circ} 11 \mathrm{JS} 02$ 00601.

\section{REFERENCES}

[1] B. X. Chen, "How to shield yourself from smartphone snoops," the New York Times, April 3, 2013.

[2] 3GPP, "Technical specification group services and system aspects; 3G security; security architecture (release 9)," 3rd Generation Partnership Project, Tech. Rep., 2010, 3GPP TS 33.102 V9.3.0

[3] A. Armando, R. Carbone, L. Compagna, J. Cuéllar, and M. L. Tobarra, "Formal analysis of SAML 2.0 web browser single sign-on: breaking the SAML-based single sign-on for google apps," in Proc. 6th ACM Workshop on Formal Methods in Security Engineering (FMSE 2008). ACM Press, 2008, pp. 1-10.

[4] B. Blanchet, M. Abadi, and C. Fournet, "Automated verification of selected equivalences for security protocols," Journal of Logic and Algebraic Programming, vol. 75, no. 1, pp. 3-51, 2008.

[5] A. Armando et al., "The AVANTSSAR Platform for the Automated Validation of Trust and Security of Service-Oriented Architectures," in Proc. 18th International Conference on Tools and Algorithms for the Construction and Analysis of Systems TACAS'12, ser. LNCS, vol. 7214. Springer, 2012.

[6] V. Cheval, H. Comon-Lundh, and S. Delaune, "Trace equivalence decision: Negative tests and non-determinism," in Proceedings of the 18th ACM Conference on Computer and Communications Security (CCS'11). ACM Press, 2011, pp. 321-330.

[7] A. Tiu and J. E. Dawson, "Automating open bisimulation checking for the spi calculus," in Proc. 23rd Computer Security Foundations Symposium (CSF'10). IEEE Comp. Soc. Press, 2010.

[8] J. D. Guttman and F. J. Thayer, "Protocol independence through disjoint encryption." in Proc. 13th Computer Security Foundations Workshop (CSFW'00). IEEE Comp. Soc. Press, 2000.

[9] V. Cortier and S. Delaune, "Safely composing security protocols," Formal Methods in System Design, vol. 34, no. 1, pp. 1-36, Feb. 2009.

[10] Ş. Ciobâcă and V. Cortier, "Protocol composition for arbitrary primitives," in Proc. of the 23rd IEEE Computer Security Foundations Symposium (CSF'10). IEEE Computer Society Press, 2010, pp. 322 336.

[11] B. Barak, R. Canetti, J. Nielsen, and R. Pass, "Universally composable protocols with relaxed set-up assumptions," in Proc. 45th Symposium on Foundations of Computer Science (FOCS'04). IEEE Computer Society Press, 2004, pp. 186-195.

[12] R. Küsters and M. Tuengerthal, "Composition Theorems Without PreEstablished Session Identifiers," in Proc. 18th Conference on Computer and Communications Security (CCS 2011). ACM Press, 2011, pp. 41-50.

[13] F. Böhl and D. Unruh, "Symbolic universal composability," in CSF 2013. IEEE, 2013, preprint on IACR ePrint 2013/062.

[14] T. Groß and S. Mödersheim, "Vertical protocol composition," in Proc. 24th IEEE Computer Security Foundations Symposium, (CSF'11). IEEE Computer Society, 2011, pp. 235-250.

[15] S. Mödersheim and L. Viganò, "Secure pseudonymous channels," in Proc. 14th European Symposium on Research in Computer Security (ESORICS'09), ser. LNCS, vol. 5789. Springer, 2009, pp. 337-354.

[16] M. Arapinis, V. Cheval, and S. Delaune, "Verifying privacy-type properties in a modular way," in Proc. 25th IEEE Computer Security Foundations Symposium (CSF'12). IEEE Computer Society Press, 2012, pp. 95-109.

[17] M. Abadi and C. Fournet, "Mobile values, new names, and secure communication," in Proc. 28th Symposium on Principles of Programming Languages (POPL'01). ACM Press, 2001.

[18] S. Delaune, S. Kremer, and M. D. Ryan, "Verifying privacy-type properties of electronic voting protocols," Journal of Computer Security, no. 4, pp. 435-487, Jul. 2008. 
[19] M. Arapinis, T. Chothia, E. Ritter, and M. Ryan, "Analysing unlinkability and anonymity using the applied pi calculus," in Proc. 23 rd Computer Security Foundations Symposium (CSF'10). IEEE Computer Society Press, 2010, pp. 107-121.

[20] M. Bruso, K. Chatzikokolakis, and J. den Hartog, "Formal verification of privacy for RFID systems," in Proc. 23rd Computer Security Foundations Symposium (CSF'10). IEEE Comp. Soc. Press, 2010.

[21] M. Arapinis, L. I. Mancini, E. Ritter, M. Ryan, N. Golde, K. Redon, and R. Borgaonkar, "New privacy issues in mobile telephony: fix and verification," in ACM Conference on Computer and Communications Security, 2012, pp. 205-216.

[22] Y. Chevalier and M. Rusinowitch, "Combining intruder theories," in Proc. 32nd International Colloquium on Automata, Languages and Programming (ICALP'05), ser. LNCS, vol. 3580. Springer, 2005, pp. 639-651.

[23] N. Dershowitz and J.-P. Jouannaud, "Rewrite systems," in Handbook of Theoretical Computer Science. Elsevier, 1990, vol. B, ch. 6.

[24] F. Baader and K. U. Schulz, "Unification in the union of disjoint equational theories: Combining decision procedures." Journal of Symbolic Computation, vol. 21, no. 2, pp. 211-243, 1996.

[25] Y. Chevalier and M. Rusinowitch, "Combining intruder theories," INRIA, Tech. Rep. 5495, 2005.

[26] V. Cortier and S. Delaune, "Decidability and combination results for two notions of knowledge in security protocols," Journal of Automated Reasoning, 2012, to appear.

[27] V. Cheval, "Automatic verification of cryptographic protocols: privacytype properties," Thèse de doctorat, Laboratoire Spécification et Vérification, ENS Cachan, France, Dec. 2012. [Online]. Available: http://www.lsv.ens-cachan.fr/Publis/PAPERS/PDF/cheval-these12.pdf

\section{APPENDIX A}

\section{SHARING PRIMITIVES VIA TAGGING}

We recall in this section the tagging scheme as presented in [16]. However, since we would like to be able to iterate our composition results (in order to compose e.g. three protocols), we consider a fixed set of colors (not only two), and we allow a process to be colored with many colors. Actually, a colored process is a process with a color assigned to each of its action. This gives us enough flexibility to allow different kinds of compositions, and to iterate our composition results.

We consider a family of signatures $\Sigma_{1}, \ldots, \Sigma_{p}$ disjoint from each other and disjoint from $\Sigma_{0}$. In order to tag a process, we introduce a new family of signatures $\Sigma_{1}^{\mathrm{tag}}, \ldots, \Sigma_{p}^{\mathrm{tag}}$. For each $i \in\{1, \ldots, p\}$, we have that $\Sigma_{i}^{\mathrm{tag}}=\left\{\operatorname{tag}_{i}\right.$, untag $\left._{i}\right\}$ where $\operatorname{tag}_{i}$

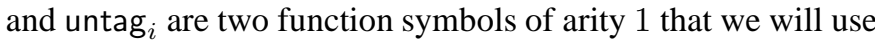
for tagging. The role of the $\operatorname{tag}_{i}$ function is to tag its argument

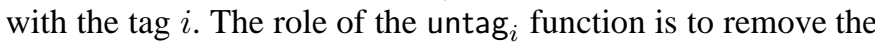
tag. To model this interaction between $\operatorname{tag}_{i}$ and untag $_{i}$, we consider the equational theory: $\mathrm{E}_{\operatorname{tag}_{i}}=\left\{\operatorname{untag}_{i}\left(\operatorname{tag}_{i}(x)\right)=\right.$ $x\}$.

For our composition result, we will assume that the two protocols we want to compose only share symbols in $\Sigma_{0}$. Thus, for this, we split the set $\{1, \ldots, p\}$ into two disjoint sets $\alpha$ and $\beta$. Given a subset $\gamma \subseteq\{1, \ldots, p\}$, we denote:

$$
\begin{gathered}
\Sigma_{\gamma} \stackrel{\text { def }}{=} \bigcup_{i \in \gamma} \Sigma_{i}, \quad \mathrm{E}_{\gamma} \stackrel{\text { def }}{=} \bigcup_{i \in \gamma} \mathrm{E}_{i}, \\
\Sigma_{\gamma}^{\text {tag }} \stackrel{\text { def }}{=} \bigcup_{i \in \gamma} \Sigma_{i}^{\text {tag }}, \quad \mathrm{E}_{\gamma}^{\text {tag }} \stackrel{\text { def }}{=} \bigcup_{i \in \gamma} \mathrm{E}_{i}^{\text {tag }}, \text { and } \\
\Sigma_{\gamma}^{+} \stackrel{\text { def }}{=} \Sigma_{\gamma} \cup \Sigma_{\gamma}^{\text {tag }}, \quad \mathrm{E}_{\gamma}^{+} \stackrel{\text { def }}{=} \mathrm{E}_{\gamma} \cup \mathrm{E}_{\gamma}^{\text {tag }} .
\end{gathered}
$$

Definition 19: Let $i \in\{1, \ldots, p\}$, and $u$ be a term built on $\Sigma_{i} \cup \Sigma_{0}$. The $i$-tagged version of $u$, denoted $[u]_{i}$ is defined as follows:

$$
\begin{aligned}
& {[\operatorname{senc}(u, v)]_{i} \stackrel{\text { def }}{=} \operatorname{senc}\left(\operatorname{tag}_{i}\left([u]_{i}\right),[v]_{i}\right)} \\
& {[\operatorname{aenc}(u, v)]_{i} \stackrel{\text { def }}{=} \operatorname{aenc}\left(\operatorname{tag}_{i}\left([u]_{i}\right),[v]_{i}\right)} \\
& {[\operatorname{sign}(u, v)]_{i} \stackrel{\text { def }}{=} \operatorname{sign}\left(\operatorname{tag}_{i}\left([u]_{i}\right),[v]_{i}\right)} \\
& {[\mathrm{h}(u)]_{i} \stackrel{\text { def }}{=} \mathrm{h}\left(\operatorname{tag}_{i}\left([u]_{i}\right)\right)} \\
& {[\operatorname{sdec}(u, v)]_{i} \stackrel{\text { def }}{=} \operatorname{untag}_{i}\left(\operatorname{sdec}\left([u]_{i},[v]_{i}\right)\right)} \\
& {[\operatorname{adec}(u, v)]_{i} \stackrel{\text { def }}{=} \operatorname{untag}_{i}\left(\operatorname{adec}\left([u]_{i},[v]_{i}\right)\right)} \\
& {[\operatorname{check}(u, v)]_{i} \stackrel{\text { def }}{=} \operatorname{untag}_{i}\left(\operatorname{check}\left([u]_{i},[v]_{i}\right)\right)} \\
& {\left[\mathrm{f}\left(u_{1}, \ldots, u_{n}\right)\right]_{i} \stackrel{\text { def }}{=} \mathrm{f}\left(\left[u_{1}\right]_{i}, \ldots,\left[u_{n}\right]_{i}\right) \text { otherwise. }}
\end{aligned}
$$

Note that we do not tag the pairing function symbol (this is actually useless), and we do not tag the pk and vk function symbols. Note that tagging pk and vk would lead us to consider an unrealistic modelling for asymmetric keys. This definition is extended as expected to formulas $\varphi$ (those involved in conditionals) by applying the transformation on each term that occurs in $\varphi$.

Example 20: Let $\Sigma_{1}=\{\mathrm{f}, \mathrm{g}\}$, and consider the terms $u=$ $\operatorname{senc}(\mathrm{g}(r), k)$ and $v=\mathrm{f}(\operatorname{sdec}(y, k), r)$ built on $\Sigma_{1} \cup \Sigma_{0}$. We have that:

- $[u]_{1}=\operatorname{senc}\left(\operatorname{tag}_{1}(\mathrm{~g}(r)), k\right)$, and

- $[v]_{1}=\mathrm{f}\left(\operatorname{untag}_{1}(\operatorname{sdec}(y, k)), r\right)$.

We also introduce the following notion that allows us to associate a color to a term that is not necessarily well-tagged.

Definition 21: Let $u$ be a term. We define tagroot $(u)$, namely the tag of the root of $u$ as follows:

- $\operatorname{tag} \operatorname{root}(u)=\perp$ when $u \in \mathcal{N} \cup \mathcal{X}$;

- tagroot $(u)=i$ if $u=\mathrm{f}\left(u_{1}, \ldots, u_{n}\right)$ and either $\mathrm{f} \in \Sigma_{i} \cup$ $\Sigma_{i}^{\text {tag }}$, or $\mathrm{f} \in\{$ senc, aenc, sign, $\mathrm{h}\}$ and $u_{1}=\operatorname{tag}_{i}\left(u_{1}^{\prime}\right)$ for some $u_{1}^{\prime}$.

- $\operatorname{tag} \operatorname{root}(u)=0$ otherwise.

Before extending the notion of tagging to processes, we have to express the tests that are performed by an agent when he receives a message that is supposed to be tagged. This is the purpose of test ${ }_{i}(u)$ that represents the tests which ensure that every projection and every untagging performed by an agent during the computation of $u$ is successful.

Definition 22: Let $i \in\{1, \ldots, p\}$, and $u$ be a term built on $\Sigma_{i}^{+} \cup \Sigma_{0}$. We define $\operatorname{test}_{i}(u)$ as follows:

$$
\begin{array}{r}
\operatorname{test}_{i}(u) \stackrel{\text { def }}{=} \operatorname{test}_{i}\left(u_{1}\right) \wedge \operatorname{test}_{i}\left(u_{2}\right) \wedge \operatorname{tag}_{i}\left(\operatorname{untag}_{i}(u)\right)=u \\
\text { when } u=\mathrm{g}\left(u_{1}, u_{2}\right) \text { with } \mathrm{g} \in\left\{\operatorname{sdec}, \operatorname{adec}_{\text {check }}\right\} \\
\operatorname{test}_{i}(u) \stackrel{\text { def }}{=} \operatorname{test}_{i}\left(u_{1}\right) \wedge u_{1}=\left\langle\operatorname{proj}_{1}\left(u_{1}\right), \operatorname{proj}_{2}\left(u_{1}\right)\right\rangle \\
\operatorname{test}_{i}(u) \stackrel{\text { def }}{=} \operatorname{true}_{\operatorname{dest}_{i}(u)} \stackrel{\text { when } u=\operatorname{proj}_{j}\left(u_{1}\right) \text { with } j \in\{1,2\}}{=} \operatorname{test}_{i}\left(u_{1}\right) \wedge \ldots \wedge \text { test }_{i}\left(u_{n}\right) \quad \text { when } u \text { is a name or a variable }
\end{array}
$$

This definition is extended as expected to formulas $\varphi$, i.e.

$$
\operatorname{test}_{i}(\varphi) \stackrel{\text { def }}{=} \bigwedge_{u=v \in \varphi} \operatorname{test}_{i}(u) \wedge \operatorname{test}_{i}(v) .
$$


Example 23: Again, consider $u=\operatorname{senc}(\mathrm{g}(r), k)$ and $v=$ $\mathrm{f}(\operatorname{sdec}(y, k), r)$. We have that:

$$
\begin{aligned}
& \operatorname{test}_{1}\left([u]_{1}\right)=\operatorname{true} \\
& \operatorname{test}_{1}\left([v]_{1}\right)=\operatorname{tag}_{1}\left(\operatorname{untag}_{1}(\operatorname{sdec}(y, k))\right)=\operatorname{sdec}(y, k)
\end{aligned}
$$

We consider colored plain processes meaning that initially the actions of a plain process will be annotated with a color, i.e. an integer in $\{1, \ldots, p\}$. The actions that need to be annotated are those that involve some composed terms, i.e. inputs, outputs, conditionals, and assignments. An action colored by $i \in\{1, \ldots, p\}$ can only contain function symbol from $\Sigma_{i}$. Given a set $\gamma \subseteq\{1, \ldots, p\}$, we say than an action is colored with $\gamma$ if this action is colored by $i \in\{1, \ldots, p\}$. For colored plain processes, the transformation $\llbracket P \rrbracket$ is defined as follows:

$$
\begin{aligned}
& \llbracket 0 \rrbracket \stackrel{\text { def }}{=} 0 \quad \llbracket ! P \rrbracket \stackrel{\text { def }}{=} ! \llbracket P \rrbracket \quad \text { new } k \cdot P \rrbracket \stackrel{\text { def }}{=} \text { new } k \cdot \llbracket P \rrbracket \\
& \llbracket P|Q \rrbracket \stackrel{\text { def }}{=} \llbracket P \rrbracket| \llbracket Q \rrbracket \quad \llbracket \text { in }(u, x)^{i} \cdot P \rrbracket \stackrel{\text { def }}{=} \text { in }(u, x)^{i} \cdot \llbracket P \rrbracket \\
& \llbracket[x:=v]^{i} \cdot P \rrbracket \stackrel{\text { def }}{=}\left(\text { if } \operatorname{test}_{i}\left([v]_{i}\right) \quad \text { then }\left[x:=[v]_{i}\right]^{i} \cdot \llbracket P \rrbracket\right)^{i} \\
& \llbracket \text { out }(u, v)^{i} \cdot Q \rrbracket \stackrel{\text { def }}{=}\left(\text { if test }{ }_{i}\left([v]_{i}\right) \text { then out }\left(u,[v]_{i}\right)^{i} \cdot \llbracket Q \rrbracket\right)^{i} \\
& \llbracket(\text { if } \varphi \text { then } P \text { else } Q)^{i} \rrbracket \stackrel{\text { def }}{=} \\
& \begin{array}{r}
\text { if } \left.\varphi_{\text {test }} \text { then }\left(\text { if }[\varphi]_{i} \text { then } \llbracket P \rrbracket \text { else } \llbracket Q \rrbracket\right)\right)^{i} \\
\text { else } 0)^{i}
\end{array}
\end{aligned}
$$

Roughly, instead of simply outputting a term $v$, a process will first performed some tests to check that the term is correctly tagged and he will output its $i$-tagged version $[v]_{i}$. For an assignment, we will also check that the term is correctly tagged. For a conditional, the process will first check that the terms involved in the test $\varphi$ are correctly tagged before checking that the test is satisfied. The annotations that occur on a plain process do not affect its semantics.

Definition 24: Consider a set $\gamma \subseteq\{1, \ldots, p\}$. Consider a plain process $P$ built over $\Sigma_{\gamma}^{+} \cup \Sigma_{0}$. We say that $P$ is tagged if there exists a colored plain process $Q$ built over $\Sigma_{\gamma}$ such that $P=\llbracket Q \rrbracket$.

\section{APPENDIX B \\ BIPROCESSES}

The semantics of biprocesses is defined via a relation $\stackrel{\ell}{\rightarrow}_{\text {bi }}$ that expresses when and how a biprocess may evolve. Intuitively, a biprocess reduces if and only if both sides of the biprocess reduce in the same way: a communication succeeds on both sides, a conditional has to be evaluated in the same way in both sides too. When the two sides of the biprocess reduce in different ways, the biprocess blocks. The semantics of biprocesses is formally described in Figure 2.

\section{APPENDIX C}

\section{THE DISJOINT CASE FOR A TRACE}

Composition usually works well in the so-called disjoint case, i.e. when the protocols under study do not share any secrets. The goal of this section is to show that we can map any trace corresponding to an execution of a protocol (with some sharing) to another trace which corresponds to an execution of a "disjoint case" (where protocols do not share any secrets) preserving static equivalence. We need a strong mapping to ensure that processes evolve simultaneously, and we rely for this on the notion of biprocesses.

We will see in this section that the composition of processes sharing some secrets (the so-called shared case) behaves as if they did not share any secret (the so-called disjoint case), provided that the shared secrets are never revealed and processes are tagged.

\section{A. Material for combination}

We denote by root(.) the function that associates to each term $M \in \mathcal{T}(\Sigma, \mathcal{N} \cup \mathcal{X})$ the function symbol at position $\epsilon$ (root position) in $M$. For $M \in \mathcal{N} \cup \mathcal{X}$, we $\operatorname{define} \operatorname{root}(M)=\perp$, where $\perp$ is a new symbol. The term $N$ is alien to $M$ if $\operatorname{root}(N) \in \Sigma_{i}, \operatorname{root}(M) \in \Sigma_{j}$ and $i \neq j$. We now introduce our notion of factors. A similar notion is also used in [22].

Definition 25 (factors): Let $M \in \mathcal{T}(\Sigma, \mathcal{N} \cup \mathcal{X})$. The factors of $M$, denoted $F c t(M)$, are the maximal syntactic subterms of $M$ that are alien to $M$

To handle the different signatures and equational theories, we consider the notion of ordered rewriting. defined in [23], which is a useful tool that has been used (e.g. [24]) for proving correctness of combination of unification algorithms. Most of the definitions and results in this subsection are borrowed from [25] and [26] since we use similar techniques. Let $\prec$ be a simplification ordering ${ }^{3}$ on ground terms assumed to be total and such that the minimum for $\prec$ is a name $n_{\text {min }}$ and the constants in $\Sigma$ are smaller that any ground term that is neither a constant nor a name. We define $\tilde{\Sigma}$ to be the set of the function symbols of $\Sigma_{1}, \ldots, \Sigma_{p}$ plus the name $n_{\text {min }}$, i.e. $\tilde{\Sigma}=\Sigma_{1} \cup \cdots \cup \Sigma_{p} \cup\left\{n_{\min }\right\}$. In what follows, we furthermore assume that $n_{\min }$ is never used under restriction in frames.

Given a possibly infinite set of equations $\mathcal{O}$ we define the ordered rewriting relation $\rightarrow_{\mathcal{O}}$ by $M \rightarrow_{\mathcal{O}} M^{\prime}$ if and only if there exists an equation $N_{1}=N_{2} \in \mathcal{O}$, a position $q$ in $M$ and a substitution $\tau$ such that:

$$
M=M\left[N_{1} \tau\right]_{q}, \quad M^{\prime}=M\left[N_{2} \tau\right]_{q} \text { and } N_{2} \tau \prec N_{1} \tau .
$$

It has been shown (see [23]) that by applying the unfailing completion procedure to a set of equations $\mathrm{E}$ we can derive a (possibly infinite) set of equations $\mathcal{O}$ such that on ground terms:

1) the relations $=_{\mathcal{O}}$ and $=_{\mathrm{E}}$ are equal,

2) the rewriting system $\rightarrow_{\mathcal{O}}$ is convergent.

It was showed ([24]) that applying unfailing completion to two disjoints sets of equations $E=E_{1} \cup E_{2}$, yields the set of generated equations $\mathcal{O}$ that is the disjoint union of the two systems $\mathcal{O}_{1}$ and $\mathcal{O}_{2}$ obtained by applying unfailing completion procedures to $E_{1}$ and to $E_{2}$ respectively. Thus, we can easily

\footnotetext{
${ }^{3}$ By definition $\prec$ satisfies that for all ground terms $M, N_{1}, N_{2}$, and for any position $q \neq \epsilon$ in $M$, we have $N_{1} \prec M\left[N_{1}\right]_{q}$ and $N_{1} \prec N_{2}$ implies $M\left[N_{1}\right]_{q} \prec M\left[N_{2}\right]_{q}$.
} 


$$
\begin{aligned}
& \left(\mathcal{E} ;\left\{\text { if } \operatorname{diff}\left(\varphi_{L}, \varphi_{R}\right) \text { then } Q_{1} \text { else } Q_{2}\right\} \uplus \mathcal{P} ; \Phi ; \sigma\right) \stackrel{\tau^{\prime}}{\text { bi }} \quad\left(\mathcal{E} ; Q_{1} \uplus \mathcal{P} ; \Phi ; \sigma\right) \quad \text { (THEN) } \\
& \text { if } u \sigma=\mathrm{E} v \sigma \text { for each } u=v \in \varphi_{L} \cup \varphi_{R} \\
& \left(\mathcal{E} ;\left\{\text { if } \operatorname{diff}\left(\varphi_{L}, \varphi_{R}\right) \text { then } Q_{1} \text { else } Q_{2}\right\} \uplus \mathcal{P} ; \Phi ; \sigma\right) \stackrel{\tau}{\rightarrow} \text { bi } \quad\left(\mathcal{E} ; Q_{2} \uplus \mathcal{P} ; \Phi ; \sigma\right) \quad \text { (ELSE) } \\
& \text { if } u_{L} \sigma \neq \mathrm{E} v_{L} \sigma \text { for some } u_{L}=v_{L} \in \varphi_{L} \text { and } u_{R} \sigma \neq_{\mathrm{E}} v_{R} \sigma \text { for some } u_{R}=v_{R} \in \varphi_{R} \\
& \left(\mathcal{E} ;\left\{\text { out }(c, u) . Q_{1} ; \operatorname{in}(c, x) \cdot Q_{2}\right\} \uplus \mathcal{P} ; \Phi ; \sigma\right) \quad \stackrel{\tau}{\rightarrow}_{\text {bi }} \quad\left(\mathcal{E} ; Q_{1} \uplus Q_{2} \uplus \mathcal{P} ; \Phi ; \sigma \cup\{x \mapsto u \sigma\}\right) \\
& (\mathcal{E} ;\{[x:=v] . Q\} \uplus \mathcal{P} ; \Phi ; \sigma) \quad \stackrel{\tau}{\rightarrow}_{\mathrm{bi}} \quad(\mathcal{E} ; Q \uplus \mathcal{P} ; \Phi ; \sigma \cup\{x \mapsto v \sigma\}) \\
& (\mathcal{E} ;\{\operatorname{in}(c, z) . Q\} \uplus \mathcal{P} ; \Phi ; \sigma) \quad \stackrel{\text { in }(c, M)}{\longrightarrow}_{\text {bi }} \quad(\mathcal{E} ; Q \uplus \mathcal{P} ; \Phi ; \sigma \cup\{z \mapsto u\}) \\
& (\mathcal{E} ;\{\text { out }(c, u) . Q\} \uplus \mathcal{P} ; \Phi ; \sigma) \quad \stackrel{\nu w_{n} \text {.out }\left(c, w_{n}\right)}{\longrightarrow} b_{\text {bi }} \quad\left(\mathcal{E} ; Q \uplus \mathcal{P} ; \Phi \cup\left\{w_{n} \triangleright u \sigma\right\} ; \sigma\right) \quad \text { (OUT-T) } \\
& \text { if } c \notin \mathcal{E}, u \text { is a term of base type, and } w_{n} \text { is a variable such that } n=|\Phi|+1 \\
& (\mathcal{E} ;\{\text { new } n . Q\} \uplus \mathcal{P} ; \Phi ; \sigma) \quad \stackrel{\tau}{\rightarrow}_{\text {bi }} \quad(\mathcal{E} \cup\{n\} ; Q \uplus \mathcal{P} ; \Phi ; \sigma) \\
& (\mathcal{E} ;\{! Q\} \uplus \mathcal{P} ; \Phi ; \sigma) \quad \stackrel{\tau}{\rightarrow}_{\mathrm{bi}} \quad(\mathcal{E} ;\{! Q ; Q \rho\} \uplus \mathcal{P} ; \Phi ; \sigma) \\
& \left(\mathcal{E} ;\left\{P_{1} \mid P_{2}\right\} \uplus \mathcal{P} ; \Phi ; \sigma\right) \quad \stackrel{\tau}{\rightarrow}_{\text {bi }} \quad\left(\mathcal{E} ;\left\{P_{1}, P_{2}\right\} \uplus \mathcal{P} ; \Phi ; \sigma\right)
\end{aligned}
$$

where $n$ is a name, $c$ is a name of channel type (here we can have $c=\operatorname{diff}\left(c_{1}, c_{2}\right)$ ), $u, v$ are terms that may contain the diff operator, and $x, z$ are variables. The term $M$ used in the IN rule is a term that does not contain any occurrence of the diff operator. The attacker has to do the same computation in both sides.

Fig. 2. Semantics for biprocesses

extend this result to $\mathrm{E}=\mathrm{E}_{1} \cup \ldots \cup \mathrm{E}_{p}$ since $\mathrm{E}_{1}, \ldots, \mathrm{E}_{p}$ are all disjoint two at a time.

Thus, applying unfailing completion to $\mathrm{E}=\mathrm{E}_{1} \cup \ldots \cup \mathrm{E}_{p}$ yields the set of generated equations $\mathcal{O}$ that is the disjoint union of $\mathcal{O}_{1}, \ldots, \mathcal{O}_{p}$ obtained by applying unfailing completion procedures respectively to $\mathrm{E}_{1}, \ldots, \mathrm{E}_{p}$.

Since the relation $\rightarrow_{\mathcal{O}}$ is convergent on ground terms, we define $M \downarrow_{E}$ (or briefly $M \downarrow$ ) as the unique normal form of the ground term $M$ for $\rightarrow_{\mathcal{O}}$. We denote by $M \downarrow_{\mathrm{E}_{i}}(i \in\{1, \ldots, p\})$ the unique normal form of the ground term $M$ for $\rightarrow \mathcal{O}_{i}$. These notations are extended as expected to sets of terms.

Lemma 26: Let $M$ be a ground term such that all its factors are in normal form and $\operatorname{root}(M) \in \Sigma_{i}$. Then

- either $M \downarrow \in F c t(M) \cup\left\{n_{\min }\right\}$,

- or $\operatorname{root}(M \downarrow) \in \Sigma_{i}$ and $F c t(M \downarrow) \subseteq F c t(M) \cup\left\{n_{m i n}\right\}$.

Lemma 27: Let $t$ be a ground term with $t=C_{1}\left[u_{1}, \ldots, u_{n}\right]$ where $C_{1}$ is a context built on $\Sigma_{i}, i \in\{1, \ldots, p\}$ and the terms $u_{1}, \ldots, u_{n}$ are the factors of $t$ in normal form. Let $C_{2}$ be a context built on $\Sigma_{i}$ (possibly a hole) such that $t \downarrow=$ $C_{2}\left[u_{j_{1}}, \ldots, u_{j_{k}}\right]$ with $j_{1}, \ldots, j_{k} \in\{0 \ldots n\}$ and $u_{0}=n_{\min }$ (the existence is given by Lemma 26). We have that for all ground terms $v_{1}, \ldots, v_{n}$ in normal form and alien to $t$, if

for every $q, q^{\prime} \in\{1 \ldots n\}$ we have $u_{q}=u_{q^{\prime}} \Leftrightarrow v_{q}=v_{q^{\prime}}$ then $C_{1}\left[v_{1}, \ldots, v_{n}\right] \downarrow=C_{2}\left[v_{j_{1}}, \ldots, v_{j_{k}}\right]$ with $v_{0}=n_{\text {min }}$.

A proof of these lemmas can be found in [26], [27].

\section{B. Generic composition result}

We consider two sets $\alpha, \beta$ such that $\alpha \cup \beta=\{1, \ldots, p\}$ and $\alpha \cap \beta=\emptyset$. We consider a plain colored process $P$ built on $\Sigma_{\alpha} \cup$
$\Sigma_{\beta} \cup \Sigma_{0}$ without replication and such that $b n(P)=f v(P)=\emptyset$. This means that $P$ is a process with no free variable, and we assume that it contains no name restrictions (i.e. no new instructions).

Example 28: We consider the process $P_{\mathrm{DH}}$ as given in Example 2 but we replace

- the 0 at the end of $P_{A}$ with $Q_{A}=$ new $s_{A}$. $\operatorname{out}\left(c, \operatorname{senc}_{D H}\left(s_{A}, x_{A}\right)\right)$, and

- the 0 at the end of $P_{B}$ with $Q_{B}=$ new $s_{B}$. $\operatorname{out}\left(c, \operatorname{senc}_{D H}\left(s_{B}, x_{B}\right)\right)$.

Intuitively, once the Diffie-Hellman key has been established and stored in $x_{A}$ (resp. $x_{B}$ ), each participant will use it to encrypt a fresh secret, namely $s_{A}$ or $s_{B}$, and then send it to the other participant.

To avoid confusion between the encryption schemes that processes can share, i.e. the function symbols in $\Sigma_{0}$, and the asymmetric encryption used in $P_{\mathrm{DH}}$ but not used in $Q_{A}$ and $Q_{B}$, we will rename them by aenc ${ }_{D H}$, $\operatorname{adec}_{D H}, \mathrm{pk}_{D H}$.

Since we have that only two sets of distinct signatures, we consider $p=2, \alpha=\{1\}, \beta=\{2\}$ with $\left(\Sigma_{\alpha}, \mathrm{E}_{\alpha}\right)=$ $\left(\Sigma_{\mathrm{DH}}, \mathrm{E}_{\mathrm{DH}}\right)$ (as defined in Example 1 after renaming as explained above) whereas $\Sigma_{\beta}=\left\{\operatorname{senc}_{D H}\right.$, $\left.\operatorname{sdec}_{D H}\right\}$ and $\mathrm{E}_{\beta}=\left\{\operatorname{sdec}_{D H}\left(\operatorname{senc}_{D H}(x, y), y\right)=x\right\}$. This equational theory is used to model symmetric encryption/decryption, i.e. the primitives used in the processes $Q_{A}$ and $Q_{B}$. Note that when function symbols of $\Sigma_{0}$ are used by only one of the protocols to compose, we can either consider them as part of $\Sigma_{0}$ and so they will be tagged, or they can be put into distinct signatures (using renaming as above) and so they will not be 
tagged. The composition theorem can be applied both ways. Now, we consider $P=P_{A}^{\prime} \mid P_{B}^{\prime}$ where:

$$
\begin{aligned}
& P_{A}^{\prime}=\operatorname{out}\left(c, \operatorname{aenc}_{D H}\left(\mathrm{~g}\left(r_{A}\right), \mathrm{pk}_{D H}\left(s k_{B}\right)\right)\right) \cdot \operatorname{in}\left(c, y_{A}\right) . \\
& {\left[x_{A}:=\mathrm{f}\left(\operatorname{adec}_{D H}\left(y_{A}, s k_{A}\right), r_{A}\right)\right] \cdot \operatorname{out}\left(c, \operatorname{senc}_{D H}\left(s_{A}, x_{A}\right)\right)} \\
& P_{B}^{\prime}=\operatorname{out}\left(c, \operatorname{aenc}_{D H}\left(\mathrm{~g}\left(r_{B}\right), \operatorname{pk}\left(s k_{A}\right)\right)\right) \cdot \operatorname{in}\left(c, y_{B}\right) . \\
& {\left[x_{B}:=\mathrm{f}\left(\operatorname{adec}_{D H}\left(y_{B}, s k_{B}\right), r_{B}\right)\right] \cdot \operatorname{out}\left(c, \operatorname{senc}_{D H}\left(s_{B}, x_{B}\right)\right)}
\end{aligned}
$$

Note that $b n(P)=f v(P)=\emptyset$. We choose to color the three first actions of $P_{A}^{\prime}$ (resp. $P_{B}^{\prime}$ ) with $1 \in \alpha$, and the remaining ones (i.e. those that come from $Q_{A}$ and $Q_{B}$ ) with $2 \in \beta$.

We denote $f n^{\gamma}(P)$ the set of free names of $P$ that occur in actions colored with $\gamma$, and $f v^{\gamma}(P)$ the set of variables of $P$ that occur in an action colored with $\gamma$, and that are not bound by an action colored with $\gamma$. We consider a set $\mathcal{E}_{0}$ of names such that $f n^{\alpha}(P) \cap f n^{\beta}(P) \cap \mathcal{E}_{0}=\emptyset$. This means that each name in $\mathcal{E}_{0}$ can only occur in one type of actions (those colored $\alpha$ or those colored $\beta$ ). We denote $z_{1}^{\alpha}, \ldots, z_{k}^{\alpha}$ (resp. $z_{1}^{\beta}, \ldots, z_{l}^{\beta}$ ) the variables occurring in the left-hand side of an assignment colored $\alpha$ (resp. $\beta$ ), i.e. the variable $x$ such that the action $[x:=v]$ occurs in $P$ and is colored $\alpha$ (resp. $\beta$ ). We assume that $f v^{\alpha}(P) \subseteq\left\{z_{1}^{\beta}, \ldots, z_{l}^{\beta}\right\}$ and $f v^{\beta}(P) \subseteq$ $\left\{z_{1}^{\alpha}, \ldots, z_{k}^{\alpha}\right\}$.

These conditions ensure that sharing between the parts of the process which are colored in different ways is only possible via the assignment variables. This is not a real limitation but this allows us to easily keep track of the shared data.

Example 29: Continuing our previous example, we have that $f n^{\alpha}(P)=\left\{r_{A}, r_{B}, s k_{A}, s k_{B}\right\}$ and $f n^{\beta}(P)=\left\{s_{A}, s_{B}\right\}$. Regarding variables: $f v^{\alpha}(P)=\emptyset$, whereas $f v^{\beta}(P)=$ $\left\{x_{A}, x_{B}\right\}$.

Let $\mathcal{E}_{0}=f n^{\alpha}(P) \cup f n^{\beta}(P)$. To follow the same notation as those introduced in this section, we may want to rename $x_{A}$ with $z_{1}^{\alpha}$ and $x_{B}$ with $z_{2}^{\alpha}$. Note that $f v^{\beta}(P) \subseteq\left\{z_{1}^{\alpha}, z_{2}^{\alpha}\right\}$.

Let $\mathcal{E}_{\alpha}=\left\{n_{1}^{\alpha}, \ldots, n_{k}^{\alpha}\right\}$ and $\mathcal{E}_{\beta}=\left\{n_{1}^{\beta}, \ldots, n_{l}^{\beta}\right\}$ be two sets of fresh names of base type such that $\mathcal{E}_{\alpha} \cap \mathcal{E}_{\beta}=\emptyset$. We define $\rho_{\alpha}$ and $\rho_{\beta}$ as follows:

- $\operatorname{dom}\left(\rho_{\alpha}\right)=\left\{z_{1}^{\beta}, \ldots, z_{l}^{\beta}\right\}, \operatorname{dom}\left(\rho_{\beta}\right)=\left\{z_{1}^{\alpha}, \ldots, z_{k}^{\alpha}\right\}$;

- $\rho_{\alpha}\left(z_{i}^{\beta}\right)=n_{i}^{\beta}$ for each $i \in\{1, \ldots, l\}$; and

- $\rho_{\beta}\left(z_{i}^{\alpha}\right)=n_{i}^{\alpha}$ for each $i \in\{1, \ldots, k\}$.

We do not assume that names in $\mathcal{E}_{\alpha}$ (resp. $\mathcal{E}_{\beta}$ ) are distinct. For instance, we may have $n_{j}^{\alpha}=n_{j^{\prime}}^{\alpha}$ for some $j \neq j^{\prime}$.

Given a colored plain process $P$, we denote by $\delta_{\rho_{\alpha}, \rho_{\beta}}(P)$, the process obtained by applying $\rho_{\alpha}$ on actions colored $\alpha$, and $\rho_{\beta}$ on actions colored $\beta$. This transformation maps the shared case to a particular disjoint case.

Example 30: Let $\mathcal{E}_{\alpha}=\left\{k^{\alpha}\right\}$ and $\mathcal{E}_{\beta}=\emptyset$, and consider the function $\rho_{\beta}$ defined as follows: $\rho_{\beta}\left(z_{1}^{\alpha}\right)=\rho_{\beta}\left(z_{2}^{\alpha}\right)=k^{\alpha}$. Applying $\delta_{\rho_{\alpha}, \rho_{\beta}}$ on $P$ gives us $D_{A} \mid D_{B}$ where:

- $D_{A}=\operatorname{out}\left(c, \operatorname{aenc}\left(\mathrm{g}\left(r_{A}\right), \operatorname{pk}\left(s k_{B}\right)\right)\right)$.in $\left(c, y_{A}\right)$.

$\left[x_{A}:=\mathrm{f}\left(\operatorname{adec}\left(y_{A}, s k_{A}\right), r_{A}\right)\right] . \operatorname{out}\left(c, \operatorname{senc}\left(s_{A}, k^{\alpha}\right)\right)$

- $D_{B}=\operatorname{out}\left(c, \operatorname{aenc}\left(\mathrm{g}\left(r_{B}\right), \operatorname{pk}\left(s k_{A}\right)\right)\right)$.in $\left(c, y_{B}\right)$.

$\left[x_{B}:=\mathrm{f}\left(\operatorname{adec}\left(y_{B}, s k_{B}\right), r_{B}\right)\right] . \operatorname{out}\left(c, \operatorname{senc}\left(s_{B}, k^{\alpha}\right)\right)$
Note that there is no sharing anymore between the part of the process colored $\alpha$ and the part of the process colored $\beta$.

Actually, the disjoint case obtained using the transformation $\delta_{\rho_{\alpha}, \rho_{\beta}}$ behaves as the shared case but only along executions that are compatible with the chosen abstractions, i.e. executions that preserve the equalities and the inequalities among assignment variables as done by the chosen abstraction. This notion is formally defined as follows:

Let $A$ be any extended process derived from $\left(\mathcal{E}_{\alpha} \uplus \mathcal{E}_{\beta} \uplus\right.$ $\left.\mathcal{E}_{0} ; \llbracket P \rrbracket ; \emptyset\right)$, i.e. such that $\left(\mathcal{E}_{\alpha} \uplus \mathcal{E}_{\beta} \uplus \mathcal{E}_{0} ; \llbracket P \rrbracket ; \emptyset\right) \stackrel{\text { tr }}{\Longrightarrow} A$. For $\gamma \in$ $\{\alpha, \beta\}$, we say that $\rho_{\gamma}$ is compatible with $A=(\mathcal{E} ; \mathcal{P} ; \Phi ; \sigma)$ when:

1) for all $x, y \in \operatorname{dom}(\sigma) \cap \operatorname{dom}\left(\rho_{\gamma}\right)$, we have that $x \sigma={ }_{\mathrm{E}}$ $y \sigma$ if, and only if, $x \rho_{\gamma}=y \rho_{\gamma}$; and

2) for all $z \in \operatorname{dom}\left(\rho_{\gamma}\right)$, either $\operatorname{tagroot}(z \sigma \downarrow)=\perp$ or $\operatorname{tag} \operatorname{root}(z \sigma \downarrow) \notin \gamma \cup\{0\}$.

We say that $\left(\rho_{\alpha}, \rho_{\beta}\right)$ is compatible with $A$ when both $\rho_{\alpha}$ and $\rho_{\beta}$ are compatible with $A$.

Before stating our generic composition result, we have also to formalize the fact that the shared keys are not revealed. Since sharing is performed via the assignment variables, we say that $A_{0}$ does not reveal the value of its assignments w.r.t. $\left(\rho_{\alpha}, \rho_{\beta}\right)$ if for any extended process $A=(\mathcal{E} ; \mathcal{P} ; \Phi ; \sigma)$ derived from $A_{0}$ and such that $\left(\rho_{\alpha}, \rho_{\beta}\right)$ is compatible with $A$, we have: new $\mathcal{E} . \Phi \nvdash k$ for any $k \in K_{\alpha} \cup K_{\beta}$

where for all $\gamma \in\{\alpha, \beta\}, K_{\gamma}=\{t, \operatorname{pk}(t), \operatorname{vk}(t) \mid z \in$ $\operatorname{dom}(\sigma) \cap \operatorname{dom}\left(\rho_{\gamma}\right)$ and $\left(t=z \sigma\right.$ or $\left.\left.t=z \rho_{\gamma}\right)\right\}$.

Theorem 31: Let $P$ be a plain colored process as described above, and $B_{0}$ be an extended colored biprocess such that:

- $S_{0}=\left(\mathcal{E}_{\alpha} \uplus \mathcal{E}_{\beta} \uplus \mathcal{E}_{0} ; \llbracket P \rrbracket ; \emptyset ; \emptyset\right) \stackrel{\text { def }}{=} \mathrm{fst}\left(B_{0}\right)$,

- $D_{0}=\left(\mathcal{E}_{\alpha} \uplus \mathcal{E}_{\beta} \uplus \mathcal{E}_{0} ; P_{D} ; \emptyset ; \emptyset\right) \stackrel{\text { def }}{=} \operatorname{snd}\left(B_{0}\right)$, and

- $P_{D}=\delta_{\rho_{\alpha}, \rho_{\beta}}(\llbracket P \rrbracket)$ for some $\left(\rho_{\alpha}, \rho_{\beta}\right)$ compatible with $D_{0}$, and

- $D_{0}$ does not reveal its assignments w.r.t. $\left(\rho_{\alpha}, \rho_{\beta}\right)$.

We have that:

1) For any extended process $S=\left(\mathcal{E}_{S} ; \mathcal{P}_{S} ; \Phi_{S} ; \sigma_{S}\right)$ such that $S_{0} \stackrel{\text { tr }}{\Longrightarrow} S$ with $\left(\rho_{\alpha}, \rho_{\beta}\right)$ compatible with $S$, there exists a biprocess $B$ and an extended process $D=$ $\left(\mathcal{E}_{D} ; \mathcal{P}_{D} ; \Phi_{D} ; \sigma_{D}\right)$ such that $B_{0} \stackrel{\text { tr }}{\Longrightarrow}$ bi $B$, fst $(B)=S$, $\operatorname{snd}(B)=D$, and new $\mathcal{E}_{S} . \Phi_{S} \sim$ new $\mathcal{E}_{D} . \Phi_{D}$.

2) For any extended process $D=\left(\mathcal{E}_{D} ; \mathcal{P}_{D} ; \Phi_{D} ; \sigma_{D}\right)$ such that $D_{0} \stackrel{\operatorname{tr}}{\Longrightarrow} D$ with $\left(\rho_{\alpha}, \rho_{\beta}\right)$ compatible with $D$, there exists a biprocess $B$ and an extended process $S=\left(\mathcal{E}_{S} ; \mathcal{P}_{S} ; \Phi_{S} ; \sigma_{S}\right)$ such that $B_{0} \stackrel{\text { tr }}{\Longrightarrow}$ bi $B$, fst $(B)=S$, $\operatorname{snd}(B)=D$, and new $\mathcal{E}_{S} . \Phi_{S} \sim$ new $\mathcal{E}_{D} . \Phi_{D}$.

This theorem is proved by induction on the length of the derivation. For this, a strong correspondence between the process $S_{0}$ (shared case) and $D_{0}$ (disjoint case) has to be maintained along the derivation, and the transformation $\delta_{\rho_{\alpha}, \rho_{\beta}}$ has to be extended to allow replacements also in $\sigma$ and $\Phi$.

Example 32: Going back to our running example, and forming a biprocess with $S_{0}=\left(\mathcal{E}_{0} \cup\left\{k^{\alpha}\right\} ; P_{A}^{\prime} \mid P_{B}^{\prime} ; \emptyset ; \emptyset\right)$ and 
$D_{0}=\left(\mathcal{E}_{0} \cup\left\{k^{\alpha}\right\} ; D_{A} \mid D_{B} ; \emptyset ; \emptyset\right)$, Theorem 31 gives us that these two processes behave in the same way when considering executions that are compatible with the chosen abstraction $\rho_{\beta}$, i.e. executions that instantiate $x_{A}$ and $x_{B}$ by the same value.

A similar result as the one stated in Theorem 31 was proved in [10]. Here, we consider in addition else branches, and we consider a richer common signature. Moreover, relying on the notion of biprocess, we show a strong link between the shared case and the disjoint case, and we prove in addition static equivalence of the resulting frames.

\section{Name replacement}

Now that we have fixed some notations, we have to explain how the replacement will be applied on the shared process to extract the disjoint case. Actually a same term will be abstracted differently depending on the context which is just above it.

Definition 33: Let $\left(\rho_{\alpha}^{+}, \rho_{\beta}^{+}\right)$be two functions from terms of base type to names of base type. Let $\delta_{\gamma}^{\rho_{\alpha}^{+}, \rho_{\beta}^{+}}$, or shortly $\delta_{\gamma},(\gamma \in\{\alpha, \beta\})$ be the functions on terms that is defined as follows:

$$
\delta_{\gamma}(u)=u \downarrow \rho_{\gamma}^{+} \text {when }\left\{\begin{array}{l}
u \downarrow \in \operatorname{dom}\left(\rho_{\gamma}^{+}\right) \\
\text {and } \operatorname{tagroot}(u) \notin \gamma \cup\{0\}
\end{array}\right.
$$

Otherwise, we have that $\delta_{\gamma}(u)=u$ when $u$ is a name or a variable; and $\delta_{\gamma}\left(f\left(t_{1}, \ldots, t_{k}\right)\right)$ is equal to

- $\mathrm{f}\left(\delta_{\gamma}\left(t_{1}\right), \ldots, \delta_{\gamma}\left(t_{k}\right)\right)$ if tagroot $\left(\mathrm{f}\left(t_{1}, \ldots, t_{n}\right)\right)=0$;

- $\mathrm{f}\left(\delta_{\alpha}\left(t_{1}\right), \ldots, \delta_{\alpha}\left(t_{k}\right)\right)$ if tagroot $\left(\mathrm{f}\left(t_{1}, \ldots, t_{n}\right)\right) \in \alpha$.

- $\mathrm{f}\left(\delta_{\beta}\left(t_{1}\right), \ldots, \delta_{\beta}\left(t_{k}\right)\right)$ if tagroot $\left(\mathrm{f}\left(t_{1}, \ldots, t_{n}\right)\right) \in \beta$.

Definition 34 (Factor for $\Sigma_{0}$ ): Let $u$ be a term. We define Fct $_{\Sigma_{0}}(u)$ the factors of a term $u$ for $\Sigma_{0}$ as the maximal syntactic subterms $v$ of $u$ such that $\operatorname{tag} \operatorname{root}(v) \neq 0$.

Let $\sigma$ be a substitution. We consider a pair $\left(\rho_{\alpha}, \rho_{\beta}\right)$ as defined in Section C-B and compatible with $\sigma$. We denote $\left(\rho_{\alpha}^{+}, \rho_{\beta}^{+}\right)$the extension of $\left(\rho_{\alpha}, \rho_{\beta}\right)$ w.r.t. $\sigma$. Thanks to compatibility, $\rho_{\alpha}^{+}$(resp. $\rho_{\beta}^{+}$) is injective on $\operatorname{dom}\left(\rho_{\alpha}^{+}\right) \backslash\left\{z_{1}^{\beta}, \ldots, z_{l}^{\beta}\right\}$ (resp. $\left.\operatorname{dom}\left(\rho_{\beta}^{+}\right) \backslash\left\{z_{1}^{\alpha}, \ldots, z_{k}^{\alpha}\right\}\right)$. Moreover, we also have that for all $u \in \operatorname{dom}\left(\rho_{\alpha}^{+}\right) \backslash\left\{z_{1}^{\beta}, \ldots, z_{l}^{\beta}\right\}$ (resp. $\operatorname{dom}\left(\rho_{\beta}^{+}\right) \backslash$ $\left.\left\{z_{1}^{\alpha}, \ldots, z_{k}^{\alpha}\right\}\right)$, either tagroot $(u)=\perp$ or $\operatorname{tagroot}(u) \notin \alpha \cup\{0\}$ (resp. $\operatorname{tagroot}(u) \notin \beta \cup\{0\}$ ).

Lemma 35: Let $t_{1}$ and $t_{2}$ be ground terms in normal form such that $\left(f n\left(t_{1}\right) \cup f n\left(t_{2}\right)\right) \cap\left(\mathcal{E}_{\alpha} \uplus \mathcal{E}_{\beta}\right)=\emptyset$. We have that: $t_{1}=t_{2}$ if, and only if, $\delta_{\gamma}\left(t_{1}\right)=\delta_{\gamma}\left(t_{2}\right)$

where $\gamma \in\{\alpha, \beta\}$.

Proof: The right implication is trivial. We consider the left implication, and we prove the result by induction on $\max \left(\left|t_{1}\right|,\left|t_{2}\right|\right)$ when $\gamma=\alpha$. The other case $\gamma=\beta$ can be handled in a similar way.

Base case $\max \left(\left|t_{1}\right|,\left|t_{2}\right|\right)=1$ : In such a case, we have that $t_{1}, t_{2} \in \mathcal{N}$. We first assume that $\delta_{\alpha}\left(t_{1}\right)$ (and thus also $\delta_{\alpha}\left(t_{2}\right)$ ) is in $\mathcal{E}_{\alpha} \uplus \mathcal{E}_{\beta}$. By hypothesis, we know that $t_{2}$ and $t_{1}$ do not use names in $\mathcal{E}_{\alpha} \uplus \mathcal{E}_{\beta}$. Therefore, by definition of $\delta_{\alpha}$, we can deduce that $t_{1}, t_{2} \in \operatorname{dom}\left(\rho_{\alpha}^{+}\right)$and $t_{1} \rho_{\alpha}^{+}=t_{2} \rho_{\alpha}^{+}$, and thus $t_{1}=$ $t_{2}$ thanks to $\rho_{\alpha}^{+}$being injective on $\operatorname{dom}\left(\rho_{\alpha}^{+}\right) \backslash\left\{z_{1}^{\beta}, \ldots, z_{l}^{\beta}\right\}$.
Now, we assume that $\delta_{\alpha}\left(t_{1}\right)$ (and thus also $\delta_{\alpha}\left(t_{2}\right)$ ) is not in $\mathcal{E}_{\alpha} \uplus \mathcal{E}_{\beta}$. In such a case, by definition of $\delta_{\alpha}$, we have that $\delta_{\alpha}\left(t_{1}\right)=t_{1}$ and $\delta_{\alpha}\left(t_{2}\right)=t_{2}$, and thus $t_{1}=t_{2}$.

Inductive step $\max \left(\left|t_{1}\right|,\left|t_{2}\right|\right)>1$ : Assume w.l.o.g. that $\left|t_{1}\right|>$ 1 . Thus, there exists a symbol function $\mathrm{f}$ and terms $u_{1}, \ldots u_{n}$ such that $t_{1}=\mathrm{f}\left(u_{1}, \ldots u_{n}\right)$. We do a case analysis on $t_{1}$ which is in normal form.

Case $t_{1} \in \operatorname{dom}\left(\rho_{\alpha}^{+}\right)$: In such a case, $\delta_{\alpha}\left(t_{1}\right)=\delta_{\alpha}\left(t_{2}\right)=n$ for some $n \in \mathcal{E}_{\alpha}$. By hypothesis, we know that $t_{2}$ and $t_{1}$ do not use names in $\mathcal{E}_{\alpha}$, and we have that $t_{1} \rho_{\alpha}^{+}=t_{2} \rho_{\alpha}^{+}$. Therefore, we necessarily have that $t_{1}=t_{2}$.

Case $t_{1} \notin \operatorname{dom}\left(\rho_{\alpha}^{+}\right)$: We do a new case analysis on $t_{1}$.

Case $f \in \Sigma_{i}^{+}$for some $i \in\{1, \ldots, p\}$ : Let $\gamma \in\{\alpha, \beta\}$ such that $i \in \gamma$. In such a case, we have that $\delta_{\alpha}\left(t_{1}\right)=$ $\mathrm{f}\left(\delta_{\gamma}\left(u_{1}\right), \ldots, \delta_{\gamma}\left(u_{n}\right)\right)$. But $\delta_{\alpha}\left(t_{2}\right)=\delta_{\alpha}\left(t_{1}\right)$ and by definition of $\delta_{\alpha}$, it implies that there exist $v_{1}, \ldots, v_{n}$ such that $t_{2}=$ $\mathrm{f}\left(v_{1}, \ldots, v_{n}\right)$ and $\mathrm{f}\left(\delta_{\gamma}\left(v_{1}\right), \ldots, \delta_{\gamma}\left(v_{n}\right)\right)=\delta_{\alpha}\left(t_{2}\right)$. Thus we have that $\delta_{\gamma}\left(v_{j}\right)=\delta_{\gamma}\left(u_{j}\right)$ for all $j \in\{1, \ldots, n\}$. Furthermore, since $t_{1}$ and $t_{2}$ are in normal form and not using names in $\mathcal{E}_{\alpha} \uplus \mathcal{E}_{\beta}$, we also know that $u_{j}$ and $v_{j}$ are in normal form and not using names in $\mathcal{E}_{\alpha} \uplus \mathcal{E}_{\beta}$, for every $j$. Since, we have that $\max \left(\left|t_{1}\right|,\left|t_{2}\right|\right)>\max \left(\left|u_{j}\right|,\left|v_{j}\right|\right)$, for any $j$, by our inductive hypothesis, we can deduce that $u_{j}=v_{j}$, for all $j$ and so $t_{1}=\mathrm{f}\left(u_{1}, \ldots, u_{n}\right)=\mathrm{f}\left(v_{1}, \ldots, v_{n}\right)=t_{2}$.

Case $t_{1}=\mathrm{f}\left(\operatorname{tag}_{i}\left(w_{1}\right), w_{2}\right)$ with $i \in\{1, \ldots, p\}$ and $\mathrm{f} \in\{$ senc, aenc, sign $\}$ : Let $\gamma \in\{\alpha, \beta\}$ such that $i \in \gamma$. In such a case, we know that $\delta_{\alpha}\left(t_{1}\right)=\mathrm{f}\left(\operatorname{tag}_{i}\left(\delta_{\gamma}\left(w_{1}\right)\right), \delta_{\gamma}\left(w_{2}\right)\right)$. But we know that $\delta_{\alpha}\left(t_{2}\right)=\delta_{\alpha}\left(t_{1}\right)=\mathrm{f}\left(\operatorname{tag}_{i}\left(\delta_{\gamma}\left(w_{1}\right)\right), \delta_{\gamma}\left(w_{2}\right)\right)$. Thus thanks to $t_{2}$ being in normal form and by definition of $\delta_{\alpha}$, it implies that there exists $v_{1}$ and $v_{2}$ such that $t_{2}=\mathrm{f}\left(\operatorname{tag}_{i}\left(v_{1}\right), v_{2}\right)$ and so $\delta_{\alpha}\left(t_{2}\right)=\mathrm{f}\left(\operatorname{tag}_{i}\left(\delta_{\gamma}\left(v_{1}\right)\right), \delta_{\gamma}\left(v_{2}\right)\right)$. Thus, we have that $\delta_{\gamma}\left(v_{1}\right)=\delta_{\gamma}\left(u_{1}\right)$ and $\delta_{\gamma}\left(v_{2}\right)=\delta_{\gamma}\left(u_{2}\right)$. Moreover, $t_{1}$ and $t_{2}$ being in normal form and not using names in $\mathcal{E}_{\alpha} \uplus \mathcal{E}_{\beta}$, so are $u_{j}$ and $v_{j}$ for $j \in\{1,2\}$, so we can apply inductive hypothesis and conclude that $v_{1}=u_{1}$ and $v_{2}=u_{2}$ and so $t_{1}=t_{2}$.

Case $t_{1}=\mathrm{h}\left(\operatorname{tag}_{i}\left(w_{1}\right)\right)$ with $i \in\{1, \ldots, p\}$ : This case is analogous to the previous one.

Case $\mathrm{f} \in \Sigma_{0}$ and $\operatorname{root}\left(u_{1}\right) \neq \operatorname{tag}_{i}, i=1 \ldots p$ : By definition of $\delta_{\alpha}$, we can deduce that $\delta_{\alpha}\left(t_{1}\right)=\mathrm{f}\left(\delta_{\alpha}\left(u_{1}\right), \ldots, \delta_{\alpha}\left(u_{n}\right)\right)$. Since $\delta_{\alpha}\left(t_{1}\right)=\delta_{\alpha}\left(t_{2}\right)$, we can deduce that the top symbol of $t_{2}$ is also $f$ and so there exists $v_{1}, \ldots, v_{n}$ such that $t_{2}=\mathrm{f}\left(v_{1}, \ldots, v_{n}\right)$. In the previous cases, we showed that if $\mathrm{f} \in$ \{senc, aenc, sign, $\mathrm{h}\}$ and the top symbol of $v_{1}$ is $\operatorname{tag}_{j}$ for some $j \in\{1, \ldots, p\}$ then $\delta_{\alpha}\left(t_{1}\right)=\delta_{\alpha}\left(t_{2}\right)$ implies that the top symbol of $u_{1}$ is also $\operatorname{tag}_{j}$. Thus, thanks to our hypothesis, we can deduce that either $\mathrm{f} \notin\{$ senc, aenc, sign, $\mathrm{h}\}$ or the top symbol of $v_{1}$ is different from $\operatorname{tag}_{j}$ for some $j \in\{1, \ldots, p\}$. Hence by definition of $\delta_{\alpha}$, we can deduce that $\delta_{\alpha}\left(t_{2}\right)=\mathrm{f}\left(\delta_{\alpha}\left(v_{1}\right), \ldots, \delta_{\alpha}\left(v_{n}\right)\right)$ and so $\delta_{\alpha}\left(v_{j}\right)=\delta_{\alpha}\left(u_{j}\right)$ for all $j \in\{1, \ldots, n\}$. Moreover, $t_{1}$ and $t_{2}$ being in normal form and not using names in $\mathcal{E}_{\alpha} \uplus \mathcal{E}_{\beta}$, implies that so are $u_{j}$ and $v_{j}$ for all $j \in\{1, \ldots, n\}$. We can thus apply our inductive hypothesis and conclude that $u_{j}=v_{j}$ for all $j \in\{1, \ldots, n\}$ and so $t_{1}=t_{2}$. 
Lemma 36: Let $t_{1}$ and $t_{2}$ be ground terms in normal form such that $\left(f n\left(t_{1}\right) \cup f n\left(t_{2}\right)\right) \cap\left(\mathcal{E}_{\alpha} \uplus \mathcal{E}_{\beta}\right)=\emptyset$. We have that: $\delta_{\alpha}\left(t_{1}\right)=\delta_{\beta}\left(t_{2}\right)$ implies that $t_{1}=t_{2}$.

Proof: We prove the result by induction on $\left|\delta_{\alpha}\left(t_{1}\right)\right|$.

Base case $\left|\delta_{\alpha}\left(t_{1}\right)\right|=1$ : Since $\delta_{\alpha}\left(t_{1}\right)=\delta_{\beta}\left(t_{2}\right), \mathcal{E}_{\alpha} \cap \mathcal{E}_{\beta}=\emptyset$, and $t_{1}, t_{2}$ do not use names in $\mathcal{E}_{\alpha} \uplus \mathcal{E}_{\beta}$, we necessarily have that $t_{1} \notin \operatorname{dom}\left(\rho_{\alpha}^{+}\right)$and $t_{2} \notin \operatorname{dom}\left(\rho_{\beta}^{+}\right)$. Hence, we have that $\delta_{\alpha}\left(t_{1}\right)=t_{1}$ and $\delta_{\beta}\left(t_{2}\right)=t_{2}$. This allows us to conclude.

Inductive step $\left|\delta_{\alpha}\left(t_{1}\right)\right|>1$ : In that case, we have that $\delta_{\alpha}\left(t_{1}\right)=\mathrm{f}\left(u_{1}, \ldots, u_{n}\right)=\delta_{\beta}\left(t_{2}\right)$. Assume that $\mathrm{f} \in \Sigma_{i} \cup$ $\Sigma_{\operatorname{tag}_{i}}$ for some $i \in\{1, \ldots, p\}$. Let $\gamma \in\{\alpha, \beta\}$ such that $i \in \gamma$. By definition of $\delta_{\alpha}$ and $\delta_{\beta}$, we can deduce that $\operatorname{root}\left(t_{1}\right)=\mathrm{f}=\operatorname{root}\left(t_{2}\right)$. Furthermore, if we assume that $t_{1}=\mathrm{f}\left(v_{1}, \ldots, v_{n}\right)$ and $t_{2}=\mathrm{f}\left(w_{1}, \ldots, w_{n}\right)$, we would have $\delta_{\gamma}\left(v_{j}\right)=\delta_{\gamma}\left(w_{j}\right)$ for all $j \in\{1, \ldots, n\}$. By Lemma 35, we deduce that $v_{j}=w_{j}$ for all $j \in\{1, \ldots, n\}$. Hence, we conclude that $t_{1}=t_{2}$. Assume now that $\mathrm{f} \in \Sigma_{0}$. According to the definition of $\delta_{\alpha}$ and $\delta_{\beta}$, there exists $v_{1}, \ldots, v_{n}$ and $w_{1}, \ldots, w_{n}$ such that $t_{1}=\mathrm{f}\left(v_{1}, \ldots, v_{n}\right), t_{2}=\mathrm{f}\left(w_{1}, \ldots, w_{n}\right)$ and $\delta_{\gamma_{1}}\left(v_{j}\right)=\delta_{\gamma_{2}}\left(w_{j}\right)$, for some $\gamma_{1}, \gamma_{2} \in\{\alpha, \beta\}$. Moreover, $t_{1}$ and $t_{2}$ being in normal form and not using names in $\mathcal{E}_{\alpha} \uplus \mathcal{E}_{\beta}$ implies that so are $v_{j}$ and $w_{j}$ for all $j \in\{1, \ldots, n\}$. Now, either $\gamma_{1}=\gamma_{2}$ and so by Lemma 35, we have that $v_{j}=w_{j}$, else $\gamma_{1} \neq \gamma_{2}$ but then by our inductive hypothesis, we also have $v_{j}=w_{j}$. Hence we conclude that $t_{1}=t_{2}$.

Lemma 37: Let $u$ be a ground term in normal form such that $f n(u) \cap\left(\mathcal{E}_{\alpha} \uplus \mathcal{E}_{\beta}\right)=\emptyset$. Let $\gamma \in\{\alpha, \beta\}$. We have that:

- $\delta_{\gamma}(u)$ is in normal form; and

- either $\operatorname{root}\left(\delta_{\gamma}(u)\right)=\operatorname{root}(u)$ or $\operatorname{root}\left(\delta_{\gamma}(u)\right)=\perp$.

- either $\operatorname{tagroot}\left(\delta_{\gamma}(u)\right)=\operatorname{tagroot}(u)$ or tagroot $\left(\delta_{\gamma}(u)\right)=$ $\perp$.

Proof: We prove this result by induction on $|u|$ and we assume w.l.o.g. that $\gamma=\alpha$.

Base case $|u|=1$ : In such a case, we have that $u \in \mathcal{N}$, and we also have that $\delta_{\alpha}(u) \in \mathcal{N}$ and so $\delta_{\alpha}(u)$ is in normal form with the same root as $u$, namely $\perp$. Moreover, we have $\operatorname{tagroot}\left(\delta_{\gamma}(u)\right)=\perp$.

Inductive $|u|>1$ : Assume first that $u \downarrow \in \operatorname{dom}\left(\rho_{\alpha}^{+}\right)$and $\operatorname{tagroot}(u) \notin \alpha \cup\{0\}$. Hence by definition of $\delta_{\alpha}$, we have that $\delta_{\alpha}(u) \in \mathcal{E}_{\alpha}$. Thus, we trivially obtain that $\delta_{\alpha}(u)$ is in normal form, $\operatorname{root}\left(\delta_{\alpha}(u)\right)=\perp$ and $\operatorname{tagroot}\left(\delta_{\alpha}(u)\right)=\perp$.

Otherwise, we distinguish two cases:

Case 1. We have that $u=C\left[u_{1}, \ldots, u_{n}\right]$ where $C$ is built on $\frac{C a s e l}{\Sigma_{j} \cup \Sigma_{\operatorname{tag}_{j}}}$ with $j \in\{1, \ldots, p\}, C$ is different from a hole, $u_{k}$ are factors in normal form of $u, k=1 \ldots n$. Let $\varepsilon \in\{\alpha, \beta\}$ such that $j \in \varepsilon$. Hence, since $u \notin \operatorname{dom}\left(\rho_{\alpha}^{+}\right)$, then by definition of $\delta_{\alpha}$, we deduce that $\delta_{\alpha}(u)=C\left[\delta_{\varepsilon}\left(u_{1}\right), \ldots, \delta_{\varepsilon}\left(u_{n}\right)\right]$. Since $C$ is not a hole, thanks to our inductive hypothesis on $u_{1}, \ldots, u_{n}$, we have that $\delta_{\varepsilon}\left(u_{1}\right), \ldots, \delta_{\varepsilon}\left(u_{n}\right)$ are in normal form and $\delta_{\varepsilon}\left(u_{1}\right), \ldots, \delta_{\varepsilon}\left(u_{n}\right)$ are factors of $\delta_{\alpha}(u)$. Thus, since $u$ is in normal form, we have that $C\left[u_{1}, \ldots, u_{n}\right] \downarrow=C\left[u_{1}, \ldots, u_{n}\right]$. By Lemmas 35 and 27, we deduce that

$$
C\left[\delta_{\varepsilon}\left(u_{1}\right), \ldots, \delta_{\varepsilon}\left(u_{n}\right)\right] \downarrow=C\left[\delta_{\varepsilon}\left(u_{1}\right), \ldots, \delta_{\varepsilon}\left(u_{n}\right)\right]
$$

i.e. $\delta_{\alpha}(u) \downarrow=\delta_{\alpha}(u)$.

Furthermore, we also have that $\operatorname{root}\left(\delta_{\alpha}(u)\right)=\operatorname{root}(u)$ and $\operatorname{tagroot}\left(\delta_{\alpha}(u)\right)=\operatorname{tagroot}(u)$.

Case 2. We have that $u=\mathrm{f}\left(v_{1}, \ldots, v_{n}\right)$ for some $\mathrm{f} \in \Sigma_{0}$. By definition of $\delta_{\alpha}$ there exists $\varepsilon \in\{\alpha, \beta\}$ such that $\delta_{\alpha}(u)=$ $\mathrm{f}\left(\delta_{\varepsilon}\left(v_{1}\right), \ldots, \delta_{\varepsilon}\left(v_{m}\right)\right)$. We do a case analysis on $\mathrm{f}$ :

Case $\mathrm{f} \in\{$ senc, aenc, pk, sign, vk, h, \langle\rangle$\}$ : In this case, we have that $\delta_{\alpha}(u) \downarrow=\mathrm{f}\left(\delta_{\varepsilon}\left(v_{1}\right) \downarrow, \ldots, \delta_{\varepsilon}\left(v_{m}\right) \downarrow\right)$. Since by inductive hypothesis, $\delta_{\varepsilon}\left(v_{k}\right)$ is in normal form, for all $k \in$ $\{1, \ldots, m\}$, we can deduce that $\delta_{\alpha}(u)$ is also in normal form and $\operatorname{root}\left(\delta_{\alpha}(u)\right)=\mathrm{f}=\operatorname{root}(u)$. If $\mathrm{f} \in\{\mathrm{pk}, \mathrm{vk},\langle\rangle\}$ then we trivially have that $\operatorname{tagroot}\left(\delta_{\alpha}(u)\right)=\operatorname{tagroot}(u)$. Let's focus on $\mathrm{f} \in\{$ senc, aenc, sign $\}$. If $\operatorname{tagroot}(u) \notin\{0\}$ then it means that $\operatorname{root}\left(v_{1}\right)=\operatorname{tag}_{i}$ for some $i \in \varepsilon$. But by definition of $\delta_{\varepsilon}$, we would have that $\operatorname{root}\left(\delta_{\varepsilon}\left(v_{1}\right)\right)=\operatorname{tag}_{i}$. Hence $\operatorname{tagroot}\left(\delta_{\alpha}(u)\right)=\operatorname{tagroot}(u)$. Now if $\operatorname{tagroot}(u) \notin\{0\}$, it means that $\operatorname{root}\left(v_{1}\right) /\left\{\operatorname{tag}_{1} \ldots \operatorname{tag}_{p}\right\}$. But by inductive hypothesis, $\operatorname{root}\left(\delta_{\varepsilon}\left(v_{1}\right)\right)=\perp$ or $\operatorname{root}\left(\delta_{\varepsilon}\left(v_{1}\right)\right)=\operatorname{root}\left(v_{1}\right)$ and so we can conclude that tagroot $\left(\delta_{\alpha}(u)\right) \in\{0\}$.

Case $\mathrm{f}=$ sdec: Then $m=2$, and by definition of $\delta_{\alpha}$, we have that $\delta_{\alpha}(u)=\operatorname{sdec}\left(\delta_{\alpha}\left(v_{1}\right), \delta_{\alpha}\left(v_{2}\right)\right)$. Thus, in such a case, we have that $\operatorname{root}\left(\delta_{\alpha}(u)\right)=\mathrm{f}=\operatorname{root}(u)$. By inductive hypothesis, we have that $\delta_{\alpha}\left(v_{1}\right)$ and $\delta_{\alpha}\left(v_{2}\right)$ are both in normal form. Assume that sdec cannot be reduced, i.e. $\delta_{\alpha}(u) \downarrow=\operatorname{sdec}\left(\delta_{\alpha}\left(v_{1}\right) \downarrow, \delta_{\alpha}\left(v_{2}\right) \downarrow\right)=\operatorname{sdec}\left(\delta_{\alpha}\left(v_{1}\right), \delta_{\alpha}\left(v_{2}\right)\right)$. Thus the result holds. Otherwise, if sdec can be reduced, there exist $w_{1}, w_{2}$ with $\delta_{\alpha}\left(v_{1}\right)=\operatorname{senc}\left(w_{1}, w_{2}\right)$ and $\delta_{\alpha}\left(v_{2}\right)=w_{2}$. By definition of $\delta_{\alpha}$, there must exist $\varepsilon^{\prime} \in\{\alpha, \beta\}$, and $w_{1}^{\prime}, w_{2}^{\prime}$ such that $\delta_{\alpha}\left(v_{1}\right)=\operatorname{senc}\left(\delta_{\varepsilon^{\prime}}\left(w_{1}^{\prime}\right), \delta_{\varepsilon^{\prime}}\left(w_{2}^{\prime}\right)\right), v_{1}=$ $\operatorname{senc}\left(w_{1}^{\prime}, w_{2}^{\prime}\right), w_{1}=\delta_{\varepsilon^{\prime}}\left(w_{1}^{\prime}\right)$ and $w_{2}=\delta_{\varepsilon^{\prime}}\left(w_{2}^{\prime}\right)$. Thus, we have that $\delta_{\alpha}\left(v_{2}\right)=\delta \varepsilon^{\prime}\left(w_{2}^{\prime}\right)$. Thanks to Lemmas 35 and 36, we have that $v_{2}=w_{2}^{\prime}$. Hence, $u=\operatorname{sdec}\left(\operatorname{senc}\left(w_{1}^{\prime}, w_{2}^{\prime}\right), w_{2}^{\prime}\right)$. But in such a case, we would have that $u$ is not in normal form which contradicts our hypothesis.

At last, since $\operatorname{root}\left(\delta_{\alpha}(u)\right)=\perp$ or $\operatorname{root}\left(\delta_{\alpha}(u)\right)=\operatorname{root}(u)=$ sdec then we can deduce that $\operatorname{tagroot}\left(\delta_{\alpha}(u)\right)=\perp$ or $\operatorname{tagroot}\left(\delta_{\alpha}(u)\right)=\operatorname{tagroot}(u)=0$.

The cases where $f=$ check or $f=$ adec are analogous to the previous one.

\section{D. $\delta_{\alpha}$ and $\delta_{\beta}$ on tagged term}

Let $\sigma_{0}$ be a ground substitution. Similarly to the previous section, we consider a pair $\left(\rho_{\alpha}, \rho_{\beta}\right)$ as defined in Section C-B and compatible with $\sigma_{0}$. We denote $\left(\rho_{\alpha}^{+}, \rho_{\beta}^{+}\right)$the extension of $\left(\rho_{\alpha}, \rho_{\beta}\right)$ w.r.t. this substitution. We also denote by $\mathcal{E}_{\alpha}$ and $\mathcal{E}_{\beta}$ the respective image of $\rho_{\beta}$ and $\rho_{\alpha}$, and we assume that $\sigma_{0}$ does not use any name in $\mathcal{E}_{\alpha}$ and $\mathcal{E}_{\beta}$.

Thanks to compatibility, $\rho_{\alpha}^{+}$(resp. $\rho_{\beta}^{+}$) is injective on $\operatorname{dom}\left(\rho_{\alpha}^{+}\right) \backslash\left\{z_{1}^{\beta}, \ldots, z_{l}^{\beta}\right\}$ (resp. $\left.\operatorname{dom}\left(\rho_{\beta}^{+}\right) \backslash\left\{z_{1}^{\alpha}, \ldots, z_{k}^{\alpha}\right\}\right)$. Moreover, we also have that for all $z \in\left\{z_{1}^{\beta}, \ldots, z_{l}^{\beta}\right\}$ (resp. $z \in\left\{z_{1}^{\alpha}, \ldots, z_{k}^{\alpha}\right\}$ ), either tagroot $\left(z \sigma_{0} \downarrow\right)=\perp$ or $\operatorname{tagroot}\left(z \sigma_{0} \downarrow\right) \notin \alpha \cup\{0\}$ (resp. tagroot $\left(z \sigma_{0} \downarrow\right) \notin \beta \cup\{0\}$ ).

Let $i \in\{1, \ldots, p\}$. Let $u \in \mathcal{T}\left(\Sigma_{i} \cup \Sigma_{\operatorname{tag}_{i}} \cup \Sigma_{0}, \mathcal{N} \cup \mathcal{X}\right)$. As defined in Section A, test $i_{i}(u)$ is a conjunction of elementary 
formulas (equalities between terms). Given a substitution $\sigma$ such that $f v(u) \subseteq \operatorname{dom}(\sigma)$, we say that $\sigma$ satisfies $t_{1}=t_{2}$, denoted $\sigma \vDash t_{1}=t_{2}$, if $t_{1} \sigma \downarrow=t_{2} \sigma \downarrow$.

At last, for all substitution $\sigma$, for all $\gamma \in\{\alpha, \beta\}$, we denote by $\delta_{\gamma}(\sigma)$ the substitution such that $\operatorname{dom}(\sigma)=\operatorname{dom}\left(\delta_{\gamma}(\sigma)\right)$ and for all $x \in \operatorname{dom}\left(\delta_{\gamma}(\sigma)\right), x \delta_{\gamma}(\sigma)=\delta_{\gamma}(x \sigma)$.

Lemma 38: Let $u \in \mathcal{T}\left(\Sigma_{i} \cup \Sigma_{0}, \mathcal{N} \cup \mathcal{X}\right)$ for some $i \in$ $\{1, \ldots, p\}$. Let $\gamma \in\{\alpha, \beta\}$ such that $i \in \gamma$ and $\sigma_{0}$ be a ground substitution such that $f v(u) \subseteq \operatorname{dom}\left(\sigma_{0}\right)$. Moreover, assume that $u$ does not use names in $\mathcal{E}_{\alpha} \uplus \mathcal{E}_{\beta}$. We have that:

- $\delta_{\gamma}\left([u]_{i}\left(\sigma_{0} \downarrow\right)\right)=\delta_{\gamma}\left([u]_{i}\right) \delta_{\gamma}\left(\sigma_{0} \downarrow\right)$; and

- If $\sigma_{0} \vDash \operatorname{test}_{i}\left([u]_{i}\right)$ then $\delta_{\gamma}\left([u]_{i}\left(\sigma_{0} \downarrow\right)\right) \downarrow=\delta_{\gamma}\left([u]_{i} \sigma_{0} \downarrow\right)$.

Proof: Let $\sigma$ be the substitution $\sigma_{0} \downarrow$. We prove the two results separately. First, we show by induction on $|u|$ that $\delta_{\gamma}\left([u]_{i} \sigma\right)=\delta_{\gamma}\left([u]_{i}\right) \delta_{\gamma}(\sigma)$ :

Base case $|u|=1$ : In this case, $u \in \mathcal{N} \cup \mathcal{X}$. If $u \in \mathcal{N}$ then we have that $[u]_{i}=u$ and so $[u]_{i} \sigma=u$ and $\delta_{\gamma}(u) \in$ $\mathcal{N}$. Thus, we have that $\delta_{\gamma}\left([u]_{i} \sigma\right)=\delta_{\gamma}(u)=\delta_{\gamma}(u) \delta_{\gamma}(\sigma)=$ $\delta_{\gamma}\left([u]_{i}\right) \delta_{\gamma}(\sigma)$. Otherwise, we have that $u \in \mathcal{X}$ and $[u]_{i}=u$. W.l.o.g., we assume that $\gamma=\alpha$. First, if $u \notin\left\{z_{1}^{\beta}, \ldots, z_{l}^{\beta}\right\}$, then we have that $\delta_{\alpha}(u)=u$. Thus, $\delta_{\alpha}(u) \delta_{\alpha}(\sigma)=u \delta_{\alpha}(\sigma)$. Since $u \in \mathcal{X}$ and $f v(u) \subseteq \operatorname{dom}(\sigma)$, we have that $u \delta_{\alpha}(\sigma)=$ $\delta_{\alpha}(u \sigma)$, thus $\delta_{\alpha}\left([u]_{i} \sigma\right)=\delta_{\alpha}(u \sigma)=u \delta_{\alpha}(\sigma)=\delta_{\alpha}(u) \delta_{\alpha}(\sigma)=$ $\delta_{\alpha}\left([u]_{i}\right) \delta_{\alpha}(\sigma)$. Now, it remains the case where $u=z_{j}^{\beta}$ for some $j \in\{1, \ldots, l\}$. In such a case, we have that:

- $\delta_{\alpha}\left(\left[z_{j}^{\beta}\right]_{i} \sigma\right)=\delta_{\alpha}\left(z_{j}^{\beta} \sigma\right)=n_{j}^{\beta}$, and

- $\delta_{\alpha}\left(\left[z_{j}^{\beta}\right]_{i}\right) \delta_{\alpha}(\sigma)=\delta_{\alpha}\left(z_{j}^{\beta}\right) \delta_{\alpha}(\sigma)=n_{j}^{\beta} \delta_{\alpha}(\sigma)=n_{j}^{\beta}$.

Inductive step $|u|>1 \mid$, i.e. $u=\mathrm{f}\left(u_{1}, \ldots, u_{n}\right)$. We do a case analysis on $\mathrm{f}$.

Case $\mathrm{f} \in \Sigma_{i}$ : In such a case, $[u]_{i}=\mathrm{f}\left(\left[u_{1}\right]_{i}, \ldots,\left[u_{n}\right]_{i}\right)$. By definition of $\delta_{\gamma}, \delta_{\gamma}\left([u]_{i} \sigma\right)=\mathrm{f}\left(\delta_{\gamma}\left(\left[u_{1}\right]_{i} \sigma\right), \ldots, \delta_{\gamma}\left(\left[u_{n}\right]_{i} \sigma\right)\right)$ and $\delta_{\gamma}\left([u]_{i}\right)=\mathrm{f}\left(\delta_{\gamma}\left(\left[u_{1}\right]_{i}\right), \ldots, \delta_{\gamma}\left(\left[u_{n}\right]_{i}\right)\right)$. By our inductive hypothesis, we can deduce that for all $k \in\{1, \ldots, n\}$, we have that $\delta_{\gamma}\left(\left[u_{k}\right]_{i} \sigma\right)=\delta_{\gamma}\left(\left[u_{k}\right]_{i}\right) \delta_{\gamma}(\sigma)$. Thus, we can deduce that $\delta_{\gamma}\left([u]_{i} \sigma\right)=\mathrm{f}\left(\delta_{\gamma}\left(\left[u_{1}\right]_{i}\right), \ldots, \delta_{\gamma}\left(\left[u_{n}\right]_{i}\right)\right) \delta_{\gamma}(\sigma)=$ $\delta_{\gamma}\left([u]_{i}\right) \delta_{\gamma}(\sigma)$.

Case $f \in\{$ aenc, sign, senc $\}$ : In this case $n=2$, and by definition of $[u]_{i}$, we have that $[u]_{i}=\mathrm{f}\left(\operatorname{tag}_{i}\left(\left[u_{1}\right]_{i}\right),\left[u_{2}\right]_{i}\right)$. Thus, we have that $\delta_{\gamma}\left([u]_{i}\right)=\mathrm{f}\left(\operatorname{tag}_{i}\left(\delta_{\gamma}\left(\left[u_{1}\right]_{i}\right)\right), \delta_{\gamma}\left(\left[u_{2}\right]_{i}\right)\right)$ and $\delta_{\gamma}\left([u]_{i} \sigma\right)=\mathrm{f}\left(\operatorname{tag}_{i}\left(\delta_{\gamma}\left(\left[u_{1}\right]_{i} \sigma\right)\right), \delta_{\gamma}\left(\left[u_{2}\right]_{i} \sigma\right)\right)$. But by our inductive hypothesis, we have $\delta_{\gamma}\left(\left[u_{k}\right]_{i} \sigma\right)=\delta_{\gamma}\left(\left[u_{k}\right]_{i}\right) \delta_{\gamma}(\sigma)$ with $k \in\{1,2\}$. We conclude that

$$
\begin{aligned}
\delta_{\gamma}\left([u]_{i} \sigma\right) & =\mathrm{f}\left(\operatorname{tag}_{i}\left(\delta_{\gamma}\left(\left[u_{1}\right]_{i}\right) \delta_{\gamma}(\sigma)\right), \delta_{\gamma}\left(\left[u_{2}\right]_{i}\right) \delta_{\gamma}(\sigma)\right) \\
& =\delta_{\gamma}\left([u]_{i}\right) \delta_{\gamma}(\sigma) .
\end{aligned}
$$

Case $\mathrm{f}=\mathrm{h}$ : This case is analogous to the previous one and can be handled in a similar way.

Case $\mathrm{f} \in\{\mathrm{sdec}$, adec, check $\}$ : In this case $n=2$, and by definition of $[u]_{i}$, we have that $[u]_{i}=\operatorname{untag}_{i}\left(\mathrm{f}\left(\left[u_{1}\right]_{i},\left[u_{2}\right]_{i}\right)\right)$. Thus, we have that $\delta_{\gamma}\left([u]_{i}\right)=\operatorname{untag}_{i}\left(f\left(\delta_{\gamma}\left(\left[u_{1}\right]_{i}\right), \delta_{\gamma}\left(\left[u_{2}\right]_{i}\right)\right)\right)$ and $\delta_{\gamma}\left([u]_{i} \sigma\right)=\operatorname{untag}_{i}\left(f\left(\delta_{\gamma}\left(\left[u_{1}\right]_{i} \sigma\right), \delta_{\gamma}\left(\left[u_{2}\right]_{i} \sigma\right)\right)\right)$. Relying on our inductive hypothesis, we deduce that

$$
\delta_{\gamma}\left(\left[u_{k}\right]_{i} \sigma\right)=\delta_{\gamma}\left(\left[u_{k}\right]_{i}\right) \delta_{\gamma}(\sigma) \text { with } k \in\{1,2\} .
$$

We conclude that

$$
\begin{aligned}
\delta_{\gamma}\left([u]_{i} \sigma\right) & =\operatorname{untag}_{i}\left(\mathrm{f}\left(\delta_{\gamma}\left(\left[u_{1}\right]_{i}\right), \delta_{\gamma}\left(\left[u_{2}\right]_{i}\right)\right)\right) \delta_{\gamma}(\sigma) \\
& =\delta_{\gamma}\left([u]_{i}\right) \delta_{\gamma}(\sigma) .
\end{aligned}
$$

Otherwise, by definition of $[u]_{i}$, we have that:

- $[u]_{i}=\mathrm{f}\left(\left[u_{1}\right]_{i}, \ldots,\left[u_{n}\right]_{i}\right)$, and

- $\delta_{\gamma}\left([u]_{i}\right)=\mathrm{f}\left(\delta_{\gamma}\left(\left[u_{1}\right]_{i}\right), \ldots, \delta_{\gamma}\left(\left[u_{n}\right]_{i}\right)\right)$.

Thus, this case is similar to the case $f \in \Sigma_{i}$. Hence the result holds.

We now prove the second property, i.e. if $\sigma \vDash \operatorname{test}_{i}\left([u]_{i}\right)$, then $\delta_{\gamma}\left([u]_{i} \sigma\right) \downarrow=\delta_{\gamma}\left([u]_{i} \sigma \downarrow\right)$. We prove the result by induction on $|u|$ :

Base case $|u|=1$ : In this case, $u \in \mathcal{N} \cup \mathcal{X}$. In both cases, we have that $[u]_{i}=u$ and $\operatorname{test}_{i}(u)=$ true. If $u \in \mathcal{N}$, we know that $\delta_{\gamma}(u) \in \mathcal{N}$ and so $\delta_{\gamma}(u) \downarrow=\delta_{\gamma}(u)$. We also have that $u \sigma \downarrow=u \sigma=u$. This allows us to conclude that

$$
\delta_{\gamma}(u \sigma) \downarrow=\delta_{\gamma}(u) \downarrow=\delta_{\gamma}(u)=\delta_{\gamma}(u \sigma \downarrow) .
$$

Otherwise, we have $u \in \mathcal{X}$. Since $\sigma$ is is normal form, we deduce that $u \sigma \downarrow=u \sigma$. By Lemma 37, we also know that $\delta_{\gamma}(u \sigma \downarrow) \downarrow=\delta_{\gamma}(u \sigma \downarrow)$. Thus, we conclude that

$$
\delta_{\gamma}(u \sigma \downarrow)=\delta_{\gamma}(u \sigma \downarrow) \downarrow=\delta_{\gamma}(u \sigma) \downarrow .
$$

Inductive step $|u|>1$, i.e. $u=\mathrm{f}\left(u_{1}, \ldots, u_{n}\right)$. We do a case analysis on $\mathrm{f}$.

Case $\mathrm{f} \in \Sigma_{i}$ : We have that $[u]_{i}=\mathrm{f}\left(\left[u_{1}\right]_{i}, \ldots,\left[u_{n}\right]_{i}\right)$. Hence, we have that $\delta_{\gamma}\left([u]_{i} \sigma\right)=\mathrm{f}\left(\delta_{\gamma}\left(\left[u_{1}\right]_{i} \sigma\right), \ldots, \delta_{\gamma}\left(\left[u_{n}\right]_{i} \sigma\right)\right)$ and so $\delta_{\gamma}\left([u]_{i} \sigma\right) \downarrow=\mathrm{f}\left(\delta_{\gamma}\left(\left[u_{1}\right]_{i} \sigma\right) \downarrow, \ldots, \delta_{\gamma}\left(\left[u_{n}\right]_{i} \sigma\right) \downarrow\right) \downarrow$. We have that $\operatorname{test}_{i}\left([u]_{i}\right)=\bigwedge_{j=1}^{n} \operatorname{test}_{i}\left(\left[u_{j}\right]_{i}\right)$ which means that $\sigma \vDash$ test $_{i}\left(\left[u_{j}\right]_{i}\right)$ for each $j \in\{1, \ldots, n\}$. By applying our inductive hypothesis on $u_{1}, \ldots, u_{n}$, we deduce that

$$
\begin{aligned}
\delta_{\gamma}\left([u]_{i} \sigma\right) \downarrow & =\mathrm{f}\left(\delta_{\gamma}\left(\left[u_{1}\right]_{i} \sigma \downarrow\right), \ldots, \delta_{\gamma}\left(\left[u_{n}\right]_{i} \sigma \downarrow\right)\right) \downarrow \\
& =\delta_{\gamma}\left(\mathrm{f}\left(\left[u_{1}\right]_{i} \sigma \downarrow, \ldots,\left[u_{n}\right]_{i} \sigma \downarrow\right)\right) \downarrow
\end{aligned}
$$

Let $t=\mathrm{f}\left(\left[u_{1}\right]_{i} \sigma \downarrow, \ldots,\left[u_{n}\right]_{i} \sigma \downarrow\right)$. We can assume that there exists a context $C$ built on $\Sigma_{i}$ such that $t=C\left[t_{1}, \ldots, t_{m}\right]$ with $F c t(t)=\left\{t_{1}, \ldots, t_{m}\right\}$ and $t_{1}, \ldots, t_{m}$ are in normal form. Thus, by Lemma 26, there exists a context $D$ (possibly a hole) such that $t \downarrow=D\left[t_{j_{1}}, \ldots, t_{j_{k}}\right]$ with $j_{1}, \ldots, j_{k} \in\{0, \ldots, m\}$ and $t_{0}=n_{\min }$. Since $t_{1}, \ldots, t_{m}$ are in normal form and thanks to Lemma 37, we know that for all $k \in\{0, \ldots, m\}$, $\delta_{\gamma}\left(t_{k}\right)$ is also in normal form and its root is not in $\Sigma_{i}$. Hence, we can apply Lemma 27 such that $C\left[\delta_{\gamma}\left(t_{1}\right), \ldots, \delta_{\gamma}\left(t_{m}\right)\right] \downarrow=$ $D\left[\delta_{\gamma}\left(t_{j_{1}}\right), \ldots, \delta_{\gamma}\left(t_{j_{k}}\right)\right]$. But since $C$ and $D$ are both built upon $\Sigma_{i}$, we have that:

- $C\left[\delta_{\gamma}\left(t_{1}\right), \ldots, \delta_{\gamma}\left(t_{m}\right)\right] \downarrow=\delta_{\gamma}\left(C\left[t_{1}, \ldots, t_{m}\right]\right) \downarrow$, and

- $D\left[\delta_{\gamma}\left(t_{j_{1}}\right), \ldots, \delta_{\gamma}\left(t_{j_{k}}\right)\right]=\delta_{\gamma}\left(D\left[t_{j_{1}}, \ldots, t_{j_{k}}\right]\right)$.

Hence, we can deduce that $\delta_{\gamma}(t) \downarrow=\delta_{\gamma}(t \downarrow)$. But we already know that $t \downarrow=[u]_{i} \sigma \downarrow$ and $\delta_{\gamma}(t) \downarrow=\delta_{\gamma}\left([u]_{i} \sigma\right) \downarrow$. Thus, we can conclude that $\delta_{\gamma}\left([u]_{i} \sigma\right) \downarrow=\delta_{\gamma}\left([u]_{i} \sigma \downarrow\right)$.

Case $f \in\{$ senc, aenc, sign $\}$ : In such a case, we have that:

- $[u]_{i}=\mathrm{f}\left(\operatorname{tag}_{i}\left(\left[u_{1}\right]_{i}\right),\left[u_{2}\right]_{i}\right)$, and 
- $\operatorname{test}_{i}\left([u]_{i}\right)=\operatorname{test}_{i}\left(\left[u_{1}\right]_{i}\right) \wedge \operatorname{test}_{i}\left(\left[u_{2}\right]_{i}\right)$.

Hence, we have that $[u]_{i} \sigma \downarrow=\mathrm{f}\left(\operatorname{tag}_{i}\left(\left[u_{1}\right]_{i} \sigma \downarrow\right),\left[u_{2}\right]_{i} \sigma \downarrow\right)$, and also $\delta_{\gamma}\left([u]_{i} \sigma\right) \downarrow=\mathrm{f}\left(\operatorname{tag}_{i}\left(\delta_{\gamma}\left(\left[u_{1}\right]_{i} \sigma\right) \downarrow\right), \delta_{\gamma}\left(\left[u_{2}\right]_{i} \sigma\right) \downarrow\right)$. By our inductive hypothesis on $u_{1}$ and $u_{2}$, we have that:

$$
\delta_{\gamma}\left(\left[u_{k}\right]_{i} \sigma\right) \downarrow=\delta_{\gamma}\left(\left[u_{k}\right]_{i} \sigma \downarrow\right) \text { with } k \in\{1,2\} .
$$

Hence, we can deduce that

$$
\begin{aligned}
\delta_{\gamma}\left([u]_{i} \sigma\right) \downarrow & =\mathrm{f}\left(\operatorname{tag}_{i}\left(\delta_{\gamma}\left(\left[u_{1}\right]_{i} \sigma \downarrow\right)\right), \delta_{\gamma}\left(\left[u_{2}\right]_{i} \sigma \downarrow\right)\right) \\
& =\delta_{\gamma}\left(\mathrm{f}\left(\operatorname{tag}_{i}\left(\left[u_{1}\right]_{i} \sigma \downarrow\right),\left[u_{2}\right]_{i} \sigma \downarrow\right)\right) \\
& =\delta_{\gamma}\left([u]_{i} \sigma \downarrow\right) .
\end{aligned}
$$

Case $f=h$ : This case is analogous to the previous one and can be handled in a similar way.

Case $\mathrm{f} \in\{\mathrm{pk}, \mathrm{vk},\langle\rangle\}$ : In such a case, we have that:

- $[u]_{i}=\mathrm{f}\left(\left[u_{1}\right]_{i}, \ldots,\left[u_{n}\right]_{i}\right)$ with $n \in\{1,2\}$, and

- $\operatorname{test}_{i}\left([u]_{i}\right)=\wedge_{j=1}^{n} \operatorname{test}_{i}\left(\left[u_{j}\right]_{i}\right)$.

We have that $[u]_{i} \sigma \downarrow=\mathrm{f}\left(\left[u_{1}\right]_{i} \sigma \downarrow, \ldots,\left[u_{n}\right]_{i} \sigma \downarrow\right)$. Thus, this case is similar to the senc case and can be handled similarly.

Case $\mathrm{f} \in\{$ sdec, adec, check $\}$ : In such a case, we have that:

- $[u]_{i}=\operatorname{untag}_{i}\left(\mathrm{f}\left(\left[u_{1}\right]_{i},\left[u_{2}\right]_{i}\right)\right)$, and

- test $_{i}\left([u]_{i}\right)$ is the following formula:

$$
\begin{aligned}
& \left(\operatorname{tag}_{i}\left(\operatorname{untag}_{i}\left(\mathrm{f}\left(\left[u_{1}\right]_{i},\left[u_{2}\right]_{i}\right)\right)\right)=\mathrm{f}\left(\left[u_{1}\right]_{i},\left[u_{2}\right]_{i}\right)\right) \\
& \wedge \operatorname{test}_{i}\left(\left[u_{1}\right]_{i}\right) \wedge \operatorname{test}_{i}\left(\left[u_{2}\right]_{i}\right)
\end{aligned}
$$

By hypothesis, we have that $\sigma \vDash \operatorname{test}_{i}\left([u]_{i}\right)$, thus we have that $\operatorname{tag}_{i}\left(\operatorname{untag}_{i}\left(\mathrm{f}\left(\left[u_{1}\right]_{i},\left[u_{2}\right]_{i}\right)\right)\right) \sigma \downarrow=\mathrm{f}\left(\left[u_{1}\right]_{i},\left[u_{2}\right]_{i}\right) \sigma \downarrow$. Hence, we deduce that the root function symbol $\mathrm{f}$ can be reduced and the root of the plaintext is $\operatorname{tag}_{i}$. More formally, there exist $v_{1}, v_{2}$ such that:

- $\mathrm{f}=\operatorname{sdec}:\left[u_{1}\right]_{i} \sigma \downarrow=\operatorname{senc}\left(\operatorname{tag}_{i}\left(v_{1}\right), v_{2}\right),\left[u_{2}\right]_{i} \sigma \downarrow=v_{2}$ and $[u]_{i} \sigma \downarrow=v_{1}$. This implies that:

$$
\delta_{\gamma}\left(\left[u_{1}\right]_{i} \sigma \downarrow\right)=\operatorname{senc}\left(\operatorname{tag}_{i}\left(\delta_{\gamma}\left(v_{1}\right)\right), \delta_{\gamma}\left(v_{2}\right)\right) .
$$

Thus, we can deduce that:

$$
\begin{aligned}
\operatorname{untag}_{i}\left(\operatorname{sdec}\left(\delta_{\gamma}\left(\left[u_{1}\right]_{i} \sigma \downarrow\right), \delta_{\gamma}\left(\left[u_{2}\right]_{i} \sigma \downarrow\right)\right)\right) \downarrow & =\delta_{\gamma}\left(v_{1}\right) \\
& =\delta_{\gamma}\left([u]_{i} \sigma \downarrow\right)
\end{aligned}
$$

- $\mathrm{f}=\operatorname{adec}:\left[u_{1}\right]_{i} \sigma \downarrow=\operatorname{aenc}\left(\operatorname{tag}_{i}\left(v_{1}\right), \operatorname{pk}\left(v_{2}\right)\right),\left[u_{2}\right]_{i} \sigma \downarrow=$ $v_{2}$, and $[u]_{i} \sigma \downarrow=v_{1}$.

- $\mathrm{f}=$ check: $\left[u_{1}\right]_{i} \sigma \downarrow=\operatorname{sign}\left(\operatorname{tag}_{i}\left(v_{1}\right), v_{2}\right),\left[u_{2}\right]_{i} \sigma \downarrow=$ $\operatorname{vk}\left(v_{2}\right)$, and $[u]_{i} \sigma \downarrow=v_{1}$.

In each case, we have that:

$$
\operatorname{untag}_{i}\left(\mathrm{f}\left(\delta_{\gamma}\left(\left[u_{1}\right]_{i} \sigma \downarrow\right), \delta_{\gamma}\left(\left[u_{2}\right]_{i} \sigma \downarrow\right)\right)\right) \downarrow=\delta_{\gamma}\left([u]_{i} \sigma \downarrow\right) .
$$

By inductive hypothesis, we have $\delta_{\gamma}\left(\left[u_{k}\right]_{i} \sigma \downarrow\right)=\delta_{\gamma}\left(\left[u_{k}\right]_{i} \sigma\right) \downarrow$ with $k \in\{1,2\}$. We also have that:

$$
\delta_{\gamma}\left([u]_{i} \sigma\right) \downarrow=\operatorname{untag}_{i}\left(\mathrm{f}\left(\delta_{\gamma}\left(\left[u_{1}\right]_{i} \sigma\right) \downarrow, \delta_{\gamma}\left(\left[u_{2}\right]_{i} \sigma\right) \downarrow\right)\right) \downarrow .
$$

This allows us to conclude that

$$
\begin{aligned}
\delta_{\gamma}\left([u]_{i} \sigma\right) \downarrow & =\operatorname{untag}_{i}\left(\mathrm{f}\left(\delta_{\gamma}\left(\left[u_{1}\right]_{i} \sigma \downarrow\right), \delta_{\gamma}\left(\left[u_{2}\right]_{i} \sigma \downarrow\right)\right)\right) \downarrow \\
& =\delta_{\gamma}\left([u]_{i} \sigma \downarrow\right) .
\end{aligned}
$$

Case $\mathrm{f}=\operatorname{proj}_{j}, j=1,2$ : In such a case, we have that $n=1$, and $[u]_{i}=\mathrm{f}\left(\left[u_{1}\right]_{i}\right)$. Since $\sigma \vDash \operatorname{test}_{i}\left([u]_{i}\right)$, we have that there exist $v_{1}, v_{2}$ such that $\left[u_{1}\right]_{i} \sigma \downarrow=\left\langle v_{1}, v_{2}\right\rangle$ and so $\delta_{\gamma}\left([u]_{i} \sigma \downarrow\right)=\delta_{\gamma}\left(v_{j}\right)$. But by inductive hypothesis, we have that $\delta_{\gamma}\left(\left[u_{1}\right]_{i} \sigma\right) \downarrow=\delta_{\gamma}\left(\left[u_{1}\right]_{i} \sigma \downarrow\right)=\left\langle\delta_{\gamma}\left(v_{1}\right), \delta_{\gamma}\left(v_{2}\right)\right\rangle$. Hence, $\delta_{\gamma}\left([u]_{i} \sigma\right) \downarrow=\mathrm{f}\left(\delta_{\gamma}\left(\left[u_{1}\right]_{i} \sigma\right)\right) \downarrow=\mathrm{f}\left(\delta_{\gamma}\left(\left[u_{1}\right]_{i} \sigma\right) \downarrow\right) \downarrow=\delta_{\gamma}\left(v_{j}\right) \downarrow$. We have shown that $\delta_{\gamma}\left(v_{j}\right)=\delta_{\gamma}\left([u]_{i} \sigma \downarrow\right)$, thus by Lemma 37, $\delta_{\gamma}\left(v_{j}\right)$ is in normal form and which allows us to conclude.

Corollary 39: Let $u, v \in \mathcal{T}\left(\Sigma_{i} \cup \Sigma_{0}, \mathcal{N} \cup \mathcal{X}\right)$ for some $i \in\{1, \ldots, p\}$. Let $\gamma \in\{\alpha, \beta\}$ such that $i \in \gamma$. Assume that $f v(u) \cup f v(v) \subseteq \operatorname{dom}\left(\sigma_{0} \downarrow\right)$, and $\sigma_{0} \vDash \operatorname{test}_{i}\left([u]_{i}\right) \wedge \operatorname{test}_{i}\left([v]_{i}\right)$. Moreover, assume that $u, v$ do not use names in $\mathcal{E}_{\alpha} \uplus \mathcal{E}_{\beta}$.

$$
[u]_{i} \sigma_{0} \downarrow=[v]_{i} \sigma_{0} \downarrow \Leftrightarrow \delta_{\gamma}\left([u]_{i}\right) \delta_{\gamma}\left(\sigma_{0} \downarrow\right) \downarrow=\delta_{\gamma}\left([v]_{i}\right) \delta_{\gamma}\left(\sigma_{0} \downarrow\right) \downarrow .
$$

Proof: Thanks to Lemma 35, we have that

$$
[u]_{i} \sigma_{0} \downarrow=[v]_{i} \sigma_{0} \downarrow \Leftrightarrow \delta_{\gamma}\left([u]_{i} \sigma_{0} \downarrow\right)=\delta_{\gamma}\left([v]_{i} \sigma_{0} \downarrow\right) .
$$

Thanks to Lemma 38, we have that:

- $\delta_{\gamma}\left([u]_{i} \sigma_{0} \downarrow\right)=\delta_{\gamma}\left([u]_{i}\left(\sigma_{0} \downarrow\right)\right) \downarrow=\delta_{\gamma}\left([u]_{i}\right) \delta_{\gamma}\left(\sigma_{0} \downarrow\right) \downarrow$, and

- $\delta_{\gamma}\left([v]_{i} \sigma_{0} \downarrow\right)=\delta_{\gamma}\left([v]_{i}\left(\sigma_{0} \downarrow\right)\right) \downarrow=\delta_{\gamma}\left([v]_{i}\right) \delta_{\gamma}\left(\sigma_{0} \downarrow\right) \downarrow$.

This allows us to conclude.

Lemma 40: Let $u \in \mathcal{T}\left(\Sigma_{i} \cup \Sigma_{0}, \mathcal{N} \cup \mathcal{X}\right)$ for some $i \in$ $\{1, \ldots, p\}$. Let $\gamma \in\{\alpha, \beta\}$ such that $i \in \gamma$. Assume that $f v(u) \subseteq \operatorname{dom}\left(\sigma_{0}\right)$. Moreover, assume that $u$ does not use names in $\mathcal{E}_{\alpha} \cup \mathcal{E}_{\beta}$. We have that :

$$
\sigma_{0} \downarrow \vDash \operatorname{test}_{i}\left([u]_{i}\right) \Leftrightarrow \delta_{\gamma}\left(\sigma_{0} \downarrow\right) \vDash \operatorname{test}_{i}\left(\delta_{\gamma}\left([u]_{i}\right)\right)
$$

Proof: To simplify the proof, we denote by $\sigma$ the substitution $\sigma_{0} \downarrow$. We prove this result by induction on $|u|$ :

Base case $|u|=1$ : In this case, we have that $u \in \mathcal{N} \cup \mathcal{X}$, and thus $[u]_{i}, \delta_{\gamma}\left([u]_{i}\right) \in \mathcal{N} \cup \mathcal{X}$. In such a case, we have that test $_{i}\left([u]_{i}\right)=$ true and test ${ }_{i}\left(\delta_{\gamma}\left([u]_{i}\right)\right)=$ true. Hence, the result trivially holds.

Inductive step $|u|>1$, i.e. $u=\mathrm{f}\left(u_{1}, \ldots, u_{n}\right)$. We do a case analysis on $\mathrm{f}$ :

Case $\mathrm{f} \in \Sigma_{i} \cup\{\mathrm{pk}, \mathrm{vk},\langle\rangle\}:$ In this case, we have that $[u]_{i}=$ $\mathrm{f}\left(\left[u_{1}\right]_{i}, \ldots,\left[u_{n}\right]_{i}\right)$ and $\delta_{\gamma}\left([u]_{i}\right)=\mathrm{f}\left(\delta_{\gamma}\left(\left[u_{1}\right]_{i}\right), \ldots, \delta_{\gamma}\left(\left[u_{n}\right]_{i}\right)\right)$. Thus, we deduce that $\operatorname{test}_{i}\left([u]_{i}\right)=\bigwedge_{j=1}^{n} \operatorname{test}_{i}\left(\left[u_{j}\right]_{i}\right)$ and test $_{i}\left(\delta_{\gamma}\left([u]_{i}\right)\right)=\bigwedge_{j=1}^{n} \operatorname{test}_{i}\left(\delta_{\gamma}\left(\left[u_{j}\right]_{i}\right)\right)$. By inductive hypothesis on $u_{1}, \ldots, u_{n}$, the result holds.

Case $\mathrm{f} \in\{$ senc, aenc, sign $\}:$ In this case, we have that:

- $[u]_{i}=\mathrm{f}\left(\operatorname{tag}_{i}\left(\left[u_{1}\right]_{i}\right),\left[u_{2}\right]_{i}\right)$, and

$$
\text { - } \delta_{\gamma}\left([u]_{i}\right)=\mathrm{f}\left(\operatorname{tag}_{i}\left(\delta_{\gamma}\left(\left[u_{1}\right]_{i}\right)\right), \delta_{\gamma}\left(\left[u_{2}\right]_{i}\right)\right) \text {. }
$$

Thus, we deduce that test ${ }_{i}\left([u]_{i}\right)=$ test $_{i}\left(\left[u_{1}\right]_{i}\right) \wedge$ test $_{i}\left(\left[u_{2}\right]_{i}\right)$ and $\operatorname{test}_{i}\left(\delta_{\gamma}\left([u]_{i}\right)\right)=\operatorname{test}_{i}\left(\delta_{\gamma}\left(\left[u_{1}\right]_{i}\right)\right) \wedge \operatorname{test}_{i}\left(\delta_{\gamma}\left(\left[u_{2}\right]_{i}\right)\right)$. By inductive hypothesis on $u_{1}, u_{2}$, the result holds.

Case $\mathrm{f}=\mathrm{h}$ : This case is analogous to de previous one and can be handled in a similar way.

Case $f \in\{\mathrm{sdec}$, adec, check $\}$ : In this case, we have that:

- $[u]_{i}=\operatorname{untag}_{i}\left(\mathrm{f}\left(\left[u_{1}\right]_{i},\left[u_{2}\right]_{i}\right)\right)$, and

- $\delta_{\gamma}\left([u]_{i}\right)=\operatorname{untag}_{i}\left(\mathrm{f}\left(\delta_{\gamma}\left(\left[u_{1}\right]_{i}\right), \delta_{\gamma}\left(\left[u_{2}\right]_{i}\right)\right)\right)$.

Thus, we deduce that $\operatorname{test}_{i}\left([u]_{i}\right)$ is the following formula:

$$
\begin{aligned}
& \operatorname{test}_{i}\left(\left[u_{1}\right]_{i}\right) \wedge \operatorname{test}_{i}\left(\left[u_{2}\right]_{i}\right) \\
& \wedge \operatorname{tag}_{i}\left(\operatorname{untag}_{i}\left(\mathrm{f}\left(\left[u_{1}\right]_{i},\left[u_{2}\right]_{i}\right)\right)\right)=\mathrm{f}\left(\left[u_{1}\right]_{i},\left[u_{2}\right]_{i}\right)
\end{aligned}
$$


and $\operatorname{test}_{i}\left(\delta_{\gamma}\left([u]_{i}\right)\right)$ is the following formula:

$\operatorname{test}_{i}\left(\delta_{\gamma}\left(\left[u_{1}\right]_{i}\right)\right) \wedge \operatorname{test}_{i}\left(\delta_{\gamma}\left(\left[u_{2}\right]_{i}\right)\right) \wedge$

$\operatorname{tag}_{i}\left(\operatorname{untag}_{i}\left(\mathrm{f}\left(\delta_{\gamma}\left(\left[u_{1}\right]_{i}\right), \delta_{\gamma}\left(\left[u_{2}\right]_{i}\right)\right)\right)\right)=\mathbf{f}\left(\delta_{\gamma}\left(\left[u_{1}\right]_{i}\right), \delta_{\gamma}\left(\left[u_{2}\right]_{i}\right)\right)$

Whether we assume that $\sigma \vDash \operatorname{test}_{i}\left([u]_{i}\right)$ or $\delta_{\gamma}(\sigma) \vDash$ $\operatorname{test}_{i}\left(\delta_{\gamma}\left([u]_{i}\right)\right)$, we have by inductive hypothesis that $\sigma \vDash$ test $_{i}\left(\left[u_{k}\right]_{i}\right)$ with $k \in\{1,2\}$. Thus by Lemma 38, it implies that $\delta_{\gamma}\left(\left[u_{k}\right]_{i} \sigma \downarrow\right)=\delta_{\gamma}\left(\left[u_{k}\right]_{i}\right) \delta_{\gamma}(\sigma) \downarrow$ with $k \in\{1,2\}$. We do a case analysis on $f$. We detail below the case where $f=$ sdec. The cases where $f=$ adec, and $f=$ check can be done in a similar way.

In such a case $(f=s d e c)$, we have that

$$
\sigma \vDash \operatorname{tag}_{i}\left(\operatorname{untag}_{i}\left(\mathrm{f}\left(\left[u_{1}\right]_{i},\left[u_{2}\right]_{i}\right)\right)\right)=\mathrm{f}\left(\left[u_{1}\right]_{i},\left[u_{2}\right]_{i}\right)
$$

is equivalent to there exists $v_{1}, v_{2}$ such that $\left[u_{2}\right]_{i} \sigma \downarrow=v_{2}$ and $\left[u_{1}\right]_{i} \sigma \downarrow=\operatorname{senc}\left(\operatorname{tag}_{i}\left(v_{1}\right), v_{2}\right)$. But by Lemma 35 , it is equivalent to $\delta_{\gamma}\left(\left[u_{1}\right]_{i} \sigma \downarrow\right)=\operatorname{senc}\left(\operatorname{tag}_{i}\left(\delta_{\gamma}\left(v_{1}\right)\right), \delta_{\gamma}\left(v_{2}\right)\right)$ and $\delta_{\gamma}\left(\left[u_{2}\right]_{i} \sigma \downarrow\right)=\delta_{\gamma}\left(v_{2}\right)$. Thus, it is equivalent to:

- $\delta_{\gamma}\left(\left[u_{1}\right]_{i}\right) \delta_{\gamma}(\sigma) \downarrow=\operatorname{senc}\left(\operatorname{tag}_{i}\left(\delta_{\gamma}\left(v_{1}\right)\right), \delta_{\gamma}\left(v_{2}\right)\right)$, and

- $\delta_{\gamma}\left(\left[u_{2}\right]_{i}\right) \delta_{\gamma}(\sigma) \downarrow=\delta_{\gamma}\left(v_{2}\right)$.

Hence it is equivalent to

$$
\delta_{\gamma}(\sigma) \vDash\left(\begin{array}{l}
\operatorname{tag}_{i}\left(\operatorname{untag}_{i}\left(\mathrm{f}\left(\delta_{\gamma}\left(\left[u_{1}\right]_{i}\right), \delta_{\gamma}\left(\left[u_{2}\right]_{i}\right)\right)\right)\right) \\
=\mathrm{f}\left(\delta_{\gamma}\left(\left[u_{1}\right]_{i}\right), \delta_{\gamma}\left(\left[u_{2}\right]_{i}\right)\right)
\end{array}\right)
$$

Case $f \in\left\{\operatorname{proj}_{1}\right.$, $\left.\operatorname{proj}_{2}\right\}:$ In such a case, we have that $[u]_{i}=$ $\mathrm{f}\left(\left[u_{1}\right]_{i}\right)$ and $\delta_{\gamma}\left([u]_{i}\right)=\mathrm{f}\left(\delta_{\gamma}\left(\left[u_{1}\right]_{i}\right)\right)$. Thus, we deduce that test $_{i}\left([u]_{i}\right)$ is the following formula:

$$
\operatorname{test}_{i}\left(\left[u_{1}\right]_{i}\right) \wedge\left\langle\operatorname{proj}_{1}\left(\left[u_{1}\right]_{i}\right), \operatorname{proj}_{2}\left(\left[u_{1}\right]_{i}\right)\right\rangle=\left[u_{1}\right]_{i}
$$

and $\operatorname{test}_{i}\left(\delta_{\gamma}\left([u]_{i}\right)\right)$ is the following formula:

$$
\begin{aligned}
& \operatorname{test}_{i}\left(\delta_{\gamma}\left(\left[u_{1}\right]_{i}\right)\right) \wedge \\
& \left\langle\operatorname{proj}_{1}\left(\delta_{\gamma}\left(\left[u_{1}\right]_{i}\right)\right), \operatorname{proj}_{2}\left(\delta_{\gamma}\left(\left[u_{1}\right]_{i}\right)\right)\right\rangle=\delta_{\gamma}\left(\left[u_{1}\right]_{i}\right)
\end{aligned}
$$

Whether we assume that $\sigma \vDash \operatorname{test}_{i}\left([u]_{i}\right)$ or $\delta_{\gamma}(\sigma) \vDash$ test $_{i}\left(\delta_{\gamma}\left([u]_{i}\right)\right)$, we have by inductive hypothesis that $\sigma \vDash$ test $_{i}\left(\left[u_{1}\right]_{i}\right)$. Thus by Lemma 38, it implies that $\delta_{\gamma}\left(\left[u_{1}\right]_{i} \sigma \downarrow\right)=$ $\delta_{\gamma}\left(\left[u_{1}\right]_{i}\right) \delta_{\gamma}(\sigma) \downarrow$.

Actually $\sigma \vDash\left\langle\operatorname{proj}_{1}\left(\left[u_{1}\right]_{i}\right), \operatorname{proj}_{2}\left(\left[u_{1}\right]_{i}\right)\right\rangle=\left[u_{1}\right]_{i}$ is equivalent to there exist $v_{1}, v_{2}$ such that $\left[u_{1}\right]_{i} \sigma \downarrow=\left\langle v_{1}, v_{2}\right\rangle$, which is, thanks to Lemma 35, equivalent to $\delta_{\gamma}\left(\left[u_{1}\right]_{i} \sigma \downarrow\right)=$ $\left\langle\delta_{\gamma}\left(v_{1}\right), \delta_{\gamma}\left(v_{2}\right)\right\rangle$.

We have shown that this is equivalent to

$$
\delta_{\gamma}\left(\left[u_{1}\right]_{i}\right) \delta_{\gamma}(\sigma) \downarrow=\left\langle\delta_{\gamma}\left(v_{1}\right), \delta_{\gamma}\left(v_{2}\right)\right\rangle
$$

Thus, we conclude that $\sigma \vDash\left\langle\operatorname{proj}_{1}\left(\left[u_{1}\right]_{i}\right)\right.$, $\left.\operatorname{proj}_{2}\left(\left[u_{1}\right]_{i}\right)\right\rangle=\left[u_{1}\right]_{i}$ is equivalent $\delta_{\gamma}(\sigma) \vDash\left\langle\operatorname{proj}_{1}\left(\delta_{\gamma}\left(\left[u_{1}\right]_{i}\right)\right)\right.$, $\left.\operatorname{proj}_{2}\left(\delta_{\gamma}\left(\left[u_{1}\right]_{i}\right)\right)\right\rangle=$ $\delta_{\gamma}\left(\left[u_{1}\right]_{i}\right)$.

For a term $u$ that does not contain any tag, we defined a way to construct a term that is properly tagged (i.e. $[u]_{i}$ ). Hence, for a term properly tagged, we would never have $\operatorname{senc}(n, k)$ where $n$ and $k$ are both nonces, for example. Instead, we would have $\operatorname{senc}\left(\operatorname{tag}_{i}(n), k\right)$. However, even if we can force the processes to properly tag their terms, we do not have any control on what the intruder can build. Typically, if the intruder is able to deduce $n$ and $k$, he is allowed to send to a process the term senc $(n, k)$. Thus, we want to define the notion of flawed tagged term.

Definition 41: Let $u$ be a ground term in normal form. Consider $\gamma$ and $\gamma^{\prime}$ such that $\left\{\gamma, \gamma^{\prime}\right\}=\{\alpha, \beta\}$. We define the flawed subterms of $u$ w.r.t. $\gamma$, denoted Flawed ${ }^{\gamma}(u)$, as follows:

$$
\text { Flawed }^{\gamma}(u) \stackrel{\text { def }}{=}\left\{\begin{array}{l|l}
v \in \operatorname{st}(u) \mid \begin{array}{l}
\operatorname{tagroot}(v) \in\{0\} \cup \gamma^{\prime} \text { and } \\
\operatorname{root}(v) \notin\{\mathrm{pk}, \mathrm{vk},\langle\rangle\}
\end{array}
\end{array}\right\}
$$

We define the flawed subterms of $u$, denoted Flawed $(u)$, as the set $\operatorname{Flawed}(u)=$ Flawed $^{\alpha}(u) \cap$ Flawed $^{\beta}(u)$

Lemma 42: Let $u \in \mathcal{T}\left(\Sigma_{i} \cup \Sigma_{0}, \mathcal{N} \cup \mathcal{X}\right)$ for some $i \in$ $\{1, \ldots, p\}$. Let $\gamma \in\{\alpha, \beta\}$ such that $i \in \gamma$. Let $\gamma^{\prime}$ such that $\gamma^{\prime} \in\{\alpha, \beta\} \backslash \gamma$. Let $\sigma$ be a ground substitution in normal form such that $f v(u) \subseteq \operatorname{dom}(\sigma)$.

If $\sigma \vDash \operatorname{test}_{i}\left([u]_{i}\right)$ then for all $t \in \operatorname{Flawed}^{\gamma}\left([u]_{i} \sigma \downarrow\right)$, there exists $x \in f v\left([u]_{i}\right)$ such that $t \in \operatorname{Flawed}^{\gamma}(x \sigma)$.

Proof: We prove the result by induction on $|u|$.

Base case $|u|=1$ : In this case, we have that $u \in \mathcal{X} \cup \mathcal{N}$ and so $[u]_{i}=u$. If $u \in \mathcal{N}$, then $u \sigma$ and $[u]_{i} \sigma \downarrow$ are both in $\mathcal{N}$, which means that Flawed $^{\gamma}\left([u]_{i} \sigma \downarrow\right)=\emptyset$. Thus, the result holds. Otherwise, we have that $u \in \mathcal{X}$ and so $[u]_{i}=u \in \operatorname{dom}(\sigma)$ which means that the result trivially holds.

Inductive step $|u|>1$, i.e. $u=\mathrm{f}\left(u_{1}, \ldots, u_{n}\right)$. We do a case analysis on $f$.

Case $f \in \Sigma_{i}$ : In this case, $[u]_{i}=\mathrm{f}\left(\left[u_{1}\right]_{i}, \ldots,\left[u_{n}\right]_{i}\right)$ and $[u]_{i} \sigma \downarrow=\mathrm{f}\left(\left[u_{1}\right]_{i} \sigma \downarrow, \ldots,\left[u_{n}\right]_{i} \sigma \downarrow\right) \downarrow$. By definition, we know that for all $t \in$ Flawed $^{\gamma}\left([u]_{i} \sigma \downarrow\right), \operatorname{root}(t) \notin \Sigma_{\gamma}$. Thus, thanks to Lemma 26, for all $t \in \operatorname{Flawed}^{\gamma}\left([u]_{i} \sigma \downarrow\right)$, there exists $k \in\{1, \ldots, n\}$ such that $t \in s t\left(\left[u_{k}\right]_{i} \sigma \downarrow\right)$. By hypothesis, $\sigma \vDash \operatorname{test}_{i}\left([u]_{i}\right)$ and so $\sigma \vDash \operatorname{test}_{i}\left(\left[u_{k}\right]_{i}\right)$. Thus, by inductive hypothesis, we know that there exists $x \in f v\left(\left[u_{k}\right]_{i}\right)$ such that $t \in \operatorname{st}(x \sigma)$. Since $f v\left(\left[u_{k}\right]_{i}\right) \subseteq f v\left([u]_{i}\right)$, we can conclude.

Case $f \in$ \{senc, aenc, sign $\}$ : In such a case, $[u]_{i}=$ $\mathrm{f}\left(\operatorname{tag}_{i}\left(\left[u_{1}\right]_{i}\right),\left[u_{2}\right]_{i}\right)$ and $[u]_{i} \sigma \downarrow=\mathrm{f}\left(\operatorname{tag}_{i}\left(\left[u_{1}\right]_{i} \sigma \downarrow\right),\left[u_{2}\right]_{i} \sigma \downarrow\right)$. Moreover, $\sigma \vDash \operatorname{test}_{i}\left([u]_{i}\right)$ implies that $\sigma \vDash \operatorname{test}_{i}\left(\left[u_{k}\right]_{i}\right)$, with $k \in\{1,2\}$. Since tagroot $\left([u]_{i} \sigma \downarrow\right)=i$, then we deduce that :

$$
\text { Flawed }^{\gamma}\left([u]_{i} \sigma \downarrow\right)=\text { Flawed }^{\gamma}\left(\left[u_{1}\right]_{i} \sigma \downarrow\right) \cup \text { Flawed }^{\gamma}\left(\left[u_{2}\right]_{i} \sigma \downarrow\right)
$$

Thanks to our inductive hypothesis on $u_{1}$ and $u_{2}$, the result holds.

Case $\mathrm{f}=\mathrm{h}$ : This case is analogous to the previous one and can be handled in a similar way.

Case $\mathrm{f}=\langle\rangle:$ In this case, we have that $[u]_{i}=\mathrm{f}\left(\left[u_{1}\right]_{i},\left[u_{2}\right]_{i}\right)$, and $[u]_{i} \sigma \downarrow=\mathrm{f}\left(\left[u_{1}\right]_{i} \sigma \downarrow,\left[u_{2}\right]_{i} \sigma \downarrow\right)$. Moreover, $\sigma \vDash \operatorname{test}_{i}\left([u]_{i}\right)$ implies that $\sigma \vDash \operatorname{test}_{i}\left(\left[u_{k}\right]_{i}\right)$ with $k \in\{1,2\}$. By definition, since $\operatorname{root}\left([u]_{i} \sigma \downarrow\right)=\langle\rangle$, we have that Flawed $^{\gamma}\left([u]_{i} \sigma \downarrow\right)=$ Flawed $^{\gamma}\left(\left[u_{1}\right]_{i} \sigma \downarrow\right) \cup$ Flawed $^{\gamma}\left(\left[u_{2}\right]_{i} \sigma \downarrow\right)$. Applying our inductive hypothesis on $u_{1}$ and $u_{2}$, we conclude.

Case $\mathrm{f}=\{\mathrm{vk}, \mathrm{pk}\}$ : In this case, we have $u=\mathrm{f}(v)$ with $v \in$ $\mathcal{N} \cup \mathcal{X}$. Thus $[u]_{i}=u$ and so by definition, Flawed $^{\gamma}(u \sigma \downarrow)=\emptyset$. Thus, the result trivially holds. 
Case $f \in\{$ sdec, adec, check $\}$ : In this case, we have that $[u]_{i}=\operatorname{untag}_{i}\left(\mathrm{f}\left(\left[u_{1}\right]_{i},\left[u_{2}\right]_{i}\right)\right)$ and

$$
\begin{aligned}
\operatorname{test}_{i}\left([u]_{i}\right)= & \operatorname{test}_{i}\left(\left[u_{1}\right]_{i}\right) \wedge \operatorname{test}_{i}\left(\left[u_{2}\right]_{i}\right) \wedge \\
& \operatorname{tag}_{i}\left([u]_{i}\right)=\mathrm{f}\left(\left[u_{1}\right]_{i},\left[u_{2}\right]_{i}\right) .
\end{aligned}
$$

By hypothesis, we know that $\sigma \vDash \operatorname{test}_{i}\left([u]_{i}\right)$ and more specifically $\operatorname{tag}_{i}\left([u]_{i}\right) \sigma \downarrow=\mathrm{f}\left(\left[u_{1}\right]_{i},\left[u_{2}\right]_{i}\right) \sigma \downarrow$. It implies that there exist $v_{1}, v_{2}$ such that $\left[u_{1}\right]_{i} \sigma \downarrow=\mathrm{g}\left(\operatorname{tag}_{i}\left(v_{1}\right), v_{2}\right)$ and $[u]_{i} \sigma \downarrow=v_{1}$, with $\mathrm{g} \in$ \{senc, aenc, sign $\}$. Thus, for all $t \in \operatorname{Flawed}^{\gamma}\left([u]_{i} \sigma \downarrow\right), t \in \operatorname{Flawed}^{\gamma}\left(\left[u_{1}\right]_{i} \sigma \downarrow\right)$. Since $\sigma \vDash$ test $_{i}\left(\left[u_{1}\right]_{i}\right)$, the result holds by inductive hypothesis.

Case $\mathrm{f}=\operatorname{proj}_{j}, j \in\{1,2\}$ : We have that $[u]_{i}=\mathrm{f}\left(\left[u_{1}\right]_{i}\right)$ and $\operatorname{test}_{i}\left([u]_{i}\right)=\operatorname{test}_{i}\left(\left[u_{1}\right]_{i}\right) \wedge\left\langle\operatorname{proj}_{1}\left(\left[u_{1}\right]_{i}\right), \operatorname{proj}_{2}\left(\left[u_{1}\right]_{i}\right)\right\rangle=\left[u_{1}\right]_{i}$. Hence, $\sigma \vDash \operatorname{test}_{i}\left([u]_{i}\right)$ implies that there exist $v_{1}, v_{2}$ such that $\left[u_{1}\right]_{i} \sigma \downarrow=\left\langle v_{1}, v_{2}\right\rangle$ and $[u]_{i} \sigma \downarrow=v_{j}$. Thus, for all $t \in \operatorname{Flawed}^{\gamma}\left([u]_{i} \sigma \downarrow\right), t \in \operatorname{Flawed}^{\gamma}\left(\left[u_{1}\right]_{i} \sigma \downarrow\right)$. Since $\sigma \vDash$ test $_{i}\left(\left[u_{1}\right]_{i}\right)$, our inductive hypothesis allows us to conclude.

Corollary 43: Let $u \in \mathcal{T}\left(\Sigma_{i} \cup \Sigma_{0}, \mathcal{N} \cup \mathcal{X}\right)$ for some $i \in$ $\{1, \ldots, p\}$. Let $\gamma \in\{\alpha, \beta\}$ such that $i \in \gamma$. Let $\sigma$ be a ground substitution in normal form such that $f v(u) \subseteq \operatorname{dom}(\sigma)$.

If $\sigma \vDash \operatorname{test}_{i}\left([u]_{i}\right)$ then for all $t \in \operatorname{Flawed}\left([u]_{i} \sigma \downarrow\right)$, there exists $x \in f v\left([u]_{i}\right)$ such that $t \in \operatorname{Flawed}(x \sigma)$.

Corollary 44: Let $u \in \mathcal{T}\left(\Sigma_{i} \cup \Sigma_{0}, \mathcal{N} \cup \mathcal{X}\right)$ for some $i \in\{1, \ldots, p\}$. Let $\gamma \in\{\alpha, \beta\}$ such that $i \in \gamma$. Assume that $f v(u) \subseteq \operatorname{dom}\left(\sigma_{0}\right)$. Moreover, assume that $u$ does not use names in $\mathcal{E}_{\alpha} \cup \mathcal{E}_{\beta}$. If $\delta_{\gamma}\left(\sigma_{0} \downarrow\right) \vDash$ test $_{i}\left(\delta_{\gamma}\left([u]_{i}\right)\right)$, then for all $t \in$ Flawed $^{\gamma}\left(\delta_{\gamma}\left([u]_{i}\right) \delta_{\gamma}\left(\sigma_{0} \downarrow\right) \downarrow\right)$, there exists $x \in f v\left(\delta_{\gamma}\left([u]_{i}\right)\right)$ such that $t \in$ Flawed $^{\gamma}\left(x \delta_{\gamma}\left(\sigma_{0} \downarrow\right)\right)$.

Proof: By Lemma 37, we deduce that $\delta_{\gamma}\left(\sigma_{0} \downarrow\right)$ is a substitution in normal form. Moreover, since $u \in \mathcal{T}\left(\Sigma_{i} \cup \Sigma_{0}, \mathcal{N} \cup \mathcal{X}\right)$ and by definition of $\delta_{\gamma}$, and [ $]_{i}$, we deduce that there exists $v \in$ $\mathcal{T}\left(\Sigma_{i} \cup \Sigma_{0}, \mathcal{N} \cup \mathcal{X}\right)$ such that $[v]_{i}=\delta_{\gamma}\left([u]_{i}\right)$. By application of Lemma 42, we deduce that for all $t \in \operatorname{Flawed}^{\gamma}\left([v]_{i} \delta_{\gamma}\left(\sigma_{0} \downarrow\right) \downarrow\right)$, there exists $x \in f v\left([v]_{i}\right)$ such that $t \in s t\left(x \delta_{\gamma}\left(\sigma_{0} \downarrow\right)\right)$. Hence, we conclude that for all $t \in \operatorname{Flawed}^{\gamma}\left(\delta_{\gamma}\left([u]_{i}\right) \delta_{\gamma}\left(\sigma_{0} \downarrow\right) \downarrow\right)$, there exists $x \in f v\left(\delta_{\gamma}\left([u]_{i}\right)\right)$ such that $t \in \operatorname{st}\left(x \delta_{\gamma}\left(\sigma_{0} \downarrow\right)\right)$.

Definition 45: Let $u \in \mathcal{T}(\Sigma, \mathcal{N} \cup \mathcal{X})$. The $\alpha$-factors (resp. $\beta$-factors) of $u$, denoted $F c t_{\alpha}(u)$, are the maximal syntactic subterms of $u$ that are also in Flawed $^{\alpha}(u)\left(\right.$ resp. Flawed $\left.{ }^{\beta}(u)\right)$.

Lemma 46: Let $u \in \mathcal{T}\left(\Sigma_{i} \cup \Sigma_{0}, \mathcal{N} \cup \mathcal{X}\right)$ for some $i \in$ $\{1, \ldots, p\}$. Let $\gamma \in\{\alpha, \beta\}$ such that $i \in \gamma$. Let $\sigma$ be a ground substitution in normal form such that $f v(u) \subseteq \operatorname{dom}(\sigma)$.

If $\sigma \vDash \operatorname{test}_{i}\left([u]_{i}\right)$ then

- either $[u]_{i} \sigma \downarrow \in F c t_{\gamma}\left([u]_{i} \sigma\right)$,

- otherwise $F_{c t}\left([u]_{i} \sigma \downarrow\right) \subseteq F_{c t}\left([u]_{i} \sigma\right)$

Proof: We prove the result by induction on $|u|$.

Base case $|u|=1$ : In this case, we have that $u \in \mathcal{X} \cup \mathcal{N}$ and so $[u]_{i}=u$. If $u \in \mathcal{N}$, then $u \sigma$ and $[u]_{i} \sigma \downarrow$ are both in $\mathcal{N}$, which means that $\operatorname{Fct}_{\gamma}\left([u]_{i} \sigma\right)=\emptyset$ and $F_{c t}\left([u]_{i} \sigma \downarrow\right)=\emptyset$. Thus, the result holds. Otherwise, we have that $u \in \mathcal{X}$ and so $[u]_{i}=u$. But $\sigma$ is in normal form hence $[u]_{i} \sigma \downarrow=[u]_{i} \sigma$. Thus, $F c t_{\alpha}\left([u]_{i} \sigma \downarrow\right)=F c t_{\alpha}\left([u]_{i} \sigma \downarrow\right)$ and so the result holds.
Inductive step $|u|>1$, i.e. $u=\mathrm{f}\left(u_{1}, \ldots, u_{n}\right)$. We do a case analysis on $\mathrm{f}$.

Case $\mathrm{f} \in \Sigma_{i}$ : In this case, $[u]_{i}=\mathrm{f}\left(\left[u_{1}\right]_{i}, \ldots,\left[u_{n}\right]_{i}\right)$ and $[u]_{i} \sigma \downarrow=\mathrm{f}\left(\left[u_{1}\right]_{i} \sigma \downarrow, \ldots,\left[u_{n}\right]_{i} \sigma \downarrow\right) \downarrow$.

By definition, we know that for all $t \in \operatorname{Fct}_{\gamma}[u]_{i} \sigma \downarrow$, $\operatorname{root}(t) \notin \Sigma_{\gamma}$. Thus, thanks to Lemma 26, for all $t \in$ $F c t_{\gamma}[u]_{i} \sigma \downarrow$, there exists $k \in\{1, \ldots, n\}$ such that $t \in$ $\operatorname{Fct}_{\gamma}\left(\left[u_{k}\right]_{i} \sigma \downarrow\right)$. By hypothesis, $\sigma \vDash$ test $_{i}\left([u]_{i}\right)$ and so $\sigma \vDash$ test $_{i}\left(\left[u_{k}\right]_{i}\right)$. Thus, by inductive hypothesis, we know that

- either $\left[u_{k}\right]_{i} \sigma \downarrow \in F c t_{\gamma}\left(\left[u_{k}\right]_{i} \sigma\right)$,

- otherwise $F_{c t}\left(\left[u_{k}\right]_{i} \sigma \downarrow\right) \subseteq F_{c t}\left(\left[u_{k}\right]_{i} \sigma\right)$

Thus, if $\left[u_{k}\right]_{i} \sigma \downarrow \in \operatorname{Fct}_{\gamma}\left(\left[u_{k}\right]_{i} \sigma\right)$ then it means that $t=$ $\left[u_{k}\right]_{i} \sigma \downarrow$ and so $t \in F_{c t}\left(\left[u_{k}\right]_{i} \sigma\right)$ (otherwise it contradicts the notion of maximal subterm). Thus in both cases, we obtain that $t \in F c t_{\gamma}\left(\left[u_{k}\right]_{i} \sigma\right)$. Since $F c t_{\gamma}\left(\left[u_{k}\right]_{i} \sigma\right) \subseteq F c t_{\gamma}\left([u]_{i} \gamma\right)$ then we deduce that $t \in F_{c t}\left([u]_{i} \gamma\right)$ hence the result holds.

Case $f \in$ \{senc, aenc, sign $\}$ : In such a case, $[u]_{i}=$ $\mathrm{f}\left(\operatorname{tag}_{i}\left(\left[u_{1}\right]_{i}\right),\left[u_{2}\right]_{i}\right)$ and $[u]_{i} \sigma \downarrow=\mathrm{f}\left(\operatorname{tag}_{i}\left(\left[u_{1}\right]_{i} \sigma \downarrow\right),\left[u_{2}\right]_{i} \sigma \downarrow\right)$. Moreover, $\sigma \vDash \operatorname{test}_{i}\left([u]_{i}\right)$ implies that $\sigma \vDash \operatorname{test}_{i}\left(\left[u_{k}\right]_{i}\right)$, with $k \in\{1,2\}$. Since $\operatorname{tagroot}\left([u]_{i} \sigma \downarrow\right)=i$, then we deduce that :

$$
F_{c t}\left([u]_{i} \sigma \downarrow\right)=F_{c t}\left(\left[u_{1}\right]_{i} \sigma \downarrow\right) \cup F c t_{\gamma}\left(\left[u_{2}\right]_{i} \sigma \downarrow\right)
$$

Thanks to our inductive hypothesis on $u_{1}$ and $u_{2}$, the result holds.

Case $\mathrm{f}=\mathrm{h}$ : This case is analogous to the previous one and can be handled in a similar way.

Case $\mathrm{f}=\langle\rangle$ : In this case, we have that $[u]_{i}=\mathrm{f}\left(\left[u_{1}\right]_{i},\left[u_{2}\right]_{i}\right)$, and $[u]_{i} \sigma \downarrow=\mathrm{f}\left(\left[u_{1}\right]_{i} \sigma \downarrow,\left[u_{2}\right]_{i} \sigma \downarrow\right)$. Moreover, $\sigma \vDash \operatorname{test}_{i}\left([u]_{i}\right)$ implies that $\sigma \vDash \operatorname{test}_{i}\left(\left[u_{k}\right]_{i}\right)$ with $k \in\{1,2\}$. By definition, since $\operatorname{root}\left([u]_{i} \sigma \downarrow\right)=\langle\rangle$, we have that $F_{c} t_{\gamma}\left([u]_{i} \sigma \downarrow\right)=$ $F c t_{\gamma}\left(\left[u_{1}\right]_{i} \sigma \downarrow\right) \cup F c t_{\gamma}\left(\left[u_{2}\right]_{i} \sigma \downarrow\right)$. Applying our inductive hypothesis on $u_{1}$ and $u_{2}$, we conclude.

Case $\mathrm{f}=\{\mathrm{vk}, \mathrm{pk}\}$ : In this case, we have $u=\mathrm{f}(v)$ with $v \in$ $\mathcal{N} \cup \mathcal{X}$. Thus $[u]_{i}=u$ and so by definition, $\operatorname{Fct}_{\gamma}(u \sigma \downarrow)=\emptyset$. Thus, the result trivially holds.

Case $f \in\{$ sdec, adec, check $\}$ : In this case, we have that $[u]_{i}=\operatorname{untag}_{i}\left(\mathrm{f}\left(\left[u_{1}\right]_{i},\left[u_{2}\right]_{i}\right)\right)$ and

$$
\begin{aligned}
\operatorname{test}_{i}\left([u]_{i}\right)= & \operatorname{test}_{i}\left(\left[u_{1}\right]_{i}\right) \wedge \operatorname{test}_{i}\left(\left[u_{2}\right]_{i}\right) \wedge \\
& \operatorname{tag}_{i}\left([u]_{i}\right)=\mathrm{f}\left(\left[u_{1}\right]_{i},\left[u_{2}\right]_{i}\right) .
\end{aligned}
$$

By hypothesis, we know that $\sigma \vDash$ test $_{i}\left([u]_{i}\right)$ and more specifically $\operatorname{tag}_{i}\left([u]_{i}\right) \sigma \downarrow=\mathrm{f}\left(\left[u_{1}\right]_{i},\left[u_{2}\right]_{i}\right) \sigma \downarrow$. It implies that there exists $v_{1}, v_{2}$ such that $\left[u_{1}\right]_{i} \sigma \downarrow=\mathrm{g}\left(\operatorname{tag}_{i}\left(v_{1}\right), v_{2}\right)$ and $[u]_{i} \sigma \downarrow=v_{1}$, with $\mathrm{g} \in$ \{senc, aenc, sign $\}$. Thus, for all $t \in F_{c t}\left([u]_{i} \sigma \downarrow\right), t \in F c t_{\gamma}\left(\left[u_{1}\right]_{i} \sigma \downarrow\right)$. Since $\sigma \vDash$ test $_{i}\left(\left[u_{1}\right]_{i}\right)$, the result holds by inductive hypothesis.

Case $\mathrm{f}=\operatorname{proj}_{j}, j \in\{1,2\}$ : We have that $[u]_{i}=\mathrm{f}\left(\left[u_{1}\right]_{i}\right)$ and $\operatorname{test}_{i}\left([u]_{i}\right)=\operatorname{test}_{i}\left(\left[u_{1}\right]_{i}\right) \wedge\left\langle\operatorname{proj}_{1}\left(\left[u_{1}\right]_{i}\right), \operatorname{proj}_{2}\left(\left[u_{1}\right]_{i}\right)\right\rangle=$ $\left[u_{1}\right]_{i}$. Hence, $\sigma \vDash \operatorname{test}_{i}\left([u]_{i}\right)$ implies that there exist $v_{1}, v_{2}$ such that $\left[u_{1}\right]_{i} \sigma \downarrow=\left\langle v_{1}, v_{2}\right\rangle$ and $[u]_{i} \sigma \downarrow=v_{j}$. Thus, for all $t \in F_{c t}\left([u]_{i} \sigma \downarrow\right), t \in F c t_{\gamma}\left(\left[u_{1}\right]_{i} \sigma \downarrow\right)$. Since $\sigma \vDash \operatorname{test}_{i}\left(\left[u_{1}\right]_{i}\right)$, our inductive hypothesis allows us to conclude. 


\section{E. Frame of a tagged process}

In this subsection, we will state and prove the lemmas regarding frames and static equivalence. Let $\nu \mathcal{E} . \Phi$ be a frame such that:

$$
\Phi=\left\{w_{1} \triangleright u_{1}, \ldots, w_{n} \triangleright u_{n}\right\}
$$

Let $M$ be a recipe, i.e. a term such that $f v(M) \subseteq \operatorname{dom}(\Phi)$ and $f n(M) \cap \mathcal{E}=\emptyset$, we define the measure $\mu$ as follows:

$$
\mu(M)=\left(i_{\max },|M|\right)
$$

where $i_{\max } \in\{1, \ldots, n\}$ is the maximal indice $i$ such that $w_{i} \in f v(M)$, and $|M|$ denotes the size of the term $M$, i.e. the number of symbols that occur in $M$.

We have that $\mu\left(M_{1}\right) \stackrel{\text { def }}{=}\left(i_{1}, s_{1}\right)<\mu\left(M_{2}\right) \stackrel{\text { def }}{=}\left(i_{2}, s_{2}\right)$ when either $i_{1}<i_{2}$; or $i_{1}=i_{2}$ and $s_{1}<s_{2}$.

Once again, we denote by $z_{1}^{\alpha}, \ldots, z_{k}^{\alpha}$ and $z_{1}^{\beta}, \ldots, z_{\ell}^{\beta}$ the assignment variables of the extended processes that we are considering.

Definition 47: Let $(\mathcal{E} ; \mathcal{P} ; \Phi ; \sigma)$ be an extended process, $\prec$ be a total order on $\operatorname{dom}(\Phi) \cup \operatorname{dom}(\sigma)$ and col be a mapping from $\operatorname{dom}(\Phi) \cup \operatorname{dom}(\sigma)$ to $\{1, \ldots, p\}$. We say that $(\mathcal{E} ; \mathcal{P} ; \Phi ; \sigma)$ is a derived well-tagged extended process w.r.t. $\prec$ and col if for every $x \in \operatorname{dom}(\Phi)$ (resp. $x \in \operatorname{dom}(\sigma)$ ), there exists $\left\{\gamma, \gamma^{\prime}\right\}=\{\alpha, \beta\}$ such that one of the following condition is satisfied:

1) there exist $v$ and $i=\operatorname{col}(x) \in \gamma$ such that $u=[v]_{i} \sigma$, $\sigma \vDash \operatorname{test}_{i}\left([v]_{i}\right)$, and for all $z \in f v(v), z \prec x$ and either $\operatorname{col}(z) \in \gamma$ or there exists $j$ such that $z=z_{j}^{\gamma^{\prime}}$; or

2) there exists $M$ such that $f v(M) \subseteq \operatorname{dom}(\Phi) \cap\{z \mid z \prec$ $x\}, f n(M) \cap \mathcal{E}=\emptyset$ and $M \Phi=u$.

where $u=x \Phi$ (resp. $u=x \sigma$ ).

In the case of variables instantiated through an output, and or an internal communication, it will be the first item that needs to hold; while in the case of variables intantiated through inputs on public channels it is the second item that needs to hold. Intuitively, the order $\prec$ on $\operatorname{dom}(\Phi) \cup$ $\operatorname{dom}(\sigma)$ corresponds to the order in which the variables in $\operatorname{dom}(\Phi) \cup \operatorname{dom}(\sigma)$ have been introduced along the execution. In particular, we have that $w_{1} \prec w_{2} \prec \ldots \prec w_{n}$ where $\operatorname{dom}(\Phi)=\left\{w_{1}, \ldots, w_{n}\right\}$. In the following, we sometimes simply say that $(\mathcal{E} ; \mathcal{P} ; \Phi ; \sigma)$ is a derived well-tagged extended process.

Lemma 48: Let $(\mathcal{E} ; \mathcal{P} ; \Phi ; \sigma)$ be a derived well-tagged extended process w.r.t $\prec$ and col. Let $x \in \operatorname{dom}(\Phi)$ (resp. $x \in \operatorname{dom}(\sigma))$ and $t \in \operatorname{Flawed}(x \Phi \downarrow)$ (resp. $t \in \operatorname{Flawed}(x \sigma \downarrow))$. We have that there exists $M$ such that $f v(M) \subseteq \operatorname{dom}(\Phi) \cap$ $\{z \mid z \prec x\}, f n(M) \cap \mathcal{E}=\emptyset$ and $t \in \operatorname{Flawed}(M \Phi \downarrow)$.

Proof: We prove this result by induction on $\operatorname{dom}(\Phi) \cup$ $\operatorname{dom}(\sigma)$ with the order $\prec$.

Base case $u=x \sigma$ or $u=x \Phi$ with $x \prec z$ for any $z \in$ $\operatorname{dom}(\Phi) \cup \operatorname{dom}(\sigma)$. Assume $t \in \operatorname{Flawed}(u \downarrow)$. By definition of a derived well-tagged extended process w.r.t $\prec$ and $\mathrm{col}$, one of the following condition is satisfied:
1) There exist $v$ and $i=\operatorname{col}(x)$ such that $u=[v]_{i} \sigma, \sigma \vDash$ test $_{i}\left([v]_{i}\right)$, and $z \prec x$ for any $z \in f v(v)$. Since $u=$ $[v]_{i} \sigma$ and $\sigma \vDash$ test $_{i}\left([v]_{i}\right)$, we can apply Lemma 42 to $v$ and $\sigma \downarrow$. Thus, we have that there exists $z \in f v\left([v]_{i}\right)$ such that $t \in$ Flawed $(z \sigma \downarrow)$. However, since $x$ is mimimal w.r.t. $\prec$, we know that $f v(v)=\emptyset$. Hence, we obtain a contradiction. This case is impossible.

2) There exists $M$ such that $f v(M) \subseteq \operatorname{dom}(\Phi) \cap\{z \mid z \prec$ $x\}, f n(M) \cap \mathcal{E}=\emptyset$, and $M \Phi=u$. Thus, we have that $M \Phi \downarrow=u \downarrow$, and we have that $t \in \operatorname{Flawed}(M \Phi \downarrow)$.

Inductive case $u=x \sigma$ or $u=x \Phi$. Assume $t \in \operatorname{Flawed}(u \downarrow)$. By definition of a derived well-tagged extended process w.r.t $\prec$ and $\mathrm{col}$, one of the following condition is satisfied:

1) There exist $v$ and $i=\operatorname{col}(x)$ such that $u=[v]_{i} \sigma, \sigma \vDash$ test $_{i}\left([v]_{i}\right)$, and $z \prec x$ for any $z \in f v(v)$. Since $u=$ $[v]_{i} \sigma$ and $\sigma \vDash \operatorname{test}_{i}\left([v]_{i}\right)$, we can apply Lemma 42 to $v$ and $\sigma \downarrow$. Thus, we have that there exists $z \in f v\left([v]_{i}\right)$ such that $t \in \operatorname{Flawed}(z \sigma \downarrow)$, and we have that $z \prec x$. Hence, we conclude by applying our induction hypothesis.

2) There exists $M$ such that $f v(M) \subseteq \operatorname{dom}(\Phi) \cap\{z \mid z \prec$ $x\}, f n(M) \cap \mathcal{E}=\emptyset$, and $M \Phi=u$. Thus, we have that $M \Phi \downarrow=u \downarrow$, and we have that $t \in \operatorname{Flawed}(M \Phi \downarrow)$.

This allows us to conclude.

Lemma 49: Let $(\mathcal{E} ; \mathcal{P} ; \Phi ; \sigma)$ be a derived well-tagged extended process w.r.t $\prec$ and col. Let $\left\{\gamma, \gamma^{\prime}\right\}=\{\alpha, \beta\}$. Let $x \in \operatorname{dom}(\Phi)$ (resp. $x \in \operatorname{dom}(\sigma)$ ) such that $\operatorname{col}(x) \in \gamma$. Let $u=x \Phi$ (resp. $u=x \sigma$ ). Let $t \in F_{c t}(u \downarrow)$. We have that

- either there exists $M$ such that $f v(M) \subseteq \operatorname{dom}(\Phi) \cap$ $\{z \mid z \prec x\}, f n(M) \cap \mathcal{E}=\emptyset$ and $t \in \operatorname{Fct}_{\gamma}(M \Phi \downarrow)$;

- otherwise there exists $j$ such that $z_{j}^{\gamma^{\prime}} \prec x$ and $z_{j}^{\gamma^{\prime}} \sigma \downarrow=t$. Proof: We prove this result by induction on $\operatorname{dom}(\Phi) \cup$ $\operatorname{dom}(\sigma)$ with the order $\prec$.

Base case $u=x \sigma$ or $u=x \Phi$ with $x \prec z$ for any $z \in$ $\operatorname{dom}(\Phi) \cup \operatorname{dom}(\sigma)$. Let $t \in F c t_{\gamma}(u \downarrow)$ and $\operatorname{col}(x) \in \gamma$ with $\gamma \in\{\alpha, \beta\}$. By definition of a derived well-tagged extended process w.r.t $\prec$ and $\mathrm{col}$, one of the following condition is satisfied:

1) There exist $v$ and $i=\operatorname{col}(x)$ such that $u=[v]_{i} \sigma$, $\sigma \vDash \operatorname{test}_{i}\left([v]_{i}\right)$, and $z \prec x$ for any $z \in f v(v)$. Since $x$ is minimal by $\prec$ then $f v(v)=\emptyset$. Hence $u=[v]_{i}$. Thus we deduce that $F c t_{\gamma}(u \downarrow)=\emptyset$. Hence there is a contradiction with $t \in F c t_{\gamma}(u \downarrow)$ and so this condition cannot be satisfied.

2) There exists $M$ such that $f v(M) \subseteq \operatorname{dom}(\Phi) \cap\{z \mid z \prec$ $x\}, f n(M) \cap \mathcal{E}=\emptyset$, and $M \Phi=u$. Thus, we have that $M \Phi \downarrow=u \downarrow$ and so the result holds.

Inductive case $u=x \sigma$ or $u=x \Phi$. Assume $t \in F c t_{\gamma}(u \downarrow)$ and $\operatorname{col}(x) \in \gamma$. By definition of a derived well-tagged extended process w.r.t $\prec$ and $\mathrm{col}$, one of the following condition is satisfied:

1) There exist $v$ and $i=\operatorname{col}(x) \in \gamma$ such that $u=[v]_{i} \sigma$, $\sigma \vDash \operatorname{test}_{i}\left([v]_{i}\right)$, and for all $z \in f v(v), z \prec x$ and either $\operatorname{col}(z) \in \gamma$ or there exists $j$ such that $z=z_{j}^{\gamma^{\prime}}$. Since 
$u=[v]_{i} \sigma$ and $\sigma \vDash \operatorname{test}_{i}\left([v]_{i}\right)$, we can apply Lemma 46 to $v$ and $\sigma \downarrow$. Thus we have that $t \in F c t_{\gamma}\left([v]_{i}(\sigma \downarrow)\right.$. In such a case, it means that there exists $z \in f v(v)$ with $z \prec x$ such that $t \in F_{c t}(z \sigma \downarrow)$ and one of the two conditions is satisfied:

- $\operatorname{col}(z) \in \gamma:$ In such a case, we can apply our inductive hypothesis on $t$ and $z$ and so the result holds.

- there exists $j$ such that $z=z_{j}^{\gamma^{\prime}}$ : Otherwise, we know by hypothesis that $z^{\gamma^{\prime}} \sigma \downarrow \in \mathcal{N}$ or $F c t_{\gamma}\left(z^{\gamma^{\prime}} \sigma \downarrow\right)=\left\{z^{\gamma^{\prime}} \sigma \downarrow\right\}$. Since $t \in F c t_{\gamma}(z \sigma \downarrow)$, we deduce that $z^{\gamma^{\prime}} \sigma \downarrow \notin \mathcal{N}$ and so $F c t_{\gamma}\left(z^{\gamma^{\prime}} \sigma \downarrow\right)=$ $\left\{z^{\gamma^{\prime}} \sigma \downarrow\right\}$. But this implies that $t=z \sigma \downarrow$. Hence the result holds.

2) There exists $M$ such that $f v(M) \subseteq \operatorname{dom}(\Phi) \cap\{z \mid z \prec$ $x\}, f n(M) \cap \mathcal{E}=\emptyset$, and $M \Phi=u$. Thus, we have that $M \Phi \downarrow=u \downarrow$, and we have that $t \in F_{c} t_{\gamma}(M \Phi \downarrow)$.

This allows us to conclude.

Lemma 50: Let $(\mathcal{E} ; \mathcal{P} ; \Phi ; \sigma)$ be a derived well-tagged extended process. Let $M$ be a term such that $f n(M) \cap \mathcal{E}=\emptyset$ and $f v(M) \subseteq \operatorname{dom}(\Phi)$. Let $\mathrm{f}\left(t_{1}, \ldots, t_{m}\right) \in \operatorname{Flawed}(M \Phi \downarrow)$. There exists $M_{1}, \ldots, M_{m}$ such that $f v\left(M_{k}\right) \subseteq \operatorname{dom}(\Phi)$, $f n\left(M_{k}\right) \cap \mathcal{E}=\emptyset, M_{k} \Phi \downarrow=t_{k}$, and $\mu\left(M_{k}\right)<\mu(M)$, for all $k \in\{1, \ldots, m\}$.

Proof: We prove this result by induction on $\mu(M)$.

Base case $\mu(M)=(j, 1)$ : In this case, either we have that $M \in \mathcal{N}$ or $M=w_{j}$. If $M \in \mathcal{N}$, then we have $M \Phi \downarrow=M \in$ $\mathcal{N}$ and Flawed $(M \Phi \downarrow)=\emptyset$. Thus the result holds. If $M=w_{j}$ then, by Lemma $48, \mathrm{f}\left(t_{1}, \ldots, t_{m}\right) \in \operatorname{Flawed}\left(w_{j} \Phi \downarrow\right)$ implies that there exists $M^{\prime}$ such that:

- $f v\left(M^{\prime}\right) \subseteq\left\{w_{1}, \ldots, w_{j-1}\right\}$,

- $f n(M) \cap \mathcal{E}=\emptyset$, and

- $\mathrm{f}\left(t_{1}, \ldots, t_{m}\right) \in$ Flawed $\left(M^{\prime} \Phi \downarrow\right)$.

Since $\mu\left(M^{\prime}\right)<\mu(M)$, thanks to our inductive hypothesis, we deduce that there exist $M_{1}, \ldots, M_{m}$ such that for each $k \in\{1, \ldots, m\}$, we have that: $f v\left(M_{k}\right) \subseteq \operatorname{dom}(\Phi), f n\left(M_{k}\right) \cap$ $\mathcal{E}=\emptyset, M_{k} \Phi \downarrow=t_{k}$, and $\mu\left(M_{k}\right)<\mu\left(M^{\prime}\right)<\mu(M)$.

Inductive step $\mu(M)>(j, 1)$ : In such a case, we have that $M=\mathrm{f}\left(M_{1}, \ldots, M_{n}\right)$. Let $t=\mathrm{g}\left(t_{1}, \ldots, t_{m}\right) \in$ Flawed $(M \Phi \downarrow)$. We do a case analysis on $\mathrm{f}$.

Case $\mathrm{f} \in \Sigma_{i} \cup \Sigma_{\mathrm{tag}_{i}}$ for some $i \in\{1, \ldots, p\}$ : In such a case, $M \Phi \downarrow=\mathrm{f}\left(M_{1} \Phi \downarrow, \ldots, M_{n} \Phi \downarrow\right) \downarrow$. By definition, we know that for all $t \in \operatorname{Flawed}(M \Phi \downarrow)$, we have that $\operatorname{root}(t) \notin \Sigma_{i} \cup \Sigma_{\operatorname{tag}_{i}}$. Thus, thanks to Lemma 26, we deduce that

$$
\operatorname{Flawed}(M \Phi \downarrow) \subseteq \operatorname{Flawed}\left(M_{1} \Phi \downarrow\right) \cup \ldots \cup \operatorname{Flawed}\left(M_{n} \Phi \downarrow\right) \text {. }
$$

Since $\mu\left(M_{k}\right)<\mu(M)$ for any $k \in\{1, \ldots, n\}$, thanks to our inductive hypothesis, we know that there exists $M_{1}^{\prime}, \ldots, M_{m}^{\prime}$ such that $f v\left(M_{j}^{\prime}\right) \subseteq \operatorname{dom}(\Phi), f n\left(M_{j}^{\prime}\right) \cap \mathcal{E}=\emptyset, M_{j}^{\prime} \Phi \downarrow=t_{i}$ and $\mu\left(M_{j}^{\prime}\right)<\mu\left(M_{k}\right)<\mu(M)$, for $j \in\{1, \ldots, m\}$. Hence the result holds.

Case $\mathrm{f}=\langle\rangle$ : In such a case, $M \Phi \downarrow=\mathrm{f}\left(M_{1} \Phi \downarrow, M_{2} \Phi \downarrow\right)$. Moreover, we have that $\operatorname{Flawed}(M \Phi \downarrow)=\operatorname{Flawed}\left(M_{1} \Phi \downarrow\right) \cup$
Flawed $\left(M_{2} \Phi \downarrow\right)$. Since $\mu\left(M_{1}\right)<\mu(M), \mu\left(M_{2}\right)<\mu(M)$ and $t \in \operatorname{Flawed}\left(M_{1} \Phi \downarrow\right) \cup \operatorname{Flawed}\left(M_{2} \Phi \downarrow\right)$, we conclude by applying our inductive hypothesis on $M_{1}$ (or $M_{2}$ ).

Case $\mathrm{f} \in\{\mathrm{pk}, \mathrm{vk}\}$ : In this case, $M \Phi \downarrow=\mathrm{f}\left(M_{1} \Phi \downarrow\right)$ and we have that Flawed $(M \Phi \downarrow)=\emptyset$. Hence the result trivially holds.

Case $f \in\{$ senc, aenc, sign $\}$ : In such a case, we have that $M \Phi \downarrow=\mathrm{f}\left(M_{1} \Phi \downarrow, M_{2} \Phi \downarrow\right)$. We need to distinguish whether $\operatorname{root}\left(M_{1} \Phi \downarrow\right)=\operatorname{tag}_{i}$ for some $i \in\{1, \ldots, p\}$ or not.

If $\operatorname{root}\left(M_{1} \Phi \downarrow\right)=\operatorname{tag}_{i}$ for some $i \in\{1, \ldots, p\}$, then there exists $u_{1}$ such that $M_{1} \Phi \downarrow=\operatorname{tag}_{i}\left(u_{1}\right)$. Hence, we have that Flawed $\left(M_{1} \Phi \downarrow\right)=\operatorname{Flawed}\left(u_{1}\right)$. We have also that:

$$
\text { Flawed }(M \Phi \downarrow)=\operatorname{Flawed}\left(u_{1}\right) \cup \operatorname{Flawed}\left(M_{2} \Phi \downarrow\right) \text {. }
$$

We deduce that $t \in \operatorname{Flawed}\left(M_{1} \Phi \downarrow\right)$ or $t \in \operatorname{Flawed}\left(M_{2} \Phi \downarrow\right)$. Since $\mu\left(M_{1}\right)<\mu(M)$ and $\mu\left(M_{2}\right)<\mu(M)$, we conclude by applying our inductive hypothesis on $M_{1}$ or $M_{2}$.

Otherwise $\operatorname{root}\left(M_{1} \Phi \downarrow\right) \notin\left\{\operatorname{tag}_{1}, \ldots, \operatorname{tag}_{p}\right\}$. In such a case, Flawed $(M \Phi \downarrow)=$ Flawed $\left(M_{1} \Phi \downarrow\right) \cup$ Flawed $\left(M_{2} \Phi \downarrow\right) \cup\{M \Phi \downarrow\}$. If $t=M \Phi \downarrow$, we have that $t_{1}=M_{1} \Phi \downarrow, t_{2}=M_{2} \Phi \downarrow$ and $\mu\left(M_{1}\right)<\mu(M), \mu\left(M_{2}\right)<\mu(M)$. Thus the result holds. If $t \in$ Flawed $\left(M_{1} \Phi \downarrow\right) \cup \operatorname{Flawed}\left(M_{2} \Phi \downarrow\right)$, we conclude by applying our inductive hypothesis on $M_{1}$ or $M_{2}$.

Case $\mathrm{f}=\mathrm{h}$ : This case is analogous to the previous one and can be handled similarly.

Case $f \in\{$ sdec, adec, check $\}$ : In such a case, we have to distinguish two cases depending on whether $f$ is reduced in $M \Phi \downarrow$, or not.

If $\mathrm{f}$ is not reduced, i.e. $M \Phi \downarrow=\mathrm{f}\left(M_{1} \Phi \downarrow, M_{2} \Phi \downarrow\right)$, then we have that

Flawed $(M \Phi \downarrow)=\{M \Phi \downarrow\} \cup$ Flawed $\left(M_{1} \Phi \downarrow\right) \cup$ Flawed $\left(M_{2} \Phi \downarrow\right)$.

Thus if $t=M \Phi \downarrow$, we have that $t_{1}=M_{1} \Phi \downarrow, t_{2}=M_{2} \Phi \downarrow$ and $\mu\left(M_{1}\right)<\mu(M), \mu\left(M_{2}\right)<\mu(M)$. Thus the result holds. Otherwise, we have that $t \in \operatorname{Flawed}\left(M_{1} \Phi \downarrow\right)$ or $t \in$ Flawed $\left(M_{2} \Phi \downarrow\right)$. Since $\mu\left(M_{1}\right)<\mu(M), \mu\left(M_{2}\right)<\mu(M)$, we can conclude by applying our inductive hypothesis on $M_{1}$ or $M_{2}$.

If $\mathrm{f}$ is reduced, then we have that $M_{1} \Phi \downarrow=\mathrm{f}^{\prime}\left(u_{1}, u_{2}\right)$ with $M \Phi \downarrow=u_{1}$ and $\mathrm{f}^{\prime} \in\{$ senc, aenc, sign $\}$. If $\operatorname{root}\left(u_{1}\right)=\operatorname{tag}_{i}$ for some $i \in\{1, \ldots, p\}$, then we have that there exists $u_{1}^{\prime}$ such that $u_{1}=\operatorname{tag}_{i}\left(u_{1}^{\prime}\right)$, Flawed $(M \Phi \downarrow)=\operatorname{Flawed}\left(u_{1}^{\prime}\right)$ and Flawed $\left(M_{1} \Phi \downarrow\right)=\operatorname{Flawed}\left(u_{1}^{\prime}\right) \cup \operatorname{Flawed}\left(u_{2}\right)$. Thus, we have that Flawed $(M \Phi \downarrow) \subseteq \operatorname{Flawed}\left(M_{1} \Phi \downarrow\right)$. Otherwise, if $\operatorname{root}\left(u_{1}\right) \notin\left\{\operatorname{tag}_{1}, \ldots, \operatorname{tag}_{p}\right\}$, then we have that

$$
\text { Flawed }\left(M_{1} \Phi \downarrow\right)=\left\{M_{1} \Phi \downarrow\right\} \cup \operatorname{Flawed}\left(u_{1}\right) \cup \operatorname{Flawed}\left(u_{2}\right)
$$

and Flawed $(M \Phi \downarrow)=\operatorname{Flawed}\left(u_{1}\right)$. Thus, we also have that Flawed $(M \Phi \downarrow) \subseteq$ Flawed $\left(M_{1} \Phi \downarrow\right)$. In both cases, we have that Flawed $(M \Phi \downarrow) \subseteq$ Flawed $\left(M_{1} \Phi \downarrow\right)$ and since $\mu\left(M_{1}\right)<\mu(M)$, we can conclude by applying our inductive hypothesis on $M_{1}$.

In the following lemma, we will use the factors of the signature only composed of \langle\rangle , denoted $F c t_{\langle\rangle}$. Typically, for all terms $u$, for all context built only on \langle\rangle , for all terms 
$u_{1}, \ldots, u_{n}$, if $u=C\left[u_{1}, \ldots, u_{n}\right]$ and for all $k \in\{1, \ldots, n\}$, $\operatorname{root}\left(u_{i}\right) \neq\langle\rangle$ then $\left.F_{c t}\right\rangle(u)=\left\{u_{1}, \ldots, u_{n}\right\}$.

Lemma 51: Let $(\mathcal{E} ; \mathcal{P} ; \Phi ; \sigma)$ be a derived well-tagged extended process w.r.t $\prec$ and col. Assume that for all assignment variables $z$, new $\mathcal{E}$. $\Phi \forall z \sigma \downarrow$. Let $M$ such that $f v(M) \subseteq \operatorname{dom}(\Phi), f n(M) \cap \mathcal{E}=\emptyset$. For all $\left\{\gamma, \gamma^{\prime}\right\}=\{\alpha, \beta\}$, for all $t \in F c t_{\gamma}(M \Phi \downarrow)$, if $t \notin F c t_{\langle\rangle}(M \Phi \downarrow)$ and for all assignment variable $z$, for all $w \in \operatorname{dom}(\Phi), z \prec w$ and $\mu(w) \leq \mu(M)$ implies $z \sigma \downarrow \neq t$ then there exists $M^{\prime}$ such that $\mu\left(M^{\prime}\right)<\mu(M), f n(M) \cap \mathcal{E}=\emptyset$ and $t \in F c t_{\langle\rangle}\left(M^{\prime} \Phi \downarrow\right)$.

Proof: We do a proof by induction on $\mu(M)$ :

Base case $\mu(M)=(0,1):$ In this case, we have that $M \in \mathcal{N}$ which means that $M \Phi \downarrow=M \in \mathcal{N}$ and $F c t_{\gamma}(M \Phi \downarrow)=\emptyset$. Thus the result holds.

Base case $\mu(M)=(j, 1)$ : In this case, we have $M=w_{j}$. Let $\left\{\gamma, \gamma^{\prime}\right\}=\{\alpha, \beta\}$. Let $t \in F c t_{\gamma}(M \Phi \downarrow)$ such that $t \notin$ $F_{c t}(M \Phi \downarrow)$. We do a case analysis on $\operatorname{col}\left(w_{j}\right)$ :

Case $\operatorname{col}\left(w_{j}\right) \in \gamma$ : In this case, since for all assignment variable $z$, for all $w \in \operatorname{dom}(\Phi), z \prec w$ and $\mu(w) \leq \mu(M)$ implies $z \sigma \downarrow \neq t$, than we can deduce that for all assignment variables $z \prec w_{j}, z \sigma \downarrow \neq t$. Thus by Lemma 49 , we obtain that there exists $M^{\prime}$ such that $f v\left(M^{\prime}\right) \subseteq \operatorname{dom}(\Phi) \cap\{z \mid z \prec x\}$, $f n\left(M^{\prime}\right) \cap \mathcal{E}=\emptyset$ and $t \in F_{c t}\left(M^{\prime} \Phi \downarrow\right) . f v\left(M^{\prime}\right) \subseteq \operatorname{dom}(\Phi) \cap$ $\{z \mid z \prec x\}$ implies that $\mu\left(M^{\prime}\right)=\left(k, k^{\prime}\right)$ with $k<j$ and so $\mu\left(M^{\prime}\right)<\mu(M)$. If $t \in F c t_{\langle\rangle}\left(M^{\prime} \Phi \downarrow\right)$ then the result holds. Otherwise, we can apply our inductive hypothesis on $t$ and $M^{\prime}$ and so the result holds.

Case $\operatorname{col}\left(w_{j}\right) \in \gamma^{\prime}:$ Since $t \notin F c t_{\langle\rangle}(M \Phi \downarrow)$, we deduce that there exists $u \in F c t_{\langle\rangle}(M \Phi \downarrow)$ s.t. $\operatorname{tagroot}(u)=\gamma$ and $t \in F c t_{\gamma}(u)$. Note that $\operatorname{tagroot}(u) \notin \gamma^{\prime} \cup\{0\}$ otherwise it would contradict the fact that $t \in F c t_{\gamma}(M \Phi \downarrow)$. But $u \in F c t_{\gamma^{\prime}}(M \Phi \downarrow)$. Moreover, $u \in F c t_{\langle\rangle}(M \Phi \downarrow)$ implies that $u$ is deducible in new $\mathcal{E} . \Phi$. Thus we deduce that for all assignment variables $z, z \sigma \downarrow \neq u$. By applying the same proof as case $\operatorname{col}\left(w_{j}\right) \in \gamma$, we deduce that there exists $M^{\prime}$ such that $f n\left(M^{\prime}\right) \cap \mathcal{E}=\emptyset, \mu\left(M^{\prime}\right)<\mu(M)$ and $u \in F_{c t} t_{\langle\rangle}\left(M^{\prime} \Phi \downarrow\right)$. But $t \in F_{c t}(u)$, tagroot $(u)=\gamma$ and $u \in F c t_{\langle\rangle}\left(M^{\prime} \Phi \downarrow\right)$ implies that $t \in F c t_{\gamma}\left(M^{\prime} \Phi \downarrow\right)$ and $t \notin F c t_{\langle\rangle}\left(M^{\prime} \Phi \downarrow\right)$. Hence we can apply our inductive hypothesis on $M^{\prime}$ and $t$ which allows us to conclude.

Inductive step $\mu(M)>(j, 1)$ : In such a case, we have that $M=\mathrm{f}\left(M_{1}, \ldots, M_{n}\right)$. Let $t \in F c t_{\gamma}(M \Phi \downarrow)$ such that $t \notin$ $F c t_{\langle\rangle}(M \Phi \downarrow)$. We do a case analysis on $\mathrm{f}$.

Case $\mathrm{f} \in \Sigma_{i} \cup \Sigma_{\mathrm{tag}_{i}}$ for some $i \in \gamma$ : In such a case, $M \Phi \downarrow=$ $\mathrm{f}\left(M_{1} \Phi \downarrow, \ldots, M_{n} \Phi \downarrow\right) \downarrow$. By definition, we know that for all $t \in F_{c} t_{\gamma}(M \Phi \downarrow)$, we have that $\operatorname{root}(t) \notin \Sigma_{i} \cup \Sigma_{\operatorname{tag}_{i}}$. Thus, thanks to Lemma 46, we deduce that there exists

$$
F c t_{\gamma}(M \Phi \downarrow) \subseteq F c t_{\gamma}\left(M_{1} \Phi \downarrow\right) \cup \ldots \cup F c t_{\gamma}\left(M_{n} \Phi \downarrow\right) .
$$

Thus there exists $k \in\{1, \ldots, n\}$ such that $t \in F_{c t}\left(M_{k} \Phi \downarrow\right)$. If $t \in \operatorname{Fct}_{\langle\rangle}\left(M_{k} \Phi \downarrow\right)$ then the result holds, else we apply our inductive hypothesis on $t$ and $M_{k}$ and so the result also holds.

Case $\mathrm{f} \in \Sigma_{i} \cup \Sigma_{\operatorname{tag}_{i}}$ for some $i \notin \gamma$ : In such a case, $M \Phi \downarrow=$ $\mathrm{f}\left(M_{1} \Phi \downarrow, \ldots, M_{n} \Phi \downarrow\right) \downarrow$. We assumed that $t \notin F c t_{\langle\rangle}(M \Phi \downarrow)$ hence there exists $u \in F c t_{\langle\rangle}(M \Phi \downarrow)$ s.t. $\operatorname{tagroot}(u)=\gamma$ and $t \in F_{c} t_{\gamma}(u)$. But it also implies that tagroot $(M \Phi \downarrow) \in \gamma \cup\{0\}$. Hence, by applying Lemma 26 , we deduce that there exists $k \in\{1, \ldots, n\}$ such that $M \Phi \downarrow \in \operatorname{st}\left(M_{k} \Phi \downarrow\right)$. Moreover, it also implies that $u \in F c t_{\gamma}^{\prime}\left(M_{k} \Phi \downarrow\right)$.

If $u \in F c t_{\langle\rangle}\left(M_{k} \Phi \downarrow\right)$ then we deduce that $\operatorname{root}\left(M_{k} \Phi \downarrow\right) \notin$ $\gamma^{\prime}$ and so, by Lemma 26, $M_{k} \Phi \downarrow=M \Phi \downarrow$. Since we had $t \notin F c t_{\langle\rangle}(M \Phi \downarrow)$, then we also have $t \notin F c t_{\langle\rangle}\left(M_{k} \Phi \downarrow\right)$ and so we conclude by applying our inductive hypothesis on $t$ and $M_{k}$.

if $u \notin F c t_{\langle\rangle}\left(M_{k} \Phi \downarrow\right)$ then we can apply our inductive hypothesis on $u, \gamma^{\prime}$ and $M_{k}$. Indeed, since $u \in F c t_{\langle\rangle}(M \Phi \downarrow)$, then $u$ is deducible in new $\mathcal{E} . \Phi$ and so we deduce that for all assignment variable $z, z \sigma \downarrow \neq u$. Hence we obtain that there exists $M^{\prime}$ such that $\mu\left(M^{\prime}\right)<\mu\left(M_{k}\right), f n(M) \cap$ $\mathcal{E}=\emptyset$ and $u \in F_{c t}\left(M^{\prime} \Phi \downarrow\right)$. But $t \in F c t_{\gamma}(u)$ and $u \in F c t_{\langle\rangle}\left(M^{\prime} \Phi \downarrow\right)$. Hence we deduce that $t \in F c t_{\gamma}\left(M^{\prime} \Phi \downarrow\right)$ and $t \notin F c t_{\langle\rangle}\left(M^{\prime} \Phi \downarrow\right)$. We conclude by applying once again our inductive hypothesis but on $t, \gamma$ and $M^{\prime}$.

Case $\mathrm{f}=\langle\rangle$ : In such a case, $M \Phi \downarrow=\mathrm{f}\left(M_{1} \Phi \downarrow, M_{2} \Phi \downarrow\right)$. Moreover, we have that $F c t_{\gamma}(M \Phi \downarrow)=F c t_{\gamma}\left(M_{1} \Phi \downarrow\right) \cup$ $F_{c t}\left(M_{2} \Phi \downarrow\right)$. Since $\mu\left(M_{1}\right)<\mu(M), \mu\left(M_{2}\right)<\mu(M)$ and $t \in F c t_{\gamma}\left(M_{1} \Phi \downarrow\right) \cup F c t_{\gamma}\left(M_{2} \Phi \downarrow\right)$, we conclude by applying our inductive hypothesis on $t$ and $M_{1}$ (or $M_{2}$ ).

Case $\mathrm{f} \in\{\mathrm{pk}, \mathrm{vk}\}:$ In this case, $M \Phi \downarrow=\mathrm{f}\left(M_{1} \Phi \downarrow\right)$ and we have that $F c t_{\gamma} M \Phi \downarrow=\emptyset$. Hence the result trivially holds.

Case $f \in\{$ senc, aenc, sign $\}$ : In such a case, we have that $M \Phi \downarrow=\mathrm{f}\left(M_{1} \Phi \downarrow, M_{2} \Phi \downarrow\right)$. We need to distinguish whether $\operatorname{root}\left(M_{1} \Phi \downarrow\right)=\operatorname{tag}_{i}$ for some $i \in\{1, \ldots, p\}$ or not.

If $\operatorname{root}\left(M_{1} \Phi \downarrow\right)=\operatorname{tag}_{i}$ for some $i \in\{1, \ldots, p\}$, then there exists $u_{1}$ such that $M_{1} \Phi \downarrow=\operatorname{tag}_{i}\left(u_{1}\right)$. Assume first that $i \in \gamma^{\prime}$. In such a case $F c t_{\gamma}(M \Phi \downarrow)=\left\{F_{c t}(M \Phi \downarrow)\right\}$ and $F c t_{\langle\rangle}(M \Phi \downarrow)=\left\{F_{c t}(M \Phi \downarrow)\right\}$. Hence it contradicts the fact that $t \notin F c t_{\langle\rangle}(M \Phi \downarrow)$. We can thus deduce that $i \in \gamma$. But in such a case, we have that $F c t_{\gamma}\left(M_{1} \Phi \downarrow\right)=F c t_{\gamma}\left(u_{1}\right)$ and:

$$
F c t_{\gamma}(M \Phi \downarrow)=F c t_{\gamma}\left(u_{1}\right) \cup F c t_{\gamma}\left(M_{2} \Phi \downarrow\right) .
$$

We deduce that $t \in F c t_{\gamma}\left(M_{1} \Phi \downarrow\right)$ or $t \in F c t_{\gamma}\left(M_{2} \Phi \downarrow\right)$. Since $\mu\left(M_{1}\right)<\mu(M)$ and $\mu\left(M_{2}\right)<\mu(M)$, we conclude by applying our inductive hypothesis on $M_{1}$ or $M_{2}$.

Otherwise $\operatorname{root}\left(M_{1} \Phi \downarrow\right) \notin\left\{\operatorname{tag}_{1}, \ldots, \operatorname{tag}_{p}\right\}$. In such a case, $F c t_{\gamma}(M \Phi \downarrow)=\{M \Phi \downarrow\}$ and $F c t_{\langle\rangle}(M \Phi \downarrow)=\{M \Phi \downarrow\}$. But we assume that $t \notin F c t_{\langle\rangle}(M \Phi \downarrow)$ hence this case is impossible.

Case $\mathrm{f}=\mathrm{h}$ : This case is analogous to the previous one and can be handled similarly.

Case $f \in\{\mathrm{sdec}$, adec, check\}: In such a case, we have to distinguish two cases depending on whether $f$ is reduced in $M \Phi \downarrow$, or not.

If $\mathrm{f}$ is not reduced, i.e. $M \Phi \downarrow=\mathrm{f}\left(M_{1} \Phi \downarrow, M_{2} \Phi \downarrow\right)$, then we have that

$$
\operatorname{Fct}_{\gamma}(M \Phi \downarrow)=\{M \Phi \downarrow\} .
$$

Once again this is in contradiction with our hypothesis that $t \notin F c t_{\langle\rangle}(M \Phi \downarrow)$. 
We now focus on the case where $f$ is reduced: we have that $M_{1} \Phi \downarrow=\mathrm{f}^{\prime}\left(u_{1}, u_{2}\right)$ with $M \Phi \downarrow=u_{1}$ and $\mathrm{f}^{\prime} \in$ \{senc, aenc, sign\}. We have to do a case analysis on $\operatorname{root}\left(u_{1}\right)$ :

- if $\operatorname{root}\left(u_{1}\right)=\operatorname{tag}_{i}$ for some $i \in \gamma$. In such a case, there exists $u_{1}^{\prime}$ such that $u_{1}=\operatorname{tag}_{i}\left(u_{1}^{\prime}\right), F c t_{\gamma}(M \Phi \downarrow)=$ $F c t_{\gamma}\left(u_{1}^{\prime}\right)$ and $F_{c t}\left(M_{1} \Phi \downarrow\right)=F_{c t}\left(u_{1}^{\prime}\right) \cup F c t_{\gamma}\left(u_{2}\right)$. Thus we deduce that $F c t_{\gamma}(M \Phi \downarrow) \subseteq F_{c t}\left(M_{1} \Phi \downarrow\right)$. We can conclude thanks to our inductive hypothesis on $t$ and $M_{1}$.

- if $\operatorname{root}\left(u_{1}\right)=\operatorname{tag}_{i}$ for some $i \notin \gamma$. In such a case, $F c t_{\gamma}(M \Phi \downarrow)=\{M \Phi \downarrow)$ which contradicts the hypothesis $t \notin F c t_{\langle\rangle}(M \Phi \downarrow)$.

- otherwise, $\operatorname{root}\left(u_{1}\right) \notin\left\{\operatorname{tag}_{1}, \ldots, \operatorname{tag}_{p}\right\}$, then we have that $\mathrm{f}^{\prime}\left(u_{1}, u_{2}\right) \in \operatorname{Flawed}\left(M_{1} \Phi \downarrow\right)$. By Lemma 50, we deduce that there exists $M^{\prime}$ such that $\mu\left(M^{\prime}\right)<\mu\left(M_{1}\right)$, $f n\left(M^{\prime}\right) \cap \mathcal{E}=\emptyset$ and $M^{\prime} \Phi \downarrow=u_{1}$. Since $u_{1}=M \Phi \downarrow$ and $\mu\left(M^{\prime}\right)<\mu(M)$ then we can apply our inductive hypothesis on $t, \alpha$ and $M^{\prime}$ and so the result holds.

Lemma 52: Let $A=(\mathcal{E} ; \mathcal{P} ; \Phi ; \sigma)$ be a derived well-tagged process, and let $\left(\rho_{\alpha}, \rho_{\beta}\right)$ be compatible with $A$. Let $u$ be a ground term in normal form that do not use names in $\mathcal{E}_{\alpha} \uplus$ $\mathcal{E}_{\beta}$. We have that there exists a context $C$ (possibly a hole) built only using \langle\rangle , and terms $u_{1}, \ldots, u_{m}$ such that $u=$ $C\left[u_{1}, \ldots, u_{m}\right]$, and for all $i \in\{1, \ldots, m\}$,

- either $u_{i} \in \operatorname{Flawed}(u)$;

- or $u_{i} \in$ Fct $_{\Sigma_{0}}(u)$ and $\delta_{\alpha}\left(u_{i}\right)=\delta_{\beta}\left(u_{i}\right)$,

- or $u_{i}=\mathrm{f}(n)$ for some $\mathrm{f} \in\{\mathrm{pk}, \mathrm{vk}\}$ and $n \in \mathcal{N}$,

- or $u_{i} \in \operatorname{dom}\left(\rho_{\alpha}^{+}\right) \cup \operatorname{dom}\left(\rho_{\beta}^{+}\right)$.

Proof: Let $u$ a ground term in normal form and let $\left\{v_{1}, \ldots, v_{n}\right\}=F c t_{\Sigma_{0}}(u)$. Thus there exists a context $D$ (possibly a hole) built on $\Sigma_{0}$ such that $u=D\left[v_{1}, \ldots, v_{n}\right]$. We now prove the result by induction on $|D|$.

Base case $|D|=0$ : We show that the result holds and in such a case the context $C$ is reduced to a hole. Since $|D|=0$, we know that $F c t_{\Sigma_{0}}(u)=u$ and so either $\operatorname{tagroot}(u)=i$ with $i \in\{1, \ldots, p\}$ or $\operatorname{tagroot}(u)=\perp$. If $u \in \operatorname{dom}\left(\rho_{\alpha}^{+}\right) \cup$ $\operatorname{dom}\left(\rho_{\beta}^{+}\right)$, then the result trivially holds. Otherwise, we have that $\delta_{\alpha}(u)=\delta_{\beta}(u)$ by definition of $\delta_{\alpha}$ and $\delta_{\beta}$. Hence the result holds.

Inductive step $|D|>0$ : There exists $\mathrm{f} \in \Sigma_{0}$, and $v_{1}, \ldots, v_{k}$ such that $u=\mathrm{f}\left(u_{1}, \ldots, u_{k}\right)$. We do a case analysis on $\mathrm{f}$.

Case $\mathrm{f}=\langle\rangle$ : In such a case, there exist two contexts $D_{1}, D_{2}$ (possibly holes) built on $\Sigma_{0}$ such that:

- $D=\left\langle D_{1}, D_{2}\right\rangle$ with $\left|D_{1}\right|,\left|D_{2}\right|<|D|$,

- $u_{1}=D_{1}\left[v_{1}^{1}, \ldots, v_{n_{1}}^{1}\right]$ and $\left\{v_{1}^{1}, \ldots, v_{n_{1}}^{1}\right\}=\operatorname{Fct}_{\Sigma_{0}}\left(u_{1}\right)$,

- $u_{2}=D_{1}\left[v_{1}^{2}, \ldots, v_{n_{1}}^{2}\right]$ and $\left\{v_{1}^{2}, \ldots, v_{n_{2}}^{2}\right\}=$ Fct $_{\Sigma_{0}}\left(u_{2}\right)$

By applying our inductive hypothesis on $u_{1}$ and $u_{2}$, we know that there exist two contexts $C_{1}$ and $C_{2}$. Since

- Flawed $(u)=\operatorname{Flawed}\left(u_{1}\right) \cup \operatorname{Flawed}\left(u_{2}\right)$, and

- Fct $_{\Sigma_{0}}(u)=$ Fct $_{\Sigma_{0}}\left(u_{1}\right) \uplus$ Fct $_{\Sigma_{0}}\left(u_{2}\right)$,

we conclude that $C=\left\langle C_{1}, C_{2}\right\rangle$ satisfies all the conditions stated in the lemma.
Case $\mathrm{f} \in\{\mathrm{pk}, \mathrm{vk}\}$ and $u=\mathrm{f}(n)$ for some $n \in N$ : The result trivially hold by choosing the context $C$ to be a hole.

Otherwise, we have that

$$
\text { Flawed }(u)=\{u\} \cup \operatorname{Flawed}\left(u_{1}\right) \cup \ldots \cup \operatorname{Flawed}\left(u_{k}\right) \text {. }
$$

Since $u \in \operatorname{Flawed}(u)$, we can choose $C$ to be the context reduced to a hole. The result trivially holds.

Lemma 53: Let $A=(\mathcal{E} ; \mathcal{P} ; \Phi ; \sigma)$ be a derived well-tagged extended process, and let $\left(\rho_{\alpha}, \rho_{\beta}\right)$ be compatible with $A$. Let $M$ be a term such that $f v(M) \subseteq \operatorname{dom}(\Phi)$ and $f n(M) \cap \mathcal{E}=\emptyset$. We assume that $\mathcal{E}=\mathcal{E}_{0} \uplus \mathcal{E}_{\alpha} \uplus \mathcal{E}_{\beta}, f n(\Phi) \cap\left(\mathcal{E}_{\alpha} \uplus \mathcal{E}_{\beta}\right)=\emptyset$, and one of the two following conditions is satisfied:

1) new $\mathcal{E} . \Phi \not k$ for any $k \in K_{S}$; or

2) new $\mathcal{E} . \delta(\Phi \downarrow) \not \forall k$ for any $k \in \delta_{\alpha}\left(K_{S}\right) \cup \delta_{\beta}\left(K_{S}\right)$.

with $K_{S}=\left\{t, \operatorname{pk}(t), \operatorname{vk}(t) \mid t\right.$ ground, $t \in \operatorname{dom}\left(\rho_{\alpha}^{+}\right) \cup$ $\left.\operatorname{dom}\left(\rho_{\beta}^{+}\right)\right\}$. We have that $\delta_{\gamma}(M \Phi \downarrow)=M \delta(\Phi \downarrow) \downarrow$ with $\gamma \in$ $\{\alpha, \beta\}$.

Proof: Let $\Phi \downarrow=\left\{w_{1} \triangleright u_{1}, \ldots, w_{n} \triangleright u_{n}\right\}$. We prove this result by induction on $\mu(M)$ :

Base case $\mu(M)=(0,0)$ : There exists no term $M$ such that $|M|=0$, thus the result holds.

Inductive step $\mu(M)>(0,0)$ : We first prove there exists $\gamma \in$ $\{\alpha, \beta\}$ such that $\delta_{\gamma}(M \Phi \downarrow)=M \delta(\Phi \downarrow) \downarrow$ and then we show that $\delta_{\alpha}(M \Phi \downarrow)=\delta_{\beta}(M \Phi \downarrow)$.

Assume first that $|M|=1$, i.e. either $M \in \mathcal{N}$ or there exists $j \in\{1, \ldots, n\}$ such that $M=w_{j}$.

Case $M \in \mathcal{N}$. In such a case, we have that $M \Phi \downarrow=M$, and $M \notin \mathcal{E}$. Hence, we have that new $\mathcal{E} . \Phi \vdash M$ and also that new $\mathcal{E} . \delta(\Phi \downarrow) \vdash M$. In case condition 1 is satisfied, we easily deduce that $M \notin K_{S}$. Otherwise, we know that the condition 2 is satisfied, and thus $M \notin \delta_{\alpha}\left(K_{S}\right) \cup \delta_{\beta}\left(K_{S}\right)$. Again, we want to conclude that $M \notin K_{S}$. Assume that this is not the case, i.e. $M \in K_{S}$. This means that $M$ is a name in $\operatorname{dom}\left(\rho_{\alpha}^{+}\right)$ (or $\operatorname{dom}\left(\rho_{\beta}^{+}\right)$). Hence, we have that $\delta_{\beta}(M) \in \delta_{\beta}\left(K_{S}\right)$, and $\delta_{\beta}(M)=M$. Hence, we deduce that $M \in \delta_{\beta}\left(K_{S}\right)$, and this leads to a contradiction, since in such a case, by hypothesis $M$ can not be deducible from new $\mathcal{E} . \delta(\Phi \downarrow)$. Thus, in any case, we have that $M \notin K_{S}$, and thus $M \notin \operatorname{dom}\left(\rho_{\alpha}^{+}\right) \cup \operatorname{dom}\left(\rho_{\beta}^{+}\right)$. Hence, we have that $\delta_{\gamma}(M \Phi \downarrow)=\delta_{\gamma}(M)=M=M \delta(\Phi \downarrow) \downarrow$ for any $\gamma \in\{\alpha, \beta\}$.

Case $M=w_{j}$ for some $j \in\{1, \ldots, n\}$. We know that $w_{j}$ is colored with $\gamma \in\{\alpha, \beta\}$. Hence, we have that $w_{j} \delta(\Phi \downarrow)=$ $\delta_{\gamma}\left(w_{j} \Phi \downarrow\right)$. Since $u_{j}$ is in normal form, then by Lemma 37, we know that $\delta_{\gamma}\left(w_{j} \Phi\right)$ is also in normal form. Thus, we have that $\delta_{\gamma}(M \Phi \downarrow)=M \delta(\Phi \downarrow) \downarrow$.

Otherwise, if $|M|>1$, then there exists a symbol $f$ and $M_{1}, \ldots, M_{n}$ such that $M=\mathrm{f}\left(M_{1}, \ldots, M_{n}\right)$. We do a case analysis on $\mathrm{f}$.

Case $f \in \Sigma_{i} \cup \Sigma_{\operatorname{tag}_{i}}$ with $i \in\{1, \ldots, p\}$. Consider $\gamma \in\{\alpha, \beta\}$ such that $i \in \gamma$. In such a case, let $t=$ $\mathrm{f}\left(M_{1} \Phi \downarrow, \ldots, M_{n} \Phi \downarrow\right)$. Since $\mathrm{f} \in \Sigma_{i}$ (resp. $\left.\Sigma_{\operatorname{tag}_{i}}\right)$, then there exists a context $C$ built upon $\Sigma_{i}$ (resp. $\Sigma_{\operatorname{tag}_{i}}$ ) such that $t=C\left[u_{1}, \ldots, u_{m}\right]$ and $u_{1}, \ldots, u_{m}$ are factor of $t$ in normal 
form. By Lemma 26, we know that there exists a context $D$ (possibly a hole) over $\Sigma_{i}$ (resp. $\Sigma_{\operatorname{tag}_{i}}$ ) such that $t \downarrow=D\left[u_{i_{1}}, \ldots, u_{i_{k}}\right]$ with $i_{1}, \ldots, i_{k} \in\{0, \ldots, m\}$ and $u_{0}=n_{\text {min }}$. But thanks to Lemma 27, 35 and 37, we also that $C\left[\delta_{\gamma}\left(u_{1}\right), \ldots, \delta_{\gamma}\left(u_{m}\right)\right] \downarrow=D\left[\delta_{\gamma}\left(u_{i_{1}}\right), \ldots, \delta_{\gamma}\left(u_{i_{k}}\right)\right]$. But $C$ and $D$ are both built on $\Sigma_{i}$ (resp. $\Sigma_{\operatorname{tag}_{i}}$ ), thus by definition of $\delta_{\gamma}$, we have that $\delta_{\gamma}(t) \downarrow=C\left[\delta_{\gamma}\left(u_{1}\right), \ldots, \delta_{\gamma}\left(u_{m}\right)\right] \downarrow$ and $\delta_{\gamma}(t \downarrow)=D\left[\delta_{\gamma}\left(u_{i_{1}}\right), \ldots, \delta_{\gamma}\left(u_{i_{k}}\right)\right]$. Hence, the equality, $\delta_{\gamma}(t \downarrow)=\delta_{\gamma}(t) \downarrow$, holds. But $t \downarrow=M \Phi \downarrow$ which means that $\delta_{\gamma}(M \Phi \downarrow)=\delta_{\gamma}(t) \downarrow$. We have that:

$$
\begin{aligned}
\delta_{\gamma}(t) \downarrow & =\delta_{\gamma}\left(\mathrm{f}\left(M_{1} \Phi \downarrow, \ldots, M_{n} \Phi \downarrow\right)\right) \downarrow \\
& =\mathrm{f}\left(\delta_{\gamma}\left(M_{1} \Phi \downarrow\right), \ldots, \delta_{\gamma}\left(M_{n} \Phi \downarrow\right)\right) \downarrow
\end{aligned}
$$

Since $\mu\left(M_{1}\right)<\mu(M), \ldots, \mu\left(M_{n}\right)<\mu(M)$, we can apply our inductive hypothesis on $M_{1}, \ldots, M_{n}$. This gives us $\delta_{\gamma}(t) \downarrow=$ $\mathrm{f}\left(M_{1} \delta(\Phi \downarrow) \downarrow, \ldots, M_{n} \delta(\Phi \downarrow) \downarrow\right) \downarrow=\mathrm{f}\left(M_{1}, \ldots, M_{n}\right) \delta(\Phi \downarrow) \downarrow$. Thus we can conclude that $\delta_{\gamma}(M \Phi \downarrow)=\delta_{\gamma}(t) \downarrow=M \delta(\Phi \downarrow) \downarrow$.

Case $f \in \Sigma_{0} \backslash\{$ sdec, adec, check $\}$ : In this case, we have that $M \Phi \downarrow=\mathrm{f}\left(M_{1} \Phi \downarrow, \ldots, M_{n} \Phi \downarrow\right)$. By applying our inductive hypothesis on $M_{1}, \ldots, M_{n}$, we have that

$$
\delta_{\alpha}\left(M_{k} \Phi \downarrow\right)=\delta_{\beta}\left(M_{k} \Phi \downarrow\right), \text { for all } k \in\{1, \ldots, n\} .
$$

Thus we have that $\delta_{\gamma}(M \Phi \downarrow)$ = $\mathrm{f}\left(\delta_{\gamma^{\prime}}\left(M_{1} \Phi \downarrow\right), \ldots, \delta_{\gamma^{\prime}}\left(M_{n} \Phi \downarrow\right)\right)$ with $\gamma, \gamma^{\prime} \in\{\alpha, \beta\}$. Applying our inductive hypothesis on $M_{1}, \ldots, M_{n}$, we deduce that

$$
\delta_{\gamma}(M \Phi \downarrow)=\mathrm{f}\left(M_{1} \delta(\Phi \downarrow) \downarrow, \ldots, M_{n} \delta(\Phi \downarrow) \downarrow\right)=M \delta(\Phi \downarrow) \downarrow .
$$

Case $f \in\{$ sdec, adec, check $\}$ : If we first assume that the root occurence $\mathrm{f}$ is not reduced in $M \Phi \downarrow$ then the proof is similar to the previous case. Thus, we focus on the case where the root occurence of $f$ is reduced, and we consider the case where $\mathrm{f}=$ sdec. The other cases can be done in a similar way. In such a situation, we know that there exist $v_{1}, v_{2}$ such that $M_{1} \Phi \downarrow=$ $\operatorname{senc}\left(v_{1}, v_{2}\right), M_{2} \Phi \downarrow=v_{2}$ and $M \Phi \downarrow=v_{1}$. According to the definition of $\delta_{\gamma}$, we know that there exists $\gamma \in\{\alpha, \beta\}$ such that $\delta_{\gamma}\left(\operatorname{senc}\left(v_{1}, v_{2}\right)\right)=\operatorname{senc}\left(\delta_{\gamma}\left(v_{1}\right), \delta_{\gamma}\left(v_{2}\right)\right)$. For such $\gamma$, we have that $\operatorname{sdec}\left(\delta_{\gamma}\left(M_{1} \Phi \downarrow\right), \delta_{\gamma}\left(M_{2} \Phi \downarrow\right)\right) \downarrow=\delta_{\gamma}(M \Phi \downarrow)$. But by applying our inductive hypothesis on $M_{1}$ and $M_{2}$, we obtain $\delta_{\gamma}(M \Phi \downarrow)=\operatorname{sdec}\left(M_{1} \delta(\Phi \downarrow) \downarrow, M_{2} \delta(\Phi \downarrow) \downarrow\right) \downarrow=M \delta(\Phi \downarrow) \downarrow$.

It remains to prove that $\delta_{\alpha}(M \Phi \downarrow)=\delta_{\beta}(M \Phi \downarrow)$. We have shown that there exists $\gamma_{0} \in\{\alpha, \beta\}$ such that $\delta_{\gamma_{0}}(M \Phi \downarrow)=$ $M \delta(\Phi \downarrow) \downarrow$. Thanks to Lemma 52, we know that there exists a context $C$ built over $\{\langle\rangle\}$, and $v_{1}, \ldots, v_{m}$ terms such that $M \Phi \downarrow=C\left[v_{1}, \ldots, v_{m}\right]$ and for all $i \in\{1, \ldots, m\}$ :

- either $v_{i} \in \operatorname{Flawed}(M \Phi \downarrow)$

- or $v_{i} \in F c t_{\Sigma_{0}}(M \Phi \downarrow)$ and $\delta_{\alpha}\left(v_{i}\right)=\delta_{\beta}\left(v_{i}\right)$.

- or $v_{i}=\mathrm{f}(n)$ for some $\mathrm{f} \in\{\mathrm{pk}, \mathrm{vk}\}$ and $n \in \mathcal{N}$,

- or $v_{i} \in \operatorname{dom}\left(\rho_{\alpha}^{+}\right) \cup \operatorname{dom}\left(\rho_{\beta}^{+}\right)$.

Note that $C$ being built upon $\{\langle\rangle\}$ means that $v_{i}$ is deducible in new $\mathcal{E} . \Phi$ for all $i \in\{1, \ldots, m\}$. Furthermore, since $C\left[v_{1}, \ldots, v_{m}\right]$ is in normal form,

$$
\delta_{\gamma_{0}}(M \Phi \downarrow)=C\left[\delta_{\gamma_{0}}\left(v_{1}\right), \ldots, \delta_{\gamma_{0}}\left(v_{m}\right)\right] .
$$

But we have shown that $\delta_{\gamma_{0}}(M \Phi \downarrow)=M \delta(\Phi \downarrow) \downarrow$, thus $\delta_{\gamma_{0}}\left(v_{i}\right)$ is deducible from $\delta(\Phi \downarrow)$, for all $i \in\{1, \ldots, m\}$. Now, we distinguish several cases depending on which condition is fullfilled by $v_{i}$.

Case $v_{i} \in \operatorname{Flawed}(M \Phi \downarrow)$ : There exists $w_{1}, \ldots, w_{\ell}$ terms and a function symbol $\mathrm{f}$ such that $v_{i}=\mathrm{f}\left(w_{1}, \ldots, w_{\ell}\right)$. By Lemma 50, there exists $N_{1}, \ldots, N_{\ell}$ such that for all $k \in\{1, \ldots, \ell\}, \mu\left(N_{k}\right)<\mu(M)$ and $N_{k} \Phi \downarrow=w_{k}$. Hence, by applying inductive hypothesis on $N_{1}, \ldots, N_{\ell}$, we obtain that $\delta_{\alpha}\left(N_{k} \Phi \downarrow\right)=\delta_{\beta}\left(N_{k} \Phi \downarrow\right)$, for all $k \in\{1, \ldots, \ell\}$. Thus, thanks to $v_{i}$ being in normal form, we can conclude that $\delta_{\alpha}\left(v_{i}\right)=\delta_{\beta}\left(v_{i}\right)$.

Case $v_{i} \in$ Fct $_{\Sigma_{0}}(M \Phi \downarrow)$ : In such a case, we have that $\delta_{\alpha}\left(v_{i}\right)=\delta_{\beta}\left(v_{i}\right)$. Hence, we easily conclude.

Case $v_{i}=\mathrm{f}(n)$ for some $\mathrm{f} \in\{\mathrm{pk}, \mathrm{vk}\}$ and $n \in \mathcal{N}$ : By hypothesis, we know that either new $\mathcal{E} . \Phi \nvdash k$, for all $k \in K_{S}$; or new $\mathcal{E} . \delta(\Phi \downarrow) \nvdash k$, for all $k \in \delta_{\alpha}\left(K_{S}\right) \cup \delta_{\beta}\left(K_{S}\right)$. Since we have shown that $v_{i}$ is deducible from new $\mathcal{E} . \Phi$ and $\delta_{\gamma_{0}}\left(v_{i}\right)$ is deducible from new $\mathcal{E} . \delta(\Phi \downarrow)$, both hypotheses imply that $n \notin \operatorname{dom}\left(\rho_{\alpha}^{+}\right) \cup \operatorname{dom}\left(\rho_{\beta}^{+}\right)$, and so $\delta_{\alpha}\left(v_{i}\right)=\delta_{\beta}\left(v_{i}\right)$.

Case $v_{i} \in \operatorname{dom}\left(\rho_{\alpha}^{+}\right) \cup \operatorname{dom}\left(\rho_{\beta}^{+}\right)$: By hypothesis, we know that either new $\mathcal{E}$. $\Phi \nvdash k$, for all $k \in K_{S}$; or new $\mathcal{E} . \delta(\Phi \downarrow) \forall k$, for all $k \in \delta_{\alpha}\left(K_{S}\right) \cup \delta_{\beta}\left(K_{S}\right)$. Since we have shown that $v_{i}$ is deducible from new $\mathcal{E} . \Phi$ and $\delta_{\gamma_{0}}\left(v_{i}\right)$ is deducible from new $\mathcal{E} . \delta(\Phi \downarrow)$, both hypotheses imply that $v_{i} \notin \operatorname{dom}\left(\rho_{\alpha}^{+}\right) \cup$ $\operatorname{dom}\left(\rho_{\beta}^{+}\right)$and lead us to a contradiction.

Corollary 54: Let $A=(\mathcal{E} ; \mathcal{P} ; \Phi ; \sigma)$ be a derived welltagged extended process and let $\left(\rho_{\alpha}, \rho_{\beta}\right)$ be compatible with $A$, such that $\mathcal{E}=\mathcal{E}_{0} \uplus \mathcal{E}_{\alpha} \uplus \mathcal{E}_{\beta}$, and $\operatorname{fn}(\Phi) \cap\left(\mathcal{E}_{\alpha} \uplus \mathcal{E}_{\beta}\right)=\emptyset$. The two following conditions are equivalent:

1) new $\mathcal{E} . \Phi \nvdash k$ for any $k \in K_{S}$; or

2) new $\mathcal{E} \cdot \delta(\Phi \downarrow) \forall k$ for any $k \in \delta_{\alpha}\left(K_{S}\right) \cup \delta_{\beta}\left(K_{S}\right)$.

with $K_{S}=\left\{t, \operatorname{pk}(t), \operatorname{vk}(t) \mid t \in \operatorname{dom}\left(\rho_{\alpha}^{+}\right) \cup\right.$ $\operatorname{dom}\left(\rho_{\beta}^{+}\right), t$ ground $\}$.

Proof: We prove the two implications separately.

$(2) \Rightarrow(1)$ : Let $k \in K_{S}$ such that new $\mathcal{E}$. $\Phi \vdash k$. In such a case, there exists $M$ such that $f v(M) \subseteq \operatorname{dom}(\Phi)$, $f n(M) \cap \mathcal{E}=\emptyset$, and $M \Phi \downarrow=k \downarrow$. We assume w.l.o.g. that $k \in\left\{t, \operatorname{pk}(t), \operatorname{vk}(t) \mid t \in \operatorname{dom}\left(\rho_{\alpha}^{+}\right)\right.$and $t$ ground $\}$. Let $\gamma \in\{\alpha, \beta\}$. By Lemma 35, we have that $\delta_{\gamma}(M \Phi \downarrow)=\delta_{\gamma}(k \downarrow)$. Thanks to Lemma 53, we have that $\delta_{\gamma}(M \Phi \downarrow)=M \delta(\Phi \downarrow) \downarrow$, and by Definition of $\delta_{\gamma}$, we have that $\delta_{\gamma}(k \downarrow) \in \delta_{\gamma}\left(K_{S}\right)$. Thus, we deduce that there exists $k^{\prime} \in \delta_{\gamma}\left(K_{S}\right)$ such that new $\mathcal{E} . \delta(\Phi \downarrow) \vdash k^{\prime}$.

$(1) \Rightarrow(2):$ Let $k \in \delta_{\gamma}\left(K_{S}\right)$ with $\gamma \in\{\alpha, \beta\}$, and $M$ be a term such that $f v(M) \subseteq \operatorname{dom}(\Phi), f n(M) \cap \mathcal{E}=\emptyset$, and $M \delta(\Phi \downarrow) \downarrow=$ $k \downarrow$. $k \in \delta_{\gamma}\left(K_{S}\right)$ implies the existence of $k^{\prime} \in K_{S}$ such that $k=\delta_{\gamma}\left(k^{\prime}\right)$, and thus such that $M \delta(\Phi \downarrow) \downarrow=\delta_{\gamma}\left(k^{\prime}\right) \downarrow$. Thanks to Lemma 53, we have that $\delta_{\gamma}(M \Phi \downarrow) \downarrow=\delta_{\gamma}\left(k^{\prime}\right) \downarrow$. Now, if $k^{\prime} \in K_{S}$ there must exist $k^{\prime \prime} \in \operatorname{dom}\left(\rho_{\gamma^{\prime}}\right)$ such that either $k^{\prime}=k^{\prime \prime}$, or $k^{\prime}=\mathrm{pk}\left(k^{\prime \prime}\right)$, or $k^{\prime}=\mathrm{vk}\left(k^{\prime \prime}\right)$. In any case, because $\rho_{\gamma^{\prime}}$ is in normal form, we know that $k^{\prime \prime} \downarrow=k^{\prime \prime}$ and thus that $k^{\prime} \downarrow=k^{\prime}$. Hence $M \delta(\Phi \downarrow) \downarrow=\delta_{\gamma}\left(k^{\prime} \downarrow\right) \downarrow$. But, then according to Lemma 37, $\delta_{\gamma}(M \Phi \downarrow)=\delta_{\gamma}(M \Phi \downarrow) \downarrow=\delta_{\gamma}\left(k^{\prime} \downarrow\right) \downarrow=\delta_{\gamma}\left(k^{\prime} \downarrow\right)$. 
Finally, thanks to Lemma 35 we can derive that $M \Phi \downarrow=k^{\prime} \downarrow=$ $k^{\prime}$. This implies that $M \Phi \downarrow \in K_{S}$, and thus there is a term in $K_{S}$ that is deducible from new $\mathcal{E} . \Phi$.

Corollary 55: Let $A=(\mathcal{E} ; \mathcal{P} ; \Phi ; \sigma)$ be a derived extended process and let $\left(\rho_{\alpha}, \rho_{\beta}\right)$ be compatible with $A$ such that $\mathcal{E}=$ $\mathcal{E}_{0} \uplus \mathcal{E}_{\alpha} \uplus \mathcal{E}_{\beta}, f n(\Phi) \cap\left(\mathcal{E}_{\alpha} \uplus \mathcal{E}_{\beta}\right)=\emptyset$, and new $\mathcal{E} . \Phi \not k$ for any $k \in K_{S}$. We have that new $\mathcal{E} . \Phi \sim$ new $\mathcal{E} . \delta(\Phi \downarrow)$.

Proof: The proof directly follows from Lemmas 35 and 53. Indeed, $M \Phi \downarrow=N \Phi \downarrow$ is equivalent to $\delta_{\gamma}(M \Phi \downarrow)=$ $\delta_{\gamma}(N \Phi \downarrow)$ (thanks to Lemma 35), which is equivalent to $M \delta(\Phi \downarrow) \downarrow=N \delta(\Phi \downarrow) \downarrow$ (thanks to Lemma 53).

\section{F. Proof of Theorem 31}

The goal of this section is to prove Theorem 31 . We first state and prove two propositions.

Let $S=\left(\mathcal{E}_{S} ; \mathcal{P}_{S} ; \Phi_{S} ; \sigma_{S}\right)$ and $D=\left(\mathcal{E}_{D} ; \mathcal{P}_{D} ; \Phi_{D} ; \sigma_{D}\right)$. We say that $D=\delta(S)$ if $\mathcal{E}_{S}=\mathcal{E}_{D}, \mathcal{P}_{D}=\delta\left(\mathcal{P}_{S}\right), \Phi_{D \downarrow}=\delta\left(\Phi_{S \downarrow}\right)$, and $\sigma_{D \downarrow}=\delta\left(\sigma_{S \downarrow}\right)$.

Proposition 56: Let $P_{0}$ be a plain coloured process without replication and such that $b n\left(P_{0}\right)=f v\left(P_{0}\right)=\emptyset$. Let $B_{0}$ be an extended coloured biprocess such that:

- $S_{0}=\left(\mathcal{E}_{\alpha} \uplus \mathcal{E}_{\beta} \uplus \mathcal{E}_{0} ; \llbracket P_{0} \rrbracket ; \emptyset ; \emptyset\right) \stackrel{\text { def }}{=}$ fst $\left(B_{0}\right)$,

- $D_{0}=\left(\mathcal{E}_{\alpha} \uplus \mathcal{E}_{\beta} \uplus \mathcal{E}_{0} ; P_{0}^{\prime} ; \emptyset ; \emptyset\right) \stackrel{\text { def }}{=} \operatorname{snd}\left(B_{0}\right)$, and

- $D_{0}=\delta^{\rho_{\alpha}^{+}, \rho_{\beta}^{+}}\left(S_{0}\right)$ for some $\left(\rho_{\alpha}, \rho_{\beta}\right)$, and

- $D_{0}$ does not reveal the value of its assignments w.r.t. $\left(\rho_{\alpha}, \rho_{\beta}\right)$.

For any extended process $S=\left(\mathcal{E}_{S} ; \mathcal{P}_{S} ; \Phi_{S} ; \sigma_{S}\right)$ such that $S_{0} \stackrel{\text { tr }}{\Rightarrow} S$ with $\left(\rho_{\alpha}, \rho_{\beta}\right)$ compatible with $S$, there exists a biprocess $B$ and an extended process $D=\left(\mathcal{E}_{D} ; \mathcal{P}_{D} ; \Phi_{D} ; \sigma_{D}\right)$ such that $B_{0} \stackrel{\text { tr }}{\Rightarrow}$ bi $B$, fst $(B)=S$, snd $(B)=D, D=\delta(S)$ and with $\left(\rho_{\alpha}, \rho_{\beta}\right)$ compatible with $D$.

Proof: Let $\mathcal{E}=\mathcal{E}_{0} \uplus \mathcal{E}_{\alpha} \uplus \mathcal{E}_{\beta}$. We show the result by induction on the length of the derivation. The base case when $S=S_{0}$ is trivial. We simply conclude by considering $B=B_{0}$, and $D=D_{0}$. Now, we assume that $S_{0} \stackrel{\operatorname{tr}^{\prime}}{\Rightarrow} S^{\prime}$ such that $\left(\rho_{\alpha}, \rho_{\beta}\right)$ is compatible with $S^{\prime}$. This means that there exists $S^{\prime}$, tr and $\ell$ such that:

$$
S_{0} \stackrel{\text { tr }}{\Rightarrow} S \stackrel{\ell}{\rightarrow} S^{\prime} \text { with } \mathrm{tr}^{\prime}=\mathrm{tr} \cdot \ell
$$

Moreover, we have that $\left(\rho_{\alpha}, \rho_{\beta}\right)$ is compatible with $S$.

By induction hypothesis, we have that there exists an extended biprocess $B$ and an extended process $D$ such that fst $(B)=S$, snd $(B)=D, B_{0} \stackrel{\text { tr }}{\Rightarrow}{ }_{\mathrm{bi}} B$, and $D=\delta(S)$. We will show by case analysis on the rule involved in $S \stackrel{\ell}{\rightarrow} S^{\prime}$ that exists a biprocess $B$ and an extended process $D^{\prime}=\left(\mathcal{E}_{D}^{\prime} ; \mathcal{P}_{D}^{\prime} ; \Phi_{D}^{\prime} ; \sigma_{D}^{\prime}\right)$ such that $B_{0} \stackrel{\text { tr }}{\Rightarrow}$ bi $B^{\prime}$, fst $\left(B^{\prime}\right)=S^{\prime}$, $\operatorname{snd}\left(B^{\prime}\right)=D^{\prime}, D^{\prime}=\delta\left(S^{\prime}\right)$. Then it will remain to prove that $\left(\rho_{\alpha}, \rho_{\beta}\right)$ compatible with $D^{\prime}$. To do so, we rely on the fact that $\delta\left(\sigma_{S^{\prime}} \downarrow\right)=\sigma_{D^{\prime}} \downarrow$. In particular, by Lemma 35, we have that for all assignment variables $z, z^{\prime}, z \sigma_{S^{\prime}} \downarrow=z^{\prime} \sigma_{S^{\prime}} \downarrow$ is equivalent to $\delta\left(z \sigma_{S^{\prime} \downarrow}\right)=\delta\left(z^{\prime} \sigma_{S^{\prime}} \downarrow\right)$ which is also equivalent to $z \delta\left(\sigma_{S^{\prime}} \downarrow\right)=z^{\prime} \delta\left(\sigma_{S^{\prime}} \downarrow\right)$. Moreover, since $\left(\rho_{\alpha}, \rho_{\beta}\right)$ is compatible with $S^{\prime}$, then for all assignment variable $z \in \operatorname{dom}\left(\rho_{\gamma}\right)$, either $\operatorname{tagroot}\left(z \sigma_{S^{\prime}} \downarrow\right)=\perp$ or tagroot $\left(z \sigma_{S^{\prime}} \downarrow\right) \notin \gamma \cup\{0\}$. Thus, by Lemma 37, we deduce that either $\operatorname{tagroot}\left(\delta_{\gamma}^{\prime}\left(z \sigma_{S^{\prime}} \downarrow\right)\right)=\perp$ or tagroot $\left(\delta_{\gamma}^{\prime}\left(z \sigma_{S^{\prime}} \downarrow\right)\right) \notin \gamma \cup\{0\}$. This allows us to conclude that either $\operatorname{tagroot}\left(z \sigma_{D^{\prime}}\right)=\perp$ or $\operatorname{tagroot}\left(z \sigma_{D^{\prime}} \downarrow\right) \notin \gamma \cup\{0\}$, and so that $\left(\rho_{\alpha}, \rho_{\beta}\right)$ is compatible with $D^{\prime}$.

Let's now prove the core part of the result. Let $S=$ $\left(\mathcal{E}_{S} ; \mathcal{P}_{S} ; \Phi_{S} ; \sigma_{S}\right)$ and $S^{\prime}=\left(\mathcal{E}_{S}^{\prime} ; \mathcal{P}_{S}^{\prime} ; \Phi_{S} ; \sigma_{S}^{\prime}\right)$.

Case of the rule OUT-T. In such a case, we have that $\mathcal{E}_{S}^{\prime}=$ $\mathcal{E}_{S}=\mathcal{E}, \sigma_{S}^{\prime}=\sigma_{S}, \mathcal{P}_{S}=\left\{\operatorname{out}\left(c,[u]_{i}\right)^{i} . Q\right\} \uplus \mathcal{Q}_{S}, \mathcal{P}_{S}^{\prime}=$ $\{Q\} \uplus \mathcal{Q}_{S}$, and $\Phi_{S}^{\prime}=\Phi_{S} \cup\left\{w_{n} \triangleright[u]_{i} \sigma_{S}\right\}$. Furthermore, we have that $\ell=$ new $w_{n}$.out $\left(c, w_{n}\right), c \notin \mathcal{E}$, and $n=\left|\Phi_{S}\right|+1$. Lastly, since $S$ is issued from $\left(\mathcal{E} ; \llbracket P_{0} \rrbracket ; \emptyset ; \emptyset\right)$, we have that $\sigma_{S} \vDash \operatorname{test}_{i}\left([u]_{i}\right)$.

By hypothesis, we have that $D=\delta(S)$. Hence, we have that:

$$
D=\left(\mathcal{E} ;\left\{\operatorname{out}\left(c, \delta_{\gamma}\left([u]_{i}\right)\right) . \delta(Q)\right\} \uplus \delta\left(\mathcal{Q}_{S}\right) ; \Phi_{D} ; \sigma_{D}\right)
$$

with $\Phi_{D \downarrow}=\delta\left(\Phi_{S} \downarrow\right), \sigma_{D \downarrow}=\delta\left(\sigma_{S} \downarrow\right)$, and $\gamma \in\{\alpha, \beta\}$ such that $i \in \gamma$.

Hence, we have that $D \stackrel{\text { new } w_{n} \text {.out }\left(c, w_{n}\right)}{\longrightarrow} D^{\prime}$ where

$$
D^{\prime}=\left(\mathcal{E} ; \delta(Q) \uplus \delta\left(\mathcal{Q}_{S}\right) ; \Phi_{D} \cup\left\{w_{n} \triangleright \delta_{\gamma}\left([u]_{i}\right) \sigma_{D}\right\} ; \sigma_{D}\right) .
$$

Hence, we have that $B \stackrel{\text { new } w_{n} \text {.out }\left(c, w_{n}\right)}{\longrightarrow}$ bi $B^{\prime}$ with $\operatorname{fst}\left(B^{\prime}\right)=$ $S^{\prime}$ and $\operatorname{snd}\left(B^{\prime}\right)=D^{\prime}$. It remains to show that $D^{\prime}=\delta\left(S^{\prime}\right)$, i.e.

$$
\left(\delta_{\gamma}\left([u]_{i}\right) \sigma_{D}\right) \downarrow=\delta_{\gamma}\left([u]_{i} \sigma_{S} \downarrow\right) .
$$

Since $\sigma_{D \downarrow}=\delta\left(\sigma_{S \downarrow}\right)$, we have that:

$$
\left(\delta_{\gamma}\left([u]_{i}\right) \sigma_{D}\right) \downarrow=\left(\delta_{\gamma}\left([u]_{i}\right) \delta\left(\sigma_{S} \downarrow\right)\right) \downarrow
$$

Let $\gamma^{\prime}$ be equal to $\alpha$ if $\gamma=\beta$, and equal to $\beta$ if $\gamma=\alpha$. Each variable that occurs in $[u]_{i}$ also occurs in $\operatorname{dom}\left(\sigma_{S}\right)$ and such a variable is either colored with a color in $\gamma$, or an assignation variable $z_{j}^{\gamma^{\prime}}$. Thus, we have that $\delta_{\gamma}\left([u]_{i}\right)$ only contains variables that are colored with a color in $\gamma$. Hence, we have that

$$
\left(\delta_{\gamma}\left([u]_{i}\right) \delta\left(\sigma_{S \downarrow}\right)\right) \downarrow=\left(\delta_{\gamma}\left([u]_{i}\right) \delta_{\gamma}\left(\sigma_{S} \downarrow\right)\right) \downarrow
$$

Relying on Lemma 38 (note that $\sigma_{S \downarrow} \vDash$ test $_{i}\left([u]_{i}\right)$ ), we have that:

$$
\begin{aligned}
\left(\delta_{\gamma}\left([u]_{i}\right) \sigma_{D}\right) \downarrow & =\left(\delta_{\gamma}\left([u]_{i}\right) \delta_{\gamma}\left(\sigma_{S} \downarrow\right)\right) \downarrow \\
& =\delta_{\gamma}\left([u]_{i}\left(\sigma_{S} \downarrow\right)\right) \downarrow \\
& =\delta_{\gamma}\left([u]_{i}\left(\sigma_{S} \downarrow\right) \downarrow\right) \\
& =\delta_{\gamma}\left([u]_{i} \sigma_{S \downarrow}\right)
\end{aligned}
$$

Case of the rule IN. In such a case, we have that $\mathcal{E}_{S}^{\prime}=\mathcal{E}_{S}$, $\Phi_{S}^{\prime}=\Phi_{S}, \mathcal{P}_{S}=\left\{\operatorname{in}(c, x)^{i} \cdot Q\right\} \uplus \mathcal{Q}_{S}, \mathcal{P}_{S}^{\prime}=\{Q\} \uplus \mathcal{Q}_{S}$, $\sigma_{S}^{\prime}=\sigma_{S} \cup\left\{x \mapsto M \Phi_{S}\right\}$, and $\ell=\operatorname{in}(c, M)$ with $c \notin \mathcal{E}_{S}$, $f v(M) \subseteq \operatorname{dom}\left(\Phi_{S}\right)$ and $f n(M) \cap \mathcal{E}_{S}=\emptyset$.

By hypothesis, we have that $D=\delta(S)$. Hence, we have that:

$$
D=\left(\mathcal{E} ;\{\operatorname{in}(c, x) . \delta(Q)\} \uplus \delta\left(\mathcal{Q}_{S}\right) ; \Phi_{D} ; \sigma_{D}\right)
$$

with $\Phi_{D \downarrow}=\delta\left(\Phi_{S \downarrow}\right), \sigma_{D \downarrow}=\delta\left(\sigma_{S \downarrow}\right)$. Let $\gamma \in\{\alpha, \beta\}$ such that $i \in \gamma$. 
Hence, we have that $D \stackrel{\text { in }(c, M)}{\longrightarrow} D^{\prime}$ where

$$
D^{\prime}=\left(\mathcal{E} ; \delta(Q) \uplus \delta\left(\mathcal{Q}_{S}\right) ; \Phi_{D} ; \sigma_{D} \cup\left\{x \mapsto M \Phi_{D}\right\}\right) .
$$

Hence, we have that $B \stackrel{\text { in }(c, M)}{\longrightarrow}$ bi $B^{\prime}$ with fst $\left(B^{\prime}\right)=S^{\prime}$ and snd $\left(B^{\prime}\right)=D^{\prime}$. It remains to show that $D^{\prime}=\delta\left(S^{\prime}\right)$, i.e.

$$
\left(M \Phi_{D}\right) \downarrow=\delta_{\gamma}\left(M \Phi_{S \downarrow}\right) .
$$

By hypothesis, we know that $D_{0}$ does not reveal the values of its assignment variables w.r.t. $\left(\rho_{\alpha}, \rho_{\beta}\right)$. Hence, for all assignment variable $x$ of color $\alpha$ (resp. $\beta$ ) in $\operatorname{dom}\left(\sigma_{D}\right)$, for all $k \in\left\{k, \operatorname{pk}(k), \operatorname{vk}(k) \mid k=x \sigma_{D} \vee k=x \rho_{\alpha}\left(\right.\right.$ resp. $\left.\left.x \rho_{\beta}\right)\right\}$, $k$ is not deducible in new $\mathcal{E} . \Phi_{D}$. We denote $K$ this set.

Let $K_{S}=\left\{t, \mathrm{pk}(t), \mathrm{vk}(t) \mid t \in \operatorname{dom}\left(\rho_{\alpha}^{+}\right) \cup\right.$ $\operatorname{dom}\left(\rho_{\beta}^{+}\right), t$ ground $\}$ We know that $\sigma_{D \downarrow}=\delta\left(\sigma_{S \downarrow}\right)$, and by definition of $\rho_{\alpha}^{+}$and $\rho_{\beta}^{+}$, we have that $K=\delta_{\alpha}\left(K_{S}\right) \cup \delta_{\beta}\left(K_{S}\right)$. We have also that $\Phi_{D \downarrow}=\delta\left(\Phi_{S \downarrow} \downarrow\right)$. Hence, we deduce that new $\mathcal{E} . \delta\left(\Phi_{S} \downarrow\right) \forall k$ for any $k \in \delta_{\alpha}\left(K_{S}\right) \cup \delta_{\beta}\left(K_{S}\right)$ This allow us to apply Lemma 53 and thus to obtain that:

$$
\left(M \Phi_{D}\right) \downarrow=\left(M\left(\Phi_{D} \downarrow\right)\right) \downarrow=\left(M \delta\left(\Phi_{S} \downarrow\right)\right) \downarrow=\delta_{\gamma}\left(M \Phi_{S \downarrow}\right) .
$$

Case of the rule THEN. In such a case, we have that $\mathcal{E}_{S}^{\prime}=\mathcal{E}_{S}$, $\Phi_{S}^{\prime}=\Phi_{S}, \sigma_{S}^{\prime}=\sigma_{S}, \mathcal{P}_{S}=\left\{P_{S}\right\} \uplus \mathcal{Q}_{S}$, and $\mathcal{P}_{S}^{\prime}=\left\{P_{S}^{\prime}\right\} \uplus \mathcal{Q}_{S}$ where $P_{S}$ and $P_{S}^{\prime}$ are as follows:

- Case a: a test before an output.

$$
\begin{aligned}
& P_{S}=\text { if } \operatorname{test}_{i}\left([v]_{i}\right) \text { then out }\left(c,[v]_{i}\right)^{i} \cdot Q_{S} \\
& P_{S}^{\prime}=\operatorname{out}\left(u,[v]_{i}\right)^{i} \cdot Q_{S} \\
& \sigma_{S} \vDash \operatorname{test}_{i}\left([v]_{i}\right)
\end{aligned}
$$

for some $i \in\{1, \ldots, p\}$.

- Case b: a test before an assignation.

$$
\begin{aligned}
& P_{S}=\text { if } \operatorname{test}_{i}\left([v]_{i}\right) \text { then }\left[z:=[v]_{i}\right]^{i} \cdot Q_{S} \\
& P_{S}^{\prime}=\left\{\left[z:=[v]_{i}\right]^{i} \cdot Q_{S}\right. \\
& \sigma_{S} \vDash \operatorname{test}_{i}\left([v]_{i}\right)
\end{aligned}
$$

for some $i \in\{1, \ldots, p\}$.

- Case c: a test before a conditional.

$$
\begin{aligned}
& P_{S}=\text { if test }{ }_{i}\left([\varphi]_{i}\right) \text { then }\left(\text { if }[\varphi]_{i} \text { then } Q_{S}^{1} \text { else } Q_{S}^{2}\right) \\
& P_{S}^{\prime}=\text { if }[\varphi]_{i} \text { then } Q_{S}^{1} \text { else } Q_{S}^{2} \\
& \sigma_{S} \vDash \operatorname{test~}_{i}\left([\varphi]_{i}\right)
\end{aligned}
$$

for some $i \in\{1, \ldots, p\}$.

- Case d: a test of a conditional.

$$
\begin{aligned}
& P_{S}=\operatorname{if}[\varphi]_{i} \text { then } Q_{S}^{1} \text { else } Q_{S}^{2} \\
& P_{S}^{\prime}=Q_{S}^{1} \\
& \sigma_{S} \vDash[\varphi]_{i} \text { and } \sigma_{S} \vDash \operatorname{test}_{i}\left([\varphi]_{i}\right)
\end{aligned}
$$

for some $i \in\{1, \ldots, p\}$.

Each case can be handled in a similar way. Note that we rely on Corollary 39 instead of Lemma 40 to establish the result in Case $d$. We assume that we are in the first case. Let $\gamma \in\{\alpha, \beta\}$ such that $i \in \gamma$. By hypothesis, we have that $D=\delta(S)$. Hence, we have that $D$ is equal to

$$
\begin{aligned}
& \left(\mathcal{E} ;\left\{\text { if } \operatorname{test}_{i}\left(\delta_{\gamma}\left([v]_{i}\right)\right)\right. \text { then }\right. \\
& \left.\left.\quad \operatorname{out}\left(c, \delta_{\gamma}\left([v]_{i}\right)\right) . \delta\left(Q_{S}\right)\right\} \uplus \mathcal{Q}_{S} ; \Phi_{D} ; \sigma_{D}\right)
\end{aligned}
$$

with $\Phi_{D \downarrow}=\delta\left(\Phi_{S \downarrow}\right)$, and $\sigma_{D \downarrow}=\delta\left(\sigma_{S \downarrow}\right)$.

Since $\sigma_{S} \vDash \operatorname{test}_{i}\left([v]_{i}\right)$, we have also that $\left(\sigma_{S} \downarrow\right) \vDash$ test $_{i}\left([v]_{i}\right)$. Thanks to Lemma 40, we deduce that $\delta_{\gamma}\left(\sigma_{S} \downarrow\right) \vDash$ test $_{i}\left(\delta_{\gamma}\left([v]_{i}\right)\right)$. Actually, each variable that occurs in $\operatorname{test}_{i}\left(\delta_{\gamma}\left([v]_{i}\right)\right)$ is a variable that occurs in $\operatorname{dom}\left(\sigma_{S}\right)$ and such a variable is necessarily colored with a color in $\gamma$. Hence, we have also that:

$$
\delta\left(\sigma_{S \downarrow} \downarrow\right) \operatorname{test}_{i}\left(\delta_{\gamma}\left([v]_{i}\right)\right) .
$$

Hence, we have that $D \stackrel{\tau}{\rightarrow} D^{\prime}$ where

$$
D^{\prime}=\left(\mathcal{E} ;\left\{\operatorname{out}\left(u, \delta_{\gamma}\left([v]_{i}\right)\right) . \delta\left(Q_{S}\right)\right\} \uplus \delta\left(\mathcal{Q}_{S}\right) ; \Phi_{D} ; \sigma_{D}\right) .
$$

Hence, we have that $B \stackrel{\tau}{\rightarrow}$ bi $B^{\prime}$ with fst $\left(B^{\prime}\right)=S^{\prime}$ and $\operatorname{snd}\left(B^{\prime}\right)=D^{\prime}$. We also have that $D^{\prime}=\delta\left(S^{\prime}\right)$.

Case of the rule ELSE. This case is similar to the previous one.

Case of the rule ASSGN. In such a case, we have that $\mathcal{E}_{S}^{\prime}=\mathcal{E}_{S}$, $\Phi_{S}^{\prime}=\Phi_{S}, \mathcal{P}_{S}=\left\{\left[x:=[v]_{i}\right] . Q\right\} \uplus \mathcal{Q}_{S}, \mathcal{P}_{S}^{\prime}=\{Q\} \uplus \mathcal{Q}_{S}$, $\sigma_{S}^{\prime}=\sigma_{S} \cup\left\{x \mapsto[v]_{i} \sigma_{S}\right\}$, and $\ell=\tau$. Lastly, since $S$ is issued from $\left(\mathcal{E} ; \llbracket P_{0} \rrbracket ; \emptyset ; \emptyset\right)$, we have that $\sigma_{S} \vDash$ test $_{i}\left([v]_{i}\right)$.

By hypothesis, we have that $D=\delta(S)$. Hence, we have that:

$$
D=\left(\mathcal{E} ;\left[x:=\delta\left([v]_{i}\right)\right] . \delta(Q) \uplus \delta\left(\mathcal{Q}_{S}\right) ; \Phi_{D} ; \sigma_{D}\right)
$$

with $\Phi_{D \downarrow}=\delta\left(\Phi_{S \downarrow}\right), \sigma_{D \downarrow}=\delta\left(\sigma_{S \downarrow}\right)$, and $\gamma \in\{\alpha, \beta\}$ such that $i \in \gamma$.

Hence, we have that $D \stackrel{\tau}{\rightarrow} D^{\prime}$ where

$$
D^{\prime}=\left(\mathcal{E} ; \delta(Q) \uplus \delta\left(\mathcal{Q}_{S}\right) ; \Phi_{D} ; \sigma_{D} \cup\left\{x \mapsto \delta_{\gamma}\left([v]_{i}\right) \sigma_{D}\right\}\right) .
$$

Hence, we have that $B \stackrel{\tau}{\rightarrow} B^{\prime}$ with fst $\left(B^{\prime}\right)=S^{\prime}$ and $\operatorname{snd}\left(B^{\prime}\right)=D^{\prime}$. It remains to show that $D^{\prime}=\delta\left(S^{\prime}\right)$, i.e.

$$
\left(\delta_{\gamma}\left([v]_{i}\right) \sigma_{D}\right) \downarrow=\delta_{\gamma}\left([v]_{i} \sigma_{S \downarrow}\right) .
$$

This can be done as in the case of the rule OUT-T.

Case of the rule COMM. In such a case, we have that $\mathcal{E}_{S}^{\prime}=$ $\mathcal{E}_{S}, \Phi_{S}^{\prime}=\Phi_{S}, \mathcal{P}_{S}=\left\{\operatorname{out}\left(c,[u]_{i}\right)^{i} . Q_{1} ;\right.$ in $\left.(c, x)^{i^{\prime}} \cdot Q_{2}\right\} \uplus \mathcal{Q}_{S}$, $\sigma_{S}^{\prime}=\sigma_{S} \cup\left\{x \mapsto[u]_{i} \sigma_{S}\right\}$, and $\ell=\tau$. Lastly, since $S$ is issued from $\left(\mathcal{E} ; \llbracket P_{0} \rrbracket ; \emptyset ; \emptyset\right)$, we have that $\sigma_{S} \vDash \operatorname{test}_{i}\left([u]_{i}\right)$.

By hypothesis, we have that $D=\delta(S)$. Hence, we have that $D$ is equal to

$\left(\mathcal{E} ;\left\{\operatorname{out}\left(c, \delta_{\gamma}\left([u]_{i}\right)\right) . \delta\left(Q_{1}\right) ; \operatorname{in}(c, x) . \delta\left(Q_{2}\right)\right\} \uplus \delta\left(\mathcal{Q}_{S}\right) ; \Phi_{D} ; \sigma_{D}\right)$

with $\Phi_{D \downarrow}=\delta\left(\Phi_{S \downarrow} \downarrow\right), \sigma_{D \downarrow}=\delta\left(\sigma_{S \downarrow}\right)$.

Let $\gamma, \gamma^{\prime} \in\{\alpha, \beta\}$ such that $i \in \gamma$, and $i^{\prime} \in \gamma^{\prime}$. Hence, we have that $D \stackrel{\tau}{\rightarrow} D^{\prime}$ where $D^{\prime}$ is equal to:

$$
\left(\mathcal{E} ;\left\{\delta\left(Q_{1}\right) ; \delta\left(Q_{2}\right)\right\} \uplus \delta\left(\mathcal{Q}_{S}\right) ; \Phi_{D} ; \sigma_{D} \cup\left\{\left(x \mapsto \delta_{\gamma}\left([u]_{i}\right) \sigma_{D}\right)^{i^{\prime}}\right\}\right) .
$$

Hence, we have that $B \stackrel{\tau}{\rightarrow} B^{\prime}$ for some biprocess $B^{\prime}$ such that $\operatorname{fst}\left(B^{\prime}\right)=S^{\prime}$ and $\operatorname{snd}\left(B^{\prime}\right)=D^{\prime}$. It remains to show that $D^{\prime}=\delta\left(S^{\prime}\right)$, i.e.

$$
\left(\delta_{\gamma}\left([u]_{i}\right) \sigma_{D}\right) \downarrow=\delta_{\gamma^{\prime}}\left(\left([u]_{i} \sigma_{S}\right) \downarrow\right)
$$

If $\gamma=\gamma^{\prime}$, then this can be done as in the previous cases. 
Otherwise, since names can only be shared through assignments, and assignments only concern variables/terms of base type, we necessarily have that $c \notin \mathcal{E}$. Hence, we have that $S \stackrel{\nu w_{n} . \text { out }\left(c, w_{n}\right)}{\longrightarrow} S_{\text {out }}$ where:

$S_{\text {out }}=\left(\mathcal{E} ;\left\{Q_{1} ;\right.\right.$ in $\left.\left.(c, x) \cdot Q_{2}\right\} \uplus \mathcal{Q}_{S} ; \Phi_{S} \cup\left\{w_{n} \triangleright[u]_{i} \sigma_{S}\right\} ; \sigma_{S}\right)$

Note that $\left(\rho_{\alpha}, \rho_{\beta}\right)$ is still compatible with $S_{\text {out }}$. We would like to apply Lemma 53 with $M=w_{n}$ on the frame of $S_{\text {out }}$ which requires an hypothesis of non deductibility of the shared key. For these, we will rely on our hypothesis that $D_{0}$ does not reveal the values of its assignments w.r.t. $\left(\rho_{\alpha}, \rho_{\beta}\right)$ :

Let $\Phi_{S}^{\prime}=\Phi_{S} \cup\left\{w_{n} \triangleright[u]_{i} \sigma_{S}\right\}$. We already proved our induction result for the rule OUT-T. Hence, we deduce that there exists $D_{\text {out }}$ such that $D \stackrel{\nu w_{n} \text {.out }\left(c, w_{n}\right)}{\longrightarrow} D_{\text {out }}$ where $D_{\text {out }}=\left(\mathcal{E} ; \mathcal{P}_{\text {out }} ; \Phi_{D}^{\prime} ; \sigma_{D}\right), \Phi_{D}^{\prime}=\Phi_{D} \cup\left\{w_{n} \triangleright \delta_{\gamma}\left([u]_{i}\right) \sigma_{D}\right\}$. Moreover, it implies that $\left.\Phi_{D}^{\prime} \downarrow=\delta\left(\Phi_{S}^{\prime} \downarrow\right\}\right)$ and $\sigma_{D} \downarrow=\delta\left(\sigma_{S} \downarrow\right)$. As mentioned, by hypothesis, we know that $D_{0}$ does not reveal the values of its assignments w.r.t. $\left(\rho_{\alpha}, \rho_{\beta}\right)$. Hence, for all assignment variable $x$ of color $\alpha$ (resp. $\beta$ ) in $\operatorname{dom}\left(\sigma_{D}\right)$, for all $k \in\left\{k, \operatorname{pk}(k), \operatorname{vk}(k) \mid k=x \sigma_{D} \vee k=x \rho_{\alpha}\right.$ (resp. $\left.\left.x \rho_{\beta}\right)\right\}$, $k$ is not deducible in new $\mathcal{E} . \Phi_{D}^{\prime}$. We denote by $K$ such a set. Let $K_{S}=\left\{t, \operatorname{pk}(t), \operatorname{vk}(t) \mid t \in \operatorname{dom}\left(\rho_{\alpha}^{+}\right) \cup\right.$ $\operatorname{dom}\left(\rho_{\beta}^{+}\right), t$ ground $\}$. Since $\sigma_{D} \downarrow=\delta\left(\sigma_{S} \downarrow\right)$, and by definition of $\rho_{\alpha}^{+}$and $\rho_{\beta}^{+}$, we deduce that $K=\delta_{\alpha}\left(K_{S}\right) \cup \delta_{\beta}\left(K_{S}\right)$. Moreover, we have that $\Phi_{D}^{\prime} \downarrow=\delta\left(\Phi_{S}^{\prime} \downarrow\right)$. Hence, we deduce that new $\mathcal{E} . \delta\left(\Phi_{S}^{\prime} \downarrow\right) \forall k$ for any $k \in \delta_{\alpha}\left(K_{S}\right) \cup \delta_{\beta}\left(K_{S}\right)$. This allow us to apply Lemma 53 with $M=w_{n}$ and so we deduce that $\delta_{\gamma}\left([u]_{i} \sigma_{S} \downarrow\right)=\delta_{\gamma^{\prime}}\left([u]_{i} \sigma_{S} \downarrow\right)$. Hence, we can conclude as in the previsous case.

Case of the rule PAR. It is easy to see that the result holds for this case.

Note that the rules NEW and REPL can not be triggered since the processes under study do not contain bounded names and replication.

Proposition 57: Let $P_{0}$ be a plain colored process without replication and such that $b n\left(P_{0}\right)=f v\left(P_{0}\right)=\emptyset$. Let $B_{0}$ be an extended colored biprocess such that:

- $S_{0}=\left(\mathcal{E}_{\alpha} \uplus \mathcal{E}_{\beta} \uplus \mathcal{E}_{0} ; \llbracket P_{0} \rrbracket ; \emptyset ; \emptyset\right) \stackrel{\text { def }}{=} \mathrm{fst}\left(B_{0}\right)$,

- $D_{0}=\left(\mathcal{E}_{\alpha} \uplus \mathcal{E}_{\beta} \uplus \mathcal{E}_{0} ; P_{0}^{\prime} ; \emptyset ; \emptyset\right) \stackrel{\text { def }}{=} \operatorname{snd}\left(B_{0}\right)$, and

- $D_{0}=\delta^{\rho_{\alpha}^{+}, \rho_{\beta}^{+}}\left(S_{0}\right)$ for some $\left(\rho_{\alpha}, \rho_{\beta}\right)$.

- $D_{0}$ does not reveal the value of its assignments w.r.t. $\left(\rho_{\alpha}, \rho_{\beta}\right)$.

For any extended process $D=\left(\mathcal{E}_{D} ; \mathcal{P}_{D} ; \Phi_{D} ; \sigma_{D}\right)$ such that $D_{0} \stackrel{\text { tr }}{\Rightarrow} D$ with $\left(\rho_{\alpha}, \rho_{\beta}\right)$ compatible with $D$, there exists a biprocess $B$ and an extended process $S=\left(\mathcal{E}_{S} ; \mathcal{P}_{S} ; \Phi_{S} ; \sigma_{S}\right)$ such that $B_{0} \stackrel{\text { tr }}{\Rightarrow}{ }_{\mathrm{bi}} B$, , fst $(B)=S$, $\operatorname{snd}(B)=D$, and $D=\delta(S)$.

Proof: We show the result by induction on the length of the derivation. The base case when $D_{0}=D$ is trivial. We simply conclude by considering $B=B_{0}$, and $S=S_{0}$. Now, we assume that $D_{0} \stackrel{\operatorname{tr}^{\prime}}{\Rightarrow} D^{\prime}$ such that $\left(\rho_{\alpha}, \rho_{\beta}\right)$ is compatible with $D^{\prime}$. This means that there exist $D, \operatorname{tr}$, and $\ell$ such that:

$$
D_{0} \stackrel{\text { tr }}{\Rightarrow} D \stackrel{\ell}{\rightarrow} D^{\prime} \text { with } \operatorname{tr}^{\prime}=\operatorname{tr} \cdot \ell
$$

Note that we necessarily have that $\left(\rho_{\alpha}, \rho_{\beta}\right)$ is compatible with $D$.

By induction hypothesis, we have that there exists an extended biprocess $B$ and an extended process $S$ such that fst $(B)=S, \operatorname{snd}(B)=D, B_{0} \stackrel{\text { tr }}{\Rightarrow} \mathrm{bi} B$, and $D=\delta(S)$. We show the result by case analysis on the rule involved in $D \stackrel{\ell}{\rightarrow} D^{\prime}$. Let $D=\left(\mathcal{E}_{D} ; \mathcal{P}_{D} ; \Phi_{D} ; \sigma_{D}\right)$ and $D^{\prime}=\left(\mathcal{E}_{D}^{\prime} ; \mathcal{P}_{D}^{\prime} ; \Phi_{D}^{\prime} ; \sigma_{D}^{\prime}\right)$. First, note that since $D$ is issued from $D_{0}=\delta\left(S_{0}\right)$ and $S_{0}=\left(\mathcal{E} ; \llbracket P_{0} \rrbracket ; \emptyset ; \emptyset\right)$, we know that terms invovled in $D$ are tagged and obtained through the $\delta$ transformation.

Case of the rule Out-T. In such a case, we have that $\mathcal{E}_{D}^{\prime}=\mathcal{E}_{D}, \sigma_{D}^{\prime}=\sigma_{D}, \mathcal{P}_{D}=\left\{\operatorname{out}\left(c, \delta\left([v]_{i}\right)\right) . \delta\left(Q_{S}\right)\right\} \uplus \delta\left(\mathcal{Q}_{S}\right)$, $\mathcal{P}_{D}^{\prime}=\left\{\delta\left(Q_{D}\right)\right\} \uplus \delta\left(\mathcal{Q}_{D}\right)$, and $\Phi_{D}^{\prime}=\Phi_{D} \cup\left\{w_{n} \triangleright \delta_{\gamma}\left([v]_{i}\right) \sigma_{D}\right\}$ with $\gamma \in\{\alpha, \beta\}$ such that $i \in \gamma$. Furthermore, we have that $\ell=$ new $w_{n}$.out $\left(c, w_{n}\right), c \notin \mathcal{E}_{D}$, and $n=\left|\Phi_{D}\right|+1$. We have also

$$
\left.S=\left(\mathcal{E} ;\left\{\operatorname{out}\left(c,[v]_{i}\right) \cdot Q_{S}\right\} \uplus \mathcal{Q}_{S}\right) ; \Phi_{S} ; \sigma_{S}\right)
$$

with $\Phi_{D \downarrow}=\delta\left(\Phi_{S} \downarrow\right)$, and $\sigma_{D \downarrow}=\delta\left(\sigma_{S} \downarrow\right)$. Hence, we have that $S \stackrel{\text { new } w_{n} \text {.out }\left(c, w_{n}\right)}{\longrightarrow} S^{\prime}$ where

$$
S^{\prime}=\left(\mathcal{E} ; Q_{S} \uplus \mathcal{Q}_{S} ; \Phi_{S} \cup\left\{w_{n} \triangleright[v]_{i} \sigma_{S}\right\} ; \sigma_{S}\right) .
$$

Hence, we have that $B \stackrel{\text { new } w_{n} \text {.out }\left(c, w_{n}\right)}{\longrightarrow}{ }_{\mathrm{bi}} B^{\prime}$ with fst $\left(B^{\prime}\right)=S^{\prime}$ and $\operatorname{sid}\left(B^{\prime}\right)=D^{\prime}$. It remains to show that $D^{\prime}=\delta\left(S^{\prime}\right)$, i.e.

$$
\left(\delta_{\gamma}\left([v]_{i}\right) \sigma_{D}\right) \downarrow=\delta_{\gamma}\left([v]_{i} \sigma_{S} \downarrow\right)
$$

Since $D$ is issued from $\left(\mathcal{E} ; \delta\left(\llbracket P_{0} \rrbracket\right) ; \emptyset ; \emptyset\right)$ and $B_{0} \stackrel{\text { tr }}{\Rightarrow}{ }_{\mathrm{bi}} B$, we have that $\sigma_{D} \vDash \operatorname{test}_{i}\left(\delta_{\gamma}\left([v]_{i}\right)\right)$ and $\sigma_{S} \vDash$ test $_{i}\left([v]_{i}\right)$.

Since $\sigma_{D} \downarrow=\delta\left(\sigma_{S} \downarrow\right)$, we have that:

$$
\left(\delta_{\gamma}\left([v]_{i}\right) \sigma_{D}\right) \downarrow=\left(\delta_{\gamma}\left([v]_{i}\right) \delta\left(\sigma_{S} \downarrow\right)\right) \downarrow
$$

Let $\gamma^{\prime}$ be equal to $\alpha$ if $\gamma=\beta$, and equal to $\beta$ if $\gamma=\alpha$. Each variable that occurs in $[v]_{i}$ also occurs in $\operatorname{dom}\left(\sigma_{S}\right)$ and such a variable is either colored with a color in $\gamma$, or an assignation variable $z_{j}^{\gamma^{\prime}}$. Thus, we have that $\delta_{\gamma}\left([v]_{i}\right)$ only contains variables that are colored with a color in $\gamma$. Hence, we have that

$$
\left(\delta_{\gamma}\left([v]_{i}\right) \delta\left(\sigma_{S} \downarrow\right)\right) \downarrow=\left(\delta_{\gamma}\left([v]_{i}\right) \delta_{\gamma}\left(\sigma_{S} \downarrow\right)\right) \downarrow
$$

Relying on Lemma 38 (note that $\sigma_{S} \downarrow \vDash$ test $_{i}\left([v]_{i}\right)$ ), we have that:

$$
\begin{aligned}
\left(\delta_{\gamma}\left([v]_{i}\right) \sigma_{D}\right) \downarrow & =\left(\delta_{\gamma}\left([v]_{i}\right) \delta_{\gamma}\left(\sigma_{S} \downarrow\right)\right) \downarrow \\
& =\delta_{\gamma}\left([v]_{i}\left(\sigma_{S} \downarrow\right)\right) \downarrow \\
& =\delta_{\gamma}\left([v]_{i}\left(\sigma_{S} \downarrow\right) \downarrow\right) \\
& =\delta_{\gamma}\left([v]_{i} \sigma_{S} \downarrow\right)
\end{aligned}
$$

Case of the rule IN. In such a case, we have that $\mathcal{E}_{D}^{\prime}=\mathcal{E}_{D}$, $\Phi_{D}^{\prime}=\Phi_{D}, \mathcal{P}_{D}=\left\{\in(c, x)^{i} . \delta\left(Q_{S}\right\} \uplus \delta\left(\mathcal{Q}_{S}\right), \mathcal{P}_{D}^{\prime}=\right.$ $\left\{\delta\left(Q_{S}\right)\right\} \uplus \delta\left(\mathcal{Q}_{S}\right), \sigma_{D}^{\prime}=\sigma_{D} \cup\left\{x \mapsto M \Phi_{D}\right\}$, and $\ell=$ in $(c, M)$ with $c \notin \mathcal{E}_{D}, f v(M) \subseteq \operatorname{dom}\left(\Phi_{D}\right)$, and $f n(M) \cap$ $\mathcal{E}_{D}=\emptyset$. Moreover, we have that:

$$
S=\left(\mathcal{E} ;\left\{\operatorname{in}(c, x) . Q_{S}\right\} \uplus \mathcal{Q}_{S} ; \Phi_{S} ; \sigma_{S}\right)
$$


with $\Phi_{D \downarrow}=\delta\left(\Phi_{S \downarrow}\right)$, and $\sigma_{D \downarrow}=\delta\left(\sigma_{S \downarrow}\right)$. Let $\gamma \in\{\alpha, \beta\}$ such that $i \in \gamma$.

Hence, we have that $S \stackrel{\text { in }(c, M)}{\longrightarrow} S^{\prime}$ where

$$
S^{\prime}=\left(\mathcal{E} ;\left\{Q_{S}\right\} \uplus \mathcal{Q}_{S} ; \Phi_{S} ; \sigma_{S} \cup\left\{x \mapsto M \Phi_{S}\right) .\right.
$$

Hence, we have that $B \stackrel{\text { in }(c, M)}{\longrightarrow}$ bi $B^{\prime}$ with fst $\left(B^{\prime}\right)=S^{\prime}$, and snd $\left(B^{\prime}\right)=D^{\prime}$. It remains to show that $D^{\prime}=\delta\left(S^{\prime}\right)$, i.e.

$$
\left(M \Phi_{D}\right) \downarrow=\delta_{\gamma}\left(M \Phi_{S \downarrow}\right) .
$$

By hypothesis, we know that $D_{0}$ does not reveal the value of its assignments w.r.t. $\left(\rho_{\alpha}, \rho_{\beta}\right)$. Since $\Phi_{D \downarrow}=\delta\left(\Phi_{S \downarrow} \downarrow\right)$ and $\sigma_{D} \downarrow=\delta\left(\sigma_{S} \downarrow\right)$. Hence, by following the definition of $\rho_{\alpha}^{+}$and $\rho_{\beta}^{+}$, we deduce that the hypothesis of Lemma 53 are satisfied. Hence, by relying on it, we have that:

$$
\left(M \Phi_{D}\right) \downarrow=\left(M\left(\Phi_{D} \downarrow\right)\right) \downarrow=\left(M \delta\left(\Phi_{S \downarrow}\right)\right) \downarrow=\delta_{\gamma}\left(M \Phi_{S \downarrow}\right) .
$$

Case of the rule THEN. In such a case, we have that $\mathcal{E}_{D}^{\prime}=\mathcal{E}_{D}$, $\Phi_{D}^{\prime}=\Phi_{D}, \sigma_{D}^{\prime}=\sigma_{D}, \mathcal{P}_{D}=\left\{P_{D}\right\} \uplus \mathcal{Q}_{D}$, and $\mathcal{P}_{D}^{\prime}=\left\{P_{D}^{\prime}\right\} \uplus$ $\mathcal{Q}_{D}$ where $P_{D}$ and $P_{D}^{\prime}$ are as follows:

- Case a: a test before an output.

$$
\begin{aligned}
& P_{D}=\text { iftest } \operatorname{tes}_{i}\left(\delta_{\gamma}\left([v]_{i}\right)\right) \text { then out }\left(c, \delta_{\gamma}\left([v]_{i}\right)\right)^{i} \cdot \delta\left(Q_{S}\right) \\
& P_{D}^{\prime}=\operatorname{out}\left(u, \delta_{\gamma}\left([v]_{i}\right)\right)^{i} . \delta\left(Q_{S}\right) \\
& \sigma_{D} \vDash \operatorname{test}_{i}\left(\delta_{\gamma}\left([v]_{i}\right)\right)
\end{aligned}
$$

for some $i \in\{1, \ldots, p\}$ and $\gamma \in\{\alpha, \beta\}$ such that $i \in \gamma$.

- Case b: a test before an assignation.

$$
\begin{aligned}
& P_{D}=\text { if } \operatorname{test}_{i}\left(\delta_{\gamma}\left([v]_{i}\right)\right) \text { then }\left[z:=\delta_{\gamma}\left([v]_{i}\right)\right]^{i} . \delta\left(Q_{S}\right) \\
& P_{D}^{\prime}=\left\{\left[z:=\delta_{\gamma}\left([v]_{i}\right)\right]^{i} . \delta\left(Q_{S}\right)\right. \\
& \sigma_{D} \vDash \operatorname{test}_{i}\left(\delta_{\gamma}\left([v]_{i}\right)\right)
\end{aligned}
$$

for some $i \in\{1, \ldots, p\}$ and $\gamma \in\{\alpha, \beta\}$ such that $i \in \gamma$.

- Case c: a test before a conditional.

$$
\begin{aligned}
& P_{D}=\operatorname{iftest}\left(\delta_{\gamma}\left([\varphi]_{i}\right)\right) \text { then } \\
& \left(\operatorname{if}[\varphi]_{i} \text { then } \delta\left(Q_{S}^{1}\right) \text { else } \delta\left(Q_{S}^{2}\right)\right) \\
& P_{D}^{\prime}=\operatorname{if}[\varphi]_{i} \text { then } \delta\left(Q_{S}^{1}\right) \text { else } \delta\left(Q_{S}^{2}\right) \\
& \sigma_{D} \vDash \operatorname{test}_{i}\left(\delta_{\gamma}\left([\varphi]_{)} i\right)\right.
\end{aligned}
$$

for some $i \in\{1, \ldots, p\}$ and $\gamma \in\{\alpha, \beta\}$ such that $i \in \gamma$. - Case d: a test of a conditional.

$$
\begin{aligned}
& P_{D}=\operatorname{if} \delta_{\gamma}\left([\varphi]_{i}\right) \text { then } \delta\left(Q_{S}^{1}\right) \text { else } \delta\left(Q_{S}^{2}\right) \\
& P_{D}^{\prime}=\delta\left(Q_{S}^{1}\right) \\
& \sigma_{D} \vDash \delta_{\gamma}\left([\varphi]_{i}\right) \text { and } \sigma_{D} \vDash \operatorname{test}_{i}\left(\delta_{\gamma}\left([\varphi]_{i}\right)\right)
\end{aligned}
$$

for some $i \in\{1, \ldots, p\}$ and $\gamma \in\{\alpha, \beta\}$ such that $i \in \gamma$. Each case can be handled in a similar way. Note that we rely in addition on Corollary 39 instead of Lemma 40 to establish the result in case $d$. We assume that we are in the first case. Let $\gamma \in\{\alpha, \beta\}$ such that $i \in \gamma$. We have that $S$ is equal to

$$
\left(\mathcal{E} ;\left\{\text { if } \operatorname{test}_{i}\left([v]_{i}\right) \text { then out }\left(c,[v]_{i}\right)^{i} . Q_{S}\right\} \uplus \mathcal{Q}_{S} ; \Phi_{S} ; \sigma_{S}\right)
$$

with $\Phi_{D \downarrow}=\delta\left(\Phi_{S \downarrow}\right)$, and $\sigma_{D \downarrow}=\delta\left(\sigma_{S \downarrow}\right)$.

Since $\sigma_{D} \vDash \operatorname{test}_{i}\left(\delta_{\gamma}\left([v]_{i}\right)\right)$, we have $\left(\sigma_{D \downarrow}\right) \vDash$ $\operatorname{test}_{i}\left(\delta_{\gamma}\left([v]_{i}\right)\right)$, and thus $\delta\left(\sigma_{S} \downarrow\right) \vDash \operatorname{test}_{i}\left(\delta_{\gamma}\left([v]_{i}\right)\right)$. As in the previous cases, we deduce that $\delta_{\gamma}\left(\sigma_{S \downarrow} \downarrow\right) \vDash \operatorname{test}_{i}\left(\delta_{\gamma}\left([v]_{i}\right)\right)$.
Thanks to Lemma 40, we deduce that $\sigma_{S \downarrow} \vDash \operatorname{test}_{i}\left([v]_{i}\right)$. Hence, we have that $S \stackrel{\tau}{\rightarrow} S^{\prime}$ where

$$
S^{\prime}=\left(\mathcal{E} ;\left\{\operatorname{out}\left(u,[v]_{i}\right) \cdot Q_{S}\right\} \uplus \mathcal{Q}_{S} ; \Phi_{S} ; \sigma_{S}\right) .
$$

Hence, we have that $B \stackrel{\tau}{\rightarrow}$ bi $B^{\prime}$ with $\mathrm{fst}\left(B^{\prime}\right)=S^{\prime}$, and $\operatorname{snd}\left(B^{\prime}\right)=D^{\prime}$. We also have that $D^{\prime}=\delta\left(S^{\prime}\right)$.

Case of the rule ELSE. This case is similar to the previous one.

Case of the rule ASSGN. In such a case, we have that $\mathcal{E}_{D}^{\prime}=$ $\mathcal{E}_{D}, \Phi_{D}^{\prime}=\Phi_{D}, \mathcal{P}_{D}=\left\{\left[x:=\delta_{\gamma}\left([v]_{i}\right)\right] . \delta(Q)\right\} \uplus \delta\left(\mathcal{Q}_{S}\right), \mathcal{P}_{D}^{\prime}=$ $\{\delta(Q)\} \uplus \delta\left(\mathcal{Q}_{S}\right), \sigma_{D}^{\prime}=\sigma_{D} \cup\left\{x \mapsto \delta_{\gamma}\left([v]_{i}\right) \sigma_{D}\right\}$, and $\ell=$ $\tau$ where $\gamma \in\{\alpha, \beta\}$ with $i \in \gamma$. We have also that $\sigma_{D} \vDash$ test $_{i}\left(\delta\left([v]_{i}\right)\right)$ and $\sigma_{S} \vDash \operatorname{test}_{i}\left([v]_{i}\right)$. Hence, we have that:

$$
S=\left(\mathcal{E} ;\left\{\left[x:=[v]_{i}\right] . Q\right\} \uplus \mathcal{Q}_{S} ; \Phi_{S} ; \sigma_{S}\right)
$$

with $\Phi_{D \downarrow}=\delta\left(\Phi_{S \downarrow}\right)$, and $\sigma_{D \downarrow}=\delta\left(\sigma_{S \downarrow} \downarrow\right)$.

Hence, we have that $S \stackrel{\tau}{\rightarrow} S^{\prime}$ where:

$$
S^{\prime}=\left(\mathcal{E} ;\{Q\} \uplus \mathcal{Q}_{S} ; \Phi_{S} ; \sigma_{S} \cup\left\{x \mapsto[v]_{i} \sigma_{S}\right\}\right) .
$$

Hence, we have that $B \stackrel{\tau}{\rightarrow}$ bi $B^{\prime}$ with $\mathrm{fst}\left(B^{\prime}\right)=S^{\prime}$ and snd $\left(B^{\prime}\right)=D^{\prime}$. It remains to show that $D^{\prime}=\delta\left(S^{\prime}\right)$, i.e.

$$
\left(\delta_{\gamma}\left([v]_{i}\right) \sigma_{D}\right) \downarrow=\delta_{\gamma}\left([v]_{i} \sigma_{S \downarrow}\right) .
$$

This can be done as in the case of the rule OUT-T.

Case of the rule CoMM. In such a case, we have that $\mathcal{P}_{D}=$ $\left\{\operatorname{out}\left(c, \delta_{\gamma}\left([u]_{i}\right)\right)^{i} . \delta\left(Q_{1}\right) ; \operatorname{in}(c, x)^{i^{\prime}} . \delta\left(Q_{2}\right)\right\} \uplus \delta\left(\mathcal{Q}_{S}\right), \mathcal{E}_{D}^{\prime}=$ $\mathcal{E}_{D}, \Phi_{D}^{\prime}=\Phi_{D}, \sigma_{D}^{\prime}=\sigma_{D} \cup\left\{x \mapsto \delta_{\gamma}\left([u]_{i}\right) \sigma_{D}\right\}$, and $\ell=$ $\tau$. Moreover, we have that $\sigma_{D} \vDash \delta_{\gamma}\left(\operatorname{test}_{i}\left([u]_{i}\right)\right)$ and $\sigma_{S} \vDash$ test $_{i}\left([v]_{i}\right)$ where $\gamma \in\{\alpha, \beta\}$ such that $i \in \gamma$. Hence, we have that $S$ is equal to

$$
\left(\mathcal{E} ;\left\{\operatorname{out}\left(c,[u]_{i}\right) \cdot Q_{1} ; \operatorname{in}(c, x) \cdot Q_{2}\right\} \uplus \mathcal{Q}_{S} ; \Phi_{S} ; \sigma_{S}\right)
$$

with $\Phi_{D \downarrow}=\delta\left(\Phi_{S \downarrow}\right)$, and $\sigma_{D \downarrow}=\delta\left(\sigma_{S \downarrow}\right)$.

Let $\gamma^{\prime} \in\{\alpha, \beta\}$ such that $i^{\prime} \in \gamma^{\prime}$. Hence, we have that $S \stackrel{\tau}{\rightarrow} S^{\prime}$ where $S^{\prime}$ is equal to:

$$
\left(\mathcal{E} ;\left\{Q_{1} ; Q_{2}\right\} \uplus \mathcal{Q}_{S} ; \Phi_{S} ; \sigma_{S} \cup\left\{\left(x \mapsto[u]_{i} \sigma_{S}\right)^{i^{\prime}}\right\}\right) .
$$

Hence, we have that $B \stackrel{\tau}{\rightarrow} B^{\prime}$ for some biprocess $B^{\prime}$ such that $\operatorname{fst}\left(B^{\prime}\right)=S^{\prime}$ and $\operatorname{snd}\left(B^{\prime}\right)=D^{\prime}$. It remains to show that $D^{\prime}=\delta\left(S^{\prime}\right)$, i.e.

$$
\left(\delta_{\gamma}\left([u]_{i}\right) \sigma_{D}\right) \downarrow=\delta_{\gamma^{\prime}}\left([u]_{i} \sigma_{S \downarrow}\right)
$$

If $\gamma=\gamma^{\prime}$, then this can be done as in the previous cases. Otherwise, since names can only be shared through assignations, and assignations only concern variables/terms of base type, we necessarily have that $c \notin \mathcal{E}$. Hence, we have that $D \stackrel{\nu w_{n} \text {.out }\left(c, w_{n}\right)}{\longrightarrow} D_{\text {out }}$ where $D_{\text {out }}$ is equal to:

$$
\left(\mathcal{E}_{D} ;\left\{\delta\left(Q_{1}\right) ; \operatorname{in}(c, x) . \delta\left(Q_{2}\right)\right\} \uplus \mathcal{Q}_{S} ; \Phi_{D} \cup\left\{w_{n} \triangleright \delta_{\gamma}\left([u]_{i}\right) \sigma_{D}\right\} ; \sigma_{D}\right)
$$

Note that $\left(\rho_{\alpha}, \rho_{\beta}\right)$ is still compatible with $D_{\text {out }}$. We would like to apply Lemma 53 with $M=w_{n}$ on the frame of $D_{\text {out }}$ which requires an hypothesis of non deductibility of the shared key. For these, we will rely on our hypothesis that $D_{0}$ does not reveal the values of his assignment variables w.r.t. $\left(\rho_{\alpha}, \rho_{\beta}\right)$ : 
Let $\Phi_{D}^{\prime}=\Phi_{D} \cup\left\{w_{n} \triangleright \delta_{\gamma}\left([u]_{i}\right) \sigma_{D}\right\}$. We already proved our induction result for the rule OUT-T. Hence, we deduce that there exists $S_{\text {out }}$ such that $S \stackrel{\nu w_{n} \text {.out }\left(c, w_{n}\right)}{\longrightarrow} S_{\text {out }}$ where $\left.S_{\text {out }}=\left(\mathcal{E} ; \mathcal{P}_{S}^{\prime} ; \Phi_{S}^{\prime} ; \sigma_{S}\right), \Phi_{S}^{\prime}=\Phi_{S} \cup\left\{w_{n} \triangleright[u]_{i}\right) \sigma_{S}\right\}$. Moreover, it implies that $\left.\Phi_{D}^{\prime} \downarrow=\delta\left(\Phi_{S}^{\prime} \downarrow\right\}\right)$ and $\sigma_{D} \downarrow=\delta\left(\sigma_{S} \downarrow\right)$. As mentioned, by hypothesis, we know that $D_{0}$ does not reveal the values of its assignments w.r.t. $\left(\rho_{\alpha}, \rho_{\beta}\right)$. Hence, for all assignment variable $x$ of color $\alpha$ (resp. $\beta$ ) in $\operatorname{dom}\left(\sigma_{D}\right)$, for all key $\in\left\{k, \operatorname{pk}(k), \operatorname{vk}(k) \mid k=x \sigma_{D} \vee k=x \rho_{\alpha}\right.$ (resp. $\left.\left.x \rho_{\beta}\right)\right\}$, key is not deducible from new $\mathcal{E} . \Phi_{D}^{\prime}$. We denote $K$ this set. Let $K_{S}=\left\{t, \mathrm{pk}(t), \mathrm{vk}(t) \mid t \in \operatorname{dom}\left(\rho_{\alpha}^{+}\right) \cup\right.$ $\operatorname{dom}\left(\rho_{\beta}^{+}\right), t$ ground $\}$. Since $\sigma_{D} \downarrow=\delta\left(\sigma_{S} \downarrow\right)$, and by definition of $\rho_{\alpha}^{+}$and $\rho_{\beta}^{+}$, we deduce that $K=\delta_{\alpha}\left(K_{S}\right) \cup \delta_{\beta}\left(K_{S}\right)$. We have also that $\Phi_{D}^{\prime} \downarrow=\delta\left(\Phi_{S}^{\prime} \downarrow\right)$. Hence, we can now apply Lemma 53 with $M=w_{n}$ and so we deduce that $\delta_{\gamma}\left([u]_{i} \sigma_{S} \downarrow\right)=\delta_{\gamma^{\prime}}\left([u]_{i} \sigma_{S} \downarrow\right)$. Hence, we can conclude as in the previous case.

Case of the rule PAR. It is easy to see that the result holds for this case.

Note that the rules NEW and REPL can not be triggered since the processes under study do not contain bounded names and replication.

Theorem 31: Let $P$ be a plain colored process as described above, and $B_{0}$ be an extended colored biprocess such that:

- $S_{0}=\left(\mathcal{E}_{\alpha} \uplus \mathcal{E}_{\beta} \uplus \mathcal{E}_{0} ; \llbracket P \rrbracket ; \emptyset ; \emptyset\right) \stackrel{\text { def }}{=} \mathrm{fst}\left(B_{0}\right)$,

- $D_{0}=\left(\mathcal{E}_{\alpha} \uplus \mathcal{E}_{\beta} \uplus \mathcal{E}_{0} ; P_{D} ; \emptyset ; \emptyset\right) \stackrel{\text { def }}{=} \operatorname{snd}\left(B_{0}\right)$, and

- $P_{D}=\delta_{\rho_{\alpha}, \rho_{\beta}}(\llbracket P \rrbracket)$ for some $\left(\rho_{\alpha}, \rho_{\beta}\right)$ compatible with $D_{0}$, and

- $D_{0}$ does not reveal its assignments w.r.t. $\left(\rho_{\alpha}, \rho_{\beta}\right)$.

We have that:

1) For any extended process $S=\left(\mathcal{E}_{S} ; \mathcal{P}_{S} ; \Phi_{S} ; \sigma_{S}\right)$ such that $S_{0} \stackrel{\text { tr }}{\Longrightarrow} S$ with $\left(\rho_{\alpha}, \rho_{\beta}\right)$ compatible with $S$, there exists a biprocess $B$ and an extended process $D=$ $\left(\mathcal{E}_{D} ; \mathcal{P}_{D} ; \Phi_{D} ; \sigma_{D}\right)$ such that $B_{0} \stackrel{\text { tr }}{\Longrightarrow}$ bi $B$, fst $(B)=S$, $\operatorname{snd}(B)=D$, and new $\mathcal{E}_{S} . \Phi_{S} \sim$ new $\mathcal{E}_{D} . \Phi_{D}$.

2) For any extended process $D=\left(\mathcal{E}_{D} ; \mathcal{P}_{D} ; \Phi_{D} ; \sigma_{D}\right)$ such that $D_{0} \stackrel{\text { tr }}{\Longrightarrow} D$ with $\left(\rho_{\alpha}, \rho_{\beta}\right)$ compatible with $D$, there exists a biprocess $B$ and an extended process $S=\left(\mathcal{E}_{S} ; \mathcal{P}_{S} ; \Phi_{S} ; \sigma_{S}\right)$ such that $B_{0} \stackrel{\text { tr }}{\Longrightarrow}$ bi $B$, fst $(B)=S$, $\operatorname{snd}(B)=D$, and new $\mathcal{E}_{S} . \Phi_{S} \sim$ new $\mathcal{E}_{D} . \Phi_{D}$.

Proof: We prove the two items separately.

1) The first item is actually a direct consequence of Proposition 56. We rely on Corollary 55 and the fact that $D=\delta(S)$ to establish that:

$$
\text { new } \mathcal{E}_{S} \cdot \Phi_{S} \sim \text { new } \mathcal{E}_{D} \cdot \Phi_{D} .
$$

2) The second item is actually a direct consequence of Proposition 57. We rely on Corollary 55, Corollary 54 and the fact that $D=\delta(S)$ to establish that:

$$
\text { new } \mathcal{E}_{S} . \Phi_{S} \sim \text { new } \mathcal{E}_{D} \cdot \Phi_{D} .
$$

This concludes the proof of the theorem.

\section{APPENDIX D \\ PARALLEL COMPOSITION}

The goal of this section is to prove the results stated in Section IV-A. We prove a slightly improved version of Theorem 15 assuming that composition contexts may contain several holes. To prove these composition results, we will rely on Theorem 31, and for this we have to explain how to get rid of the replications, and the new instructions (see Section D-A). We have also to rewrite the process to ensure that names are shared via assignment variables only (see Section D-B).

\section{A. Unfolding a biprocess}

Given an extended process $A=(\mathcal{E} ; \mathcal{P} ; \Phi)$ where $\mathcal{P}$ may contain name restrictions and replications, the idea is to unfold the replications and to gather together all the restricted names in the set $\mathcal{E}$. Of course, it is not possible to apply such a transformation and to preserve the set of possible traces. However, given a specific trace issued from $A$, it is possible to compute an unfolding of $A$ that will exhibit this specific trace. The converse is also true, any trace issued from an unfolding of $A$ will correspond to a trace of $A$. Thus, the process $A$ and all its possible unfoldings will exhibit exactly the same set of traces. We define this notion directly on biprocesses.

Definition 58: Let $A=(\mathcal{E} ; \mathcal{P} ; \Phi)$ be an extended biprocess. We define the $n^{\text {th }}$ unfolding of $A$, denote by $\operatorname{Unf}_{n}(A)$, the biprocess $\left(\mathcal{E} \uplus \mathcal{E}_{n} ; \mathcal{P}_{n} ; \Phi\right)$ obtained from $A$ by replacing in $\mathcal{P}$ each instance of $! Q$ with $n$ instances of $Q$ (applying $\alpha$ renaming to ensure name and variable distinctness), and then removing the new operations from the resulting process. These names are then put in the set $\mathcal{E}_{n}$ and added in the first component of the extended process.

The link between an extended biprocess and its unfoldings is stated in Lemma 59.

Lemma 59: Let $A=(\mathcal{E} ; \mathcal{P} ; \Phi)$ be an extended biprocess. The biprocess $A$ is in diff-equivalence if, and only if, $\operatorname{Unf}_{n}(A)$ is in diff-equivalence for any $n \in \mathbb{N}$.

\section{B. Sharing names via assignments}

In Theorem 31, one can note that processes may only share data through assignment variables. This is not a real limitation since a name that is shared via the composition context can be assigned to an assignment variable by one process and used by the other through the assignment variables. Below, we describe this transformation that actually preserves diff-equivalence of a biprocess.

Let $A=(\mathcal{E} ; \mathcal{P} ; \Phi)$ be an extended colored (with colors in $\{1, \ldots, p\}=\alpha \uplus \beta)$ biprocess that does not contain any name restriction nor replication in $\mathcal{P}$. Let $K=k_{1}, \ldots, k_{\ell}$ be a sequence of names (of base type) in $\mathcal{E}$ that contains at least all the names occurring in both type of actions - in actions colored $\alpha$ as well as in actions colored $\beta$ (intuitively $k_{1}, \ldots, k_{\ell}$ are the names shared by the two processes we want to compose). Since we work with a biprocess, we do this transformation simulatenously on both sides. We do this each time the transformation is required by one side of the biprocess. Actually, 
when we will apply this transformation, the right-hand side will correspond to the disjoint case, whereas the left-hand side will correspond to the shared case, and all the transformations will arise because of the left-hand side.

Let $Z=z_{1}^{\alpha}, \ldots, z_{\ell}^{\alpha}$ be a sequence of fresh variables, and $i \in \alpha$. We denote by $A s_{Z:=\mathcal{K}}^{i}(A)$ the extended biprocess $\left(\mathcal{E} ; P_{\text {ass }} ; \Phi\right)$ where $P_{\text {ass }}$ is defined as follows:

$$
P_{\text {ass }}=\left[z_{1}^{\alpha}:=k_{1}\right]^{i} \ldots . .\left[z_{\ell}^{\alpha}:=k_{\ell}\right]^{i} \cdot\left(\left.\right|_{P \in \mathcal{P}} P \rho_{\beta}\right)
$$

where $\rho_{\beta}$ replaces each occurrence of the name $k_{j}(1 \leq j \leq \ell)$ that occurs in an action $\beta$-colored by its associated assignment variable $z_{j}^{\alpha}(1 \leq j \leq \ell)$. Note that the replacement $\rho_{\beta}$ will not affect the process corresponding to the disjoint case.

Note that in the definition above, the $\alpha$-colored process will assign the shared names into assignment variables whereas the $\beta$-colored process will simply use those variables instead of the corresponding names. This choice is arbitrary and the roles played by $\alpha$ and $\beta$ can be swapped. Again, this transformation preserves equivalence. This result is stated below in Lemma 60.

Lemma 60: Let $A=(\mathcal{E} ; \mathcal{P} ; \Phi)$ and $A s s_{Z:=\mathcal{K}}^{i}(A)$ be two extended biprocesses as described above. We have that $A$ is in diff-equivalence if, and only if, $\operatorname{Ass}_{Z:=\mathcal{K}}^{i}(A)$ is in diffequivalence.

\section{Composing trace equivalence}

The theorem we want to prove is stated below. Note that, this theorem differs from the one stated in the main body of the paper since we work in a slightly more general setting.

We denote by $\Sigma_{0}^{c}=\{$ senc, aenc, sign, pk, vk, \langle\rangle$\}$, i.e. the constructors of the common signature $\Sigma_{0}$. We consider composition contexts that may contain several holes. They are formally defined as follows:

Definition 61: A composition context $C$ is defined by the following grammar where $n$ is a name of base type.

$$
C, C_{1}, C_{2}:=\_\mid \text {new } n . C|! C| C_{1} \mid C_{2}
$$

We only allow names of base type (typically keys) to be shared between processes through the composition context. In particular, they are not allowed to share a private channel even if each process can used its own private channels to communicate internally. We also suppose w.l.o.g. that names occurring in $C$ are distinct. A composition context may contain several holes. We can index them to avoid confusion. We write $C\left[P_{1}, \ldots, P_{\ell}\right]$ (or shortly $C[\bar{P}]$ ) the process obtained by filling the $i^{\text {th }}$ hole with the process $P_{i}$ (or the $i^{\text {th }}$ process of the sequence $\bar{P}$ ).

We use the notation $\bar{P} \mid \bar{Q}$ to represent the sequence of processes obtained by putting in parallel the processes of the sequences $\bar{P}$ and $\bar{Q}$ componentwise.

Parallel composition between tagged processes can only be achieved assuming that the shared keys are not revealed. Indeed, if the security of $P$ is ensure through the secrecy of the shared key $k$, there is no way to guarantee that $P$ is still secure in an environment where another process $Q$ running in parallel will reveal this key.

Since, we consider a common signature $\Sigma_{0}$ and composition contexts with several holes, we have to generalize a bit the notion of revealing a shared key stated in the body of the paper. We have to take into account public keys and verification keys.

Definition 62: Let $C$ be a composition context, $A$ be an extended process of the form $\left(\mathcal{E} ; C\left[P_{1}, \ldots, P_{\ell}\right] ; \Phi ; \sigma\right)$, and key $\in\{n, \operatorname{pk}(n), \operatorname{vk}(n) \mid n$ occurs in $C\}$. We say that the extended process $A$ reveals the key key when:

- $\left(\mathcal{E} \cup\{s\} ; C\left[P_{1}^{+}, \ldots, P_{\ell}^{+}\right] ; \Phi ; \sigma\right) \stackrel{w}{\Rightarrow}\left(\mathcal{E}^{\prime} ; \mathcal{P}^{\prime} ; \Phi^{\prime} ; \sigma^{\prime}\right)$ with $P_{i_{0}}^{+} \stackrel{\text { def }}{=} P_{i_{0}} \mid$ in $(c, x)$. if $x=$ key then out $(c, s)$ and $P_{i}^{+} \stackrel{\text { def }}{=} P_{i}$ if $i \neq i_{0}$; and

- $M \Phi^{\prime}=\mathrm{E} s$ for some $M$ such that $f v(M) \subseteq \operatorname{dom}\left(\Phi^{\prime}\right)$ and $f n(M) \cap \mathcal{E}^{\prime}=\emptyset$

where $c$ is a fresh public channel name, $s$ is a fresh name of base type, and the $i_{0}$ th hole of $C$ is in the scope of "new fn(key)".

Definition 63: Let $C$ be a composition context and $\mathcal{E}_{0}$ be a finite set of names of base type. Let $\bar{P}$ and $\bar{Q}$ be two sequences of plain processes together with their frames $\Phi$ and $\Psi$. We say that $\bar{P} / \Phi$ and $\bar{Q} / \Psi$ are composable under $\mathcal{E}_{0}$ and $C$ when:

1) $\bar{P}$ (resp. $\bar{Q}$ ) are built over $\Sigma_{\alpha} \cup \Sigma_{0}$ (resp. $\Sigma_{\beta} \cup \Sigma_{0}$ ), whereas $\Phi$ (resp. $\Psi$ ) are built over $\Sigma_{\alpha} \cup\{$ pk, vk\} (resp. $\Sigma_{\beta} \cup\{\mathrm{pk}, \mathrm{vk}\}$ ), $\Sigma_{\alpha} \cap \Sigma_{\beta}=\emptyset$, and $\bar{P}$ (resp. $\bar{Q}$ ) is tagged;

2) $f v(\bar{P})=f v(\bar{Q})=\emptyset$, and $\operatorname{dom}(\Phi) \cap \operatorname{dom}(\Psi)=\emptyset$.

3) $\mathcal{E}_{0} \cap(f n(C[\bar{P}]) \cup f n(\Phi)) \cap(f n(C[\bar{Q}]) \cup f n(\Psi))=\emptyset$;

4) $\left(\mathcal{E}_{0} ; C[\bar{P}] ; \Phi\right)\left(\right.$ resp. $\left.\left(\mathcal{E}_{0} ; C[\bar{Q}] ; \Psi\right)\right)$ does not reveal any key in:

$$
\{n, \operatorname{pk}(n), \operatorname{vk}(n) \mid n \text { occurs in } f n(\bar{P}) \cap f n(\bar{Q}) \cap b n(C)\} .
$$

This notion is extended as expected to biprocesses requiring that $\mathrm{fst}(\bar{P}) / \mathrm{fst}(\Phi)$ and $\mathrm{fst}(\bar{Q}) / \mathrm{fst}(\Psi)$, as well as $\operatorname{snd}(\bar{P}) / \operatorname{snd}(\Phi)$ and $\operatorname{snd}(\bar{Q}) / \operatorname{snd}(\Psi)$, are composable.

Theorem 64: Let $C$ be a composition context, and $\mathcal{E}_{0}$ be a finite set of names of base type. Let $\bar{P}$ (resp. $\bar{Q}$ ) be a sequence of plain biprocesses together with its frame $\Phi$ (resp. $\Psi$ ), and assume that $\bar{P} / \Phi$ and $\bar{Q} / \Psi$ are composable under $\mathcal{E}_{0}$ and $C$.

If $\left(\mathcal{E}_{0} ; C[\bar{P}] ; \Phi\right)$ and $\left(\mathcal{E}_{0} ; C[\bar{Q}] ; \Psi\right)$ satisfy diff-equivalence (resp. trace equivalence), then $\left(\mathcal{E}_{0} ; C[\bar{P} \mid \bar{Q}] ; \Phi \uplus \Psi\right)$ satisfies diff-equivalence (resp. trace equivalence).

Proof: According to our hypothesis, $\bar{P}$ and $\bar{Q}$ are both tagged hence there exists two sequences of colored plain processes $\overline{P_{t}}$ and $\overline{Q_{t}}$ such that $\llbracket \overline{P_{t}} \rrbracket=\bar{P}$ and $\llbracket \overline{Q_{t}} \rrbracket=\bar{Q}$. Moreover, we can split the set of names $\mathcal{E}_{0}$ into two disjoint sets $\mathcal{E}_{P}$ and $\mathcal{E}_{Q}$ depending on whether the name occurs in $\bar{P} / \Phi$ or $\bar{Q} / \Psi$.

Let $S=\left(\mathcal{E}_{0} ; C[\bar{P} \mid \bar{Q}] ; \Phi\right)$. Our goal is to show that $S$ satisfies diff-equivalence (resp trace equivalence). By hypothesis, we actually have that $\left(\mathcal{E}_{P} ; C[\bar{P}] ; \Phi\right)$, and $\left(\mathcal{E}_{Q} ; C[\bar{Q}] ; \Psi\right)$ satisfy diff-equivalence (resp trace equivalence). Let $D=$ $\left(\mathcal{E}_{P} \uplus \mathcal{E}_{Q} ; C[\bar{P}] \mid C[\bar{Q}] ; \Phi \uplus \Psi\right)$ (modulo some $\alpha$-renaming to 
ensure name and variable distinctness of the resulting process). Since the two processes that are composed in parallel do not share any data, we have that $D$ satisfies diff-equivalence (resp trace equivalence). In order to conclude that $S$ satisfies diff-equivalence (resp trace equivalence), we will show that fst $(S) \approx_{\text {diff }} \operatorname{fst}(D)$ and $\operatorname{snd}(S) \approx_{\text {diff }} \operatorname{snd}(D)$ relying on Theorem 31.

Let $B_{1}$ be the biprocess obtained by forming a biprocess with $\mathrm{fst}(S)$ and $\mathrm{fst}(D)$. Even if the two processes do not have exactly the same structure, this can be achieved by introducing some new instructions that will not be used in fst $(S)$. Relying on Lemma 59, we have that $B_{1}$ is in diff-equivalence if and only if $\operatorname{Unf}_{n}\left(B_{1}\right)$ is in diff-equivalence for any $n \in \mathbb{N}$. Let $n_{0} \in \mathbb{N}$. We transform the biprocess $\operatorname{Unf}_{n_{0}}\left(B_{1}\right)$ to introduce assignment variables (and we may assume w.l.o.g. that the processes under study do not rely on any assignment variables, thus the resulting process will only contain the assignment variables introduced by our transformation), namely $z_{1}^{\alpha}, \ldots, z_{\ell}^{\alpha}$. This leads us to another biprocess and this transformation still preserves diff-equivalence as stated in Lemma 60. Note that, on the right-hand side of the biprocess (the disjoint case), the assignments variables are assigned to names that do not occur in any action colored $\beta$. In order to apply our Theorem 31, we perform a last transformation on this biprocess that consists in replacing the elements that occur inside the frame by output actions (colored with $\alpha$ or $\beta$ depending on its origin) in front of the biprocess. This last transformation preserves also diff-equivalence. We finally consider $\mathcal{E}_{\alpha}=\emptyset$, and $\mathcal{E}_{\beta}=\left\{k_{1}^{\alpha}, \ldots, k_{\ell}^{\alpha}\right\}$ a set of fresh names, and we add these two sets of names to the set of $\mathcal{E}_{0}$ (first argument of the biprocess). Now, it remains to show that this resulting biprocess $B_{1}^{\prime}$ is in diff-equivalence. For this, we rely on Theorem 31. Let $\rho_{\alpha}$ be such that $\operatorname{dom}\left(\rho_{\alpha}\right)=\emptyset$, and $\rho_{\beta}$ be such that $\operatorname{dom}\left(\rho_{\beta}\right)=\left\{z_{1}^{\alpha}, \ldots, z_{\ell}^{\alpha}\right\}$, and $z_{j}^{\alpha} \rho_{\beta}=k_{j}^{\alpha}$ for $j \in\{1, \ldots, \ell\}$. Actually, we have that $D_{1}^{\prime}=\delta\left(S_{1}^{\prime}\right)$ where $S_{1}^{\prime}=\operatorname{fst}\left(B_{1}^{\prime}\right)$ and $D_{1}^{\prime}=\operatorname{snd}\left(B_{1}^{\prime}\right)$, and for all possible executions of $S_{1}^{\prime}$ or $D_{1}^{\prime}$, compatibility will be satisfied. Indeed, by construction, we know that all the assignment variables (remember that all the assignments occurring in the process have been introduced by our transformation) will be assigned to distinct names. Now, to satisfy all the requirements needed to apply Theorem 31, it remains to establish the non-deducibility of the keys.

By hypothesis, $\left(\mathcal{E}_{0} ; C[\bar{P}] ; \Phi\right)$ and $\left(\mathcal{E}_{0} ; C[\bar{Q}] ; \Psi\right)$ do not reveal $k, \operatorname{pk}(k)$, or vk $(k)$ for any $k \in f n(\bar{P}) \cap f n(\bar{Q}) \cap b n(C)$. Hence, we deduce that fst $(D)$ (parallel composition - disjoint case) does not reveal $k, \operatorname{pk}(k)$, or $\mathrm{vk}(k)$ for any $k \in f n(\bar{P}) \cap$ $f n(\bar{Q}) \cap b n(C)$.

Note that we want to apply Theorem 31 on $S_{1}^{\prime}$ and $D_{1}^{\prime}$ and not on $S_{1}$ and $D_{1}$. However, we built $D_{1}^{\prime}$ by unfolding $D_{1}$ and introducing assignment variables. First, note that these transformations preserve deducibility. Moreover, secrecy of $k$, $\mathrm{pk}(k)$, or $\mathrm{vk}(k)$ for any $k \in f n(\bar{P}) \cap f n(\bar{Q}) \cap b n(C)$ actually implies that $D_{1}^{\prime}$ does not reveal its assignments w.r.t. $\left(\rho_{\alpha}, \rho_{\beta}\right)$. This allows us to apply Theorem 31 and so to conclude.

\section{Composing reachability}

We now prove a variant of Corollary 16 considering our slightly more general setting.

Corollary 65: Under the same hypotheses as Theorem 64 with processes instead of bioprocesses, and considering a name $s$ that occurs in $C$. If $\left(\mathcal{E}_{0} ; C[\bar{P}] ; \Phi\right)$ and $\left(\mathcal{E}_{0} ; C[\bar{Q}] ; \Psi\right)$ do not reveal $s$, then $\left(\mathcal{E}_{0} ; C[\bar{P} \mid \bar{Q}] ; \Phi \cap \Psi\right)$ does not reveal $s$.

Proof: The proof follows the same lines as the one for dealing with diff-equivalence and trace equivalence. In order to show that the process $S=\left(\mathcal{E}_{0} ; C[\bar{P} \mid \bar{Q}] ; \Phi \uplus \Psi\right)$ does not reveal $s$, we rely on the fact that the secrecy is preserved by parallel composition of "disjoint" processes. Thanks to our hypotheses, we have that $D=\left(\mathcal{E}_{0} ; C[\bar{P}] \mid C[\bar{Q}] ; \Phi \uplus \Psi\right)$ does not reveal $s$. Then, by applying Theorem 31 and more specifically the first bullet point of this theorem, we can deduce that for all $\left(\operatorname{tr}\right.$, new $\left.\mathcal{E}_{S} . \Phi_{S}\right) \in \operatorname{trace}(S)$, there exists a trace $\left(\operatorname{tr}\right.$, new $\left.\mathcal{E}_{D} . \Phi_{D}\right) \in \operatorname{trace}(D)$ such that new $\mathcal{E}_{S} . \Phi_{S} \sim$ new $\mathcal{E}_{D} . \Phi_{D}$. Since $D$ does not reveal $s$, we conclude that $S$ does not reveal $s$ too.

\section{APPENDIX E \\ SEQUENTIAL COMPOSITION}

In this section we prove Theorem 17. As for establishing parallel composition results, we will rely on Theorem 31 . This will require to unfold the processes under study, and to use assignment variables to share data. However, as already discussed in Section III, we also have to tackle some additional difficulties. In particular, to ensure the compatibility of the executions as required by Theorem 31 .

\section{A. Unfolding biprocesses and sharing names via assignments}

Unfolding the biprocesses for sequential composition follows the same principles as unfolding the biprocesses for parallel composition. However, we need to be more specific. In particular, we need to be able to easily talk about the replicated instances of a nonce after unfolding. We explain in this section how the unfolded biprocesses are built, and we introduce some notation that we will use throughout the entire section.

Example 66: Let $P=$ !new $k$.!new $n$.out $(c, \operatorname{senc}(n, k))$. The plain process

$$
\begin{array}{r|l}
\operatorname{out}(c, \operatorname{senc}(n[1,1], k[1])) \mid & \operatorname{out}(c, \operatorname{senc}(n[1,2], k[1])) \\
\mid \operatorname{out}(c, \operatorname{senc}(n[2,1], k[2])) & \operatorname{out}(c, \operatorname{senc}(n[2,2], k[2]))
\end{array}
$$

together with the set

$$
\mathcal{K}=\{k[1], k[2], n[1,1], n[1,2], n[2,1], n[2,2]\}
$$

will correspond to the 2-unfolding of $P$, denoted $\operatorname{Unf}_{2}(P)$. In this example, $k[1], k[2], n[1,1], \ldots, n[2,2]$ are considered as distinct names.

More generally, in such formalism, two names $n_{1}\left[i_{1}, \ldots, i_{p}\right]$ and $n_{2}\left[j_{1}, \ldots, j_{q}\right]$ are equal if, and only if, they are syntactically equal, i.e. $n_{1}=n_{2}, p=q$ and $i_{k}=j_{k}$ for each $k \in\{1 \ldots p\}$. We will use the same convention to represent the variables occurring in the processes. We will also extend 
this notation to processes. Thus $P\left[i_{1}, \ldots, i_{n}\right]$ will represent the instance of $P$ that correspond to the $i_{1}^{\text {th }}$ instance of the $1^{\text {st }}$ replication, $i_{2}^{\text {th }}$ instance of the $2^{\text {nd }}$ replication, etc.

Example 67: Going back to our previous example, we have that $\operatorname{Unf}_{2}(P)=(Q[1,1]|Q[1,2]| Q[2,1] \mid Q[2,2], \mathcal{K})$ where $Q[i, j]=\operatorname{out}(c, \operatorname{senc}(n[i, j], k[i]))$.

With such notation, we can now be much more precise on how our disjoint and shared processes are unfolded.

Following notation given in Theorem 17, we will consider the biprocesses:

1) $S=\left(\mathcal{E}_{0} ; C\left[P_{1}\left[Q_{1}\right] \mid P_{2}\left[Q_{2}\right]\right] ; \Phi \uplus \Psi\right)$, the so-called shared case;

2) $D^{\text {par }}=\left(\mathcal{E}_{0} ; C[P] \mid C[Q] ; \Phi \uplus \Psi\right)$, the so-called parallel disjoint case;

3) $D^{\text {seq }}=\left(\mathcal{E}_{0} ; \tilde{C}\left[P_{1}\left[\tilde{Q}_{1}\right] \mid P_{2}\left[\tilde{Q}_{2}\right]\right] ; \Phi \uplus \Psi\right)$ where $\tilde{C}$ is as $C$ but each name $n$ is duplicated $n / n^{Q}$ in order to ensure disjointness. The processes $\tilde{Q}_{1}$ and $\tilde{Q}_{2}$ are obtained from $Q_{1}$ and $Q_{2}$ by replacing each name $n$ occurring in $C$ by its copy $n^{Q}$. This represents the so-called sequential disjoint case.

Then, given a biprocess $B$ (typically one given above), we denote by $B_{n}$ its $n^{\text {th }}$ unfolding relying on the naming convention introduced in Example 66 and Example 67.

Using the notation introduced above, it should be clear that for each unfolding $n$ (with $n \in \mathbb{N}$ ), the biprocess that represents the parallel disjoint case, i.e. $D_{n}^{\text {par }}$ exhibits more behaviours than the biprocess that represents the sequential disjoint case, i.e. $D_{n}^{\text {seq }}$.

Lemma 68: If $D_{n}^{\text {par }}$ satisfies diff-equivalence then $D_{n}^{\text {seq }}$ satisfies diff-equivalence

As for parallel composition, once unfolding has been done, we get rid of names that are shared through the composition context using assignment variables. We denote these names $r_{1}, \ldots r_{p}$ and their associated assignment variables $z_{1}, \ldots, z_{p}$. We also get rid of the content of the frame by adding some outputs in front of the resulting process. Note that, we can assume w.l.o.g. that the only assignment instructions are those that occur in $P_{1}$ and $P_{2}$ to give a value to $x_{1}$ and $x_{2}$. Indeed, an assignment of the form $[x:=t]$ that is "local" to $P_{1} / P_{2}$ (or $Q_{1} / Q_{2}$ ) has the same effect as applying the substitution $x \mapsto t$ directly on the process. This additional hypothesis will help us ensure compatibility of all executions when applying Theorem 31.

Given a biprocess $B$, we will denote $B^{v}$ the biprocess resulting from the transformation described above. In particular, we will consider $S_{n}^{\mathrm{v}}$ the biprocess obtained by applying the transformation above on $S_{n}$ (the $n^{\text {th }}$ unfolding of the shared case), and also $D_{n}^{\text {vseq }}$ the biprocess obtained by applying the transformation on $D_{n}^{\text {seq }}$.

Again, it should be clear that these transformations preserve diff-equivalence.

Lemma 69: We have that:
1) $D_{n}^{\text {vseq }}$ satisfies diff-equivalence if, and only if, $D_{n}^{\text {seq }}$ satisfies diff-equivalence

2) $S_{n}^{\vee}$ satisfies diff-equivalence if, and only if, $S_{n}$ satisfies diff-equivalence

Relying on this transformation, by colouring actions of $P$ with $\alpha$, and actions of $Q$ with $\beta$, given an integer $n$ corresponding to the unfolding under study, and assuming that the hole of $C$ is under $m$ replications, we consider $\rho_{\alpha}$ such that $\operatorname{dom}\left(\rho_{\alpha}\right)=\emptyset$, and $\rho_{\beta}$ with

$$
\begin{aligned}
& \operatorname{dom}\left(\rho_{\beta}\right)=\left\{z_{1}, \ldots, z_{p}\right\} \\
& \quad \cup\left\{x_{1}\left[i_{1}, \ldots, i_{m}\right], x_{2}\left[i_{1}, \ldots, i_{m}\right] \mid 1 \leq i_{1}, \ldots, i_{m} \leq n\right\} \\
& \text { - } z_{i} \rho_{\beta}=r_{i} \text { for } 1 \leq i \leq p ; \\
& \text { - } \rho_{\beta}\left(x_{1}\left[i_{1}, \ldots, i_{m}\right]\right)=k\left[i_{1}, \ldots, i_{m}\right] \\
& \text { - } \rho_{\beta}\left(x_{2}\left[i_{1}, \ldots, i_{m}\right]\right)=k\left[i_{1}, \ldots, i_{m}\right] .
\end{aligned}
$$

In other words, we abstract each name shared via the composition context by a fresh one, i.e. $r_{i}$, and each term shared through the variables $x_{1}$ and $x_{2}$ are abstracted by a fresh name, a new one for each instance.

\section{B. Secrecy of the shared keys}

We now focus on the fourth condition of Theorem 31, i.e. we ensure that $D_{n}^{\text {vseq }}$ does not reveal the values of its assignments w.r.t. $\left(\rho_{\alpha}, \rho_{\beta}\right)$ as defined in Section E-A.

Lemma 70: Assume that $P_{1} / P_{2} / \Phi$ is a good key-exchange protocol under $\mathcal{E}_{0}$ and $C$. Assume also that $\left(\mathcal{E}_{0} ; C[Q] ; \Psi ; \emptyset\right)$ does not reveal any $k, \operatorname{pk}(k)$ and $\operatorname{vk}(k)$.

In such a case, we have that $D_{n}^{\text {vseq }}$ does not reveal the value of its assignment variables w.r.t. $\left(\rho_{\alpha}, \rho_{\beta}\right)$.

Proof: By Definition 11, $P_{1} / P_{2} / \Phi$ being a good keyexchange protocol under $\mathcal{E}_{0}$ and $C$ implies that $\left(\mathcal{E}_{0} ; P_{\text {good }} ; \Phi\right)$ does not reveal bad where $P_{\text {good }}$ is defined as follows:

$$
\begin{aligned}
& P_{\text {good }}=\text { new bad, } d .( \\
& C\left[\text { new } i d .\left(P_{1}\left[\operatorname{out}\left(d,\left\langle x_{1}, i d\right\rangle\right)\right] \mid P_{2}\left[\operatorname{out}\left(d,\left\langle x_{2}, i d\right\rangle\right)\right]\right)\right] \\
& \mid \operatorname{in}(d, x) \cdot \operatorname{in}(d, y) . \\
& \quad \text { if } \operatorname{proj}_{1}(x)=\operatorname{proj}_{1}(y) \wedge \operatorname{proj}_{2}(x) \neq \operatorname{proj}_{2}(y) \\
& \quad \text { then out }(c, b a d) \\
& \mid \text { in }(d, x) . \operatorname{in}(d, y) . \\
& \quad \text { if } \operatorname{proj}{ }_{1}(x) \neq \operatorname{proj}_{1}(y) \wedge \operatorname{proj}_{2}(x)=\operatorname{proj}_{2}(y) \\
& \quad \text { then out }(c, b a d) \\
& \mid \text { in }(d, x) . \operatorname{in}(c, z) . \\
& \quad \text { if } z \in\left\{\operatorname{proj}_{1}(x), \operatorname{pk}\left(\operatorname{proj}_{1}(x)\right), \operatorname{vk}\left(\operatorname{proj}_{1}(x)\right)\right\} \\
& \quad \text { then out }(c, b a d))
\end{aligned}
$$

In particular, it indicates that the secrecy of $x, \operatorname{pk}(x)$ and $\operatorname{vk}(x)$ is preserved, where $x$ is the value of any assignment variable. Then, the result is actually a direct consequence of the fact that secrecy is preserved through disjoint composition, and the transformations that are performed on the process (e.g. unfolding, adding of some assignments operations) also preserve secrecy. 


\section{Compatibility}

To use Theorem 31, a compatibility condition is required. As in the case of parallel composition, this property will be trivially satisfied for assignments that have been added by our transformation. However, more work is needed to deal with assignments present in the original processes, that is in our situation, assignments of the form $\left[x_{1}[\ldots]={ }_{-}\right]$and $\left[x_{2}[\ldots]=\right.$ - $]$ that come from the unfolding of the process $P_{1} / P_{2}$. The idea is that the abstractability property and the fact that $P_{1} / P_{2} / \Phi$ is a good key-exchange protocol will give us the required conditions to apply Theorem 31 .

The following lemma focuses on $P_{1} / P_{2} / \Phi$ being a good key-exchange protocol under $\mathcal{E}_{0}$ and $C$.

Lemma 71: Let $\left(\mathcal{E}_{0} ; C\left[P_{1}[0] \mid P_{2}[0]\right] ; \Phi ; \emptyset\right)$ be a process such that $P_{1} / P_{2} / \Phi$ is a good key-exchange protocol under $\mathcal{E}_{0}$ and $C$. Let $n$ be an integer, and $\left(\mathcal{E} ; \mathcal{P} ; \Phi^{\prime} ; \sigma\right)$ a process such that $\operatorname{fst}\left(D_{n}^{\text {seq }}\right) \stackrel{\text { tr }}{\Rightarrow}\left(\mathcal{E} ; \mathcal{P} ; \Phi^{\prime} ; \sigma\right)$. Let $i_{1}, j_{1}, \ldots, i_{m}, j_{m} \in \mathbb{N}$, and $q_{1}, q_{2} \in\{1,2\}$ such that $x_{q_{1}}\left[i_{1}, \ldots, i_{m}\right]$ and $x_{q_{2}}\left[j_{1}, \ldots\right.$, $\left.j_{m}\right]$ are in $\operatorname{dom}(\sigma)$. We have that:

$$
\begin{gathered}
x_{q_{1}}\left[i_{1}, \ldots, i_{m}\right] \sigma \downarrow=x_{q_{2}}\left[j_{1}, \ldots, j_{m}\right] \sigma \downarrow \\
\text { if, and only if, } \\
i_{p}=j_{p} \text { for all } 1 \leq p \leq m .
\end{gathered}
$$

A similar property holds for $\operatorname{snd}\left(D_{n}^{\text {seq }}\right)$.

Proof: By definition of $P_{1} / P_{2} / \Phi$ being a good keyexchange protocol under $\mathcal{E}_{0}$ and $C$ and since secrecy is preserved when considering disjoint composition, we have that $\left(\mathcal{E}_{0} ; P ; \Phi \uplus \Psi ; \emptyset\right)$ preserves the secrecy of bad where:

$$
\begin{aligned}
& P=\text { new bad.new } d .( \\
& \tilde{C}\left[\text { new } k \text {.new } i d .\left(P_{1}\left[\operatorname{out}\left(d,\left\langle x_{1}, i d\right\rangle\right) \cdot \tilde{Q}_{1}\left\{{ }^{k} / x_{1}\right\}\right]\right.\right. \\
& \left.\left.\qquad P_{2}\left[\operatorname{out}\left(d,\left\langle x_{2}, i d\right\rangle\right) \cdot \tilde{Q}_{2}\left\{{ }^{k} / x_{2}\right\}\right]\right)\right] \\
& \mid \operatorname{in}(d, x) \cdot \operatorname{in}(d, y) . \\
& \quad \text { if } \operatorname{proj}_{1}(x)=\operatorname{proj}_{1}(y) \wedge \operatorname{proj}_{2}(x) \neq \operatorname{proj}_{2}(y) \\
& \quad \text { then out }(c, b a d) \\
& \quad \text { elseif } \operatorname{proj}_{1}(x) \neq \operatorname{proj}_{1}(y) \wedge \operatorname{proj}_{2}(x)=\operatorname{proj}_{2}(y) \\
& \quad \text { then out }(c, b a d) \\
& \quad \quad
\end{aligned}
$$

Here, the notation $\tilde{C}, \tilde{Q}_{1}$, and $\tilde{Q}_{2}$ refer to the same renaming as the one used to define $D^{\text {seq }}$.

Let $n$ be an integer. Consider the $n^{\text {th }}$ unfolding of $D^{\text {seq }}$ as well as the $n^{\text {th }}$ unfolding of the process $P$ defined above. First, note that an output on channel $d$ is always of the form

$$
\operatorname{out}\left(d,\left\langle x_{j}\left[i_{1}, \ldots i_{m}\right], i d\left[i_{1}, \ldots i_{m}\right]\right\rangle\right) \text { with } j \in\{1,2\} \text {. }
$$

Let $\left(\mathcal{E} ; \mathcal{P} ; \Phi^{\prime} ; \sigma\right)$ be a process such that fst $\left(D_{n}^{\text {seq }}\right) \stackrel{\text { tr }}{\Rightarrow}\left(\mathcal{E} ; \mathcal{P} ; \Phi^{\prime} ; \sigma\right) \quad$ with $\quad x_{q_{1}}\left[i_{1}, \ldots, i_{m}\right] \quad$ and $x_{q_{2}}\left[j_{1}, \ldots, j_{m}\right]$ both in $\operatorname{dom}(\sigma)$. Moreover, assume that $x_{q_{1}}\left[i_{1}, \ldots, i_{m}\right] \sigma \downarrow=x_{q_{2}}\left[j_{1}, \ldots, j_{m}\right] \sigma \downarrow$. In such a case, it is easy to build a trace of $\left(\mathcal{E}_{0} ; P_{n} ; \Phi \uplus \Psi ; \emptyset\right)$ such that the pairs

- $\left\langle x_{q_{1}}\left[i_{1}, \ldots, i_{m}\right], i d\left[i_{1}, \ldots, i_{m}\right]\right\rangle$, and

- $\left\langle x_{q_{2}}\left[j_{1}, \ldots, j_{m}\right], i d\left[j_{1}, \ldots, j_{m}\right]\right\rangle$

are outputted on channel $d$. Since the hole in $P_{q_{1}}$ (resp. $P_{q_{2}}$ ) is not in the scope of a replication, we deduce that these pairs can only be outputted once. We have seen that such a process preserves the secrecy of $b a d$, and thus we deduce that $\left(i_{1}, \ldots, i_{p}\right)=\left(j_{1}, \ldots, j_{p}\right)$.

Now, relying on the fact that $\left(\mathcal{E}_{0} ; P_{n} ; \Phi \uplus \Psi ; \emptyset\right)$ preserves the secrecy of bad, and more precisely on the fact that the following instructions are part of the process:

$$
\begin{aligned}
& \text { In }(d, x) \cdot \text { in }(d, y) . \\
& \quad \ldots \\
& \quad \text { else if } \operatorname{proj}_{1}(x) \neq \operatorname{proj}_{1}(y) \wedge \operatorname{proj}_{2}(x)=\operatorname{proj}_{2}(y) \\
& \text { then out }(c, \text { bad })
\end{aligned}
$$

we deduce that $\left(i_{1}, \ldots, i_{p}\right)=\left(j_{1}, \ldots, j_{p}\right)$ implies that $i d\left[i_{1}, \ldots, i_{m}\right]=i d\left[j_{1}, \ldots, j_{m}\right]$ and so we deduce that $x_{q_{1}}\left[i_{1}, \ldots, i_{m}\right] \sigma \downarrow=x_{q_{2}}\left[j_{1}, \ldots, j_{m}\right] \sigma \downarrow$.

Now, regarding assingment variables, and in particular the different instances of $x_{1}$ and $x_{2}$, it remains to show that the values assigned to these variables will be rooted in the right signature. We proceed in two steps. First, we discard terms rooted with a symbol in $\{\mathrm{pk}, \mathrm{vk},\langle\rangle\}$ (Lemma 74), and then we show that it is actually rooted in the right signature (Lemma 75).

Definition 72: We say that a process $P$ satisfies the $a b$ stractability property if for all $P \stackrel{\text { tr }}{\Rightarrow}(\mathcal{E} ; \mathcal{P} ; \Phi ; \sigma)$, for all assignment variable $x \in \operatorname{dom}(\sigma), \operatorname{root}(x \sigma \downarrow) \notin\{\mathrm{pk}, \mathrm{vk},\langle\rangle\}$.

This property is important for our composition to hold.

Example 73: Let $P_{i}=\left[x_{i}:=\left\langle k_{1}, k_{2}\right\rangle\right]$, and $Q_{i}=$ if $x_{i}=\left\langle\operatorname{proj}_{1}\left(x_{i}\right), \operatorname{proj}_{2}\left(x_{i}\right)\right\rangle$ then out $\left(c, i d_{i}\right)$. Let $C=$

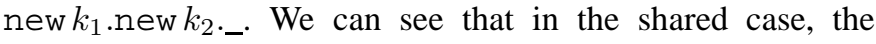
branch THEN of the process $Q_{i}$ will be executed whereas when considering in isolation the process $C\left[\right.$ new $k .\left(Q_{1}\left\{x_{1} \mapsto k\right\}\right)$ $\left.\left.Q_{2}\left\{x_{2} \mapsto k\right\}\right)\right]$ will not exhibit a similar behaviour.

Intuitively, we say that a value of an assignment variable can be abstracted if it is not a pair, a public key or verification key. This is due to the fact that those three primitives are not tagged and so can be used by processes of any colour.

Lemma 74: Let $\left(\mathcal{E}_{0} ; C\left[P_{1}[0] \mid P_{2}[0]\right] ; \Phi ; \emptyset\right)$ be a process satisfying the abstractability property. We have that $D^{\text {vseq }}$ satisfies the abstractability property.

Proof: First of all, unfolding the process $\left(\mathcal{E}_{0} ; C\left[P_{1}[0] \mid P_{2}[0]\right] ; \Phi ; \emptyset\right)$ preserves the abstractability property. Moreover, the transformation that transforms a process $B$ into a process $B^{\vee}$ preserves the abstractability property. Thus, to show that $D^{\text {vseq }}$ satisfies the abstractability property, it only remains to show that this property is preserved by disjoint composition assuming that the process we want to compose does not introduce new assignments (note that this is the case of $Q_{1} / Q_{2}$ ).

In fact, part of the process brought by $Q_{1} / Q_{2}$ can be viewed as a process executed by the attacker. Thus, for all $D^{\text {seq }} \stackrel{\text { tr }^{\prime}}{\Longrightarrow}\left(\mathcal{E}^{\prime} ; \mathcal{P}^{\prime} ; \Phi^{\prime} ; \sigma^{\prime}\right)$, there exists a correspondig execution $\left(\mathcal{E}_{0} ; C\left[P_{1}[0] \mid P_{2}[0]\right] ; \Phi ; \emptyset\right) \stackrel{\operatorname{tr}^{\prime \prime}}{\Longrightarrow}\left(\mathcal{E}^{\prime \prime} ; \mathcal{P}^{\prime \prime} ; \Phi^{\prime \prime} ; \sigma^{\prime \prime}\right)$ such that $\sigma^{\prime \prime}$ and $\sigma^{\prime}$ coincide on $\operatorname{dom}\left(\sigma^{\prime \prime}\right)$, and in particular on the values assigned to $x_{1}[\ldots]$ and $x_{2}[\ldots]$. This allows us to deduce 
that $\operatorname{root}\left(x \sigma^{\prime \prime}\right) \notin\{\mathrm{pk}, \mathrm{vk},\langle\rangle\}$, and thus $D^{\mathrm{vseq}}$ satisfies the abstractability property.

The next lemma will allow us to conclude that we obtain traces compatible with $\left(\rho_{\alpha}, \rho_{\beta}\right)$.

Lemma 75: Assume that $D_{n}^{\mathrm{vseq}}$ does not reveal the value of its assignment variables w.r.t. $\left(\rho_{\alpha}, \rho_{\beta}\right)$ and satisfies the abstractability property. We have that for all $D_{n}^{\text {vseq }} \stackrel{\text { tr }}{\Rightarrow}(\mathcal{E} ; \mathcal{P} ; \Phi ; \sigma)$, for all $\gamma \in\{\alpha, \beta\}$, for all $z \in \operatorname{dom}\left(\rho_{\gamma}\right)$, we have that either $\operatorname{tagroot}(z \sigma \downarrow)=\perp$ or tagroot $(z \sigma \downarrow) \notin \gamma \cup\{0\}$.

Proof: Since $D_{n}^{\text {vseq }} \stackrel{\text { tr }}{\Rightarrow}(\mathcal{E} ; \mathcal{P} ; \Phi ; \sigma)$, we know that $(\mathcal{E} ; \mathcal{P} ; \Phi ; \sigma)$ is a derived well-tagged extended process w.r.t. $\prec$ and $\mathrm{col}$, for some $\prec$ and col. Moreover, by construction of $D_{n}^{\text {vseq }}$, we also know that $\operatorname{dom}\left(\rho_{\alpha}\right)=\emptyset$. We prove the result by induction of the $\operatorname{dom}\left(\rho_{\beta}\right)$ with the order $\prec$.

Base case $z \prec z^{\prime}$ for all assignment variables $z^{\prime}$ different from $z$ : Assume that $\operatorname{tag} \operatorname{root}(z \sigma \downarrow) \neq \perp$ and $\operatorname{tagroot}(z \sigma \downarrow) \in$ $\beta \cup\{0\}$. We now show that $z \sigma \downarrow \in F_{c} t_{\alpha}(z \sigma)$. Since $\operatorname{tag} \operatorname{root}(z \sigma \downarrow) \in \beta \cup\{0\}$, we have that if $\operatorname{root}(z \sigma \downarrow) \notin$ $\{\mathrm{vk}, \mathrm{pk},\langle\rangle\}$ then $z \sigma \in F_{c} t_{\alpha}(z \sigma)$. Thus it remains to show that $\operatorname{root}(z \sigma \downarrow) \notin\{\mathrm{vk}, \mathrm{pk},\langle\rangle\}$. But $D_{n}^{\mathrm{vseq}}$ satisfies the abstractability property hence we deduce that $\operatorname{root}(z \sigma \downarrow) \notin\{\mathrm{vk}, \mathrm{pk},\langle\rangle\}$.

Since $z \sigma \downarrow \in F c t_{\alpha}(z \sigma)$, we can apply Lemma 49 and so we deduce that:

1) either there exists $M$ such that $f v(M) \subseteq \operatorname{dom}(\Phi) \cap$ $\left\{z^{\prime} \mid z^{\prime} \prec z\right\}, f n(M) \cap \mathcal{E}=\emptyset$ and $z \sigma \downarrow \in F c t_{\gamma}(M \Phi \downarrow)$

2) otherwise there exists $j$ such that $z_{j}^{\beta} \prec z$ and $z_{j}^{\beta} \sigma \downarrow=$ $z \sigma \downarrow$

The second case is trivially impossible since $\operatorname{dom}\left(\rho_{\alpha}\right)=\emptyset$ and so $z_{j}^{\beta}$ does not exists. We focus on the first case: We know that $z \sigma \downarrow \in \operatorname{Fct}_{\gamma}(M \Phi \downarrow)$. Since $z \sigma \downarrow$ is not deducible in new $\mathcal{E} . \Phi$, then $z \sigma \downarrow \notin F c t_{\langle\rangle}(M \Phi \downarrow)$. Moreover, we know that for all assignment variables $z^{\prime}$ different from $z, z \prec z^{\prime}$. Thus we can apply Lemma 51 and obtain that there exists $M^{\prime \prime}$ such that $f v(M) \subseteq \operatorname{dom}(\Phi), f n(M) \cap \mathcal{E}=\emptyset$ and $z \sigma \downarrow \in$

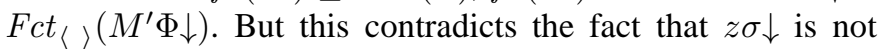
deducible in new $\mathcal{E} . \Phi$.

Since we always reach a contradiction, we can conclude that tagroot $(z \sigma \downarrow)=\perp$ or $\operatorname{tagroot}(z \sigma \downarrow) \notin \beta \cup\{0\}$.

Inductive case: Assume once again that $\operatorname{tagroot}(z \sigma \downarrow) \neq \perp$ and tagroot $(z \sigma \downarrow) \in \beta \cup\{0\}$. As in the previous case, we can show that $z \sigma \downarrow \in F c t_{\alpha}(z \sigma)$ and so we can apply Lemma 49 to obtain:

1) either there exists $M$ such that $f v(M) \subseteq \operatorname{dom}(\Phi) \cap$ $\left\{z^{\prime} \mid z^{\prime} \prec z\right\}, f n(M) \cap \mathcal{E}=\emptyset$ and $z \sigma \downarrow \in F_{c} t_{\gamma}(M \Phi \downarrow)$

2) otherwise there exists $j$ such that $z_{j}^{\beta} \prec z$ and $z_{j}^{\beta} \sigma \downarrow=$ $z \sigma \downarrow$

Once again the first case is trivially impossible since $\operatorname{dom}\left(\rho_{\alpha}\right)=\emptyset$. Thus it remain to focus on the second case. As in the previous, we can deduce that $z \sigma \downarrow \notin F c t_{\langle\rangle}(M \Phi \downarrow)$. Moreover, by our inductive hypothesis, we know that for all assignment variable $z^{\prime} \prec z, \operatorname{tagroot}\left(z^{\prime} \sigma \downarrow\right)=\perp$ or $\operatorname{tag} \operatorname{root}\left(z^{\prime} \sigma \downarrow\right) \notin \beta \cup\{0\}$. Thus, we can deduce that $z^{\prime} \sigma \downarrow \neq$ $z \sigma \downarrow$. Thanks to this, we can apply Lemma 51 and obtain that there exists $M^{\prime \prime}$ such that $f v(M) \subseteq \operatorname{dom}(\Phi), f n(M) \cap \mathcal{E}=\emptyset$ and $z \sigma \downarrow \in F c t_{\langle\rangle}\left(M^{\prime} \Phi \downarrow\right)$. But this contradicts the fact that $z \sigma \downarrow$ is not deducible in new $\mathcal{E}$. $\Phi$.

Since we always reach a contradiction, we can conclude that $\operatorname{tagroot}(z \sigma \downarrow)=\perp$ or tagroot $(z \sigma \downarrow) \notin \beta \cup\{0\}$.

We now establish that when the processes are a good key exchanged protocol, all possible executions are actually compatible w.r.t. $\left(\rho_{\alpha}, \rho_{\beta}\right)$.

Lemma 76: Let $\left(\rho_{\alpha}, \rho_{\beta}\right)$ be the two abstraction functions as defined in Section E-A. If $\left(\mathcal{E}_{0} ; C\left[P_{1}[0] \mid P_{2}[0]\right] ; \Phi ; \emptyset\right)$ satisfies the abstractability property and $P_{1} / P_{2} / \Phi$ is a good keyexchanged protocol under $\mathcal{E}_{0}$ and $C$ then for any $P$ such that:

- $\mathrm{fst}\left(D_{n}^{\mathrm{vseq}}\right) \stackrel{\text { tr }}{\Rightarrow} P$ (resp. $\operatorname{snd}\left(D_{n}^{\mathrm{vseq}}\right) \stackrel{\text { tr }}{\Rightarrow} P$ ), we have that $P$ is compatible w.r.t. $\left(\rho_{\alpha}, \rho_{\beta}\right)$.

- $\operatorname{fst}\left(S_{n}^{\mathrm{v}}\right) \stackrel{\operatorname{tr}}{\Rightarrow} P\left(\right.$ resp. $\left.\operatorname{snd}\left(S_{n}^{\mathrm{v}}\right) \stackrel{\text { tr }}{\Rightarrow} P\right)$, we have that $P$ is compatible w.r.t. $\left(\rho_{\alpha}, \rho_{\beta}\right)$.

Proof: Let $P$ be a process such that $\mathrm{fst}\left(D_{n}^{\mathrm{vseq}}\right) \stackrel{\text { tr }}{\Rightarrow}(\mathcal{E} ; \mathcal{P} ; \Phi$; $\sigma)$. Let $x, y \in \operatorname{dom}(\sigma) \cap \operatorname{dom}\left(\rho_{\beta}\right)$ and assume that $x \sigma={ }_{\mathrm{E}}$ $y \sigma$. Let us denote $x=x_{i}\left[i_{1}, \ldots, i_{m}\right]$ and $y=x_{j}\left[j_{1}, \ldots, j_{m}\right]$ where $j_{k}, i_{k} \in\{1, \ldots, n\}, k \in\{1, \ldots, m\}$ and $i, j \in\{1,2\}$.

By hypothesis, $P_{1} / P_{2} / \Phi$ is a good key-exchanged protocol under $\mathcal{E}_{0}$ and $C$. Hence thanks to Lemma 71, $x \sigma={ }_{\mathrm{E}}$ $y \sigma$ implies that $i_{k}=j_{k}$ for all $k \in\{1 \ldots m\}$. On the other hand, Lemma 71 also indicates that $x_{1}\left[i_{1}, \ldots, i_{m}\right] \sigma=$ $x_{2}\left[i_{1}, \ldots, i_{m}\right] \sigma$, for all $i_{1}, \ldots, i_{m}$.

Since by definition of $\rho_{\beta}, x_{1}\left[i_{1}, \ldots, i_{m}\right] \rho_{\beta}=$ $x_{2}\left[i_{1}, \ldots, i_{m}\right] \rho_{\beta}=k\left[i_{1}, \ldots, i_{m}\right]$, we can deduce that $x \sigma=y \sigma$ if and only if $x \rho_{\beta}=y \rho_{\beta}$. At last, relying on Lemma 75 , we can conclude that $(\mathcal{E} ; \mathcal{P} ; \Phi ; \sigma)$ is compatible with $\left(\rho_{\alpha}, \rho_{\beta}\right)$.

We now prove the property for $S_{n}^{\vee}$ : Let $\mathrm{fst}\left(S_{n}^{\mathrm{v}}\right) \stackrel{\text { tr }}{\Rightarrow}(\mathcal{E} ; \mathcal{P} ; \Phi ; \sigma)$. We prove the result by induction on the size of tr. Consider a transition $(\mathcal{E} ; \mathcal{P} ; \Phi ; \sigma) \stackrel{\ell}{\rightarrow} A$. By inductive hypothesis, we know that $(\mathcal{E} ; \mathcal{P} ; \Phi ; \sigma)$ is compatible with $\left(\rho_{\alpha}, \rho_{\beta}\right)$. But, the only transition that could render $A$ not compatible is the internal transition (ASSGN). Hence assume that $\mathcal{P}=\left\{[x:=t]^{i} . P\right\} \uplus \mathcal{Q}$ where $i \in \gamma$ and $\ell=\tau$.

Since $(\mathcal{E} ; \mathcal{P} ; \Phi ; \sigma)$ is compatible, then by Theorem 31 and in particular Proposition 56, we deduce that $\mathrm{fst}\left(D_{n}^{\mathrm{vseq}}\right) \stackrel{\text { tr }}{\Rightarrow}\left(\mathcal{E}^{\prime} ; \mathcal{P}^{\prime} ; \Phi^{\prime} ; \sigma^{\prime}\right) \quad$ where $\quad \delta(\sigma \downarrow)=\sigma^{\prime} \downarrow$ and $\delta(\mathcal{P})=\mathcal{P}^{\prime}$. It implies that $\mathcal{P}^{\prime}=\left\{\left[x:=\delta_{\gamma}(t)\right]^{i} . \delta(P)\right\} \uplus \delta(\mathcal{Q})$. Thus, by Lemma 38, we have that $\delta_{\gamma}(t \sigma \downarrow)=\delta_{\gamma}(t) \sigma^{\prime} \downarrow$.

On the other hand, if $A$ is not compatible, it means that there exists $y \in \operatorname{dom}(\sigma)$ such that $t \sigma \downarrow=y \sigma \downarrow$ is not equivalent to $x \rho_{\beta}=y \rho_{\beta}$. But $\delta(\sigma \downarrow)=\sigma^{\prime} \downarrow$ and $\delta_{\gamma}(t \sigma \downarrow)=\delta_{\gamma}(t) \sigma^{\prime} \downarrow$. Hence $t \sigma \downarrow=y \sigma \downarrow$ is equivalent to $\delta_{\gamma}(t) \sigma^{\prime}=y \sigma^{\prime}$, and so we can deduce that $\delta_{\gamma}(t) \sigma^{\prime}=y \sigma^{\prime}$ is not equivalent to $x \rho_{\beta}=y \rho_{\beta}$. However, $\left(\mathcal{E}^{\prime} ; \mathcal{P}^{\prime} ; \Phi^{\prime} ; \sigma^{\prime}\right)$ can also apply the internal transition (ASSGN) on $[x:=\delta(t)]^{i}$ and so we obtain $\left(\mathcal{E}^{\prime} ; \mathcal{P}^{\prime} ; \Phi^{\prime} ; \sigma^{\prime}\right) \stackrel{\tau}{\rightarrow} A^{\prime}$ with $A^{\prime}$ not compatible with $\left(\rho_{\alpha}, \rho_{\beta}\right)$. This is in contradiction with our result on $D_{n}^{\mathrm{vseq}}$.

\section{Composing diff-equivalence}

We are now able to prove our composition results. 
Theorem 17: Let $C$ be a composition context and $\mathcal{E}_{0}$ be a finite set of names of base type. Let $P_{1}\left[\right.$ ] (resp. $P_{2}\left[\_\right]$) be a plain biprocess without replication and with an hole in the scope of an assignment of the form $\left[x_{1}:=t_{1}\right]$ (resp. $\left[x_{2}:=t_{2}\right]$ ). Let $Q_{1}$ (resp. $Q_{2}$ ) be a plain biprocess such that $f v\left(Q_{1}\right) \subseteq\left\{x_{1}\right\}$ (resp. $f v\left(Q_{2}\right) \subseteq\left\{x_{2}\right\}$ ), and $\Phi$ and $\Psi$ be two frames. Let $P=P_{1}[0] \mid P_{2}[0]$ and $Q=$ new $k .\left[x_{1}:=\right.$ $k] .\left[x_{2}:=k\right] .\left(Q_{1} \mid Q_{2}\right)$ for some fresh name $k$, and assume that:

1) $P / \Phi$ and $Q / \Psi$ are composable under $\mathcal{E}_{0}$ and $C$;

2) $\left(\mathcal{E}_{0} ; C[Q] ; \Psi\right)$ does not reveal $k, \operatorname{pk}(k), \operatorname{vk}(k)$;

3) $\left(\mathcal{E}_{0} ; C[P] ; \Phi\right)$ satisfies the abstractability property; and

4) $P_{1} / P_{2} / \Phi$ is a good key-exchange protocol under $\mathcal{E}_{0}$ and $C$.

If $\left(\mathcal{E}_{0} ; C[P] ; \Phi\right)$ and $\left(\mathcal{E}_{0} ; C[Q] ; \Psi\right)$ satisfy diff-equivalence, then $\left(\mathcal{E}_{0} ; C\left[P_{1}\left[Q_{1}\right] \mid P_{2}\left[Q_{2}\right]\right] ; \Phi \uplus \Psi\right)$ satisfies diff-equivalence.

Proof: Let $S=\left(\mathcal{E}_{0} ; C\left[P_{1}\left[Q_{1}\right] \mid P_{2}\left[Q_{2}\right]\right] ; \Phi \uplus \Psi ; \emptyset\right)$. Thanks to Lemma 59, we know that $S$ is in diff-equivalence if, and only if, $S_{n}$ is in diff-equivalence for all $n \in \mathbb{N}$.

By hypothesis, we know that:

- $\left(\mathcal{E}_{0} ; C\left[P_{1}[0] \mid P_{2}[0]\right] ; \Phi ; \emptyset\right)$, and

- $\left(\mathcal{E}_{0} ; C\left[\right.\right.$ new $\left.\left.k .\left(Q_{1}\left\{k / x_{1}\right\} \mid Q_{2}\left\{k / x_{2}\right\}\right)\right] ; \Psi ; \emptyset\right)$

are both in diff-equivalence and $P_{1}, P_{2}, Q_{1}, Q_{2}$ are tagged. Hence, since diff-equivalence is preserved by disjoint parallel composition, we deduce that $D^{\text {par }}$ is in diff-equivalence, and thus, thanks to Lemma 59, we obtain that $D_{n}^{\text {par }}$ is in diffequivalence for all $n \in \mathbb{N}$. Applying Lemma 68, we deduce that $D_{n}^{\text {seq }}$ is also in diff-equivalence. Note that diff-equivalence still holds on the biprocess $D_{n}^{\text {vseq }}$ obtained from $D_{n}^{\text {seq }}$ by adding some assignment variables to "explicit the sharing".

Given $n \in \mathbb{N}$, in order to conclude, we have to show that $S_{n}^{\vee}$ obtained from $S_{n}$ by adding some assignments variables to explicit the sharing satisfies diff-equivalence. We form two new biprocesses $S D_{L}$ and $S D_{R}$ as follows:

- $\operatorname{fst}\left(S D_{L}\right)=\mathrm{fst}\left(S_{n}^{\mathrm{v}}\right)$ and $\operatorname{snd}\left(S D_{L}\right)=\mathrm{fst}\left(D_{n}^{\mathrm{vseq}}\right)$;

- $\operatorname{fst}\left(S D_{R}\right)=\operatorname{snd}\left(S_{n}^{\mathrm{v}}\right)$ and $\operatorname{snd}\left(S D_{R}\right)=\operatorname{snd}\left(D_{n}^{\mathrm{vseq}}\right)$;

We will apply Theorem 31 on biprocesses $S D_{L}$ and $S D_{R}$ to establish the strong relationship between the two components of each biprocess, and together with the fact $D_{n}^{\text {vseq }}$ satisfies diff-equivalence, this will allow us to conclude that $S_{n}^{\vee}$ satisfies diff-equivalence too.

Considering the two abstraction functions $\left(\rho_{\alpha}, \rho_{\beta}\right)$ as defined in Section E-A, in order to apply Theorem 31 on $S D_{L}$ (resp. $\left.S D_{R}\right)$, it remains to show that $\operatorname{fst}\left(D_{n}^{\mathrm{vseq}}\right)$ and $\operatorname{snd}\left(D_{n}^{\mathrm{vseq}}\right)$ do not reveal the value of their assignment variables w.r.t. $\left(\rho_{\alpha}, \rho_{\beta}\right)$. This is actually achieved by application of Lemma 70 with the facts that

- $\left(\mathcal{E}_{0} ; C\left[\right.\right.$ new $\left.\left.k .\left(Q_{1}\left\{k / x_{1}\right\} \quad Q_{2}\left\{k / x_{2}\right\}\right)\right] ; \Psi ; \emptyset\right)$ and $\left(\mathcal{E}_{0} ; C\left[P_{1}[0] \quad \mid \quad P_{2}[0]\right] ; \Phi ; \emptyset\right)$ do not reveal key in $\left\{n, \operatorname{pk}(n), \operatorname{vk}(n) \mid n \in f n\left(P_{1}, P_{2}\right) \cap f n\left(Q_{1}, Q_{2}\right) \cap b n(C)\right\}$, and

- $\left(\mathcal{E}_{0} ; C\left[\right.\right.$ new $\left.\left.k .\left(Q_{1}\left\{{ }^{k} / x_{1}\right\} \mid Q_{2}\left\{{ }^{k} / x_{2}\right\}\right)\right] ; \Psi ; \emptyset\right)$ do not reveal $k, \operatorname{pk}(k), \mathrm{vk}(k)$, and
- $P_{1} / P_{2} / \Phi$ is a good key-exchange protocol under $\mathcal{E}_{0}$ and $C$, that is $\left(\mathcal{E}_{0} ; P_{\text {good }} ; \Phi\right)$ does not reveal bad where $P_{\text {good }}$ is defined as follows:

$$
\begin{aligned}
& P_{\text {good }}=\text { new } b a d, d .( \\
& C\left[\text { new } i d .\left(P_{1}\left[\operatorname{out}\left(d,\left\langle x_{1}, i d\right\rangle\right)\right] \mid P_{2}\left[\operatorname{out}\left(d,\left\langle x_{2}, i d\right\rangle\right)\right]\right)\right] \\
& \mid \operatorname{in}(d, x) . \operatorname{in}(c, z) . \\
& \quad \text { if } z \in\left\{\operatorname{proj}_{1}(x), \operatorname{pk}\left(\operatorname{proj}_{1}(x)\right), \operatorname{vk}\left(\operatorname{proj}_{1}(x)\right)\right\} \\
& \quad \text { then out }(c, b a d))
\end{aligned}
$$

Now, let $B_{S}$ be a biprocess such that

$$
S_{n}^{\vee} \stackrel{\text { tr }_{\mathrm{bi}}}{\Rightarrow} B_{S} \stackrel{\text { def }}{=}\left(\mathcal{E}_{S} ; \mathcal{P}_{S} ; \Phi_{S} ; \sigma_{S}\right)
$$

for some tr. By definition of diff-equivalene, we have to show that:

1) new $\mathcal{E}_{S}$.fst $\left(\Phi_{S}\right) \sim$ new $\mathcal{E}_{S}$.snd $\left(\Phi_{S}\right)$;

2) if $\operatorname{fst}\left(B_{S}\right) \stackrel{\ell}{\rightarrow} A_{L}$ then there exists $B^{\prime}$ such that $B_{S} \stackrel{\ell}{\rightarrow}_{\mathrm{bi}} B^{\prime}$ and $\mathrm{fst}\left(B^{\prime}\right)=A_{L}$ (and similarly for snd).

We have $\mathrm{fst}\left(S_{n}^{\mathrm{v}}\right) \stackrel{\text { tr }}{\Rightarrow} \mathrm{fst}\left(B_{S}\right)$ as well as $\operatorname{snd}\left(S_{n}^{\mathrm{v}}\right) \stackrel{\text { tr }}{\Rightarrow} \operatorname{snd}\left(B_{S}\right)$. By Lemma 76 , we obtain that $\operatorname{fst}\left(B_{S}\right)$ as well as $\operatorname{snd}\left(B_{S}\right)$ is compatible with $\left(\rho_{\alpha}, \rho_{\beta}\right)$. Hence, relying on Theorem 31 (first item), we deduce that there exist biprocesses $S D_{L}^{\prime}$ and $S D_{R}^{\prime}$ such that:

- $S D_{L} \stackrel{\text { tr }}{\Rightarrow}$ bi $S D_{L}^{\prime}$, fst $\left(S D_{L}^{\prime}\right)=\mathrm{fst}\left(B_{S}\right)$, and static equivalence holds between the two frames issued from the biprocess $S D_{L}^{\prime}$;

- $S D_{R} \stackrel{\text { tr }}{\Rightarrow}{ }_{\mathrm{bi}} S D_{R}^{\prime}$, fst $\left(S D_{R}^{\prime}\right)=\operatorname{snd}\left(B_{S}\right)$, and static equivalence holds between the two frames issued from the biprocess $S D_{R}^{\prime}$.

Since, we know that $D_{n}^{\text {vseq }}$ satisfies diff-equivalence, we have that $D_{n}^{\text {vseq }} \stackrel{\text { tr }}{\Rightarrow}{ }_{\mathrm{bi}} D_{n}^{\text {vvseq }}$ with $\operatorname{fst}\left(D_{n}^{\text {/vseq }}\right)=\operatorname{snd}\left(S D_{L}^{\prime}\right)$ and $\operatorname{snd}\left(D_{n}^{\prime \text { vseq }}\right)=\operatorname{snd}\left(S D_{R}^{\prime}\right)$. Then, by transitivity of static equivalence, we deduce that

$$
\text { new } \mathcal{E}_{S} \text {.fst }\left(\Phi_{S}\right) \sim \text { new } \mathcal{E}_{S} \cdot \operatorname{snd}\left(\Phi_{S}\right) \text {. }
$$

Now, assume that $\mathrm{fst}\left(B_{S}\right) \stackrel{\ell}{\rightarrow} A_{L}$. In such a case, we have that $\mathrm{fst}\left(S_{n}\right) \stackrel{\text { tr }}{\Rightarrow} \mathrm{fst}\left(B_{S}\right) \stackrel{\ell}{\rightarrow} A_{L}$. By Lemma 76 , we obtain that $A_{L}$ is compatible with $\left(\rho_{\alpha}, \rho_{\beta}\right)$, and relying on Theorem 31 (first item), we deduce that there exists a biprocess $S D_{L}^{\prime \prime}$ such that: $S D_{L} \stackrel{\text { tr }^{\Rightarrow}}{\text { bi }} \stackrel{\ell}{\rightarrow}_{\text {bi }} S D_{L}^{\prime \prime}$ with fst $\left(S D_{L}^{\prime \prime}\right)=A_{L}$. Since $D_{n}^{\text {vseq }}$ satisfies diff-equivalence, we have that $D_{n}^{\text {vseq }} \stackrel{\text { tr }}{\Rightarrow}$ bi $\stackrel{\ell}{\rightarrow}_{\text {bi }} D_{n}^{\prime \prime \text { vseq }}$ for some biprocess $D_{n}^{\prime \prime \text { vseq }}$ with $\operatorname{fst}\left(D_{n}^{\prime \prime \text { vseq }}\right)=\operatorname{snd}\left(S D_{L}^{\prime \prime}\right)$. Now, applying Theorem 31 (second item) on biprocess $S D_{R}$, we deduce that $S D_{R} \stackrel{\operatorname{tr}}{\Rightarrow}_{\text {bi }}-\stackrel{\ell}{\rightarrow}_{\text {bi }} S D_{R}^{\prime \prime}$ with $\operatorname{snd}\left(S D_{R}^{\prime \prime}\right)=$ $\operatorname{snd}\left(D_{n}^{\prime \prime v s e q}\right)$. This allows us to ensure the existence of the biprocess $B^{\prime}$ required to show diff-equivalence of $S_{n}^{\vee}$. We will have $\mathrm{fst}\left(B^{\prime}\right)=\mathrm{fst}\left(S D_{L}^{\prime \prime}\right)=A_{L}$ and $\operatorname{snd}\left(B^{\prime}\right)=\mathrm{fst}\left(S D_{R}^{\prime \prime}\right)$.

\section{E. Composing reachability}

Corollary 18: Under the same hypotheses as Theorem 17 with processes instead of biprocesses, and considering a name $s$ that occurs in $C$. If $\left(\mathcal{E}_{0} ; C[P] ; \Phi\right)$ and $\left(\mathcal{E}_{0} ; C[Q] ; \Psi\right)$ do not reveal $s$ then $\left(\mathcal{E}_{0} ; C\left[P_{1}\left[Q_{1}\right] \mid P_{2}\left[Q_{2}\right]\right] ; \Phi \uplus \Psi\right)$ does not reveal $s$. 
Proof: Let $S=\left(\mathcal{E}_{0} ; C\left[P_{1}\left[Q_{1}\right] \mid P_{2}\left[Q_{2}\right]\right] ; \Phi \uplus \Psi ; \emptyset\right)$. By hypothesis, we know that:

- $\left(\mathcal{E}_{0} ; C\left[P_{1}[0] \mid P_{2}[0]\right] ; \Phi ; \emptyset\right)$, and

- $\left(\mathcal{E}_{0} ; C\left[\right.\right.$ new $\left.\left.k .\left(Q_{1}\left\{k / x_{1}\right\} \mid Q_{2}\left\{k / x_{2}\right\}\right)\right] ; \Psi ; \emptyset\right)$

does not reveal $s$. Since, secrecy is preserved by disjoint composition, and the transformations introduced at the beginning of the section (e.g. unfolding, adding assignment variables, ...), we easily deduce that $D_{n}^{\text {vseq }}$ do not reveal $s$.

We show the result by contradiction. Assume that $S_{n}^{\vee}$ reveals the secrecy $s$. We consider a trace witnessing this fact, i.e. a process $S_{n}^{\prime v}$ such that

$$
S_{n}^{\vee \stackrel{\text { tr }}{\Rightarrow}} S_{n}^{\prime \vee} \stackrel{\text { def }}{=}\left(\mathcal{E}_{S} ; \mathcal{P}_{S} ; \Phi_{S} ; \sigma_{S}\right)
$$

and for which new $\mathcal{E}_{S}$. $\Phi_{S} \vdash s$.

We form a biprocess $S D$ by grouping together $S_{n}^{\vee}$ and $D_{n}^{\text {vseq }}$ in order to apply Theorem 31 .

In order to apply Theorem 31, we first must prove that $D_{n}^{\text {vseq }}$ does not reveal the value of its assignment variables w.r.t. $\left(\rho_{\alpha}, \rho_{\beta}\right)$ as defined in Section E-A. This is achieved by application of Lemma 70 with the facts that

- $\left(\mathcal{E}_{0} ; C\left[\right.\right.$ new $\left.\left.\left.k .\left(Q_{1}\left\{{ }^{k} / x_{1}\right\} \quad \mid \quad Q_{2}{ }^{k} / x_{2}\right\}\right)\right] ; \Psi ; \emptyset\right)$ and $\left(\mathcal{E}_{0} ; C\left[P_{1}[0] \quad \mid \quad P_{2}[0]\right] ; \Phi ; \emptyset\right)$ do not reveal key in $\left\{n, \operatorname{pk}(n), \operatorname{vk}(n) \mid n \in f n\left(P_{1}, P_{2}\right) \cap f n\left(Q_{1}, Q_{2}\right) \cap b n(C)\right\}$, and

- $\left(\mathcal{E}_{0} ; C\left[\right.\right.$ new $\left.\left.k \cdot\left(Q_{1}\left\{{ }^{k} / x_{1}\right\} \mid Q_{2}\left\{k / x_{2}\right\}\right)\right] ; \Psi ; \emptyset\right)$ do not reveal $k, \operatorname{pk}(k), \operatorname{vk}(k)$, and

- $P_{1} / P_{2} / \Phi$ is a good key-exchange protocol under $\mathcal{E}_{0}$ and $C$, that is $\left(\mathcal{E}_{0} ; P_{\text {good }} ; \Phi\right)$ does not reveal bad where $P_{\text {good }}$ is defined as follows:

$$
\begin{aligned}
& P_{\text {good }}=\text { new bad, } d .( \\
& C\left[\text { new } i d .\left(P_{1}\left[\operatorname{out}\left(d,\left\langle x_{1}, i d\right\rangle\right)\right] \mid P_{2}\left[\operatorname{out}\left(d,\left\langle x_{2}, i d\right\rangle\right)\right]\right)\right] \\
& \mid \text { in }(d, x) . \operatorname{in}(c, z) . \\
& \quad \text { if } z \in\left\{\operatorname{proj}_{1}(x), \operatorname{pk}\left(\operatorname{proj}_{1}(x)\right), \operatorname{vk}\left(\operatorname{proj}_{1}(x)\right)\right\} \\
& \quad \text { then out }(c, \text { bad }))
\end{aligned}
$$

As done previously, relying on Lemma 76, we may assume that the trace under study is compatible. Applying Theorem 31, we deduce that there exists a biprocess $S D^{\prime}$ such that $S D \stackrel{\mathrm{tr}}{\Rightarrow}$ bi $S D^{\prime}$ with fst $\left(S D^{\prime}\right)=S_{n}^{\prime \mathrm{v}}$, and static equivalence holds between the two frames issued from the biprocess $S D^{\prime}$. Moreover, if we denote by $\Phi_{S}$ and $\Phi_{D}$ the respective frame of $\operatorname{fst}\left(S D^{\prime}\right)$ and $\operatorname{snd}\left(S D^{\prime}\right)$, we ensure that $\delta\left(\Phi_{S} \downarrow\right)=\Phi_{D} \downarrow$ (see Proposition 56).

Therefore, since $D_{n}^{\text {vseq }}$ does not reveal the secret $s$, and we already proved that $D_{n}^{\text {vseq }}$ does not reveal his assignment variables, then by Lemma 53, we can deduce that $S_{n}^{\vee}$ does not reveal $s$, and so $S$ does not reveal $s$ either.

\section{APPENDIX F}

\section{MOBILE PHONES PROTOCOLS DETAILED}

In this section we further detail the case study on $3 \mathrm{G}$ telecommunication protocols.

\section{A. The Authentication and Key Agreement (AKA) protocol}

This protocol achieves mutual authentication between a Mobile Station (MS) and the network, and allows them to establish shared session keys to be used to secure subsequent communications.

The $A K A$ protocol consists in the exchange of two messages: the authentication request and the authentication response. The authentication request contains the authentication challenge $R A N D$ and the authentication token $A U T N$ to the mobile station. AUTN contains a MAC of the concatenation of the random number with a sequence number $S Q N_{N}$ generated by the network using an individual counter for each subscriber. A new sequence number is generated either by increment of the counter or through time based algorithms as defined in [2]. The sequence number $S Q N_{N}$ allows the mobile station to verify the freshness of the authentication request to defend against replay attacks.

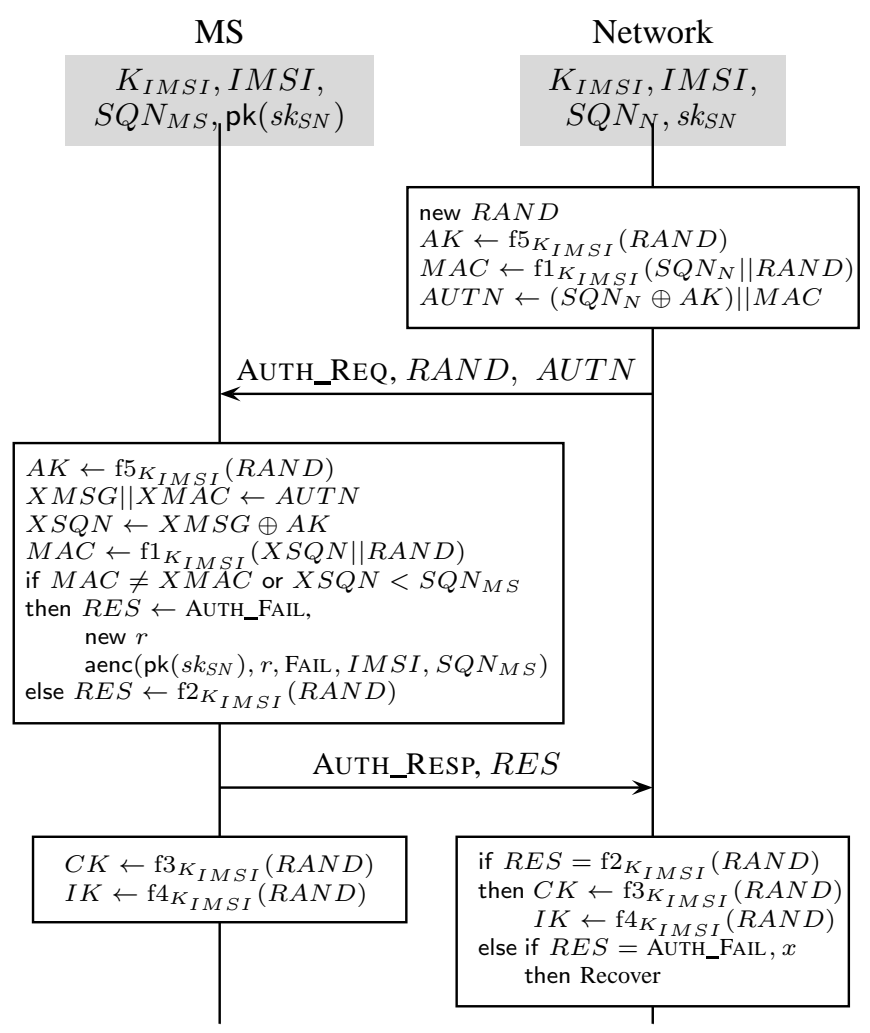

The MS receives the authentication request, retrieves the sequence number $S Q N_{N}$ and then verifies the MAC (condition $M A C=X M A C)$. This step ensures that the MAC was generated by the network using the shared key $K_{I M S I}$, and thus that the authentication request was intended for the mobile station with identity IMSI. The mobile station stores the greatest sequence number used for authentication, so far $S Q N_{M S}$. This value is used to check the freshness of the authentication request (condition $X S Q N<S Q N_{M S}$ ) to avoid replay attacks.

The mobile station computes the ciphering key $C K$, the integrity key $I K$ and the authentication response $R E S$ and sends this response to the network. The network authenticates 
the mobile station by verifying whether the received response is equal to the expected one $\left(R E S=\mathrm{f} 2_{K}(R A N D)\right)$. The authentication procedure can fail on the MS side either because the MAC verification failed, or because the received sequence number $X S Q N$, is not in the correct range with respect to the sequence number $S Q N_{M S}$ stored in the mobile station. In the former case, the mobile station sends an authentication failure message indicating MAC failure (MAC_FAIL) as the failure cause. In the latter case, the authentication failure message indicates synchronisation failure (SYNC_FAIL) as the failure cause. When a MAC failure occurs the network may initiate the identification procedure. When a synchronisation failure occurs the network performs re-synchronisation.

\section{B. The Send SMS (sSMS) procedure}

It allows a MS to send an SMS to another MS through the Network.

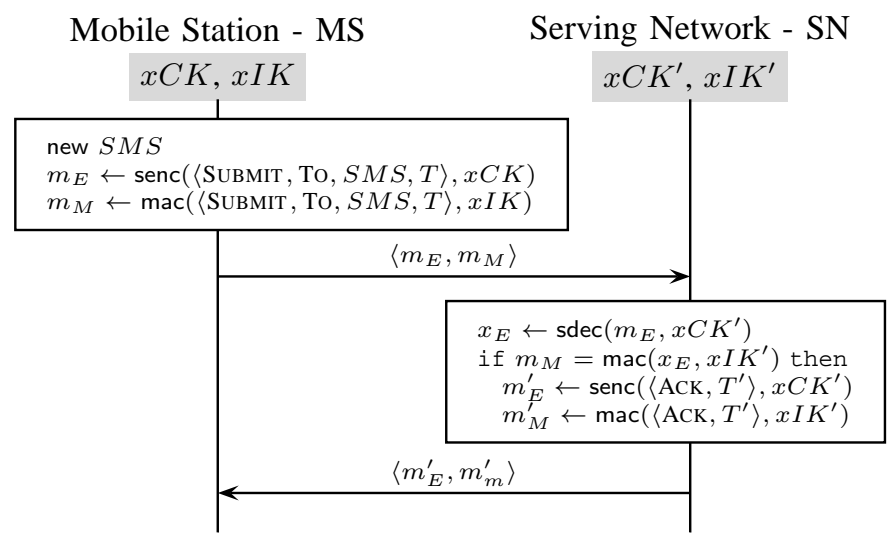

The confidentiality of the sent SMS relies on the session key $C K$. And its integrity on the session key $I K$. These two keys are established through the execution of the $A K A$ protocol between the MS and the network. The key $C K$ is used to encrypt the two messages exchanged during the execution of the $s S M S$ procedure. The key $I K$ is used to compute a MAC digest of these two messages of the content of the sent SMS.

It is always the MS that initiates the $s S M S$ procedure. It does so by encrypting the content of the SMS it wants to submit, together with the number of the destination MS and a timestamp $T$, with the session key $C K$ previously established. The message also contains a constant SUBMIT. To this it appends the MAC digest of the encrypted message computed using the integrity key $I K$. The Network checks that the MAC digest corresponds to the encrypted message using the integrity key $I K$, and if it does, it acknowledges the receipt of the message with a message that includes a constant ACK and a timestamp $T^{\prime}$ encrypted with $C K$.

\section{Discussion on ProVerif models of the AKA and sSMS protocols}

To enable the use of ProVerif to illustrate our results we had to abstract some details of the considered protocols that ProVerif cannot handle. We discuss in this section these abstractions. a) The XOR operation: In the execution of $A K A$, the athentication token $A U T N$ is computed by XORing the current $S Q N_{N}$ with the authentication key $A K$. Since ProVerif cannot handle the XOR operation, we abstract this operation by simply modelling it as symmetric encryption. More precisely, in our model of the protocol, the $S Q N_{N}$ is symmetrically encrypted using the key $A K$.

b) The sequence number $S Q N$ : The security of the $A K A$ protocol relies on the lose synchronisation of the MS and the SN on a counter $S Q N_{N}$. Unfortunately, ProVerif cannot efficiently reason about integers and $\leq$ so we assume that the MS and the SN are "magically" synchronised on this counter. This is modelled by assuming at each session of the $A K A$ protocol a shared name sqn.

c) The integrity key $I K$ : For simplicity reasons we presented our results restricted to the sharing of a single assignment variable. Although this was not fundamentally needed for our results to hold, it does prohibit us from sharing both the $C K$ and the $I K$ keys between the $A K A$ protocol and the $s S M S$. This is why we have omitted in our model the integrity messages and integrity checks. Note that these integrity mechanisms are not necessary for ensuring the properties we were interested in our case study.

d) Reasoning about timestamps: ProVerif cannot handle timestamps. One way to model timestamps would have been to abstract them by nonces. However, timestamps are not secret nor unguessable, so abstracting them by nonces unreasonably restricts the attacker's power. For this reason we do not model timestamps as nonces, but we rather let the attacker input the timestamps' value. Hence the two input messages in $(c$, time $)$ in the ProVerif scripts corresponding to our case study. 